\title{
Personalizing CFTR \\ modulator therapies
}

Gitte Berkers 

Personalizing CFTR modulator therapies

Gitte Berkers 
Personalizing CFTR modulator therapies

PhD thesis, Utrecht University, The Netherlands

(๑) Gitte Berkers, 2020, Utrecht, The Netherlands

ISBN: 978-90-393-7268-5

Cover design and layout: Jan Lücker, KEK dnl I vormgevers bno

Printed by: Proefschriftmaken.nl

Printing of this thesis was financially supported by:

Chiesie Pharmaceuticals B.V., Chipsoft, Corning, DEKAsvitamins.com, Essential Organics ADEK,

Eurocept Homecare, Mediq Romedic, Pentax Medical, Teva Netherlands B.V., Westfalen Medical B.V. 


\title{
PERSONALIZING CFTR MODULATOR THERAPIES
}

\author{
Personaliseren van CFTR modulerende therapieën \\ (met een samenvatting in het Nederlands)
}

\section{Proefschrift}

ter verkrijging van de graad van doctor aan de Universiteit Utrecht

op gezag van de rector magnificus prof. dr. H.R.B.M. Kummeling,

ingevolge het besluit van het college voor promoties in het openbaar te verdedigen op

dinsdag 1 september 2020 des middags te 2.30 uur

door

\section{Gitte Berkers}


Promotoren:

Prof. Dr. C.K. van der Ent

Prof. Dr. J.M. Beekman 


\section{CONTENTS}

Chapter 1 General introduction

\section{Part I - In vivo effect of modulators of CFTR function}

Chapter $2 \quad$ B2-Adrenergic receptor agonists activate CFTR in

intestinal organoids and subjects with cystic fibrosis

Chapter 3 Clinical effects of the three CFTR potentiator treatments curcumin, genistein and ivacaftor in patients with the CFTR-S1251N gating mutation

Chapter 4 Ivacaftor restores FGF19 regulated bile acid homeostasis in cystic fibrosis patients with an S1251N or a G551D gating mutation

Chapter 5 A phase 2 crossover study of lumacaftor/ivacaftor in cystic fibrosis patients with an A455E-CFTR mutation

\section{Part II - Predicting in vivo CFTR function with organoids}

Chapter 6 Forskolin-induced swelling of intestinal organoids correlates with disease severity in adults with cystic fibrosis and homozygous F508del mutations

Chapter 7 Characterizing responses to CFTR-modulating drugs using rectal organoids derived from subjects with cystic fibrosis

Chapter 8 Rectal organoids enable personalized treatment of cystic fibrosis

Chapter 9 General discussion

Chapter 10 Summary in Dutch - Nederlandse samenvatting

Addenda Abbreviations

Contributing authors

Acknowledgements - Dankwoord

About the author 


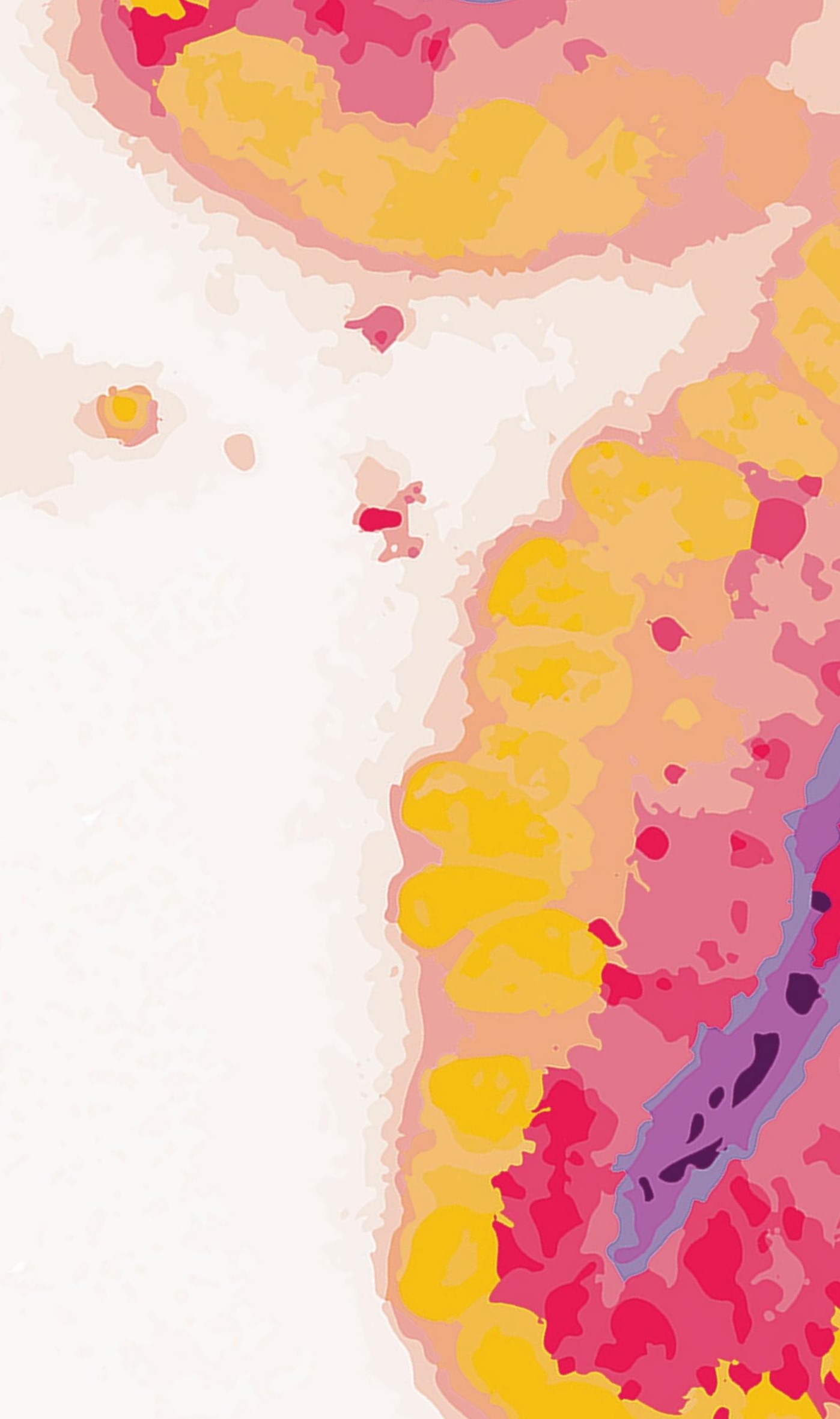




\section{CHAPTER 1}

GENERAL INTRODUCTION 
Recent advances in stem cell culture approaches have led to the generation of patientspecific living biobanks for many diseases. These resources can play an important role in preclinical drug development, but data on the clinical predictive capacity of these models is lacking. This stresses the urgent need for clinical validation of these models so that we can use such models to develop preclinical candidate drugs more effectively and match patients to drugs in the clinical domain.

\section{Cystic fibrosis and the cystic fibrosis transmembrane conductance regulator protein}

Cystic Fibrosis (CF) is an autosomal recessive disease that affects over 70.000 people worldwide. ${ }^{1}$ It is caused by a mutation in the gene that encodes for the cystic fibrosis transmembrane conductance regulator (CFTR) protein; an epithelial ion channel that regulates chloride and bicarbonate transport throughout the body. Absent or impaired function of this protein leads to abnormal ion and water transport in multiple organs such as lungs, pancreas, gastro-intestinal tract, the male reproductive system and sweat glands. ${ }^{1-4}$ There is no cure for this multi organ disease resulting in a current median age of death of 31 years, with lung disease as the primary cause of morbidity and mortality. ${ }^{5}$ Over 2000 CFTR variants are known, of which approximately 1700 are expected to reduce CFTR function to an extent that it leads to cystic fibrosis. ${ }^{6,7}$ Based on the functional defect in the CFTR protein these mutations can be divided into seven different classes (Table 1 and Fig. 1). ${ }^{3}$ Within these classes, mutations can be found that are associated with relatively high residual function and therefore also a milder disease course at population level.

Table 1. Gene mutations divided into seven different classes.

\begin{tabular}{|c|c|c|c|c|c|c|}
\hline CLASS I & CLASS II & CLASS III & CLASS IV & CLASS V & CLASS VI & CLASS VII \\
\hline $\begin{array}{l}\text { No protein } \\
\text { production }\end{array}$ & $\begin{array}{l}\text { No } \\
\text { trafficking of } \\
\text { the protein } \\
\text { to the cell } \\
\text { membrane }\end{array}$ & $\begin{array}{l}\text { Impaired } \\
\text { channel } \\
\text { gating }\end{array}$ & $\begin{array}{l}\text { Decreased } \\
\text { channel } \\
\text { conductance }\end{array}$ & $\begin{array}{l}\text { Less protein } \\
\text { at the cell } \\
\text { membrane }\end{array}$ & $\begin{array}{l}\text { Less stable } \\
\text { protein } \\
\text { at the cell } \\
\text { membrane }\end{array}$ & $\begin{array}{l}\text { No mRNA } \\
\text { and thus no } \\
\text { protein }\end{array}$ \\
\hline
\end{tabular}




\section{CFTR modulators}

In the last years new treatment-modalities for CF have been developed. These new treatments target the CFTR protein dysfunction itself instead of treating the symptoms of reduced CFTR function, and are therefore called CFTR modulators. These modulators are developed to directly bind to CFTR and modify its 3D conformation so that the drugbound form of CFTR regains (a part of) its function. Currently, the modulators that are clinically available for treatment have two modes of actions: potentiating or correcting the CFTR protein.

\section{CFTR potentiators}

The CFTR protein can transport ions by opening and closing of the channel, which is called gating. Gating is driven by binding of adenosine triphosphate (ATP) to the nucleotide binding domains (NBD's) of the CFTR protein after cyclic adenosine monophosphate (CAMP)-mediated phosphorylation of the regulatory CFTR R-domain. ${ }^{8}$ CFTR potentiators increase the open probability of CFTR mutants that can reach the epithelial-cell surface but are defective in channel opening. ${ }^{9-11}$ Ivacaftor (VX-770 or Kalydeco ${ }^{\circledR}$ ) is the first registered CFTR modulator and has shown to improve CFTR function in patients with different class III and IV mutations. ${ }^{12-14}$ Clinical trials in which patients are treated with ivacaftor showed a clear improvement in multiple clinical outcome parameters, which makes it the first treatment that successfully targets the defective protein in CF. Besides the registered drug ivacaftor, other compounds such as the natural food supplements curcumin and genistein are also able to potentiate the CFTR protein. Like ivacaftor, curcumin has shown to activate CFTR channels independent of ATP, while genistein activates CFTR in an ATP-dependent way. ${ }^{15-18}$

\section{CFTR correctors}

CFTR correctors increase the amount of CFTR at the cell surface by improving the trafficking of mutant CFTR to the cell surface. ${ }^{19-21}$ Currently two CFTR correctors are registered in combination with the potentiator ivacaftor. These combination drugs can be used for treatment of patients homozygous for the F508del (class II) mutation, which is the most common CFTR mutation. Orkambi® is a combination of the corrector lumacaftor (VX-809) and ivacaftor and Symkevi® is a combination of the corrector tezacaftor (VX661) and ivacaftor. The latter has an improved safety profile and better pharmacokinetic properties compared to treatment with lumacaftor-ivacaftor and has also been registered for treatment of patients with 26 other mutations..$^{21,22}$

Second generation modulators consist of two correctors that target different sites within CFTR and restore CFTR function in an additive or synergistic fashion. To maximize effects, the two correctors are combined with ivacaftor, and a first triple therapy (Trikafta ${ }^{\circledR}$ a 
combination of elexacaftor (VX-445) and tezacaftor-ivacaftor) has recently been approved by the American food and drug administration (FDA). The result of the clinical trials in which the effect of this first triple therapy have been evaluated show promising results, also for patients that have only one copy of the F508del mutation. ${ }^{23,24}$

Figure 1. Biosynthesis of the CFTR protein and the mechanism of action of CFTR modulators

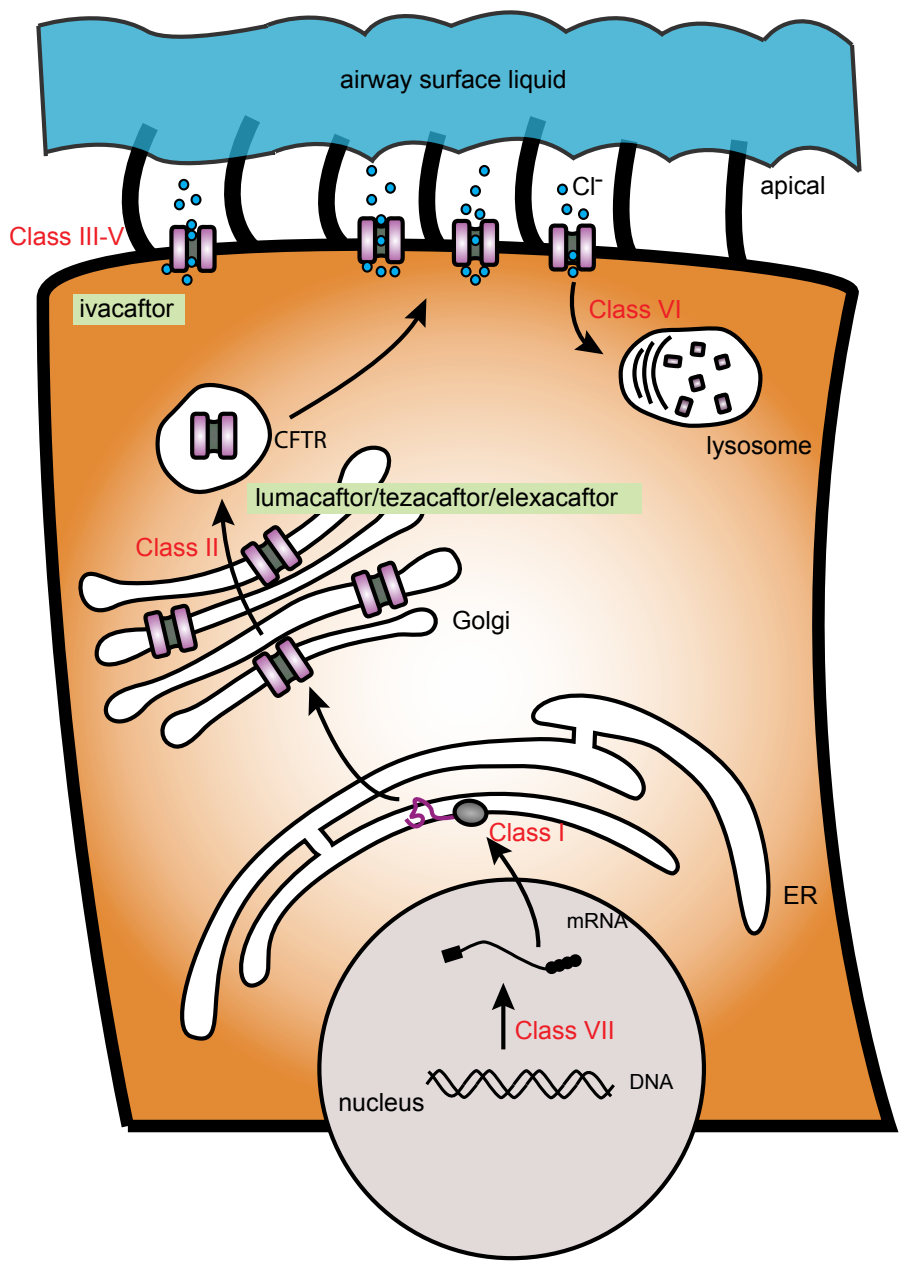




\section{Measuring CFTR function in vivo}

To evaluate the in vivo effect of a CFTR modulating treatment, different clinical measures and biomarkers have been used as parameters to (indirectly) quantitate CFTR function in individual subjects. Biomarkers of CFTR function have been used for diagnosis in patients with CF and are becoming increasingly important nowadays to evaluate the in vivo effect of CFTR modulator treatment.

\section{Biomarkers}

A biomarker is defined by the FDA as: "a characteristic that is objectively measured and evaluated as an indicator of normal biological processes, pathogenic processes, or biological responses to a therapeutic intervention". There are three biomarkers that are often used to evaluate CFTR function; sweat chloride concentration, nasal potential difference measurement and intestinal current measurement. ${ }^{26}$

Sweat chloride concentration (SCC) is an important diagnostic tool for Cystic Fibrosis. ${ }^{27}$ In the sweat gland, the diminished CFTR function leads to defective chloride absorption, which leads to an elevated chloride concentration in the sweat. ${ }^{28}$ When the SCC is low ( $<30 \mathrm{mmol} / \mathrm{L})$ CF is unlikely; a typical patient with CF has minimal CFTR function which often results in a SCC >60 mmol/L, but also a SCC between 30 and $60 \mathrm{mmol} / \mathrm{L}$ can indicate partial CFTR function. ${ }^{27,29}$ The sweat test is non-invasive and easy to perform also in young children. ${ }^{30}$ By iontophoresis with pilocarpine, localized sweating is stimulated upon which sufficient sweat can usually be collected in 30 minutes. ${ }^{31}$ In clinical trials on the effect of CFTR modulators the SCC has also been used as a biomarker to evaluate changes in CFTR function upon therapy. ${ }^{26}$ Overall, these studies show a clear decline in SCC upon clinically effective modulators. ${ }^{32}$

The nasal potential difference (NPD) measurement can be used to evaluate the function of the CFTR protein and the epithelial sodium channel (ENaC) in the respiratory epithelium in the nose. ${ }^{33}$ The cystic fibrosis foundation (CFF) accepted this research tool as a diagnostic procedure equal to SCC $>60 \mathrm{mmol} / \mathrm{L}$ in $1998 .{ }^{34,35} \mathrm{In}$ this measurement the voltage across the nasal epithelium is monitored by a double lumen catheter that is placed in the nose. During the NPD measurement changes in the voltage upon different solutions that modify the ion channel activity are monitored. ${ }^{34} \mathrm{~A}$ solution containing amiloride is used to evaluate the function of $\mathrm{ENaC}$ and CFTR function is assessed by evaluating the response to a chloride-free solution and isoproterenol.35,36 To perform a NPD measurement specialized equipment and training is necessary and the patient has to sit still for more than one hour. This makes it a more difficult measurement to perform as compared to SCC, especially in young children. Some clinical studies on CFTR modulators have used NPD as an outcome measurement and in these trials gains in CFTR activity have been observed for clinically effective CFTR modulators. ${ }^{26,37}$ 
Intestinal current measurement (ICM) is an ex vivo measurement that evaluates trans epithelial anion transport in rectal biopsies and can therefore be used to evaluate the function of the CFTR protein. To study ion transport, rectal biopsies are placed in Ussing chambers and changes in the short circuit current (ISC) upon different compounds that stimulate the ion channels (including CFTR), are monitored. ${ }^{38}$ The ICM measurement also has been validated as a diagnostic tool to discriminate CF from non-CF and shows a correlation with clinical outcome parameters. ${ }^{39-42}$ As for NPD, a center needs specialized equipment and training to perform an ICM. Also it is difficult to repeat a measurement due to the need for fresh biopsies to perform an ICM. ${ }^{38}$ Studies on the clinical effect of CFTR modulators also show increased CFTR function in the ICM of biopsies collected after treatment. ${ }^{43-45}$

\section{Clinical outcome parameters}

Since CF is a multi-organ disease, several clinical parameters can be used as a surrogate to (indirectly) evaluate CF disease and CFTR function. However, not only the function of the CFTR protein itself has an effect on the degree of organ system dysfunction, but also environmental and stochastic factors play an important role. ${ }^{1}$

Forced expiratory volume in one second (FEV1) is a clinical parameter that is often used to evaluate the function of the lungs. In many studies on the effect of CFTR modulators FEV1 is the primary outcome measurement to evaluate the clinical effect of the modulator. Recently, alternative methods such as the lung clearance index (LCI) have been developed in the search for more sensitive methods to detect lung disease in an earlier stage as compared to the $\mathrm{FEV}_{1}{ }^{46-48}$ Pulmonary disease can also be evaluated by imaging of the lungs and calculating chest computed tomography (CT) scores as a quantitative measure of airway disease..$^{49}$

A parameter that is often used to evaluate gastro intestinal disease, including malabsorption of fat, is the body mass index (BMI). Moreover, fecal elastase can be used to study exocrine pancreas function, which is affected in most patients with CF. ${ }^{50,51}$

The cystic fibrosis questionnaire (CFQ) is a patient reported outcome and a specific questionnaire to measure health related quality of life in Cystic fibrosis patients. ${ }^{52}$ The CFQ has been validated and translated in 38 languages which makes it a valuable tool to evaluate the quality of life, as well as the effect of treatment on quality of life..$^{53}$ It has been designed to assess evaluate the quality of life in different domains of functioning such as the respiratory, gastro-intestinal, emotional and social domain. 
Figure 2. Biomarkers and clinical endpoints to evaluate CFTR function

\section{Biomarkers}

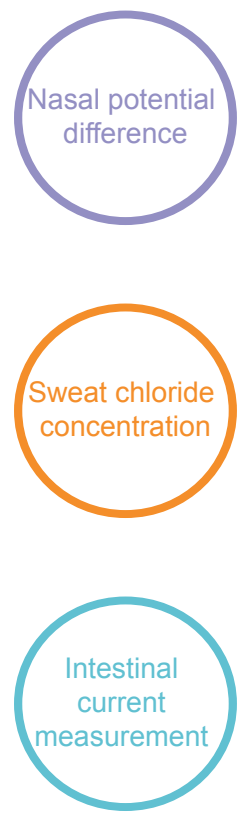

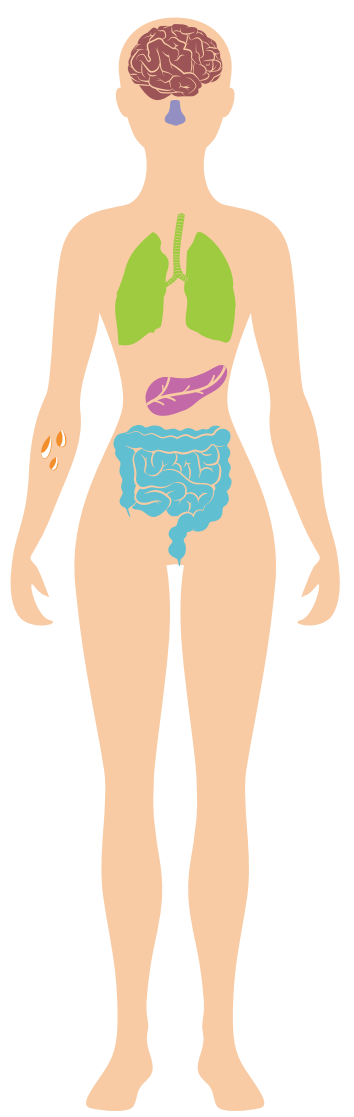

Clinical outcome parameters
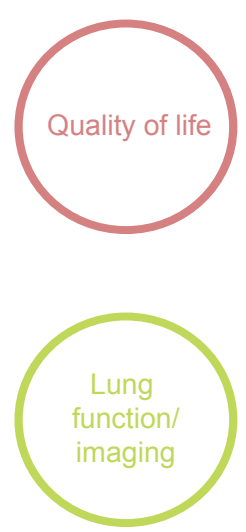

Fecal elastase

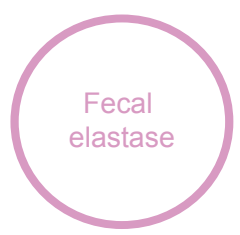

Body mass index 


\section{Intestinal organoids as a readout for CFTR function}

Recently, a fluid secretion test in intestinal organoids was developed as a new tool to evaluate the CFTR function in vitro. Organoids can be generated out of stem cells isolated from a single rectal biopsy and can be cultured unlimitedly. Organoids can be stored in living biobanks which also enables future use of these cultures. ${ }^{54}$ Rapid swelling of the organoid is observed after adding forskolin to the organoids of healthy controls, but is diminished or absent in the organoids of patients with CF (Fig. 3). This forskolin induced swelling (FIS) is fully CFTR dependent and might not only be used to evaluate the residual CFTR function but also the response of an individual patient to a CFTR modulating compound. ${ }^{54}$

Figure 3. Forskolin induced swelling in intestinal organoids as readout for CFTR function

a.
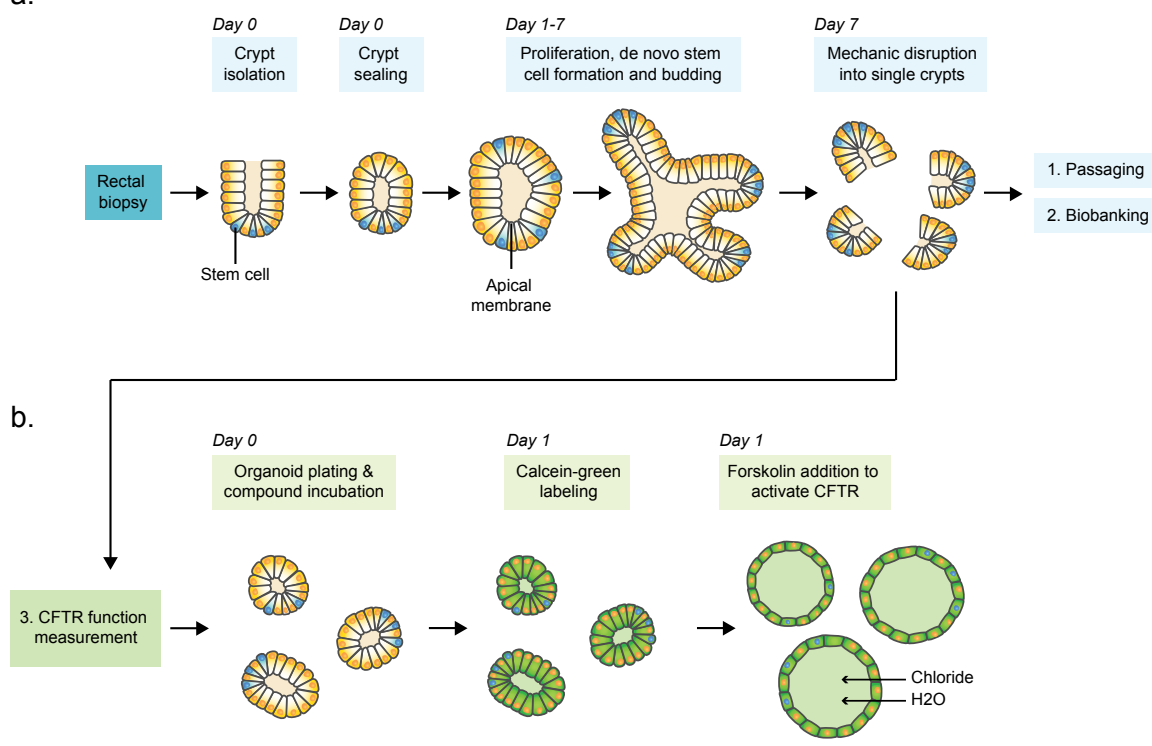

(a) Stem cells isolated from rectal biopsies are cultured into intestinal organoids which can be passaged and biobanked after disruption.

(b) Forskolin induced swelling with and without CFTR modulating compounds can be evaluated after calcein-green labeling. 


\section{Aim and thesis outline}

In this thesis we aim to evaluate the in vitro FIS response of intestinal organoids with and without different CFTR modulating drugs, and the relation with in vivo clinical endpoints that inform on CFTR function and/or disease features. By evaluating the relation between in vitro and in vivo measurements, we will get more information on the ability to use the organoid model:

I. to screen for new and effective CFTR modulating drugs for patients with different CFTR mutations

II. as a tool for personalized treatment with CFTR modulators, especially in patients with a rare CFTR mutation.

In part I of this thesis we evaluate the clinical effect of CFTR modulators that were found effective in the intestinal organoid model in a group of patients with a specific CFTR mutation. In chapter $\mathbf{2}$ we evaluate the clinical effect of the B2-adrenergic receptor agonist salbutamol ${ }^{\circledR}$ in a group of CF patients that have a mutation associated with residual CFTR function. In chapter $\mathbf{3}$ and chapter $\mathbf{4}$ we evaluate the clinical effect of (combinations of) the natural food supplements curcumin and genistein and the drug ivacaftor in patients that have at least one S1251N mutation. Finally, in chapter $\mathbf{5}$ we evaluate the clinical effect of the drug Orkambi® that was repurposed for treatment of patients with an A455E mutation, based on measurements in intestinal organoids.

In part II of this thesis we focus on the relation between in vitro FIS and in vivo CFTR function of individual patients, regardless of the patients' mutation or treatment. In chapter 6 we evaluate the relation between residual CFTR function measured in organoids and clinical outcome parameters, in a group of patients homozygous for the F508del mutation. In chapter 7 we not only evaluate the correlation between change in FIS upon CFTR modulating drugs and the clinical response in groups of patients with the same mutation, we also show the first successful selection of two patients with a very rare CFTR mutation (G1249R) for treatment with the drug ivacaftor ${ }^{\circledR}$. The correlation between organoid and clinical response to a CFTR modulator for an individual patient was further evaluated in chapter $\mathbf{8}$, in which we calculate predictive values of the organoid response for the clinical response of an individual patient. Finally, in chapter 9 we discuss the answers to our research questions and review the strengths and limitations of the intestinal organoid model. 


\section{REFERENCES}

1. Cutting GR. Cystic fibrosis genetics: from molecular understanding to clinical application. Nat Rev Genet. 2014;(November).

2. Sosnay PR, Siklosi KR, Van Goor F, et al. Defining the disease liability of variants in the cystic fibrosis transmembrane conductance regulator gene. Nat Genet. 2013;45(10):11601167.

3. De Boeck K, Amaral MD. Progress in therapies for cystic fibrosis. Lancet Respir Med. April 2016.

4. Riordan JR. CFTR Function and Prospects for Therapy. Annu Rev Biochem. 2008;77(1):701726.

5. Cystic Fibrosis Foundation Patient Registry 2017 Annual Data Report.; 2018.

6. https://cftr2.org/.

7. http://www.genet.sickkids.on.ca/.

8. Hwang T-C, Sheppard DN. Gating of the CFTR CI- channel by ATP-driven nucleotidebinding domain dimerisation. J Physiol. 2009;587(Pt 10):2151-2161.

9. Van Goor F, Hadida S, Grootenhuis PDJ, et al. Rescue of CF airway epithelial cell function in vitro by a CFTR potentiator, VX-770. Proc Natl Acad Sci. 2009;106(44):18825-18830.

10. $Y u H_{1}$ Burton B, Huang C-J, et al. Ivacaftor potentiation of multiple CFTR channels with gating mutations. J Cyst Fibros. 2012;11(3):237-245.

11. Accurso FJ, Rowe SM, Clancy JP, et al. Effect of VX-770 in persons with cystic fibrosis and the G551D- CFTR mutation. N Engl J Med. 2010;363(21):1991-2003.

12. Ramsey BW, Davies J, McElvaney NG, et al. A CFTR potentiator in patients with cystic fibrosis and the G551D mutation. N Engl J Med. 2011;365(18):1663-1672.

13. De Boeck K, Munck A, Walker $S$, et al. Efficacy and safety of ivacaftor in patients with cystic fibrosis and a non-G551D gating mutation. J Cyst Fibros. 2014;13(6):674-680.

14. Moss RB, Flume PA, Elborn JS, et al. Efficacy and safety of ivacaftor in patients with cystic fibrosis who have an Arg117His-CFTR mutation: a double-blind, randomised controlled trial. Lancet Respir Med. 2015;3(7):524-533

15. Melin P, Thoreau V, Norez C, Bilan F, Kitzis A, Becq F. The cystic fibrosis mutation G1349D within the signature motif LSHGH of NBD2 abolishes the activation of CFTR chloride channels by genistein. Biochem Pharmacol. 2004;67(12):2187-2196.

16. Wang W, Bernard K, Li G, Kirk KL. Curcumin opens cystic fibrosis transmembrane conductance regulator channels by a novel mechanism that requires neither ATP binding nor dimerization of the nucleotide-binding domains. J Biol Chem. 2007;282(7):4533-4544.

17. Eckford PDW, Li C, Ramjeesingh M, Bear CE. Cystic fibrosis transmembrane conductance regulator (CFTR) potentiator $\mathrm{VX}-770$ (ivacaftor) opens the defective channel gate of mutant CFTR in a phosphorylation-dependent but ATP-independent manner. J Biol Chem. 2012;287(44):36639-36649.

18. Sohma Y, Yu Y-C, Hwang T-C. Curcumin and genistein: the combined effects on disease-associated CFTR mutants and their clinical implications. Curr Pharm Des. 2013;19(19):3521-3528.

19. Wainwright CE, Elborn JS, Ramsey BW, et al. Lumacaftor-Ivacaftor in patients with cystic fibrosis homozygous for Phe508del CFTR. N Engl J Med. May 2015.

20. Elborn JS, Ramsey BW, Boyle MP, et al. Efficacy and safety of lumacaftor/ivacaftor combination therapy in patients with cystic fibrosis homozygous for Phe508del CFTR by pulmonary function subgroup: a pooled analysis. Lancet Respir Med. 2016;4(8):617-626.

21. Taylor-Cousar JL, Munck A, McKone EF, et al. Tezacaftor-Ivacaftor in patients with cystic fibrosis homozygous for Phe508del. N Engl J Med. November 2017:NEJMoa1709846. 
22. Rowe SM, Daines C, Ringshausen FC, et al. Tezacaftor-Ivacaftor in residual-function heterozygotes with cystic fibrosis. N Engl J Med. November 2017:NEJMoa1709847.

23. Heijerman HGM, McKone EF, Downey DG, et al. Efficacy and safety of the elexacaftor plus tezacaftor plus ivacaftor combination regimen in people with cystic fibrosis homozygous for the F508del mutation: a double-blind, randomised, phase 3 trial. Lancet. October 2019.

24. Middleton PG, Mall MA, Dřevínek P, et al. Elexacaftor-Tezacaftor-Ivacaftor for Cystic Fibrosis with a Single Phe508del Allele. N Engl J Med. 2019;381(19):1809-1819.

25. Hickey AH, Mansour HM. Inhalation Aerosols: Physical and Biological Basis for Therapy. Third edit. (Hickey, A. J. and Mansour HM, ed.). London: CRC Press/Taylor \& Francis/ Informa Healthcare; 2019.

26. Muhlebach MS, Clancy J, Heltshe SL, et al. Biomarkers for cystic fibrosis drug development. J Cyst Fibros. 2016;15(6):714-723.

27. Vermeulen F, Lebecque P, De Boeck K, Leal T. Biological variability of the sweat chloride in diagnostic sweat tests: A retrospective analysis. J Cyst Fibros. December 2016.

28. Caudri D, Zitter D, Bronsveld I, Tiddens H. Is sweat chloride predictive of severity of cystic fibrosis lung disease assessed by chest computed tomography? Pediatr Pulmonol. June 2017.

29. Lebecque P, Leal T, De Boeck C, Jaspers M, Cuppens H, Cassiman J-J. Mutations of the cystic fibrosis gene and intermediate sweat chloride levels in children. Am J Respir Crit Care Med. 2002;165(6):757-761.

30. Vermeulen F, Le Camus C, Davies JC, Bilton D, Milenković D, De Boeck K. Variability of sweat chloride concentration in subjects with cystic fibrosis and G551D mutations. J Cyst Fibros. 2017;16(1):36-40.

31. GIBSON LE, COOKE RE. A test for concentration of electrolytes in sweat in cystic fibrosis of the pancreas utilizing pilocarpine by iontophoresis. Pediatrics. 1959;23(3):545-549.

32. Fidler MC, Beusmans J, Panorchan P, Van Goor F. Correlation of sweat chloride and percent predicted FEV1 in cystic fibrosis patients treated with ivacaftor. J Cyst Fibros. October 2016.

33. Rowe SM, Liu B, Hill A, et al. Optimizing nasal potential difference analysis for CFTR modulator development: assessment of ivacaftor in CF subjects with the G551D-CFTR mutation. Boyaka PN, ed. PLoS One. 2013;8(7):e66955.

34. Solomon GM, Bronsveld I, Hayes K, et al. Standardized Measurement of Nasal Membrane Transepithelial Potential Difference (NPD). J Vis Exp. 2018;(139).

35. Naehrlich L, Ballmann M, Davies J, et al. Nasal potential difference measurements in diagnosis of cystic fibrosis: an international survey. J Cyst Fibros. 2014;13(1):24-28.

36. Knowles M, Gatzy J, Boucher R. Increased bioelectric potential difference across respiratory epithelia in cystic fibrosis. N Engl J Med. 1981;305(25):1489-1495.

37. Accurso FJ, Van Goor F, Zha J, et al. Sweat chloride as a biomarker of CFTR activity: Proof of concept and ivacaftor clinical trial data. J Cyst Fibros. 2014;13(2).

38. de Jonge $H R$, Ballmann $M$, Veeze $H$, et al. Ex vivo CF diagnosis by intestinal current measurements (ICM) in small aperture, circulating Ussing chambers. J Cyst Fibros. 2004;3:159-163.

39. Clancy JP, Szczesniak RD, Ashlock MA, et al. Multicenter intestinal current measurements in rectal biopsies from CF and non-CF subjects to monitor CFTR function. Butterworth MB, ed. PLoS One. 2013;8(9):e73905.

40. Mall M, Hirtz S, Gonska T, Kunzelmann K. Assessment of CFTR function in rectal biopsies for the diagnosis of cystic fibrosis. J Cyst Fibros. 2004;3:165-169.

41. Hirtz S, Gonska T, Seydewitz HH, et al. CFTR Cl- channel function in native human colon correlates with the genotype and phenotype in cystic fibrosis. Gastroenterology. 2004;127(4):1085-1095. 
42. Sousa M, Servidoni MF, Vinagre AM, et al. Measurements of CFTR-Mediated Cl- Secretion in Human Rectal Biopsies Constitute a Robust Biomarker for Cystic Fibrosis Diagnosis and Prognosis. Hartl D, ed. PLoS One. 2012;7(10):e47708.

43. Graeber SY, Dopfer C, Naehrlich L, et al. Effects of Lumacaftor/lvacaftor therapy on CFTR function in Phe508del homozygous patients with cystic fibrosis. Am J Respir Crit Care Med. January 2018:rccm.201710-19830C.

44. Graeber SY, Hug MJ, Sommerburg O, et al. Intestinal Current Measurements Detect Activation of Mutant CFTR in Patients with Cystic Fibrosis with the G551D Mutation Treated with Ivacaftor. Am J Respir Crit Care Med. 2015;192(10):1252-1255.

45. Masson A, Schneider-Futschik EK, Baatallah N, et al. Predictive factors for lumacaftor/ ivacaftor clinical response. J Cyst Fibros. December 2018.

46. Gustafsson PM, Aurora P, Lindblad A. Evaluation of ventilation maldistribution as an early indicator of lung disease in children with cystic fibrosis. Eur Respir J. 2003;22(6):972-979.

47. Aurora P. Multiple-breath inert gas washout test and early cystic fibrosis lung disease. Thorax. 2010;65(5):373-374.

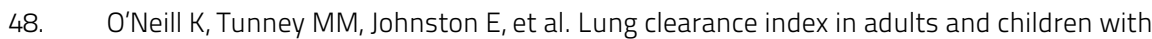
cystic fibrosis. Chest. July 2016.

49. Rosenow T, Oudraad MCJ, Murray CP, et al. PRAGMA-CF. A Quantitative Structural Lung Disease Computed Tomography Outcome in Young Children with Cystic Fibrosis. Am J Respir Crit Care Med. 2015;191(10):1158-1165.

50. Bodewes FAJA, Verkade HJ, Wilschanski M. Gastroenterological endpoints in drug trials for cystic fibrosis. Pediatr Pulmonol. July 2016.

51. Dutch Cystic Fibrosis Foundation. Nederlandse cystic fibrosis registratie 2018. 2019.

52. Gee L, Abbott J, Conway SP, Etherington C, Webb AK. Development of a disease specific health related quality of life measure for adults and adolescents with cystic fibrosis. Thorax. 2000;55(11):946-954.

53. Quittner AL, Buu A, Messer MA, Modi AC, Watrous M. Development and Validation of the Cystic Fibrosis Questionnaire in the United States. Chest. 2005;128(4):2347-2354.

54. Dekkers JF, Wiegerinck $\mathrm{CL}$, de Jonge HR, et al. A functional CFTR assay using primary cystic fibrosis intestinal organoids. Nat Med. 2013;19(7):939-945. 


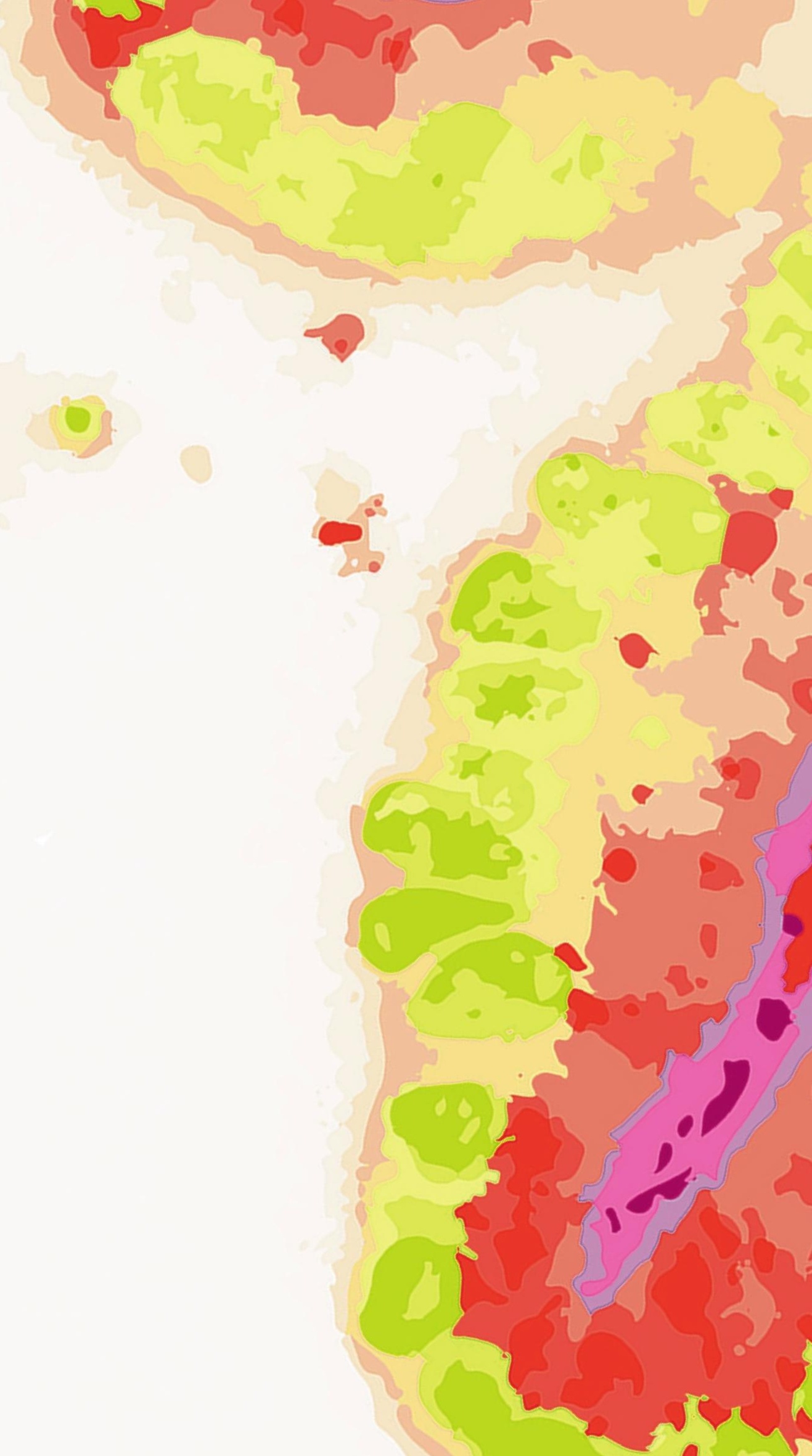




\section{CHAPTER \\ 2}

\section{ß2-ADRENERGIC RECEPTOR AGONISTS ACTIVATE CFTR IN INTESTINAL ORGANOIDS AND SUBJECTS WITH CYSTIC FIBROSIS}

Lodewijk A.W. Vijftigschild*, Gitte Berkers* Johanna F. Dekkers\#, Domenique D. Zomer-van Ommen\#, Elizabeth Matthes, Evelien Kruisselbrink, Annelotte M. Vonk, Chantal E. Hensen, Sabine Heida-Michel, Margot Geerdink, Hettie M. Janssens, Eduard A. van de Graaf, Inez Bronsveld, Karin M. de Winter-de Groot, Christof J. Majoor, Harry G.M. Heijerman, Hugo R. de Jonge, John W. Hanrahan, Cornelis K. van der Ent and Jeffrey M. Beekman

*\#" These authors contributed equally 


\section{ABSTRACT}

We hypothesized that people with cystic fibrosis (CF) who express cystic fibrosis transmembrane conductance regulator (CFTR) gene mutations associated with residual function may benefit from G-protein coupled receptor (GPCR)-targeting drugs that can activate and enhance CFTR function. We used intestinal organoids to screen a GPCR-modulating compound library and identified B2-adrenergic receptor agonists as the most potent inducers of CFTR function. B2-Agonist-induced organoid swelling correlated with the CFTR genotype, and could be induced in homozygous CFTR-F508del organoids and highly differentiated primary CF airway epithelial cells after rescue of CFTR trafficking by small molecules. The in vivo response to treatment with an oral or inhaled B2-agonist (salbutamol) in CF patients with residual CFTR function was evaluated in a pilot study. 10 subjects with a $\mathrm{R} 117 \mathrm{H}$ or $\mathrm{A} 455 \mathrm{E}$ mutation were included and showed changes in the nasal potential difference (NPD) measurement after treatment with oral salbutamol, including a significant improvement of the baseline potential difference of the nasal mucosa $(+6.35 \mathrm{mV}, \mathrm{p}<0.05)$, suggesting that this treatment might be effective in vivo. Furthermore, plasma that was collected after oral salbutamol treatment induced CFTR activation when administered ex vivo to organoids. This proof-of-concept study suggests that organoids can be used to identify drugs that activate CFTR function in vivo and to select route of administration. 


\section{INTRODUCTION}

The cystic fibrosis transmembrane conductance regulator (CFTR) gene encodes an apical anion channel and is mutated in subjects with cystic fibrosis (CF). ${ }^{1}$ Subjects with CF have an altered composition of many mucosal surface fluids, leading to dysfunction of the gastrointestinal and pulmonary systems as well as other organs. The most common mutation is a deletion of phenylalanine at position 508 (p.Phe508del; F508del) and is present in $\sim 90 \%$ of subjects with CF, of which $\sim 65 \%$ are F508del homozygotes (www. genet.sickkids.on.ca). CF disease expression is highly variable between subjects due to the complex relations between CFTR genotype, modifier genes and environmental factors, which are unique for each individual. ${ }^{2-6}$

Approximately 2000 CFTR mutations have been described, which are divided into different classes according to their impact on CFTR expression and function. ${ }^{7}$ Briefly, class I mutations result in no functional protein (e.g. stop codons and frameshifts), class II mutations severely affect apical trafficking (e.g. F508del), class III mutations disrupt channel regulation or gating (e.g. p.Gly551Asp; G551D), class IV mutations reduce channel conductance (e.g. p.Arg334Trp; R334W), class V mutations lead to reduced apical expression of normally functioning CFTR (e.g. p.Ala455Glu; A455E) and class VI mutations accelerate CFTR turnover at the plasma membrane. Whereas class I-III and VI mutations are generally associated with no or very limited residual function, some residual function is associated with class IV and $\mathrm{V}$ mutations and milder disease phenotype such as CFTRA455E and CFTR-R117H (p.Arg117His; shared class III and IV) (www.cftr2.org).

Novel drugs are being developed to target mutation-specific CFTR defects. The potentiator VX-770 (ivacaftor) enhances the activity of apical CFTR and was shown to provide clinical benefit for patients with CFTR gating mutations. ${ }^{8-10}$ Pharmacological repair of CFTR-F508del has proven more difficult, although encouraging phase III clinical trial results have been reported for CFTR-F508del homozygous subjects treated with a combination of ivacaftor and the corrector lumacaftor (VX-809), which partly restores trafficking of CFTR-F508del to the apical membrane. ${ }^{11,12}$ However, the therapeutic effects of these therapies are variable between subjects, and remain insufficient to fully restore CF and CFTR-related disease markers, indicating that more effective treatments are still required.

Individual CFTR function depends on endogenous signaling pathways that control its channel function. Various endogenous ligands have been identified which activate CFTR in a cyclic adenosine monophosphate (CAMP)/protein kinase A-dependent fashion. Many of these ligands (e.g. vasoactive intestinal peptide, prostaglandins and B-adrenergic stimuli) signal by binding to a G-protein coupled receptor (GPCR), which release cytosolic G-proteins that activate adenylyl cyclase to generate CAMP. ${ }^{13-15}$ While it is known that tissue-specific activity of CFTR is regulated by diverse ligands, the extent to which CFTR 
function is limited by cAMP production is not clear.

We hypothesised that CAMP-dependent signaling is a rate-limiting step for CFTR activation in vivo and that CF individuals who express alleles associated with residual function might benefit from existing drugs that stimulate cAMP. Therefore, we screened a small chemical compound library of GPCR modulators for their ability to stimulate (mutant) CFTR activity in primary rectal organoids from healthy control and CF subjects. Rectal organoids grow from intestinal stem cells and self-organise into multicellular three-dimensional structures consisting of a single epithelial layer, with the apical membrane facing a closed central lumen. ${ }^{16-18}$ Addition of forskolin, which raises CAMP, stimulates CFTR-dependent fluid secretion into the organoid lumen and induces rapid organoid swelling. ${ }^{19,20}$ Here, we provide proof-of-concept that intestinal organoids can be used as tool to identify potential drugs and route of administration for particular CF subgroups.

\section{METHODS}

\section{Human participants}

This study was approved by the Ethics Committee of the University Medical Centre Utrecht and the Erasmus Medical Center Rotterdam. Informed consent was obtained from all subjects. Organoids from healthy controls and CF subjects were generated from rectal biopsies after intestinal current measurements obtained 1) during standard CF care, 2) for diagnostic purposes or 3) during voluntary participation in studies.

\section{Materials}

The GPCR compound library, VX-809 and VX-770 were purchased from SelleckChem (Houston, TX, USA). Carvedilol, forskolin, salbutamol, salmeterol, terbutaline, epinephrine, ritodrine, dimethylsulphoxide (DMSO), N-acetylcysteine, nicotinamide and SB202190 were purchased from Sigma (St Louis, MO, USA). Formoterol was purchased from Santa Cruz Biotechnologies (Dallas, TX, USA). CFTRinh-172 was obtained from CFF Therapeutics (Chicago, IL, USA). Matrigel was purchased from BD (Franklin Lakes, NJ, USA). Calcein AM, supplements N-2 and B-27, Glutamax, advanced Dulbecco's modified Eagle medium/Ham's F-12 (DMEM/F-12), penicillin/streptomycin, hydroxyethyl piperazineethanesulfonic (HEPES) and murine epidermal growth factor (mEGF) were purchased from Life Technologies (Bleiswijk, The Netherlands). A83-01 was purchased from Tocris (Abingdon, UK). TOPflash and FOPflash were purchased from Millipore (Amsterdam, The Netherlands). 


\section{Human organoid cultures}

Rectal crypt isolation and organoid expansion was performed with some adaptations of previously described methods. ${ }^{20,21}$ Briefly, rectal biopsies were thoroughly washed with PBS and incubated in $10 \mathrm{mM}$ ethylenediaminetetraacetic (EDTA) for 90 min at $4^{\circ} \mathrm{C}$ The crypts were collected by centrifugation and suspended in 50\% Matrigel and 50\% complete culture medium (advanced DMEM/F-12 media supplemented with penicillin/ streptomycin, HEPES, GlutaMax, nutrient supplements N-2 and B-27, N-acetylcysteine, nicotinamide, mEGF, A83-01, SB202190, and 50\% Wnt3a, 20\% Rspo-1 and 10\% Noggin conditioned media) that was allowed to solidify at $37^{\circ} \mathrm{C}$ for 20 min in three droplets of 10 $\mu \mathrm{L}$ per well of a 24 -well plate. The droplets were then immersed in pre-warmed complete culture medium and cultures were expanded for at least 3 weeks before assaying CFTR function. Complete culture medium was refreshed three times per week and organoids were passaged weekly. Quality of the conditioned media was assessed by dot blots, enzyme-linked immuno sorbent assay (ELISA) and luciferase reporter constructs (TOPflash and FOPflash). ${ }^{22,23}$

\section{GPCR compound library}

The GPCR small-molecule compound library comprises 61 agonists and antagonists that target a wide range of GPCR families, including adrenergic, dopamine, opioid, serotonin, histamine and acetylcholine receptors. A complete list of chemicals in the library is given in supplemental Table S1.

\section{CFTR function measurement in organoids}

Organoids were reseeded 1 day before functional analysis in 96-well plates as described previously [20]. CFTR-F508del organoids were incubated with VX-809 (3 $\mu \mathrm{M})$ for $24 \mathrm{~h}$, as indicated in text and figure legends. Organoids were stained with Calcein green AM $(2.5 \mu \mathrm{M}) 1 \mathrm{~h}$ prior the addition of compound and each compound was tested at four different concentrations (10, 2, 0.4 and $0.08 \mu \mathrm{M})$. Forskolin $(5 \mu \mathrm{M})$ and DMSO were used as positive and negative controls, respectively. Organoid swelling was monitored for $1 \mathrm{~h}$ using a Zeiss LSM 710 confocal microscope (Zeiss, Jena, Germany). The relative increase in surface area was calculated using Volocity (version 6.1.1; PerkinElmer, Waltham, MA, USA). The area under the curve (AUC) was calculated as described previously. ${ }^{20}$ Carvedilol $(10 \mu \mathrm{M})$ was incubated for 30 min prior to stimulation and organoids were pre-treated with CFTRinh-172 $(150 \mu \mathrm{M})$ for $4 \mathrm{~h}$ to inhibit CFTR-dependent responses.

\section{Halide-sensitive yellow fluorescent protein (YFP) quenching in cystic fibrosis bronchial epithelial (CFBE) 410- cells}

CFBE410- cell lines overexpressing CFTR-F508del or CFTR-wild type (WT) were grown in $\partial$-minimal essential medium containing $8 \%$ heat-inactivated fetal calf serum, penicillin 
and streptomycin at $37^{\circ} \mathrm{C}$ in a humidified 5\% CO2 incubator as described. ${ }^{24,25}$ CFBE410cells were transduced with the ratiometric halide-sensitive pHAGFE2-YFP (46L-148Q152L)-mKate sensor for measurement of CFTR activity as described previously. ${ }^{26}$ Briefly, cells were incubated for $24 \mathrm{~h}$ with VX-809 $(10 \mu \mathrm{M})$. After 20 min stimulation in a chloride-containing buffer, the cells were washed with iodide buffer and the decrease in fluorescence was monitored using a Zeiss LSM 710 microscope for $60 \mathrm{~s}$. The rate of YFP/ mKate quenching was calculated using Prism 6 (GraphPad, La Jolla, CA, USA).

\section{Ussing chamber measurements in primary airway epithelial cells}

Primary F508del/F508del human bronchial epithelial cells from the Primary Airway Cell Biobank of the CF Translational Research Centre at McGill University were cultured at the air/liquid interface for 3 weeks and pre-treated for $24 \mathrm{~h}$ with VX-809 ( $1 \mu \mathrm{M})$. Control monolayers from the same patients were handled similarly but exposed to vehicle (0.1\% DMSO) during the pre-treatment period. For electrophysiological measurements, monolayers were mounted in Ussing chambers (EasyMount; Physiologic Instruments, San Diego, CA, USA) and voltage-clamped using a VCCMC6 multichannel current-voltage clamp (Physiologic Instruments, San Diego, CA, USA). The voltage clamp was connected to a PowerLab/8SP interface for data collection (ADInstruments, Colorado Springs, CO, USA) and analysis was performed using a PC as described previously.27 Solutions were continuously gassed and stirred with 95\% 02/5\% CO2 and were maintained at $37^{\circ} \mathrm{C}$ by circulating water bath. $\mathrm{Ag} / \mathrm{AgCl}$ reference electrodes were used to measure transepithelial voltage and pass current. Pulses ( $1 \mathrm{mV}$ amplitude, $1 \mathrm{~s}$ duration) were delivered every $90 \mathrm{~s}$ to monitor resistance. A basolateral-to-apical $\mathrm{Cl}$ - gradient was imposed and amiloride $(100 \mu \mathrm{M})$ was added on the apical side to inhibit the epithelial sodium channel (ENaC) current. Monolayers were exposed acutely to $10 \mu \mathrm{M}$ forskolin or salbutamol or to the vehicle $0.1 \% \mathrm{DMSO}$. After the short circuit current (Isc) reached a

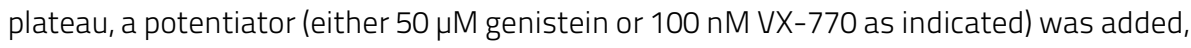
followed by CFTRinh-172 to confirm that the current responses were dependent on CFTR. Salbutamol was also assayed after pre-treatment with the antagonist carvedilol $(10 \mu \mathrm{M})$ for 30 min as a further test of receptor specificity.

\section{Pilot study with inhaled and oral salbutamol}

In this open-label phase II pilot study, 10 patients were randomly assigned to receive four times daily $200 \mu \mathrm{g}$ salbutamol per inhalation or four times daily $4 \mathrm{mg}$ salbutamol orally, for 3 consecutive days (www.trialregister.nl/trialreg/admin/rctview.asp?TC=4513). After a wash-out period of at least 4 days, patients received the opposite treatment. We included patients aged $\geq 18$ years old with a CFTR-A455E or a CFTR-R117H mutation on at least one allele of whom rectal biopsies and organoid cultures showed residual CFTR function in previous studies. ${ }^{20}$ Patients were excluded if they had an acute pulmonary exacerbation or an increased risk of side-effects of salbutamol. The primary outcome 
measures were changes in sweat chloride concentration (SCC) and changes in nasal potential difference (NPD) measurements, which are both in vivo biomarkers for CFTR function. The NPD and SCC measurements were performed according to the most recent version of the standard operating procedure of the European Cystic Fibrosis Society Clinical Trials Network (www.ecfs.eu/ctn). The results of these measurements before and after treatments with salbutamol were compared using a Wilcoxon signed-rank test. A secondary outcome measure was the CFTR-activating capacity of the patients' plasma in organoids. Therefore, whole blood was collected in sodium-heparin tubes before treatment and after the last dose of salbutamol, when the maximum concentration of salbutamol in the blood was expected (inhaled salbutamol after 30 min, oral salbutamol after 2 h; www.fk.cvz.nl). Plasma was isolated as described previously. ${ }^{28}$

\section{Patient plasma-induced organoid swelling}

Patient plasma was collected before and after treatment with salbutamol and incubated (20\% and $40 \%$ plasma) with organoids derived from subjects with CF with high residual CFTR function (R117H/F508del). Organoid swelling was monitored as described above. Reference values were generated by measurement of spiked salbutamol in $0 \%, 20 \%$ and $40 \%$ plasma.

\section{RESULTS}

\section{Screen for GPCR modulators of organoid swelling}

To identify compounds that can activate CFTR, we assessed CFTR-dependent swelling of organoids in response to 61 GPCR-modulating compounds (Fig. 1). ${ }^{20}$ As observed previously, forskolin induced rapid swelling of CFTR-WT organoids and, to a lesser extent, of VX-809-treated homozygous CFTR-F508del organoids. As expected, DMSO did not induce swelling (Fig. 1a). Swelling was expressed as the AUC for each specific condition (Fig. 1a,b). Of the 61 compounds tested, dopamine, epinephrine, ritodrine and salbutamol dose-dependently induced swelling of CFTR-WT organoids, with highest potency for ritodrine and salbutamol, and lowest potency for dopamine (Fig. 1C,d). Epinephrine, ritodrine and salbutamol are ligands for B2-adrenergic receptors, and dopamine for the dopamine receptor. At the highest dose, the response to the four compounds was comparable to the forskolin-induced swelling (Fig. 1d). In VX-809-corrected F508del homozygous organoids, swelling was dose-dependently induced by epinephrine, salbutamol and ritodrine, but not by dopamine (Fig. 1C,e). The potency was highest for salbutamol and lowest for ritodrine. High levels of salbutamol induced swelling to a similar extent as forskolin (Fig. 1d). In conclusion, B2-adrenergic receptor stimulation can potently activate CFTR-WT and drug-corrected CFTR-F508del in organoids. 
Figure 1. GPCR modulator-induced swelling of healthy control and CFTR-F508del organoids

a.

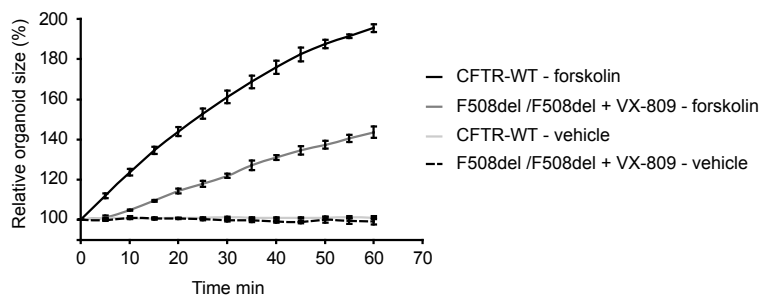

b.

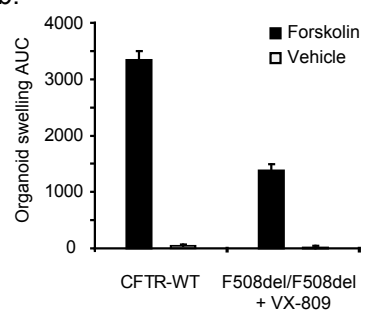

C.

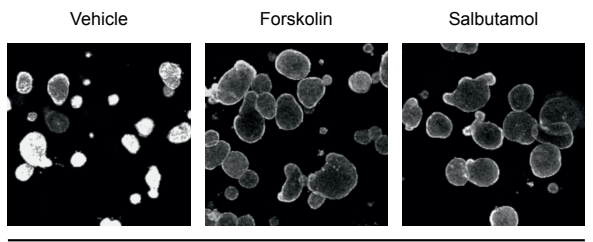

CFTR-WT

d.

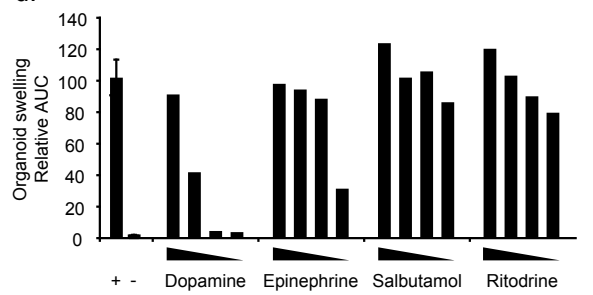

Vehicle

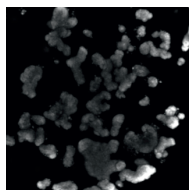

Forskolin

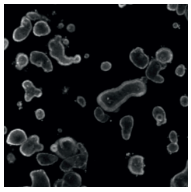

F508del /F508del + VX-809

e.

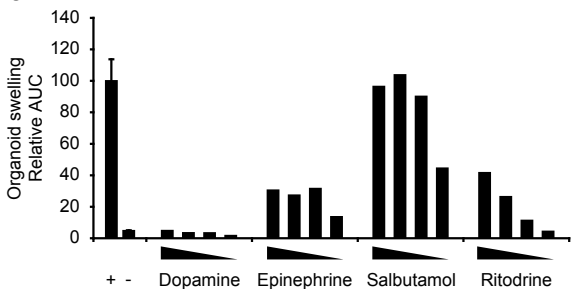

(a) CFTR WT and F508del homozygous organoids were stimulated with forskolin ( $5 \mu \mathrm{M}$ ) DMSO (vehicle, 0.05\%) and the relative increase in size was monitored over $60 \mathrm{~min}$. CFTR-F508del homozygous organoids were pre-incubated with VX-809 (3 $\mu \mathrm{M})$ for $24 \mathrm{~h}$.

(b) Quantification of the AUC of (a), baseline was set at 100\%, $\mathrm{t}=60 \mathrm{~min}$.

(c) Representative images of CFTR-WT and VX-809-corrected CFTR-F508del organoids after 60 min of stimulation with DMSO (vehicle), forskolin (5 $\mu$ M) or salbutamol $(10 \mu \mathrm{M})$. Scale bar: $200 \mu \mathrm{m}$.

(d) Positive compounds for induction of fluid secretion using CFTR-WT intestinal organoids after screening a GPCR modulator library (61 compounds). Forskolin (+) and DMSO (-) were used as positive and negative control, respectively. GPCR modulators were tested at 10,2,0.4 and $0.08 \mu \mathrm{M}$. Data are normalised to the forskolin response.

(e) Same compounds as in (d), tested on VX-809 (3 $\mu \mathrm{M})$-treated F508del homozygous organoids. 


\section{B2-Agonists robustly induce organoid swelling}

Next, we assessed B2-adrenergic receptor stimulation by short- and long-acting agonists in organoids with various CFTR mutations (Fig. 2). First, salbutamol- and ritodrineinduced swelling was confirmed in organoids derived from three individual F508del homozygous patients (Fig. 2a). As expected, robust organoid swelling was only observed after treatment with the CFTR modulators VX-770 or VX-809, and was highest upon VX-770 and VX-809 combination treatment. In line with Fig. 1, ritodrine was somewhat less potent than salbutamol and forskolin, especially for VX-809-incubated organoids. Both short-acting (ritodrine, terbutaline and salbutamol) and long-acting (formoterol, salmeterol and isoproterenol) B2-agonists induced fluid secretion. Inhibition by CFTRinh172 or carvedilol supported CFTR or ß-adrenergic receptor specificity, respectively (Fig. 2b). Forskolin- and B2-adrenergic receptor-induced swelling differed between organoids with distinct CFTR genotypes: we observed no swelling in organoids expressing two CFTRnull alleles (p.Glu60Ter and p.lle1295fs; E60X and 4015delATT), some swelling in CFTRA455E or VX-809-corrected homozygous CFTR-F508del organoids and high swelling in CFTR-R117H expressing organoids (Fig. 2C). Dose-dependencies of B2-agonist-induced swelling were independent of the CFTR genotype or VX-809-rescued F508del, and indicated that long-acting B2-agonists were most potent, whereas forskolin was least potent (Fig. 2d). Representative examples of agonist-induced swelling are indicated in Fig. 2e. Together, these data demonstrate that various B2-agonists robustly induce CFTR function in a CFTR mutation-dependent manner.

\section{Salbutamol-mediated CFTR activation in bronchial epithelial cells}

To confirm that intestinal organoid responses can be relevant for airway epithelial cells, CFTR activation by the B2-agonist salbutamol was studied in the CF airway cell line CFBE410- and primary CF human bronchial epithelial cells. First, we studied CFTRdependent iodide quenching rates in CFBE410- cells that endogenously express CFTRF508del and were previously transduced with CFTR-F508del (CFBE-F508del) or CFTR-WT (CFBE-CFTR-WT) CDNA. ${ }^{24}$ To measure CFTR-dependent iodide influx, the cells were stably transduced with a YFP/mKate sensor, as described previously. ${ }^{28}$ Quenching of the YFP signal by iodide (indicating CFTR activity) was induced by both forskolin and salbutamol in VX-809+VX-770-treated CFTR-F508del and CFTR-WT CFBE410- cells (Fig. 3a). In addition, Ussing chamber experiments revealed that salbutamol and forskolin induced a CFTR-dependent short circuit current in F508del homozygous human bronchial epithelial cells treated for $24 \mathrm{~h}$ with $\mathrm{VX}-809$ and $\mathrm{VX}-770$, but not in primary cultures without CFTRrepairing treatment (Fig. $3 b$ to d). As expected, the acute response to forskolin and salbutamol was abolished by CFTRinh-172 and the salbutamol-induced response was inhibited by carvedilol (Fig. 3c,d). To conclude, activation of modulator-repaired CFTRF508del by B2-agonists was recapitulated in respiratory cell lines and primary airway cultures. 
Figure 2. B2-Agonists induce CFTR activity

a.

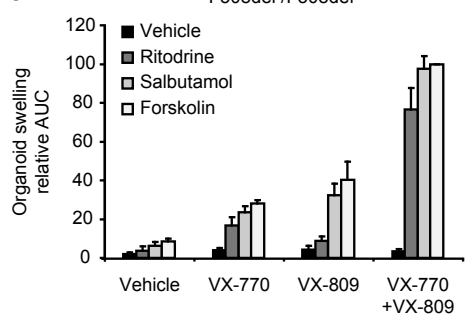

c.

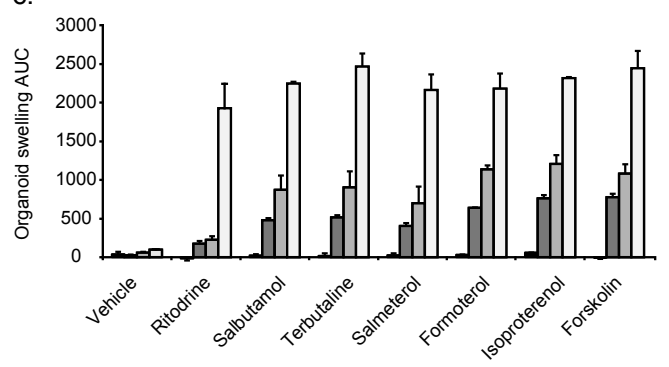

d.
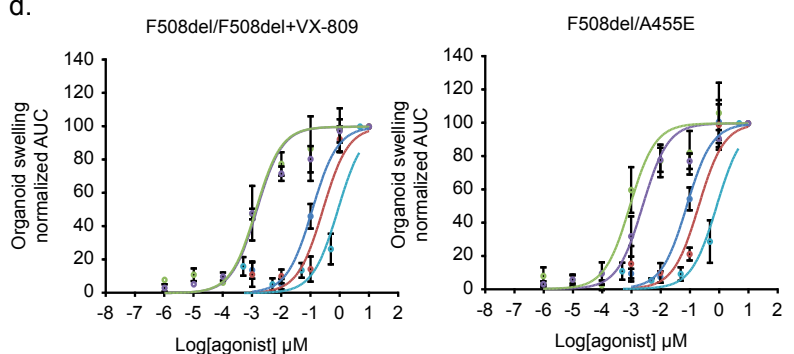

b.

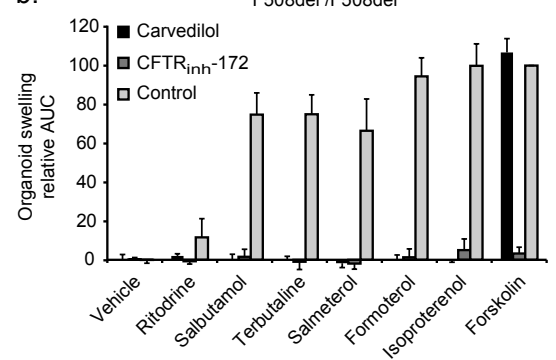

- E60X/4015delATTT+VX-809+VX-770 (n= 1)

口 F508del/A455E ( $n=2)$

F508del/F508del+VX-809 (n=3)

口 F508del/R117H (n=1) and R117H/R117H (n=1)

e.

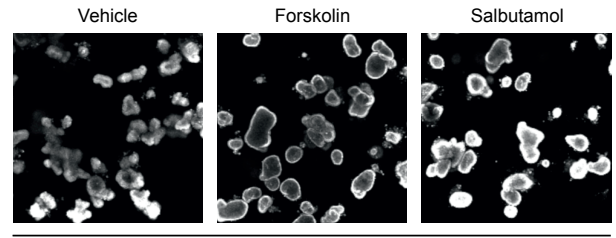

F508del/A455E
F508del/R117H

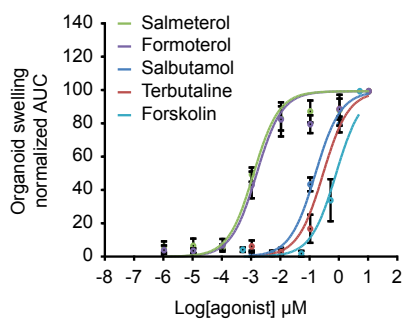

Vehicle

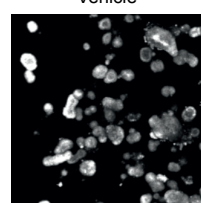

Salbutamol
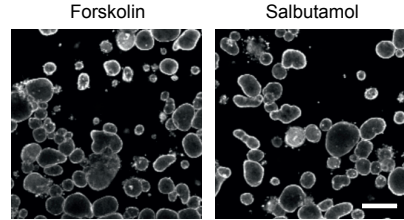

F508del/R117H 
(a) CFTR-F508del homozygous organoids were stimulated with ritodrine $(10 \mu \mathrm{M})$, salbutamol $(10 \mu \mathrm{M})$ or forskolin $(5 \mu \mathrm{M})$. VX-809 (3 $\mu \mathrm{M})$ was incubated for $24 \mathrm{~h}$ prior to stimulation. VX-770 (1 $\mu \mathrm{M})$ was added simultaneously with the stimulus. Data were normalised to the combined VX-770+VX-809+forskolin response and organoids from three patients were measured at three independent time points in duplicate. Data are presented as mean \pm standard error of the mean (SEM).

(b) VX-809 (3 $\mu \mathrm{M})$ treated CFTR-F508del organoids were incubated with CFTRinh-172 or carvedilol before stimulation.

B2-Agonists were used at $10 \mu \mathrm{M}$ and forskolin at $5 \mu \mathrm{M}$. All data were normalised to forskolin and represent mean \pm

SEM of three independent measurements in duplicate.

(c) Organoids derived from patients with different CFTR genotypes were stimulated with B2-agonists (10 $\mu \mathrm{M})$ or forskolin $(5 \mu \mathrm{M})$. n: number of patients, measured at three independent time points in duplicate (mean \pm SEM).

(d) Dose-response curves for different B2-agonists and forskolin in F508del/F508del, F508del/A455E and F508del/

$\mathrm{R} 117 \mathrm{H}$ organoids. All data were normalised to the highest concentration of stimulus and represent mean $\pm \mathrm{SEM}$ of measurements at three independent time points.

(e) Representative images of organoids expressing CFTR-F508del and either CFTR-A455E or CFTR-R117H after 60 min of stimulation with DMSO, forskolin $(5 \mu \mathrm{M})$ or salbutamol $(10 \mu \mathrm{M})$. Scale bar: $200 \mu \mathrm{m}$. Vehicle represents DMSO in all cases.

\section{Pilot study with inhaled and oral salbutamol}

To evaluate if drugs identified by screens in rectal organoids can be used to modulate CFTR function in vivo, 10 CF patients were enrolled in a study, and treated with oral and inhaled salbutamol. One patient was only treated with oral salbutamol due to increased asthma symptoms during the wash-out period of salbutamol aerosol between both treatments. The baseline characteristics of the study population are shown in Table 1. To analyze the systemic delivery of salbutamol by inhalation or oral application, we stimulated F508del/R117H and F508del/A455E organoids with plasma collected before and after in vivo treatment. Plasma collected after oral salbutamol treatment significantly induced F508del/R117H organoid swelling compared with the plasma collected before treatment or after aerosol administration of salbutamol, indicating that plasma concentrations of salbutamol were highest after oral treatment (Fig. 4a,b). Spiking of pure salbutamol in pooled plasma of subjects before treatment indicated that active salbutamol levels were below detection levels upon aerosol treatment and detectable but low after oral treatment, amounting to $\sim 5 \mathrm{nM}$ (Fig. 4C). After correcting for $40 \%$ subject plasma samples, circulating salbutamol levels after oral treatment on average reached levels $\sim 12.5 \mathrm{nM}$. To monitor in vivo modulation of CFTR function, SCC and NPD measurements were performed before and after 3 days of treatment with salbutamol. An overview of the changes in the SCC and NPD values is given in Table 2 and Table 3. Consistent with the outcome of the salbutamol bioassay showing low (oral treatment) or undetectable (aerosol treatment) levels of salbutamol in the plasma (nanomolar range), the only significant change in NPD was seen upon oral (but not aerosol) treatment, i.e. the median baseline potential changed significantly (by $6.4 \mathrm{mV}$ ) in the direction of reduced sodium absorption, indicative of an improved CFTR function (Table 2). However, we did not observe any significant changes in other NPD parameters nor in levels of sweat chloride (Table 2 and Table 3 and online supplemental Table S2 and supplemental Table S3). 
Figure 3. B2-Agonist-induced CFTR activation in bronchial epithelial cells

a.

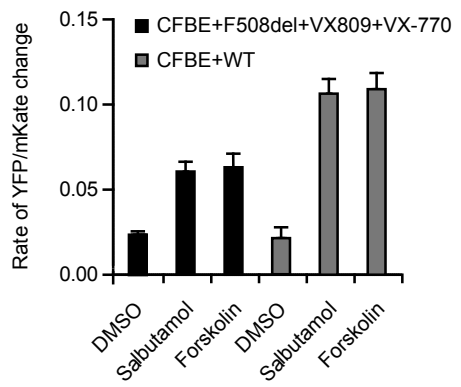

c.

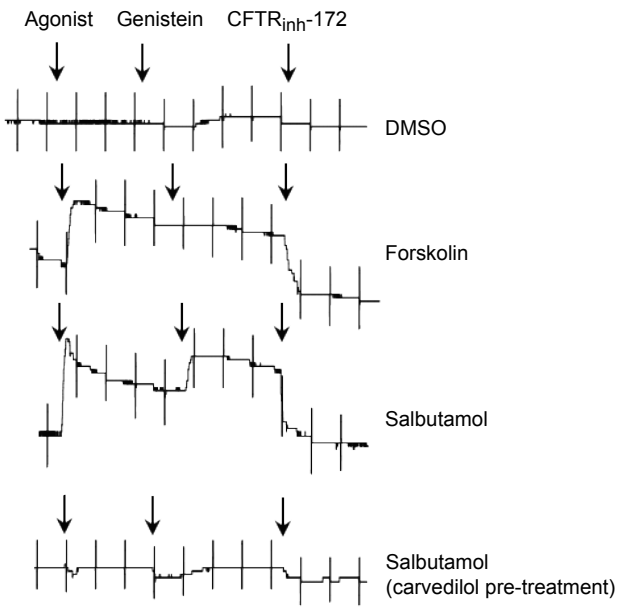

b.
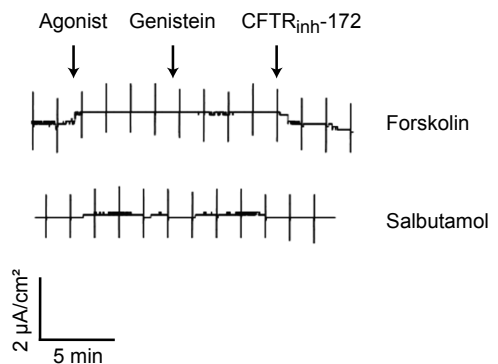

d.

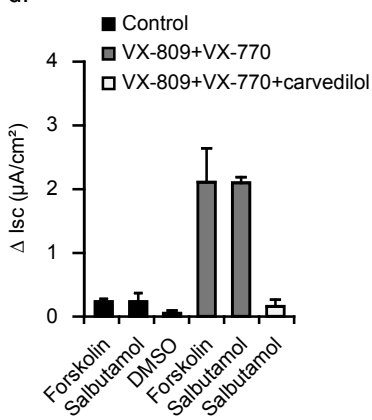

(a) CFTR activity in CFBE410- cells overexpressing CFTR-F508del or CFTR-WT, and stably expressing YFP/mKate, using a YFP quenching assay. CFBE410- cells were pre-incubated for $24 \mathrm{~h}$ with VX-809 $(10 \mu \mathrm{M})$, and stimulated with forskolin $(25 \mu \mathrm{M})$ and $V X-770(10 \mu \mathrm{M})$ or salbutamol $(10 \mu \mathrm{M})$ and $V X-770(10 \mu \mathrm{M})$ for 20 min prior to addition of iodide. Data are presented as mean \pm SEM and are representative of three independent experiments.

(b) Highly differentiated primary CFTR-F508del bronchial epithelial cells cultured at the air/liquid interface were analysed in Ussing chamber experiments. Representative traces of primary CF cells untreated with CFTR modulator drugs and exposed acutely to forskolin or salbutamol. Constant current pulses used to monitor transepithelial resistance cause the vertical deflections.

(c) Representative Ussing chamber tracings for VX-809 (1 $\mu \mathrm{M})$ - and VX-770 (100 nM)-treated primary CF bronchial epithelial cells, stimulated acutely with DMSO, forskolin $(10 \mu \mathrm{M})$ or salbutamol $(10 \mu \mathrm{M})$. Scaling is identical to (b). (d) Quantification of (b and c). Data are presented as mean \pm SEM and are representative of three independent experiments. 
Table 1. Baseline characteristics of the 10 subjects enrolled in the pilot study

\begin{tabular}{|ll|}
\hline Age in years & $38.5(31.5-49.0)$ \\
\hline Male & $4(40)$ \\
\hline Body mass index $\mathrm{kg} \cdot \mathrm{m}-2$ & $22.28(20.38-28.16)$ \\
\hline FEV1 \% pred (range) & $62.0(44.8-84.8)(31-109)$ \\
\hline CFTR genotype & $9(90)$ \\
\hline F508del/A455E & $1(10)$ \\
\hline F508del/R117H & \\
\hline
\end{tabular}

Data are presented as median (interquartile range) or $\mathrm{n}(\%)$, unless otherwise stated.

CFTR, cystic fibrosis transmembrane conductance regulator; FEV1, forced expiratory volume in 1 second

Adverse events that were reported during treatment with salbutamol appeared higher for oral salbutamol and are summarised in Table 4. The data of this pilot study tentatively indicate that oral, but not inhaled, treatment with ß2-agonists may slightly improve residual CFTR function in nasal epithelium in vivo, but failed to further improve CFTR function in the sweat duct.

\section{DISCUSSION}

The purpose of this study was to generate proof-of-concept that organoid-based measurements can be used to identify approved drugs that may modulate CFTR function in vivo. In this study, CFTR function measurements in organoids were applied to 1) prioritize potential drugs out of multiple candidates, 2) identify and verify subjects with potential responsive CFTR variants, and 3) study the optimal route of administration of the drug. As a whole, the results of the pre-clinical and clinical studies together indicate that organoidbased measurement can aid in designing clinical studies for subjects with CF.

We selected B2-agonists from 61 compounds that can modulate GPCR signaling, which are known activators of CFTR and anion transport. Surprisingly, the potency of B2agonists to stimulate CFTR function was equal or greater than forskolin, which directly stimulates adenylyl cyclase downstream of GPCR, as observed in both primary intestinal and airway cells (Fig. 2 and Fig. 3). 13,29 The formation of macromolecular complexes 
Figure 4. Plasma-induced organoid swelling

a.

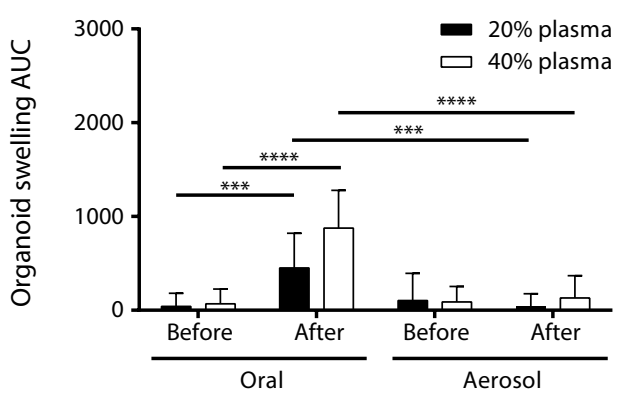

b.

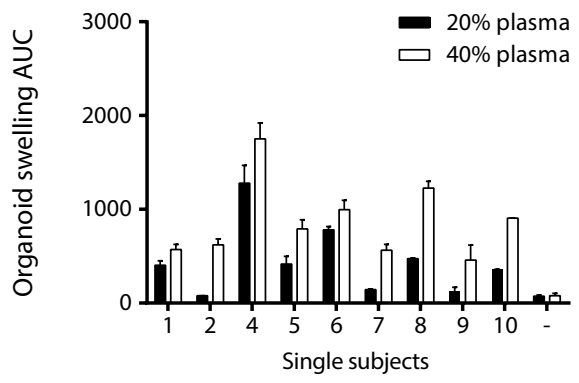

c.

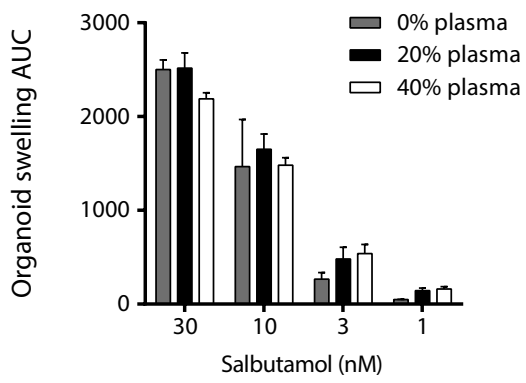

(a) CFTR-F508del/R117H organoids were stimulated with $20 \%$ and $40 \%$ plasma of each subject ( $\mathrm{n}=9$ ) both before and after treatment via both aerosol and oral administration of salbutamol. AUC: $t=60$ min. Outcomes of subjects were pooled to compare aerosol with oral administration. Data are presented as mean, \pm standard deviation (SD). Paired t-tests were performed to determine significance. ${ }^{* * *}$ : $p<0.001 ; * * * *$ : $p<0.0001$.

(b) Data from plasma samples after oral treatment of (a) are presented per subject. Data are presented as mean \pm SD. -: pooled plasma of all donors before treatment.

(c) To quantify the concentrations of salbutamol in the plasma samples of the subjects, F508del/R117H organoids were stimulated with known concentrations of salbutamol spiked in $0 \%, 20 \%$ and $40 \%$ plasma.

between B2-adrenergic receptors and CFTR may enable this efficient coupling of signals from B2-adrenergic receptors to CFTR. ${ }^{30}$ The lack of CFTR activation by other compounds in this library most likely reflects the absence of their cognate receptors or their inability to induce sufficient CFTR-activating signals or coupling these to CFTR.

Measurement of in vivo CFTR function enhanced by exogenous activators such as B2agonists requires a different approach as compared with direct CFTR protein restoring drugs such as $V X-770$ and $V X-809$. It relies on the ability of exogenous B2-agonists to 
Table 2. SCC and NPD responses to oral salbutamol

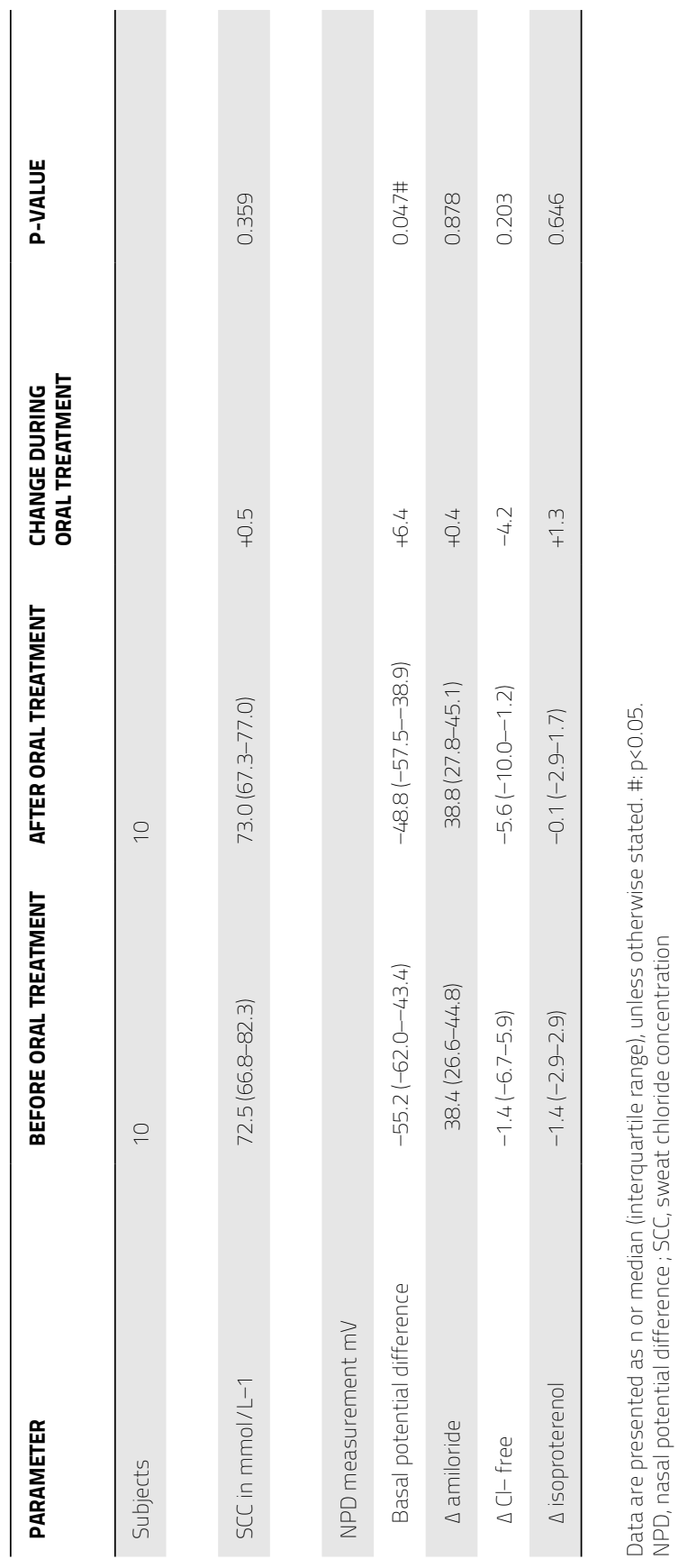


Table 3. SCC and NPD responses to salbutamol aerosol

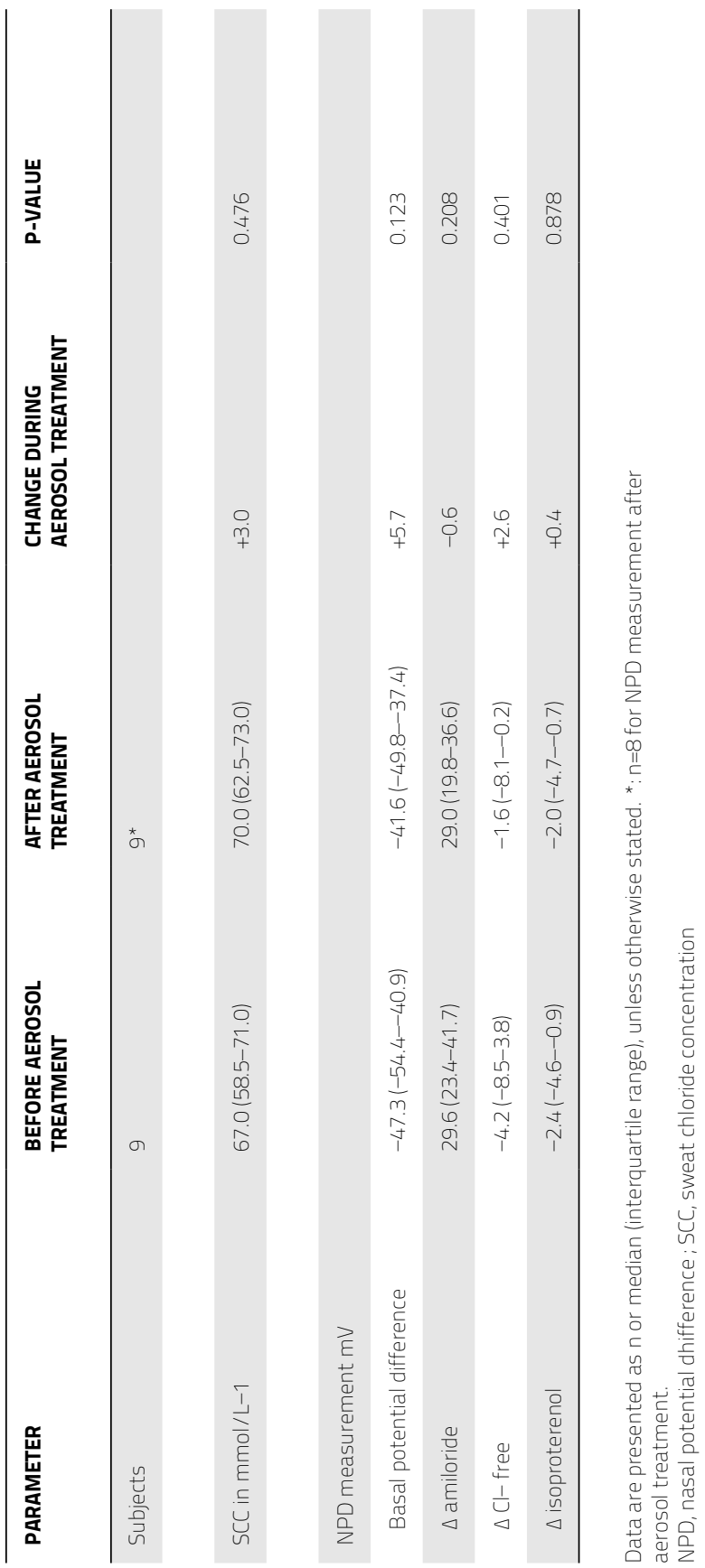


Table 4. Adverse events during treatment with salbutamol

\begin{tabular}{lcc}
\hline ADVERSE EVENT & DURING ORAL TREATMENT & DURING AEROSOL TREATMENT \\
\hline Agitated feeling & 1 & \\
\hline Palpitations & 4 & 1 \\
\hline Cough up more sputum & 2 & 1 \\
\hline Dry mouth & 1 & 1 \\
\hline Tremor & 5 & 1 \\
\hline Headache & 1 & 1 \\
\hline Painful breathing & & \\
\hline
\end{tabular}

Data are presented as $n$ times reported.

phosphorylate and stimulate CFTR activity beyond levels associated with endogenous conditions. We anticipated that NPD could provide the most promising readout for an improvement of CFTR function, as intranasal infusion with the pan-ß-agonist isoproterenol has been shown to further hyperpolarize the nasal epithelium in healthy controls in vivo by an average of $6.9 \mathrm{mV}$ under low luminal chloride conditions, suggesting that CFTR activity in this tissue is rate-limited by endogenous CAMP signaling. ${ }^{31,32}$ Whereas for diagnostic purposes and direct CFTR protein-restoring drugs, the combined change in NPD after addition of zero chloride solution and addition of isoproterenol is most informative, in patients treated with salbutamol we anticipated to find an enhanced response to low chloride but a reduced response to intranasally applied isoproterenol and no difference in the combined response to low chloride plus isoproterenol as both compounds activate endogenous CAMP. Although there was a tendency to an enhanced hyperpolarizing response to zero chloride $(-1.4 \rightarrow-5.6 \mathrm{mV})$ and a decreased response to isoproterenol $(-1.4 \rightarrow-0.1 \mathrm{mV})$ upon oral (but not inhaled) treatment, this difference did not reach statistical significance. The baseline potential difference, which was clearly CF-like in both the A455E and the R117H patients (range -41.6--55.2 mV; Table 2 and Table 3) showed a significant but limited increase towards non-CF baseline potential difference values (by $6.4 \mathrm{mV}$; Table 2) in the orally treated patients, but this change was not paralleled by a reduced response to amiloride, an inhibitor of ENaC (Table 2). Baseline potential appears to be predominated by ENaC-dependent $\mathrm{Na}+$ absorption, which is enhanced in CF and modulated through direct CFTR protein-targeting drugs. ${ }^{33-35}$ This lack of correlation seems to argue against an inhibitory effect of salbutamol treatment on ENaC activity, although the relatively low power of the pilot study and the large variation in potential difference responsiveness to $\mathrm{ENaC}$ does not entirely rule out such an effect. 
The latter interpretation would be in line with the tentative increase in zero chloride response discussed above and the known inhibitory effect of CFTR (through electrogenic or more complex coupling mechanisms) on ENaC activity in the airways. Alternatively, our data do not exclude the possibility that salbutamol inhibits other electrogenic ion transport pathways in the nasal epithelium, such as apical cation channels different from ENaC, e.g. acid-sensitive ion channels or proton channels. ${ }^{36,37}$ Taken together, the outcome of the NPD measurements suggests that oral salbutamol treatment slightly modifies the electrical properties of the nasal CF epithelium towards that of the non-CF nasal epithelium and could therefore be of (limited) benefit for the CF patients

The lack of robust CFTR activation in vivo, in clear contrast with the potent stimulation of organoid swelling by submicromolar levels of salbutamol in vitro (Fig. 2d), is most likely due to the low levels of circulating B2-agonist observed upon in vivo treatment. In line with the findings in the NPD measurements, we only found a detectable CFTR-activating capacity in blood samples that were collected after treatment with oral, but not with inhaled salbutamol. The latter is consistent with a highly limited systemic delivery by ß2agonist inhalation (Fig. 4a). ${ }^{38}$ However, even after treatment with oral salbutamol, CFTRactivating levels in plasma were low (Fig. 4a,b), corresponding with pure salbutamol concentrations in the nanomolar range that can only marginally stimulate organoids (compare Fig. 2c and Fig. 4c). As such, only limited effects in vivo might have been expected, as we observed in only one of the NPD parameters. Although plasma levels are not similar to tissue levels, these data suggest that higher dosages may further improve efficacy of treatment, albeit that systemic side-effects of the treatment may limit the feasibility of increasing the dosage.

The lack of response in SCC is also indicative of a limited treatment response, albeit that this parameter needs to be interpreted with care. SCC is a highly sensitive CFTR function parameter, being capable of distinguishing between pancreas-sufficient and -insufficient groups, and pre-treatment SCC in our patient cohort clearly indicates that these patients have significant residual CFTR activity (Table 3). Furthermore, in G551D patients this biomarker is also highly responsive to CFTR potentiator treatment. ${ }^{10}$ However, isolated sweat glands from subjects also indicate that exogenous $ß$-adrenergic stimuli can only stimulate $\sim 40 \%$ of sweat ducts and later studies confirmed a high constitutive cAMP-dependent activation of CFTR in this tissue. ${ }^{39,40}$ This implies that only a limited window for exogenous B2-agonist stimulation likely exists in vivo in this tissue. The lack of treatment response in SCC we observed in this trial was therefore not completely unexpected considering the high constitutive CFTR activation in this tissue combined with the very low levels of circulating B2-agonists.

Additional clinical studies are required to further validate the effect of long-term treatment with oral B2-agonists on clinical outcome parameters (e.g. percentage predicted forced expiratory volume in $1 \mathrm{~s}$, airway resistance, body mass index, quality of life) in (F patients, as this proof-of-concept study showed a minor but significant impact 
of treatment on the nasal mucosa, but no significant effect on $\mathrm{Cl}$ - transport in the sweat ducts.

As expected, B2-agonists stimulate swelling of organoids in a CFTR mutationdependent manner, based on residual function conferred by the CFTR genotype or by CFTR-modulating drugs (Fig. 2). Most subjects included in the study were compound heterozygous for A455E, and their organoids demonstrate residual CFTR function levels between the values seen with F508del and R117H/F508del compound heterozygotes. ${ }^{20}$ This appears consistent with the SCC parameters measured in this study and with data from the CFTR2 database (www.cftr2.org). Our NPD data (Table 2 and Table 3 and supplemental Table S2 and supplemental Table S3: response to chloride-free and isoproterenol) also showed evidence of residual CFTR function in the A455E patients, with a tendency to increase slightly but not significantly after oral salbutamol treatment. As the VX-770+VX-809-corrected CFTR-F508del function in organoids is higher than the level of residual function associated with CFTR-A455E, B2-agonists may also have added value for F508del homozygous subjects treated with CFTR-repairing drugs. ${ }^{20}$ In this context, cotreatments with B2-agonists may account for some of the pulmonary heterogeneity between patients that is observed in the response to CFTR modulator treatment.9,11 In addition, further stratification for CFTR genotypes with higher residual function (e.g. CFTR-R117H) may also enhance treatment effects with ß2-agonists.

\section{CONCLUSION}

In conclusion, CFTR function measurements in intestinal organoids were used to screen for CFTR-activating drugs and subjects with CFTR variants that respond to these drugs in vitro were selected for in vivo treatment. Oral treatment with salbutamol improved some CF characteristics of the nasal mucosa, but treatment efficacy was likely limited due to ineffective dosage, as apparent from measurements of plasma levels of salbutamol in our organoid-based bioassay. The study supports the concept that intestinal organoids are a valuable tool for selecting drugs and route of administration for CF clinical trials.

\section{ACKNOWLEDGEMENTS}

We thank M.C.J. Olling-de Kok (Dept of Pediatric Pulmonology, Wilhelmina Children's Hospital, University Medical Center, Utrecht, The Netherlands), E.M. NieuwhofStoppelenburg and E.C. van der Wiel (Dept of Pediatric Pulmonology, Erasmus University Medical Center/Sophia Children's Hospital, Rotterdam, The Netherlands), N. Adriaens (Dept of Respiratory Medicine, Academic Medical Center, Amsterdam, The Netherlands), 
and M. Smink (Dept of Pulmonology and Cystic Fibrosis, Haga Teaching Hospital, The Hague, The Netherlands) for providing intestinal biopsies.

Funding: E. Matthes and J.W. Hanrahan were supported by the Canadian Institutes of Health Research. This work was supported by grants from the Dutch Cystic Fibrosis Foundation (NCFS) as part of the HIT-CF programme, the Wilhelmina Children's Hospital (WKZ) Foundation and the Dutch health organisation ZonMW, The Netherlands. Funding information for this article has been deposited with FundRef.

\section{COMPETING INTERESTS}

Disclosures can be found alongside this article at erj.ersjournals.com 


\section{REFERENCES}

1. Riordan JR. CFTR Function and Prospects for Therapy. Annu Rev Biochem. 2008;77(1):701726.

2. Weiler CA, Drumm ML. Genetic Influences on Cystic Fibrosis Lung Disease Severity. Front Pharmacol. 2013;4:40.

3. Vanscoy LL, Blackman SM, Collaco JM, et al. Heritability of Lung Disease Severity in Cystic Fibrosis. Am J Respir Crit Care Med. 2007;175(10):1036-1043.

4. Sosnay PR, Siklosi KR, Van Goor F, et al. Defining the disease liability of variants in the cystic fibrosis transmembrane conductance regulator gene. Nat Genet. 2013;45(10):11601167.

5. Kerem E, Corey M, Kerem B, et al. The Relation between Genotype and Phenotype in Cystic Fibrosis - Analysis of the Most Common Mutation ( $\Delta \mathrm{F} 508$ ). N Engl J Med. 1990;323(22):1517-1522.

6. Castellani $\mathrm{C}$, Cuppens $\mathrm{H}$, Macek $\mathrm{M}$, et al. Consensus on the use and interpretation of cystic fibrosis mutation analysis in clinical practice. J Cyst Fibros. 2008;7(3):179-196.

7. Zielenski J. Genotype and Phenotype in Cystic Fibrosis. Respiration. 2000;67(2):117-133.

8. Van Goor F, Hadida S, Grootenhuis PDJ, et al. Rescue of CF airway epithelial cell function in vitro by a CFTR potentiator, VX-770. Proc Natl Acad Sci. 2009;106(44):18825-18830.

9. Ramsey BW, Davies J, McElvaney NG, et al. A CFTR potentiator in patients with cystic fibrosis and the G551D mutation. N Engl J Med. 2011;365(18):1663-1672.

10. Accurso FJ, Rowe SM, Clancy JP, et al. Effect of VX-770 in persons with cystic fibrosis and the G551D-CFTR mutation. N Engl J Med. 2010;363(21):1991-2003.

11. Wainwright CE, Elborn JS, Ramsey BW, et al. Lumacaftor-Ivacaftor in patients with cystic fibrosis homozygous for Phe508del CFTR. N Engl J Med. 2015:150517100015004.

12. Van Goor F, Hadida S, Grootenhuis PDJ, et al. Correction of the F508del-CFTR protein processing defect in vitro by the investigational drug VX-809. Proc Natl Acad Sci. 2011;108(46):18843-18848.

13. Chan HC, Fong SK, So SC, Chung YW, Wong PY. Stimulation of anion secretion by betaadrenoceptors in the mouse endometrial epithelium. J Physiol. 1997;501 ( Pt 3):517-525.

14. Smith JJ, Welsh MJ. cAMP stimulates bicarbonate secretion across normal, but not cystic fibrosis airway epithelia. J Clin Invest. 1992;89(4):1148-1153.

15. Waldman DB, Gardner JD, Zfass AM, Makhlouf GM. Effects of vasoactive intestinal peptide, secretin, and related peptides on rat colonic transport and adenylate cyclase activity. Gastroenterology. 1977;73(3):518-523.

16. Jung P, Sato T, Merlos-Suárez A, et al. Isolation and in vitro expansion of human colonic stem cells. Nat Med. 2011;17(10):1225-1227.

17. Sato $\mathrm{T}$, Clevers $\mathrm{H}$. Growing self-organizing mini-guts from a single intestinal stem cell: mechanism and applications. Science (80- ). 2013;340(6137):1190-1194.

18. Sato T, Vries RG, Snippert HJ, et al. Single Lgr5 stem cells build crypt-villus structures in vitro without a mesenchymal niche. Nature. 2009;459(7244):262-265.

19. Dekkers JF, van der Ent CK, Beekman JM. Novel opportunities for CFTR-targeting drug development using organoids. Rare Dis. 2013;1(1):e27112.

20. Dekkers JF, Wiegerinck CL, de Jonge HR, et al. A functional CFTR assay using primary cystic fibrosis intestinal organoids. Nat Med. 2013;19(7):939-945. 1

21. Sato $T$, Stange $D E$, Ferrante $M$, et al. Long-term Expansion of Epithelial Organoids From Human Colon, Adenoma, Adenocarcinoma, and Barrett's Epithelium. Gastroenterology. 2011;141(5):1762-1772.

22. de Lau W, Barker N, Low TY, et al. Lgr5 homologues associate with Wnt receptors and mediate R-spondin signalling. Nature. 2011;476(7360):293-297. 
23. Korinek V, Barker N, Morin PJ, et al. Constitutive transcriptional activation by a betacatenin-Tcf complex in APC-/ - colon carcinoma. Science. 1997;275(5307):1784-1787.

24. Bruscia E, Sangiuolo F, Sinibaldi P, Goncz KK, Novelli G, Gruenert DC. Isolation of CF cell lines corrected at DeltaF508-CFTR locus by SFHR-mediated targeting. Gene Ther. 2002;9(11):683-685.

25. Swiatecka-Urban A, Moreau-Marquis S, Maceachran DP, et al. Pseudomonas aeruginosa inhibits endocytic recycling of CFTR in polarized human airway epithelial cells. Am J Physiol Cell Physiol. 2006;290(3):C862-72.

26. Vijftigschild LAW, van der Ent CK, Beekman JM. A novel fluorescent sensor for measurement of CFTR function by flow cytometry. Cytom Part A. 2013;83A(6):576-584.

27. Robert R, Carlile GW, Liao J, et al. Correction of the Delta phe508 cystic fibrosis transmembrane conductance regulator trafficking defect by the bioavailable compound glafenine. Mol Pharmacol. 2010;77(6):922-930.

28. Dekkers R, Vijftigschild LAW, Vonk AM, et al. A bioassay using intestinal organoids to measure CFTR modulators in human plasma. J Cyst Fibros. 2015;14(2):178-181.

29. Walker LC, Venglarik CJ, Aubin G, et al. Relationship between airway ion transport and a mild pulmonary disease mutation in CFTR. Am J Respir Crit Care Med. 1997;155(5):16841689.

30. Naren AP, Cobb B, Li C, et al. A macromolecular complex of 2 adrenergic receptor, CFTR, and ezrin/radixin/moesin-binding phosphoprotein 50 is regulated by PKA. Proc Natl Acad Sci. 2003;100(1):342-346.

31. Knowles MR, Paradiso AM, Boucher RC. In vivo nasal potential difference: techniques and protocols for assessing efficacy of gene transfer in cystic fibrosis. Hum Gene Ther. 1995;6(4):445-455.

32. Boyle MP, Diener-West M, Milgram $L$, et al. A multicenter study of the effect of solution temperature on nasal potential difference measurements. Chest. 2003;124(2):482-489.

33. Rowe SM, Liu B, Hill A, et al. Optimizing nasal potential difference analysis for CFTR modulator development: assessment of ivacaftor in CF subjects with the G551D-CFTR mutation. Boyaka PN, ed. PLoS One. 2013;8(7):e66955.

34. Knowles M, Gatzy J, Boucher R. Relative ion permeability of normal and cystic fibrosis nasal epithelium. J Clin Invest. 1983;71(5):1410-1417.

35. Knowles MR, Stutts MJ, Spock A, Fischer N, Gatzy JT, Boucher RC. Abnormal ion permeation through cystic fibrosis respiratory epithelium. Science. 1983;221(4615):1067-1070.

36. Qadri YJ, Rooj AK, Fuller CM. ENaCs and ASICs as therapeutic targets. Am J Physiol Cell Physiol. 2012;302(7):C943-65.

37. Iovannisci D, Illek B, Fischer H. Function of the HVCN1 proton channel in airway epithelia and a naturally occurring mutation, M91T. J Gen Physiol. 2010;136(1):35-46.

38. Halfhide C, Evans HJ, Couriel J. Inhaled bronchodilators for cystic fibrosis. In: Halfhide C, ed. Cochrane Database of Systematic Reviews. Chichester, UK: John Wiley \& Sons, Ltd; 2005:CD003428.

39. Reddy MM, Quinton PM. PKA mediates constitutive activation of CFTR in human sweat duct. J Membr Biol. 2009;231(2-3):65-78.

40. Reddy MM, Quinton PM. cAMP activation of CF-affected Cl- conductance in both cell membranes of an absorptive epithelium. J Membr Biol. 1992;130(1):49-62. 


\section{SUPPLEMENTALS}

Supplemental Table S1. GPCR compound library

\begin{tabular}{|c|c|}
\hline ADL5859 HCl & $850173-95-4$ \\
\hline Granisetron $\mathrm{HCl}$ & 107007-99-8 \\
\hline SB939 & $929016-96-6$ \\
\hline Carvedilol & 72956-09-3 \\
\hline Ketanserin (Vulketan Gel) & $74050-98-9$ \\
\hline Domperidone (Motilium) & 57808-66-9 \\
\hline Maprotiline hydrochloride & $10347-81-6$ \\
\hline Dehydroepiandrosterone(DHEA) & $53-43-0$ \\
\hline JTC-801 & $244218-51-7$ \\
\hline Bisoprolol & $104344-23-2$ \\
\hline Naftopidil Dihydrochloride & 57149-08-3 \\
\hline AM-1241 & $444912-48-5$ \\
\hline Dapoxetine hydrochloride (Priligy) & 129938-20-1 \\
\hline Loperamide hydrochloride & $34552-83-5$ \\
\hline Naphazoline hydrochloride (Naphcon) & $550-99-2$ \\
\hline WAY-100635 & $162760-96-5$ \\
\hline Fingolimod FTY720 & $162359-56-0$ \\
\hline Agomelatine & $138112-76-2$ \\
\hline Alfuzosin hydrochloride(Uroxatral) & 81403-68-1 \\
\hline Dapagliflozin & $461432-26-8$ \\
\hline Enalapril maleate (Vasotec) & $76095-16-4$ \\
\hline Amfebutamone (Bupropion) & $31677-93-7$ \\
\hline Olanzapine (Zyprexa) & 132539-06-1 \\
\hline Epinephrine bitartrate (Adrenalinium) & $51-42-3$ \\
\hline LY310762 & $192927-92-7$ \\
\hline LY404039 & $635318-11-5$ \\
\hline Dimebon (Latrepirdine) & $97657-92-6$ \\
\hline Nebivolol (Bystolic) & $152520-56-4$ \\
\hline Benserazide & $14919-77-8$ \\
\hline Oxymetazoline hydrochloride & $2315-02-8$ \\
\hline
\end{tabular}




\begin{tabular}{|c|c|}
\hline Dopamine hydrochloride (Inotropin) & $62-31-7$ \\
\hline BRL-15572 & $193611-72-2$ \\
\hline Amisulpride & $71675-85-9$ \\
\hline Sumatriptan succinate & $103628-48-4$ \\
\hline Silodosin (Rapaflo) & $160970-54-7$ \\
\hline Chlorpromazine (Sonazine) & 69-09-0 \\
\hline Racecadotril (Acetorphan) & $81110-73-8$ \\
\hline Ritodrine hydrochloride (Yutopar) & $23239-51-2$ \\
\hline ADX-47273 & $851881-60-2$ \\
\hline Asenapine & $85650-56-2$ \\
\hline Tianeptine sodium & $30123-17-2$ \\
\hline Risperidone (Risperdal) & $106266-06-2$ \\
\hline Clonidine hydrochloride (Catapres) & $4205-91-8$ \\
\hline Salbutamol sulfate (Albuterol) & $51022-70-9$ \\
\hline Clomipramine hydrochloride (Anafranil) & $17321-77-6$ \\
\hline BMY 7378 & $21102-95-4$ \\
\hline Doxazosin mesylate & $77883-43-3$ \\
\hline Venlafaxine & $99300-78-4$ \\
\hline Quetiapine fumarate (Seroquel) & $111974-72-2$ \\
\hline
\end{tabular}


Supplemental Table S2. SCC and NPD response to oral salbutamol per subject included in the pilot study.

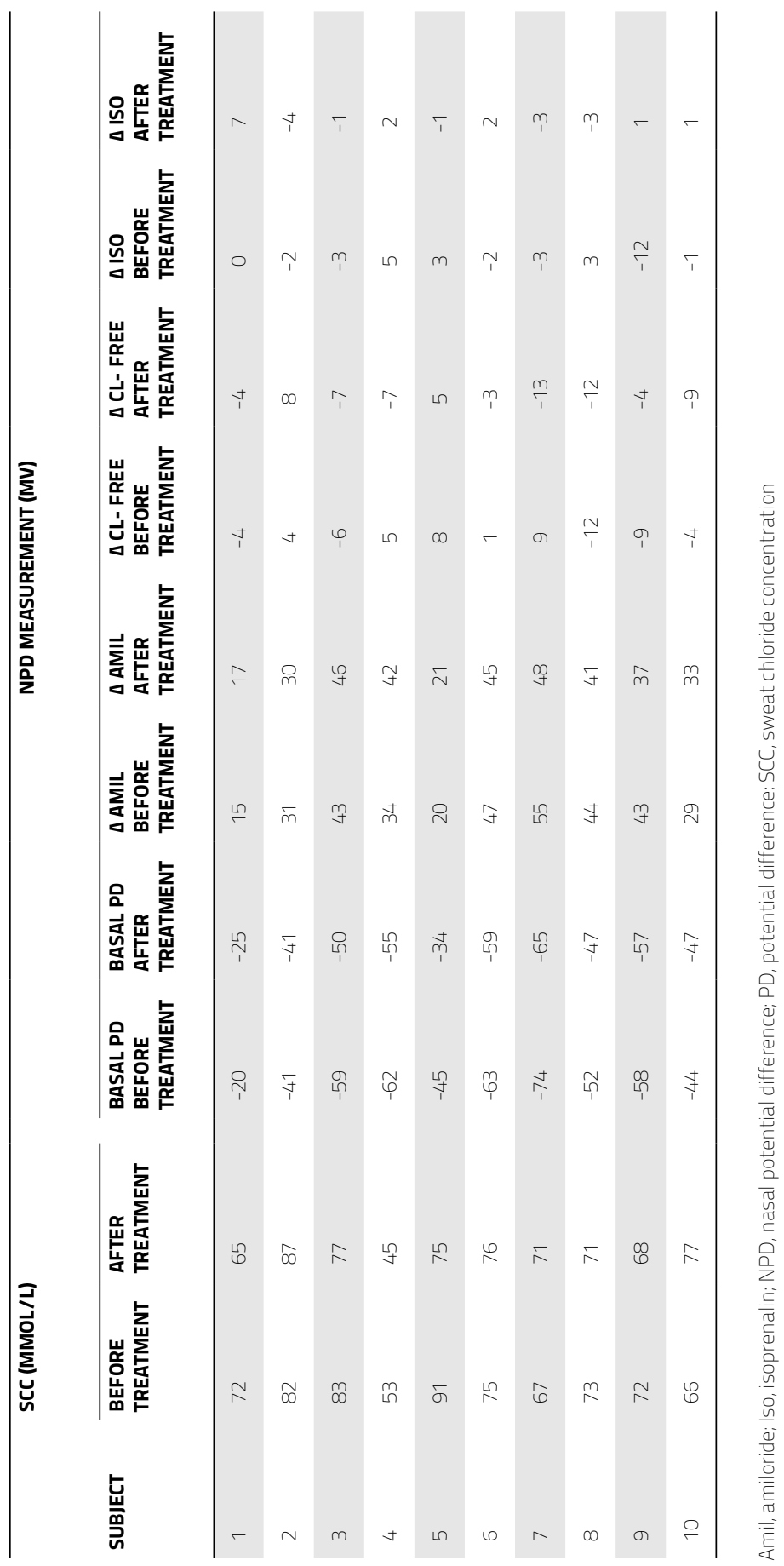


Supplemental Table S3. SCC and NPD response to salbutamol aerosol per subject included in the pilot study. 


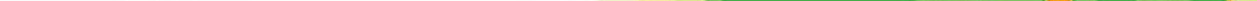




\section{CHAPTER 3}

\section{CLINICAL EFFECTS OF THE THREE CFTR POTENTIATOR TREATMENTS CURCUMIN, GENISTEIN AND IVACAFTOR IN PATIENTS WITH THE CFTR-S1251N GATING MUTATION}

Gitte Berkers, Renske van der Meer, Peter van Mourik, Annelotte M. Vonk, Evelien Kruisselbrink, Sylvia W.F. Suen, Harry G.M. Heijerman, Christof J. Majoor, Gerard H. Koppelman, Jolt Roukema, Hettie M. Janssens, Yolanda B. de Rijke, E. Marleen Kemper, Jeffrey M. Beekman, Cornelis K. van der Ent, Hugo R. de Jonge 


\section{ABSTRACT}

Background: The natural food supplements curcumin and genistein, and the drug ivacaftor were found effective as cystic fibrosis transmembrane conductance regulator potentiators in the organoids of individuals carrying an $51251 \mathrm{~N}$ gating mutation, possibly in a synergistic fashion. Based on these in vitro findings, we evaluated the clinical efficacy of a treatment with curcumin, genistein and ivacaftor, in different combinations.

Methods: In three multi-centre trials people with cystic fibrosis carrying the S1251N mutation were treated for 8 weeks with curcumin + genistein, ivacaftor and ivacaftor + genistein. We evaluated change in lung function, sweat chloride concentration, cystic fibrosis questionnaire-revised, body mass index and faecal elastase to determine the clinical effect. We evaluated the pharmacokinetic properties of the compounds by evaluating the concentration in plasma collected after treatment and the effect of the same plasma on the intestinal organoids.

Results: A clear clinical effect of treatment with ivacaftor was observed, evidenced by a significant improvement in clinical parameters. In contrast we observed no clear clinical effect of curcumin and/or genistein, except for a small but significant reduction in sweat chloride and airway resistance. Plasma concentrations of the food supplements were low, as was the response of the organoids to this plasma.

Conclusions: We observed a clear clinical effect of treatment with ivacaftor, which is in line with the high responsiveness of the intestinal organoids to this drug. No clear clinical effect was observed of the treatment with curcumin and/or genistein, the low plasma concentration of these compounds emphasizes that pharmacokinetic properties of a compound have to be considered when in vitro experiments are performed. 


\section{INTRODUCTION}

Cystic Fibrosis (CF) is an autosomal recessive disease that affects over 70.000 patients worldwide. ${ }^{1}$ In the last decade new treatments that enhance the function of the mutated cystic fibrosis transmembrane conductance regulator (CFTR) protein have been developed and introduced in the care for patients with CF. Currently, these treatments are available for only part of the patients with CF, depending on the combination of (the more than 2000 known) CFTR variants. ${ }^{2}$ Clinical studies on the effect of these CFTR modulators show impressive improvement in clinical parameters, but indicate that CFTR function after treatment appears not yet on the level of healthy controls. ${ }^{3-9}$ Another limitation of the currently available CFTR modulators is their lack of accessibility, which highlights the importance of exploring new therapies that can increase in vivo CFTR function, using interventions that are easily accessible to people.

In previous studies, we showed that we can generate intestinal organoids by isolating stem cells from rectal biopsies and expanding them in a 3D culture condition. ${ }^{10,11}$ Adding the adenylyl cyclase activator forskolin to these cultures induces CFTR dependent swelling of organoids (forskolin induced swelling (FIS)) which can be used to study the residual CFTR function of an individual with CF, and the response to CFTR modulating drugs. ${ }^{11-14}$ This makes this assay an ideal drug-screening platform to evaluate the effect of (combinations of) drugs on the function of the CFTR protein, also for individuals with specific mutations.

Using the intestinal organoid model to evaluate the in vitro effect of different compounds on CFTR function showed that the natural food supplements curcumin and genistein, and the potentiator ivacaftor (VX-770) were effective as CFTR potentiators in organoids of patients with a p.Ser1251Asn (S1251N) gating mutation. ${ }^{15}$ These in vitro experiments also showed a synergistic effect of these compounds, especially when genistein and ivacaftor were combined; the organoid response upon combined addition resulted in twice the organoid response compared to the sum of the responses upon addition of the single compounds. Earlier in vitro studies also showed that both curcumin and genistein can improve the gating of the CFTR protein through an ATP-independent and ATP-dependent mechanism, respectively. ${ }^{16-18}$ The synergistic effect of curcumin and genistein on another CFTR gating mutant, G551D, has been previously described in two in vitro patch-clamp studies. ${ }^{19,20}$ However whether the synergistic effect of these food supplements can also be observed in people with CF remains unknown. ${ }^{19-21}$ In contrast, ivacaftor has not only shown positive effects on CFTR gating in in vitro studies, but clinical trials have clearly confirmed its efficacy in vivo, mainly in patients with a CFTR gating mutation. ${ }^{3-5,22}$ Worldwide S1251N is a relatively rare CFTR mutation, however, it is the most common gating mutation in The Netherlands. ${ }^{23}$ Previous clinical trials showed that ivacaftor treatment of patients with the $51251 \mathrm{~N}$ mutation resulted in a clear clinical effect, comparable to its effect in patients with the more common G551D gating mutation. ${ }^{3,4}$ 
Here we asked whether the (combinations of) compounds that potentiated CFTR function in intestinal organoids would also be efficacious in people carrying the S1251N mutation. Therefore, we conducted three clinical trials in which we treated individuals with S1251N with (a combination of) curcumin, genistein and ivacaftor. As a secondary aim we investigated the plasma levels of curcumin, genistein and ivacaftor achieved in these patients and investigated the effect of plasma that was collected after treatment, in the organoid assay.

\section{METHODS}

\section{Design}

We performed three multicenter clinical trials with different (combinations of) CFTR potentiators. Study 1 (NTR4585) was an eight-weeks open label intervention study with a combination of curcumin (AOV 811 curcuma longa) and genistein (AOV 805 genistein). Both compounds were produced by the company AOV (part of Atrium Innovations INC), information on the formulation specification and quality can be found in supplemental Table S1. Dosage schemes of curcumin and genistein were based on doses that were used in previous clinical trials described in literature. Curcumin dose was between 102.9 and $138.5 \mathrm{mg} / \mathrm{Kg} /$ day and genistein dose was between 3.3 and $5.0 \mathrm{mg} / \mathrm{Kg} /$ day in $3-4$ doses (supplemental Fig. S1). Study 2, (NTR4873) was an eight-weeks observational study in patients that started with ivacaftor at the time that it was introduced in The Netherlands(supplemental Fig. S2). All patients received ivacaftor in a dose of $150 \mathrm{mg}$, twice daily. Study 3 (NTR6515) was a randomized double-blind placebo controlled crossover study with eight weeks of genistein treatment (AOV 805 genistein, between 5.0 and $10.0 \mathrm{mg} / \mathrm{kg} /$ day) eight weeks of treatment with matched placebo and a 4 weeks washout period, in patients already receiving ivacaftor (supplemental Fig. S3). The placebo capsules were produced by the Erasmus MC hospital pharmacy ("Apotheek A15") which is certified according to "good manufacturing practice" (GMP) standards.

\section{Subjects}

Included patients had at least one S1251N mutation, were at least 6 years old and already had a rectal biopsy to produce an organoid. Patients were not included in a study if they were already using another CFTR modulator (besides ivacaftor in study 3). In study 1 and 3, patients could not be included if there were any contra-indications for the use of curcumin and/or genistein or if they had an exacerbation needing intravenous therapy in the four weeks prior to the start of the study. The study protocols were reviewed and approved by the medical ethics committee of the University Medical Center Utrecht. All participants (and/or their legal representatives) provided written informed consent. 


\section{Clinical outcomes}

In all 3 studies we evaluated the clinical effect of the treatment by measuring change in: percentage of predicted forced expiratory volume in one second (ppFEV1, measured according to ATS-ERS standards and converted to percentage of predicted using reference values of Koopman et al and Quanjer et al. ${ }^{24-26}$, airway resistance, sweat chloride concentration (SCC, sweat collected using a Macroduct ${ }^{\circledR}$ system and analysis performed according to the most recent version of the standard operating procedure of the European Cystic Fibrosis Society-Clinical Trial Network), quality of life examined by the cystic fibrosis questionnaire - revised (CFQ-r), fecal elastase concentration and body mass index (BMI). To monitor safety, patients were asked to keep a diary in which they had to report any possible side effects. Both the relation of these adverse events ( $A E^{\prime}$ 's) to the study drug and the severity of the AE's (a 5 point scale from mild to fatal) was scored by a medical doctor at the site.

\section{Blood collection and storage}

Before and after each treatment period a blood sample was collected which was used to measure the plasma concentration of the compound and also to evaluate the effect of the plasma in the intestinal organoid model. At the end of each treatment period the blood collection was performed at the expected T-max of the treatment (3h for curcumin + genistein, 4h for ivacaftor and ivacaftor + genistein). Blood was collected in a Vacuette ${ }^{\circledR}$ heparin tube and centrifuged during $20 \mathrm{~min}$ at $450 \mathrm{~g}$ at $4^{\circ} \mathrm{C}$ to collect the plasma, which was immediately stored at $-80^{\circ} \mathrm{C}$. Methods of measuring plasma concentrations of the three compounds as well as their metabolites can be found in supplemental Table S2.

\section{Organoid measurements}

Organoids were cultured according to previously described protocols. ${ }^{11}$ Organoid swelling was measured as biological duplicates and FIS experiments were repeated at three independent culture time points. To evaluate FIS, calcein green labeled organoids were monitored by a Zeiss LSM800 live cell confocal microscope $\left(37^{\circ} \mathrm{C}, 5 \% \mathrm{CO}\right)$ for 60 minutes with 10 minute intervals. Zen Image analysis software (Zeiss) was used to quantify the total organoid area $\left(\mu \mathrm{m}^{2}\right)$ per well for each time point after which relative swelling increase over time was normalized to time point zero ( $\mathrm{t}=0$ min baseline of $100 \%$. Response was defined by the area under the curve.

The response to the three potentiator treatments was evaluated in the intestinal organoids of patients included in the trials. The organoids were stimulated with a combination of $10 \mu \mathrm{M}$ genistein (Sigma) plus $50 \mu \mathrm{M}$ curcumin (Sigma), with $3 \mu \mathrm{M} \mathrm{VX}$ 770/ivacaftor (Selleck Chemicals LLC) and with a combination of $3 \mu \mathrm{M}$ VX-770/ivacaftor (Selleck Chemicals LLC) plus $10 \mu \mathrm{M}$ genistein (Sigma)) together with $0.128 \mu \mathrm{M}$ forskolin. The FIS observed after adding the three (combinations of) compounds was normalized to 
the mean FIS in response to dimethylsulfoxide (DMSO).

Organoid responses to plasma that was collected after treatment was evaluated together with forskolin (1.5\% plasma and $0.128 \mu \mathrm{M}$ forskolin for curcumin + genistein and ivacaftor treatment, $1 \%$ plasma and $0.320 \mu \mathrm{M}$ forskolin for ivacaftor + genistein treatment) and normalized to the mean FIS in response to blank plasma under the same conditions.

\section{Statistical analysis}

Because of the limited number of patients with the S1251N mutation in the Netherlands, we were not able to power the study for small clinical effects. No formal power calculation was performed and a sample size of 15-20 patients per study was estimated based on the number of patients with the S1251N mutation numbers. Descriptive statistics were used to describe the study population included in the three trials. Depending on the nature and distribution of the characteristics we calculated proportions, means (including standard deviation (sd)) and medians (including interquartile range (IQR)). The effect of a treatment was evaluated by comparing the mean or median value of an outcome parameter in the study population before the start of a treatment with the mean or median value in the study population after eight weeks of treatment. For this comparison a paired samples t-test or Wilcoxon signed rank test was used, depending on the distribution of the values. A p-value $<0.05$ was considered to indicate a statistically significant change. Analysis were performed using IBM SPSS Statistics version 22.

\section{RESULTS}

\section{Participants}

A total of 13,16 and 14 patients participated in study 1,2 and 3 respectively. There were 13 patients who participated in both study 1 and 2 , and 9 patients participated in all three studies. There were no dropouts during the studies, consort diagrams for each study can be found in supplemental Fig. S4. The baseline characteristics of the included patients are shown in Table 1. This table not only shows that most patients carried the F508del mutation on the second allele, but also indicates that for most parameters the baseline values were already in the normal range at the start of study 3.

\section{Clinical effect}

Overall, treatment with ivacaftor resulted in a clear clinical effect showing significant improvement in virtually all clinical outcome parameters tested. For study 1 (curcumin + genistein) we observed no change in ppFEV1 but in study 2 (ivacaftor) we observed a statistically significant increase of median ppFEV1 of $9.5 \%$ compared to baseline. 
Treatment of patients with genistein on top of ivacaftor treatment (study 3) did not result in any further increase of median ppFEV1. An overview of the changes in ppFEV1 of individual patients is provided in supplemental Fig. S5. Changes in the other outcome parameters were in line with these findings in ppFEV1, except for a small but statistically significant change of SCC and airway resistance upon curcumin + genistein treatment (Table 2). Analysis of the collected plasma showed that for the majority of patients (10 out of 13) curcumin was not detectable. Genistein showed median plasma concentrations of 3 and $14 \mu \mathrm{g} / \mathrm{L}$ (i.e. 0.01 and $0.05 \mu \mathrm{M}$ ) in study 1 and 3 respectively, and median ivacaftor concentrations were 1942 and $1160 \mu \mathrm{g} / \mathrm{L}$ (i.e. 4.95 and $2.96 \mu \mathrm{M}$ ) in study 2 and 3 respectively. Metabolites of curcumin and genistein after treatment in study 1 (shown in supplemental Table S3) were in the micromolar range.

\section{Organoid responses}

To study the CFTR potentiating effect of the test compounds at plasma concentrations that were reached during treatment in vivo, we applied an organoid-based bioassay developed previously for measuring functional levels of the CFTR potentiator ivacaftor in human plasma and compared the organoid swelling responses to plasma collected after treatment with the responses to purified compounds (Fig. 1). ${ }^{27}$ In line with previous observations we found that micromolar concentrations of curcumin + genistein, ivacaftor and ivacaftor + genistein added in vitro to S1251 N intestinal organoids were all capable of inducing forskolin-activated, CFTR-mediated organoid swelling. ${ }^{15}$ In contrast, a clear gain in forskolin-induced organoid swelling was detected only with plasma samples collected after patient treatment with ivacaftor (study 2) but not when stimulated with plasma samples obtained after combined treatment with the food supplements genistein and curcumin or addition of genistein to ivacaftor treatment (study 1 and 3; Fig. 1).

\section{Adverse events}

The AE's that were reported by more than one patient are shown in Table 3. In all three studies the majority of patients reported at least one AE. The AE's that occurred during treatment with curcumin + genistein and were possibly or probably related to the study drug were headache and gastro-intestinal symptoms. During treatment with ivacaftor the reported AE's that were possibly or probably related to the study drug were coughing up more sputum, headache and gastro-intestinal symptoms. During study 1 and 3 there were no serious adverse events (SAE's), during study 2 there were 4 SAE's reported, of which none was possibly or probably related to the study drug. 
Figure 1. Organoid response to pure drug and plasma collected after treatment with drug

\section{Organoid response $(n=3)$}

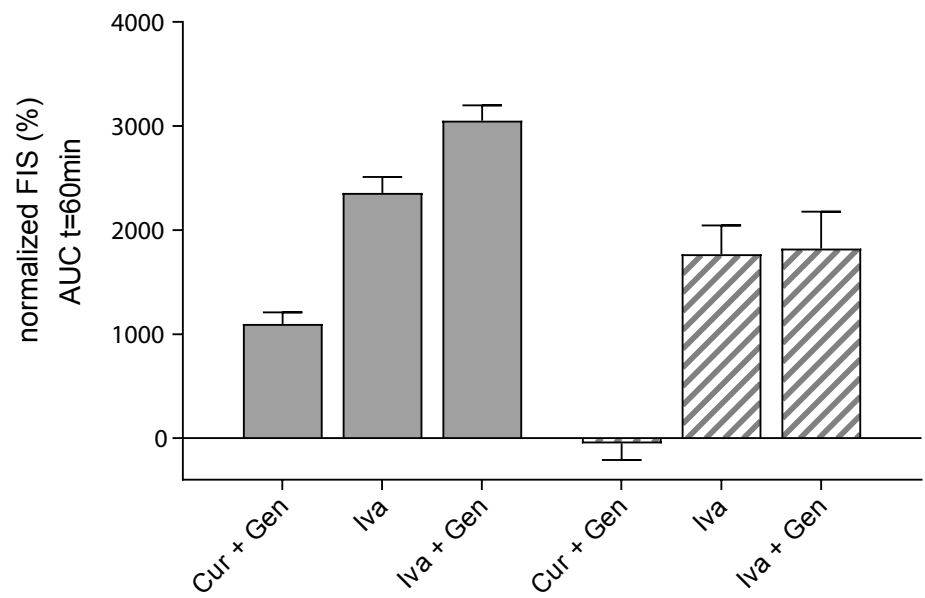

$\square$ Pure drug

Qu Plasma

Organoid swelling was measured as biological duplicates and FIS experiments were repeated at three independent culture time points. (Mean \pm SD of three culture points).

Organoid response to the pure drug (left): Area under the curve (AUC) of swelling of organoids from patients included in the trials after measuring for 60 minutes. Responses after adding a combination of $10 \mu \mathrm{M}$ genistein plus $50 \mu \mathrm{M}$ curcumin, $3 \mu \mathrm{M} V X-770 /$ ivacaftor and a combination of $3 \mu \mathrm{MVX}$-770/ivacaftor plus $10 \mu \mathrm{M}$ genistein all together with $0.128 \mu \mathrm{M}$ forskolin. Responses are normalized to the mean organoid response to DMSO with $0.128 \mu \mathrm{M}$ forskolin.

Organoid responses to plasma collected after treatment (right): AUC of swelling of organoids with a F508del/ S1251N mutation after measuring for 60 minutes. Responses after adding 1.5\% plasma together with $0.128 \mu \mathrm{M}$ forskolin (for plasma collected after treatment with curcumin + genistein and ivacaftor) and 1\% plasma together with $0.320 \mu \mathrm{M}$ forskolin (for plasma collected after treatment with ivacaftor + genistein). Responses are normalized to the mean organoid response to blank plasma in combination with 0.128 $\mu \mathrm{M}$ forskolin (for curcumin + genistein and ivacaftor) and $0.320 \mu \mathrm{M}$ forskolin (for ivacaftor + genistein). 
Table 1. Baseline characteristics of patients

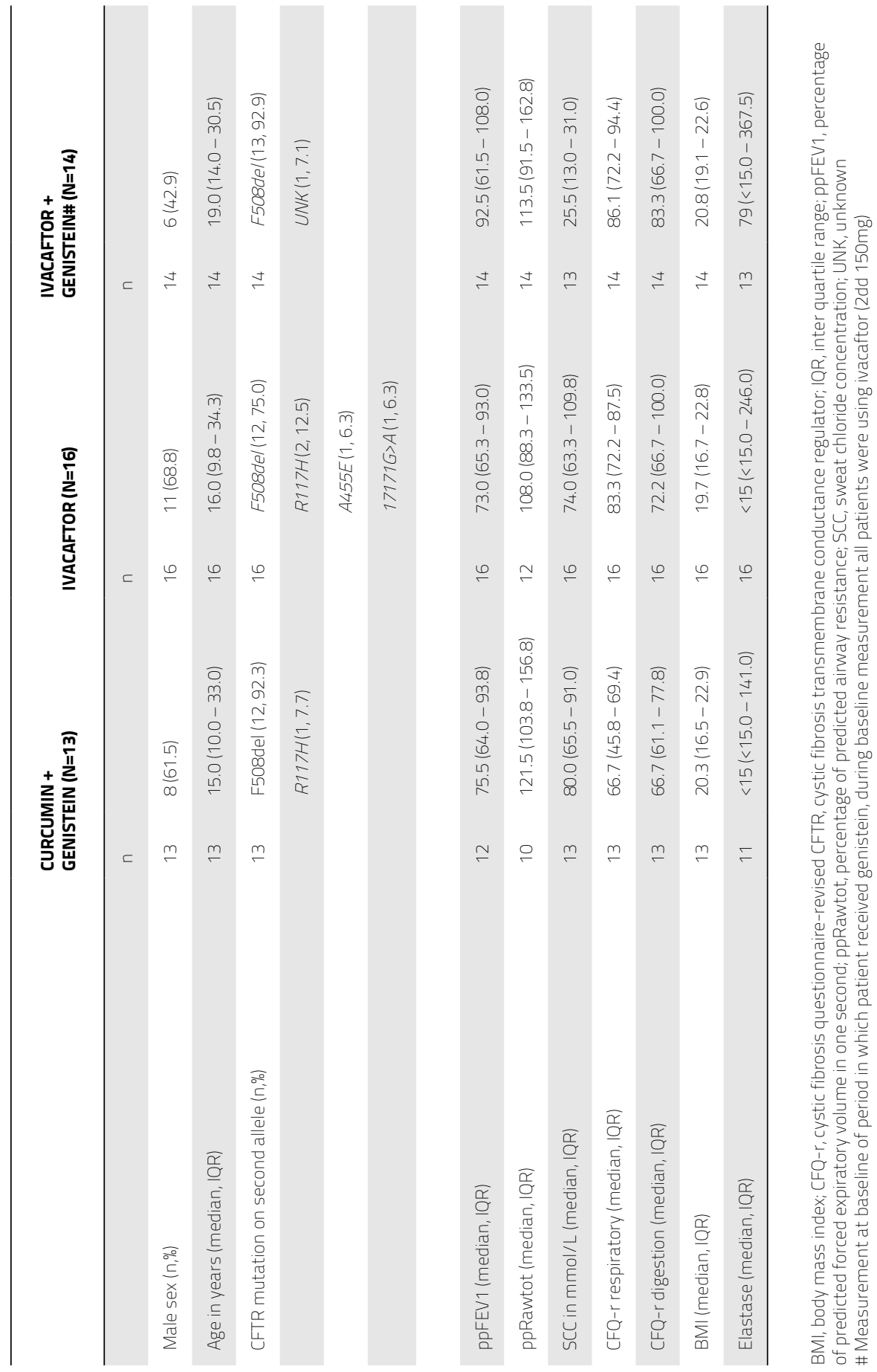




\section{DISCUSSION}

In this study the effect of oral administration of three compounds with CFTR potentiator activity in intestinal organoids was evaluated in people carrying the S1251N CFTR gating mutation. The three clinical trials performed are among the first drug trials that were initiated on the basis of their CFTR stimulating effect in the intestinal organoid model. Although not all trials were performed in a double blinded setting, they all had a comparable design which included an eight-weeks treatment period and similar outcome parameters.

We observed a clear clinical effect of treatment with ivacaftor alone (study 2), with a significant improvement in $\mathrm{pPFEV}_{1}, \mathrm{SCC}, \mathrm{CFQ}-\mathrm{r}$ and BMI values. The observed clinical effectiveness of ivacaftor therapy was comparable to the outcome of a previous trial on the effect of ivacaftor in patients with a non-G551D mutation. ${ }^{4}$ In contrast, combination therapy with the natural food supplements curcumin and genistein (study 1) or supplementation of ivacaftor with genistein (study 3) failed to show a statistically significant clinical effect. Despite the dosages of the supplements approached the upper limits used in previous clinical trials (supplemental Table S3), the plasma concentrations of the food supplements during treatment were in the submicromolar range, which is far below the concentration that was effective in the in vitro organoid swelling experiments (3-100 $\mu \mathrm{M}$ genistein; 25-200 $\mu \mathrm{M}$ curcumin) ${ }^{15}$. The plasma concentrations of ivacaftor were in line with the known pharmacokinetic properties of this compound and reached concentration (3-5 $\mu \mathrm{M})$ corresponding with maximal swelling of the S1251N organoids in vitro. ${ }^{15,28}$

In comparison with previous pharmacokinetic studies in which comparable doses of curcumin and genistein were taken orally (supplemental Table S3) the plasma concentrations of genistein and curcumin measured in our study are remarkably low. However, when we also take the concentration of metabolites and conjugates into account, both curcumin and genistein reached concentrations that were very similar to the values reported in the literature (405 and $955 \mu \mathrm{g} / \mathrm{L}$ respectively). This comparison suggests that the previous studies have included metabolites and conjugates of curcumin and genistein in their analysis as well. Importantly, the results of our clinical trials and the lack of organoid response to the plasma of treated patients suggest that these metabolites, unlike the original compound, are unable to stimulate CFTR as we see no clear functional effect in vivo and in vitro, with the possible exception of the sweat glands. The modest but significant reduction in sweat chloride levels observed in study 1 (Table 2) suggests that curcumin and/or genistein may accumulate in the sweat duct over time and might reach high enough local concentrations to stimulate S1251N CFTR in a subset of patients.

Most plausibly, the low plasma concentrations of genistein and curcumin reached in the 
Table 2. Effects of potentiator treatment

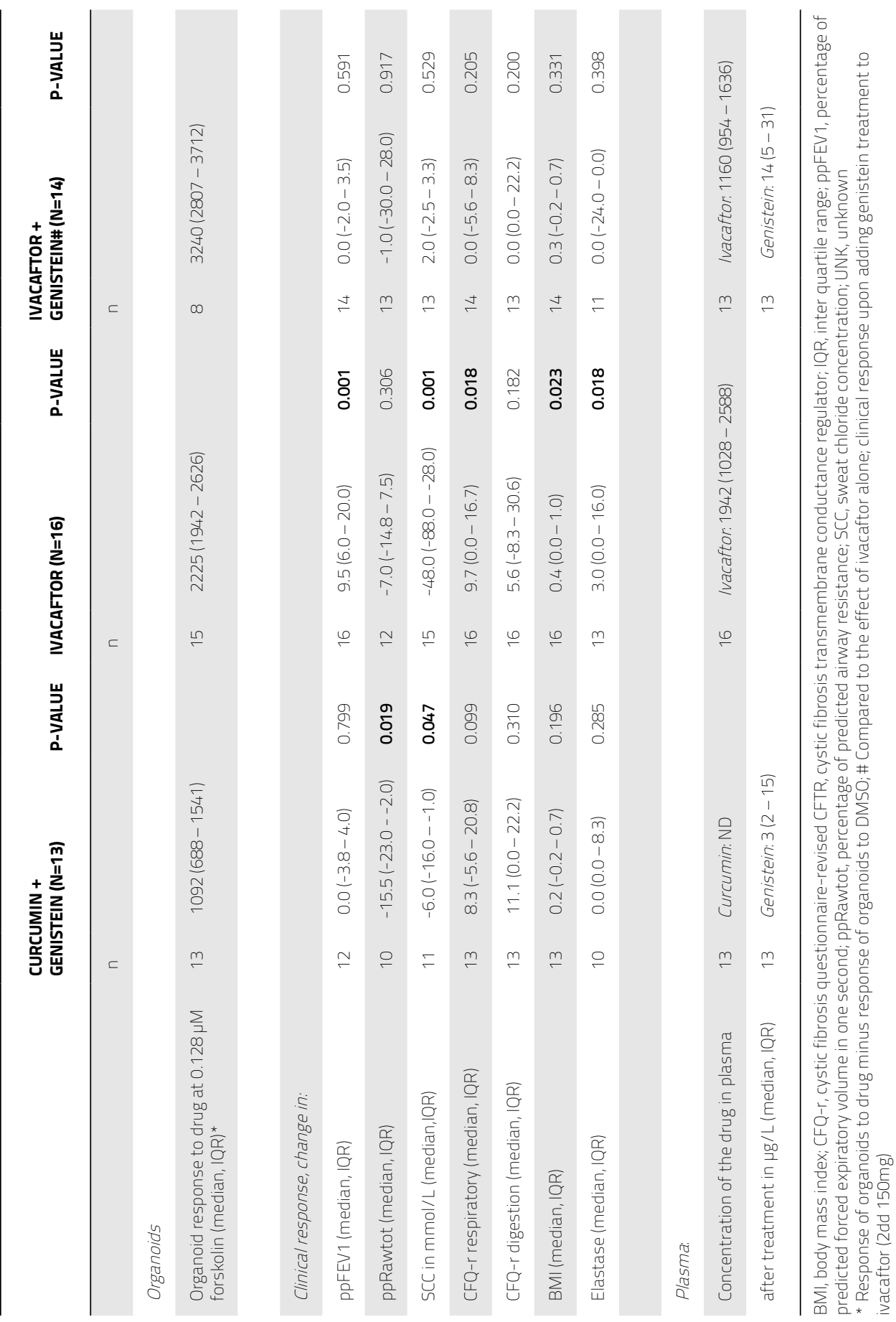


Table 3. Adverse events

CURCUMIN +

GENISTEIN

IVACAFTOR

( $N=13)$

( $N=16)$

IVACAFTOR +

GENISTEIN\#

( $N=14$ )

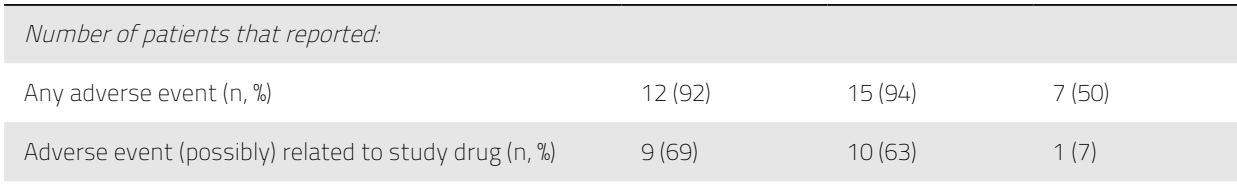

The following adverse events were reported ${ }^{*}(n, \%)$

Bloating

Common cold

Cough

Cough up more sputum

Decreased appetite

Diarrhea

\section{Flatulence}

Headache

Malaise

Nausea

Night sweats

Sinus complaints

Skin rash

Sore eyes

Sore nose

Sore throat

Sputum less viscous

Stomach ache

Throwing up

Vertigo

Yellow stool
$1(6)$

1 (6)

$1(8)$

1 (8)

$7(44)$

$1(6)$

$1(8)$

$1(6)$

$1(7)$

$3(23)$

4(31)

$5(31)$

$1(7)$

1 (8)

$1(8)$

$3(19)$

1 (8)

$-\quad 1(6)$

$\begin{array}{lll}- & - & 1(7)\end{array}$

$1(6)$

$-\quad 1(6)$

$-\quad 1(6)$

$-\quad 1(6)$

2 (15)

4 (25)

$1(7)$

$1(8)$

$2(13)$

$1(8)$

$4(31)$

*Only adverse events that were (possibly) related to the study drug and were reported by more than one patient are shown \#Events upon adding genistein treatment to ivacaftor (2dd 150mg) 
circulation, and the apparent lack of CFTR potentiation by their metabolites underlie the lack of clinical efficacy observed. Moreover, in case of study 3 , all clinical parameters were already drastically improved by the ivacaftor treatment and were for some already within the normal range (Table 1 and Table 2). This makes it difficult to detect a potential further clinical improvement by the additional administration of genistein.

Poor absorption of the food supplements from the intestinal tract, in combination with their fast metabolism in the liver could well account for the low plasma levels found in our clinical trials. Here it should be noted that, among the various CFTR-expressing epithelia, intestinal epithelial cells are exposed at their apical border to much higher concentrations of the food supplements passing the gut and therefore would have a better chance of functional repair by these CFTR potentiators. Unfortunately the most direct test to verify this assumption, i.e. CFTR intestinal current measurements (ICM) in rectal biopsies before and after treatment, was not included in our studies. ${ }^{29}$ However our studies did allow an indirect assessment of CF-related gastro intestinal dysfunction in the S1251N patients on the basis of CFQ-r, BMI and fecal elastase tests. The outcome of these tests did not reveal signs of a significant improvement following an eight weeks treatment with the food supplements.

Recent attempts in healthy volunteers to improve oral bioavailability of curcumin by its dispersion with colloidal nanoparticles (named theracurmin) resulted in a $\sim 27$-fold higher area under the blood concentration-time curve (area under the curve, AUC) in comparison with curcumin powder. ${ }^{30}$ However the serum levels reached remained within the submicromolar range, i.e. below the level needed for effective CFTR potentiation. ${ }^{31}$ Intravenous administration of food supplements could in principle bypass this hurdle, but chronic treatment would be strenuous for the patient and would no longer meet the "Generally recognized as safe" (GRAS) FDA designation of the food supplements.

Because there are a limited number of patients with an S1251N mutation in the Netherlands, we could not include more patients in the trials. As a result the trials were underpowered to detect small changes in clinical parameters, nevertheless the results of treatment with ivacaftor show that a clear clinical effect could be identified.

\section{CONCLUSION}

In conclusion, we evaluated the effect of three CFTR modulator treatments, which were found effective in intestinal organoids of patients with an S1251N mutation. We observed a clear improvement in multiple clinical parameters upon treatment with ivacaftor, which is in line with the clear functional response of intestinal organoids to this drug. This further supports that intestinal organoids can be used as a valuable tool for in vitro drug screening and selection of therapies. The lack of a clear and statistically 
significant improvement in the clinical parameters upon treatment with the natural food supplements curcumin and genistein most plausibly results from the low plasma concentration of these compounds. This highlights the importance of implementation of the pharmacokinetic properties of a compound when performing in vitro experiments. We can account for these pharmacokinetic properties by measuring the swelling response of organoids generated from rectal biopsies to plasma samples of the patient who is using the drug.

\section{ACKNOWLEDGEMENTS}

We thank all patients who gave informed consent for participating in the clinical trials; all the members of the research teams that contributed to this work, especially E.M. Nieuwhof-Stoppelenburg, E.C. Kooij - van der Wiel (Department of Pediatric Pulmonology, Erasmus Medical Center/Sophia Children's Hospital, Rotterdam, Netherlands), N. Adriaens and P.F.M. Mau Asam (department of Respiratory Medicine Amsterdam University Medical Centers, Amsterdam, Netherlands), M. Smink, and I. Paalvast-Schouten (Haga Teaching Hospital, The Hague, Netherlands), S. Heida-Michel, M. Geerdink, I. Janse-Seip, H. van Panhuis, M.C.J. Olling-de Kok (Department of Pediatric Pulmonology, Wilhelmina Children's Hospital, University Medical Center Utrecht, Utrecht University, Utrecht, Netherlands), E. Wilms (Apotheek Haagse Ziekenhuizen, Haga Teaching Hospital, The Hague, Netherlands) E.M. van Maarseveen (Clinical Pharmacy, University Medical Center Utrecht, Utrecht University, Utrecht, Netherlands); and MCO health BV for donating "AOV 811 curcuma longa" and "AOV 805 genistein"

Funding: This work was supported by grants of the Dutch Cystic Fibrosis Foundation (NCFS) as part of the HIT-CF program and the Dutch Health Organization ZonMw, Netherlands (PTO project 95104041).

\section{COMPETING INTERESTS}

J.M.B. and C.K.v.d.E are inventors on a patent application related to these findings.

GHK reports research funding from the Lung Foundation of the Netherlands, GSK, Ubbo Emmius Foundation, Vertex, TEVA the Netherlands, TETRI Foundation, outside the submitted work and participation in advisory boards from GSK and PurelMS, outside the submitted work. 


\section{REFERENCES}

1. Cutting GR. Cystic fibrosis genetics: from molecular understanding to clinical application. Nat Rev Genet. 2014;(November).

2. https://cftr2.org/.

3. Ramsey BW, Davies J, McElvaney NG, et al. A CFTR potentiator in patients with cystic fibrosis and the G551D mutation. N Engl J Med. 2011;365(18):1663-1672.

4. De Boeck K, Munck A, Walker S, et al. Efficacy and safety of ivacaftor in patients with cystic fibrosis and a non-G551D gating mutation. J Cyst Fibros. 2014;13(6):674-680.

5. Moss RB, Flume PA, Elborn JS, et al. Efficacy and safety of ivacaftor in patients with cystic fibrosis who have an Arg117His-CFTR mutation: a double-blind, randomised controlled trial. Lancet Respir Med. 2015;3(7):524-533.

6. Wainwright CE, Elborn JS, Ramsey BW, et al. Lumacaftor-Ivacaftor in patients with cystic fibrosis homozygous for Phe508del CFTR. N Engl J Med. May 2015.

7. Rowe SM, Daines C, Ringshausen FC, et al. Tezacaftor-Ivacaftor in residual-function heterozygotes with cystic fibrosis. N Engl J Med. November 2017:NEJMoa1709847.

8. Taylor-Cousar JL, Munck A, McKone EF, et al. Tezacaftor-Ivacaftor in patients with cystic fibrosis homozygous for Phe508del. N Engl J Med. November 2017:NEJMoa1709846.

9. Keating D, Marigowda G, Burr L, et al. VX-445-Tezacaftor-Ivacaftor in patients with cystic fibrosis and one or two Phe508del alleles. N Engl J Med. 2018;379(17):1612-1620.

10. Sato T, Clevers H. Growing self-organizing mini-guts from a single intestinal stem cell: mechanism and applications. Science (80- ). 2013;340(6137):1190-1194.

11. Dekkers JF, Wiegerinck $\mathrm{CL}$, de Jonge $\mathrm{HR}$, et al. A functional CFTR assay using primary cystic fibrosis intestinal organoids. Nat Med. 2013;19(7):939-945.

12. Dekkers JF, Berkers G, Kruisselbrink E, et al. Characterizing responses to CFTR-modulating drugs using rectal organoids derived from subjects with cystic fibrosis. Sci Transl Med. 2016;8(344):344ra84.

13. Berkers G, van Mourik P, Vonk AM, et al. Rectal organoids enable personalized treatment of cystic fibrosis. Cell Rep. 2019;26(7):1701-1708.e3.

14. de Winter-de Groot KM, Janssens HM, van Uum RT, et al. Stratifying infants with cystic fibrosis for disease severity using intestinal organoid swelling as a biomarker of CFTR function. Eur Respir J. 2018;52(3):1702529.

15. Dekkers JF, Van Mourik P, Vonk AM, et al. Potentiator synergy in rectal organoids carrying S1251N, G551D, or F508del CFTR mutations. J Cyst Fibros. May 2016.

16. Melin P, Thoreau V, Norez C, Bilan F, Kitzis A, Becq F. The cystic fibrosis mutation G1349D within the signature motif LSHGH of NBD2 abolishes the activation of CFTR chloride channels by genistein. Biochem Pharmacol. 2004;67(12):2187-2196.

17. Wang W, Bernard K, Li G, Kirk KL. Curcumin opens cystic fibrosis transmembrane conductance regulator channels by a novel mechanism that requires neither ATP binding nor dimerization of the nucleotide-binding domains. J Biol Chem. 2007;282(7):4533-4544.

18. Eckford PDW, Li C, Ramjeesingh M, Bear CE. Cystic Fibrosis Transmembrane Conductance Regulator (CFTR) Potentiator VX-770 (Ivacaftor) Opens the Defective Channel Gate of Mutant CFTR in a Phosphorylation-dependent but ATP-independent Manner. J Biol Chem. 2012;287(44):36639-36649.

19. Sohma Y, Yu Y-C, Hwang T-C. Curcumin and genistein: the combined effects on disease-associated CFTR mutants and their clinical implications. Curr Pharm Des. 2013;19(19):3521-3528.

20. Yu Y-C, Miki H, Nakamura Y, et al. Curcumin and genistein additively potentiate G551DCFTR. J Cyst Fibros. 2011;10(4):243-252. 
21. Dey I, Shah K, Bradbury NA. Natural compounds as therapeutic agents in the treatment cystic fibrosis. J Genet Syndr gene Ther. 2016;7(1).

22. Van Goor F, Hadida S, Grootenhuis PDJ, et al. Rescue of CF airway epithelial cell function in vitro by a CFTR potentiator, VX-770. Proc Natl Acad Sci. 2009;106(44):18825-18830.

23. Dutch Cystic Fibrosis Foundation. Nederlandse cystic fibrosis registratie 2018. 2019.

24. Standardization of spirometry, 1994 update. American Thoracic Society. Am J Respir Crit Care Med. 1995;152(3):1107-1136.

25. Koopman M, Zanen P, Kruitwagen CLJJ, van der Ent CK, Arets HGM. Reference values for paediatric pulmonary function testing: The Utrecht dataset. Respir Med. 2011;105(1):15-23.

26. Quanjer PH, Stanojevic S, Cole TJ, et al. Multi-ethnic reference values for spirometry for the 3-95-yr age range: the global lung function 2012 equations. Eur Respir J. 2012;40(6):13241343.

27. Dekkers R, Vijftigschild LAW, Vonk AM, et al. A bioassay using intestinal organoids to measure CFTR modulators in human plasma. J Cyst Fibros. 2015;14(2):178-181.

28. Summary of product characteristics - Kalydeco. 2018:1-76. https:/ / www.ema.europa.eu/ documents/product-information/kalydeco-epar-product-information_en.pdf.

29. Graeber SY, Dopfer C, Naehrlich L, et al. Effects of Lumacaftor/Ivacaftor therapy on CFTR function in Phe508del homozygous patients with cystic fibrosis. Am J Respir Crit Care Med. January 2018:rccm.201710-19830C.

30. Sasaki H, Sunagawa $Y$, Takahashi $\mathrm{K}$, et al. Innovative preparation of curcumin for improved oral bioavailability. Biol Pharm Bull. 2011;34(5):660-665.

31. Kanai M, Imaizumi A, Otsuka $Y$, et al. Dose-escalation and pharmacokinetic study of nanoparticle curcumin, a potential anticancer agent with improved bioavailability, in healthy human volunteers. Cancer Chemother Pharmacol. 2012;69(1):65-70.

32. Vareed SK, Kakarala M, Ruffin MT, et al. Pharmacokinetics of curcumin conjugate metabolites in healthy human subjects. Cancer Epidemiol Biomarkers Prev. 2008;17(6):1411-1417.

33. Cheng AL, Hsu CH, Lin JK, et al. Phase I clinical trial of curcumin, a chemopreventive agent, in patients with high-risk or pre-malignant lesions. Anticancer Res. 2001;21(4B):28952900.

34. Dhillon N, Aggarwal BB, Newman RA, et al. Phase Il trial of curcumin in patients with advanced pancreatic cancer. Clin Cancer Res. 2008;14(14):4491-4499.

35. Shoba G, Joy D, Joseph T, Majeed M, Rajendran R, Srinivas P. Influence of piperine on the pharmacokinetics of curcumin in animals and human volunteers. Planta Med. 1998;64(04):353-356.

36. Ullmann U, Metzner J, Frank T, Cohn W, Riegger C. Safety, tolerability, and pharmacokinetics of single ascending doses of synthetic genistein (Bonistein) in healthy volunteers. Adv Ther. 2005;22(1):65-78.

37. Ullmann U, Oberwittle H, Grossmann M, Riegger C. Repeated oral once daily intake of increasing doses of the novel synthetic genistein product Bonistein in healthy volunteers. Planta Med. 2005;71(10):891-896.

38. Metzner JE, Frank T, Kunz I, Burger D, Riegger C. Study on the pharmacokinetics of synthetic genistein after multiple oral intake in post-menopausal women. Arzneimittelforschung. 2009;59(10):513-520.

39. Evans M, Elliott JG, Sharma P, Berman R, Guthrie N. The effect of synthetic genistein on menopause symptom management in healthy postmenopausal women: a multi-center, randomized, placebo-controlled study. Maturitas. 2011;68(2):189-196. 


\section{SUPPLEMENTALS}

Supplemental Figure S1. Trial design study 1
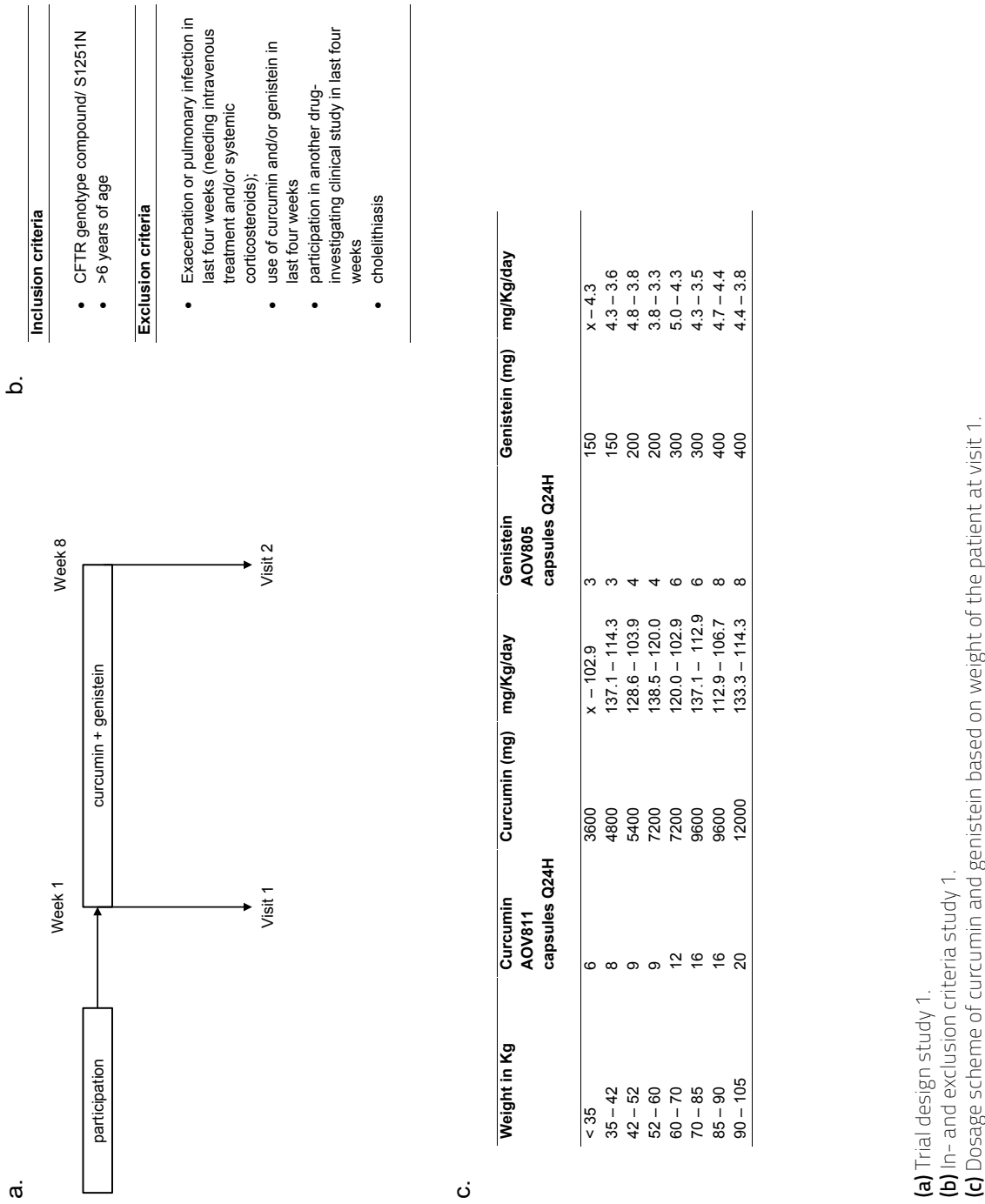
Supplemental Figure S2. Trial design study 2

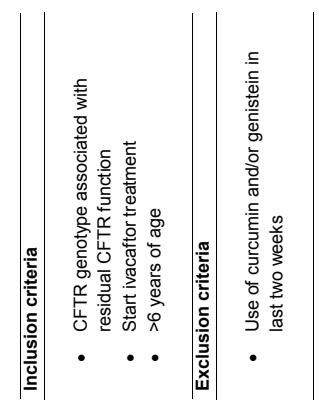

ف
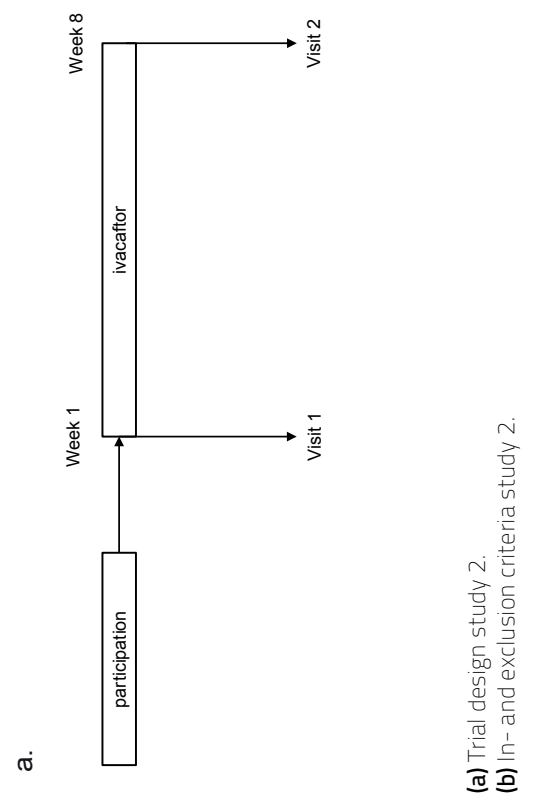
Supplemental Figure S3. Trial design study 3
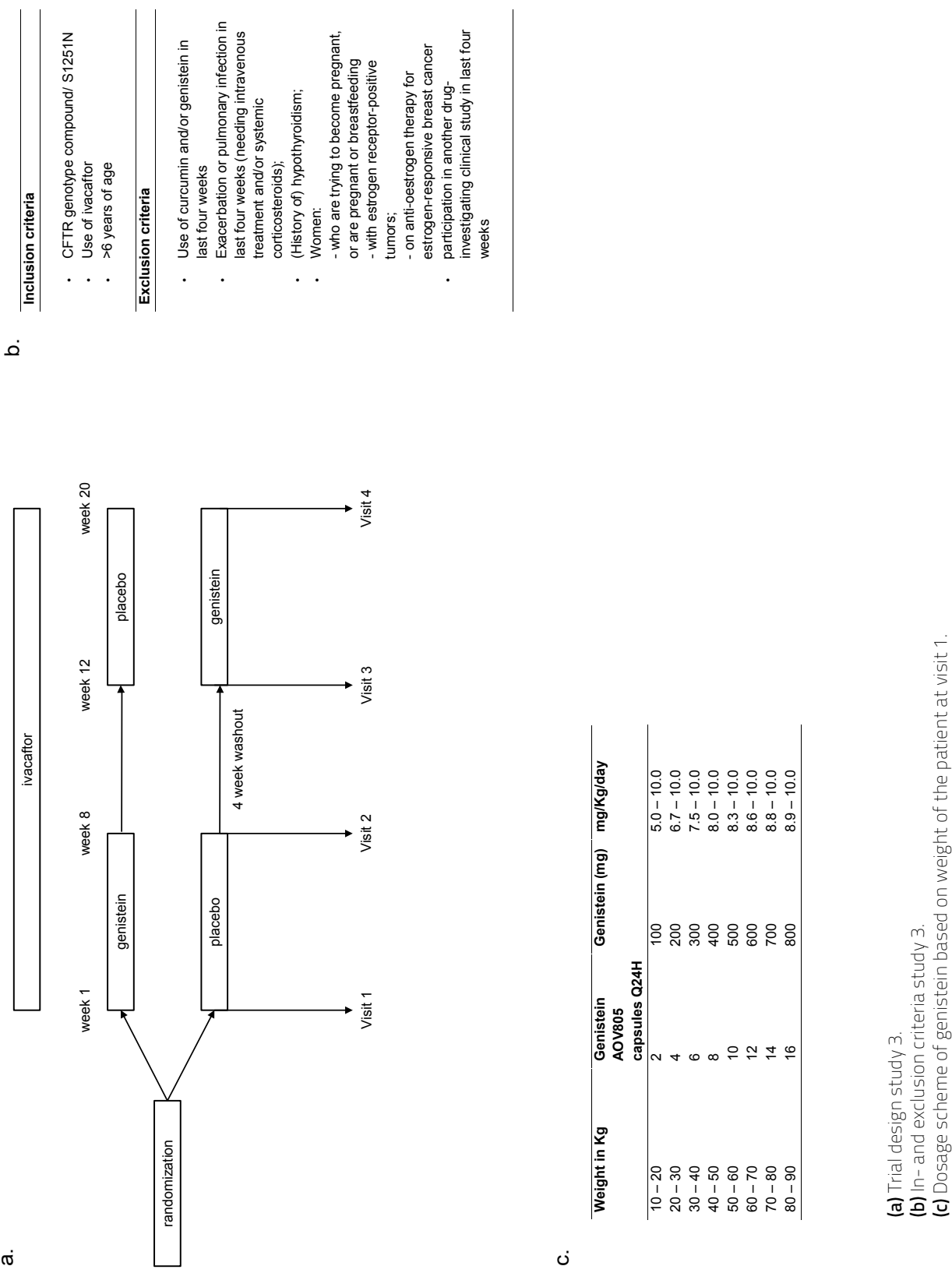

$\dot{0}$ 
Supplemental Figure S4. Consort diagrams for all three studies

68
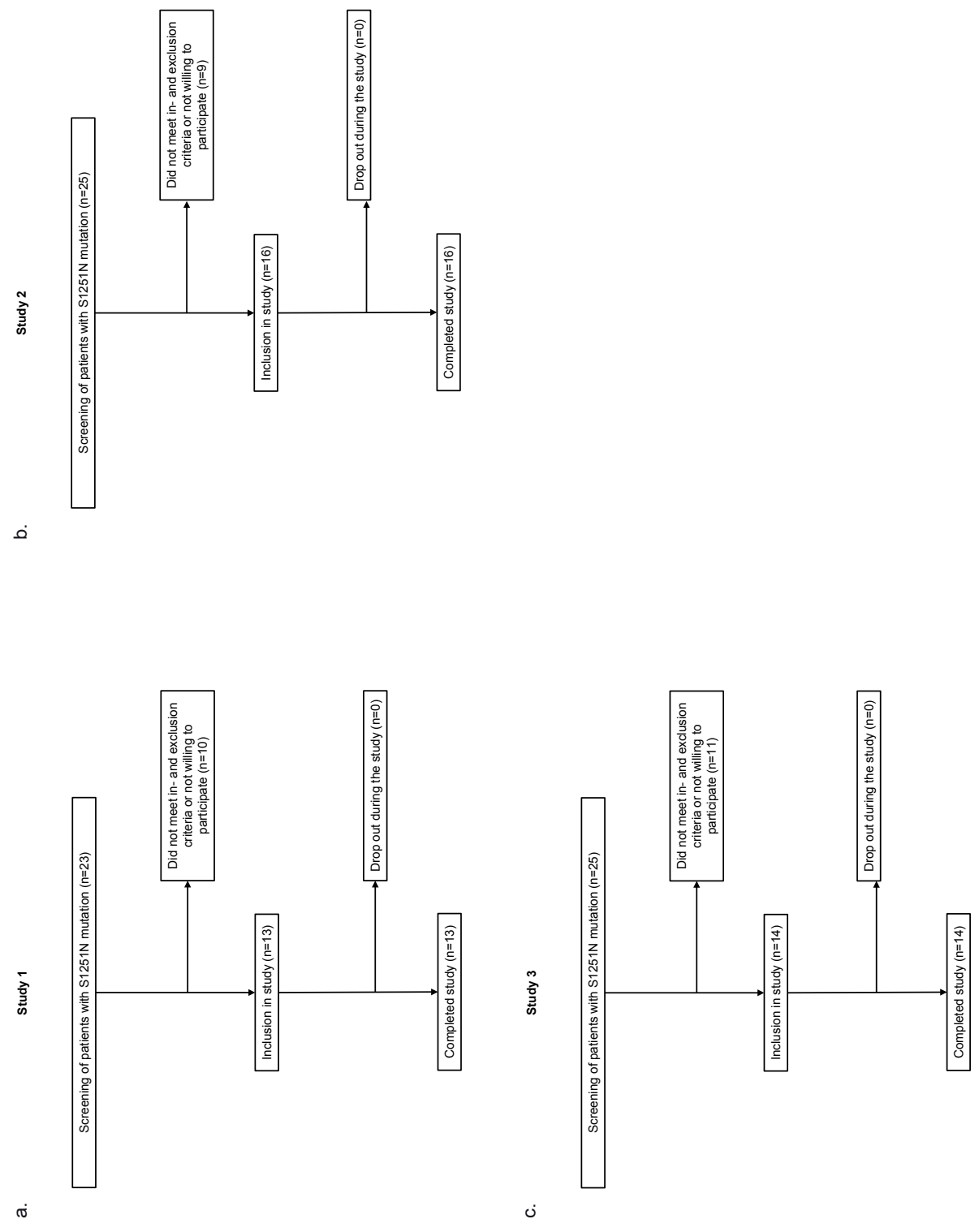
Supplemental Figure S5. Spaghetti plot of change in ppFEV1 in three studies

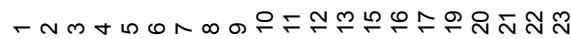

$\phi \phi+\phi \phi \phi \phi \phi \phi \phi t+* \phi \phi \phi \phi \phi+t$
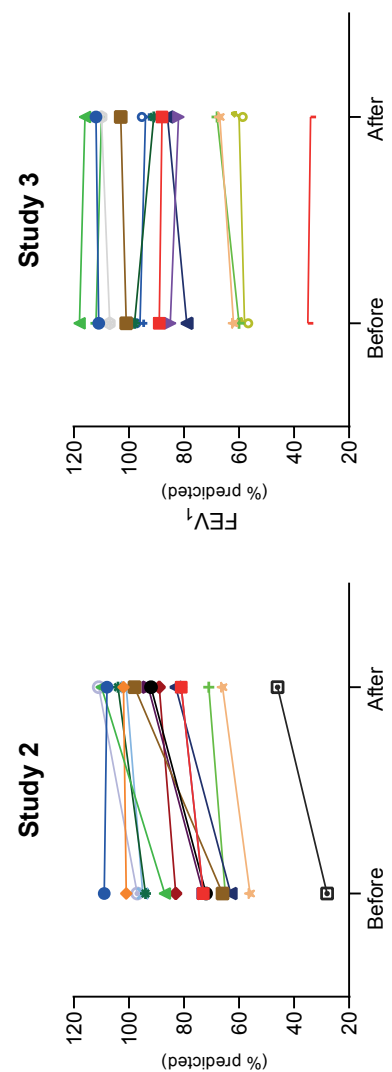

${ }^{\llcorner} \wedge \exists \exists$

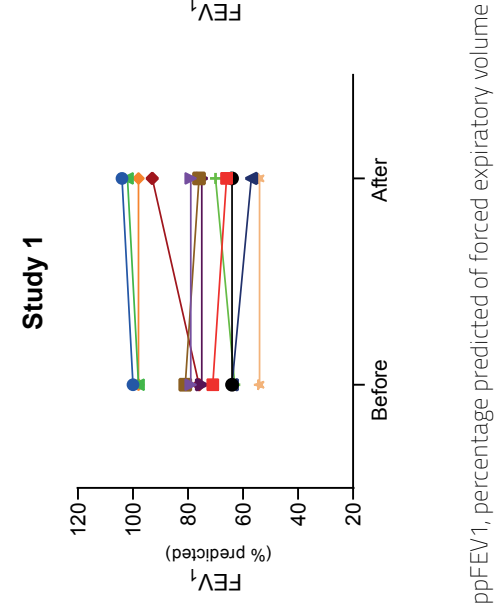


Supplemental Table S1. Product specifications curcumin and genistein

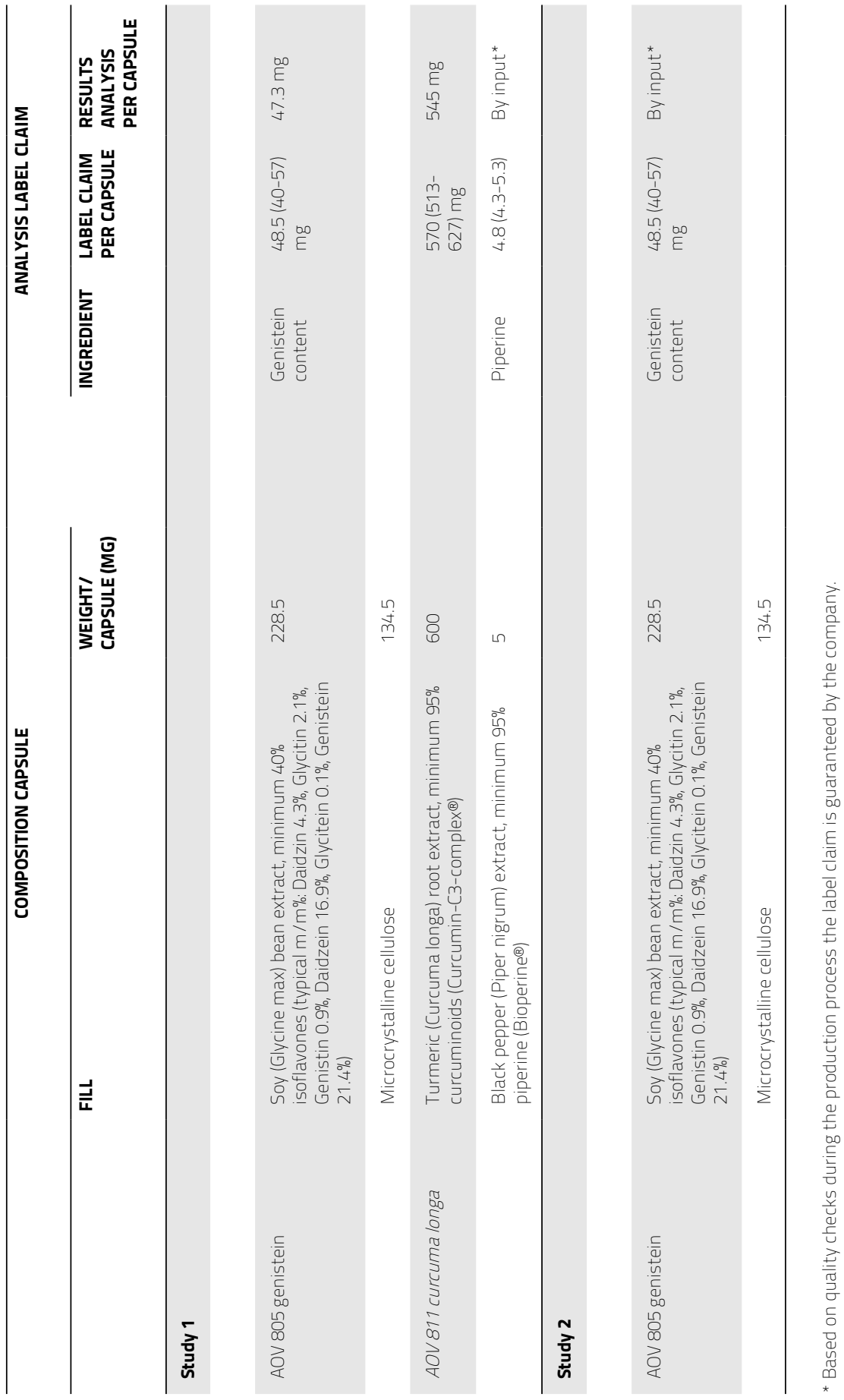




\section{Supplemental Table S2. Methods measuring plasma concentration}

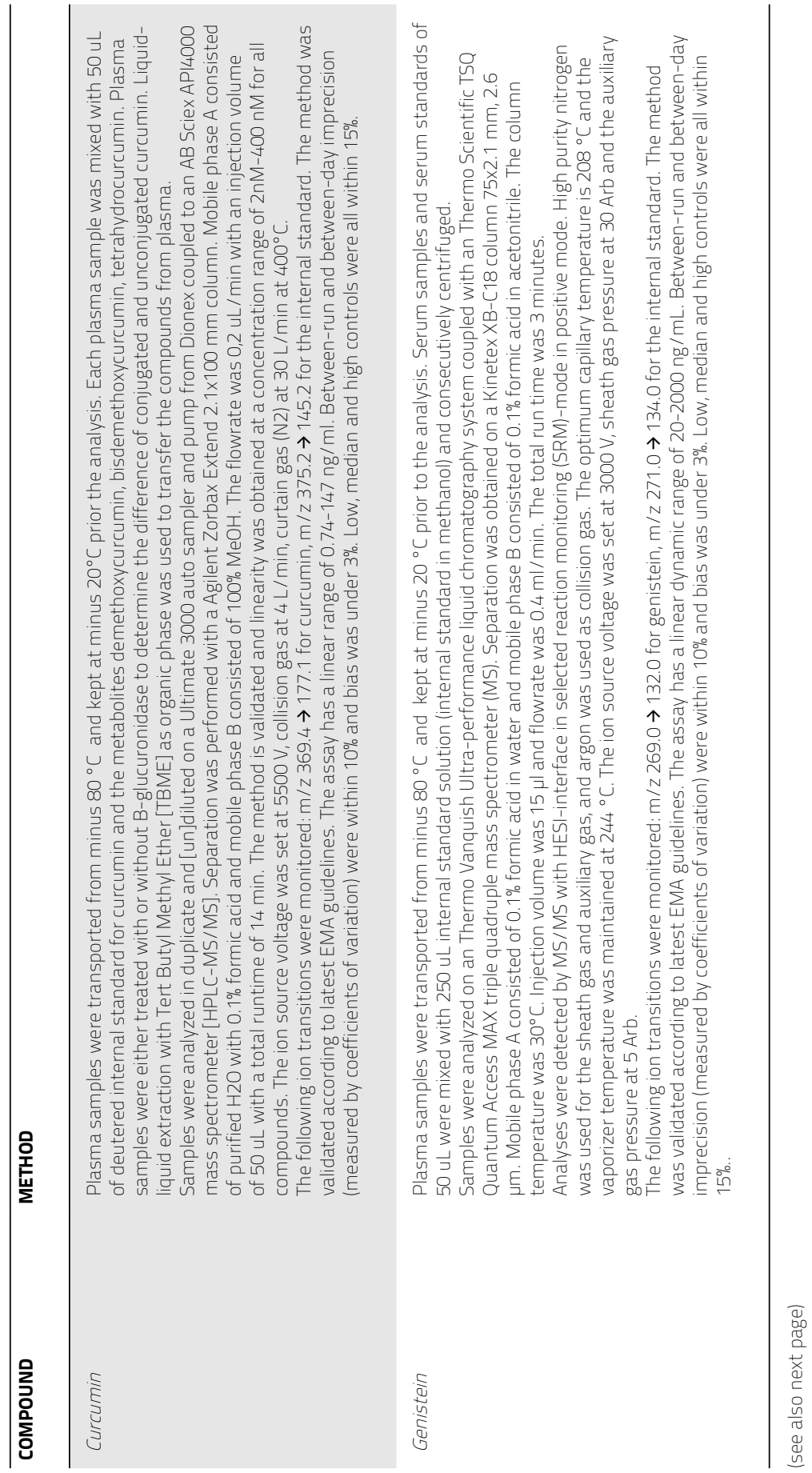


Chapter 3

Supplemental Table S2. Methods measuring plasma concentration

72

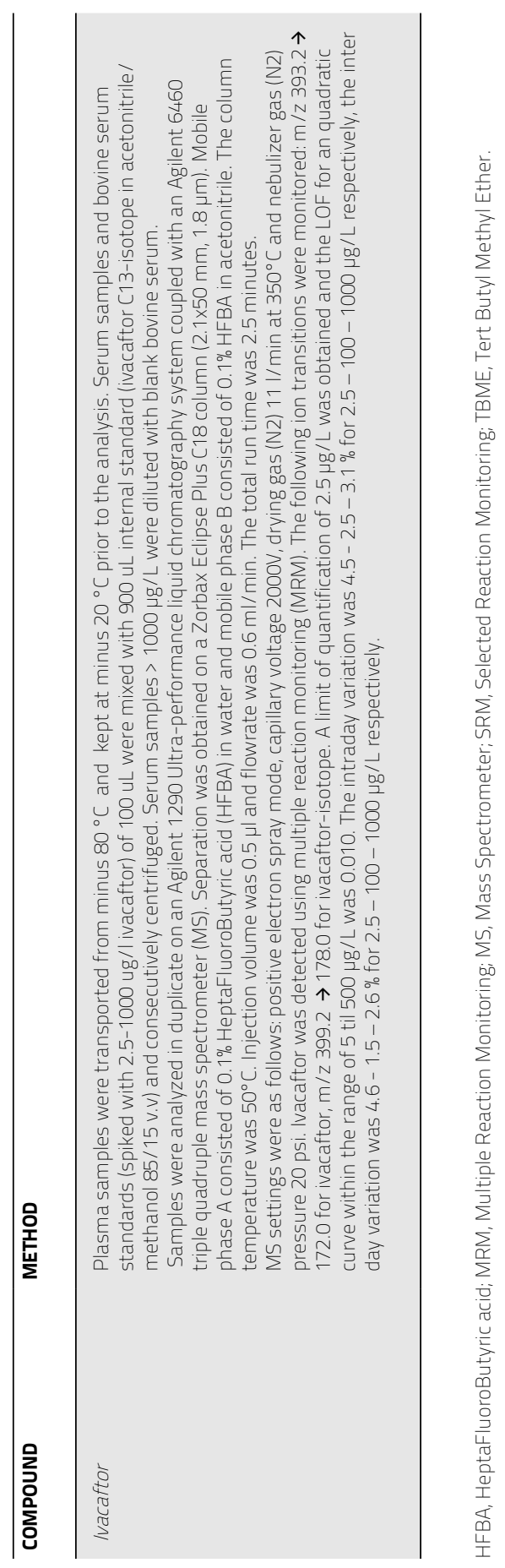


Supplemental Table S3. Plasma concentration of curcumin, genistein and their metabolites in study 1; comparison with literature data

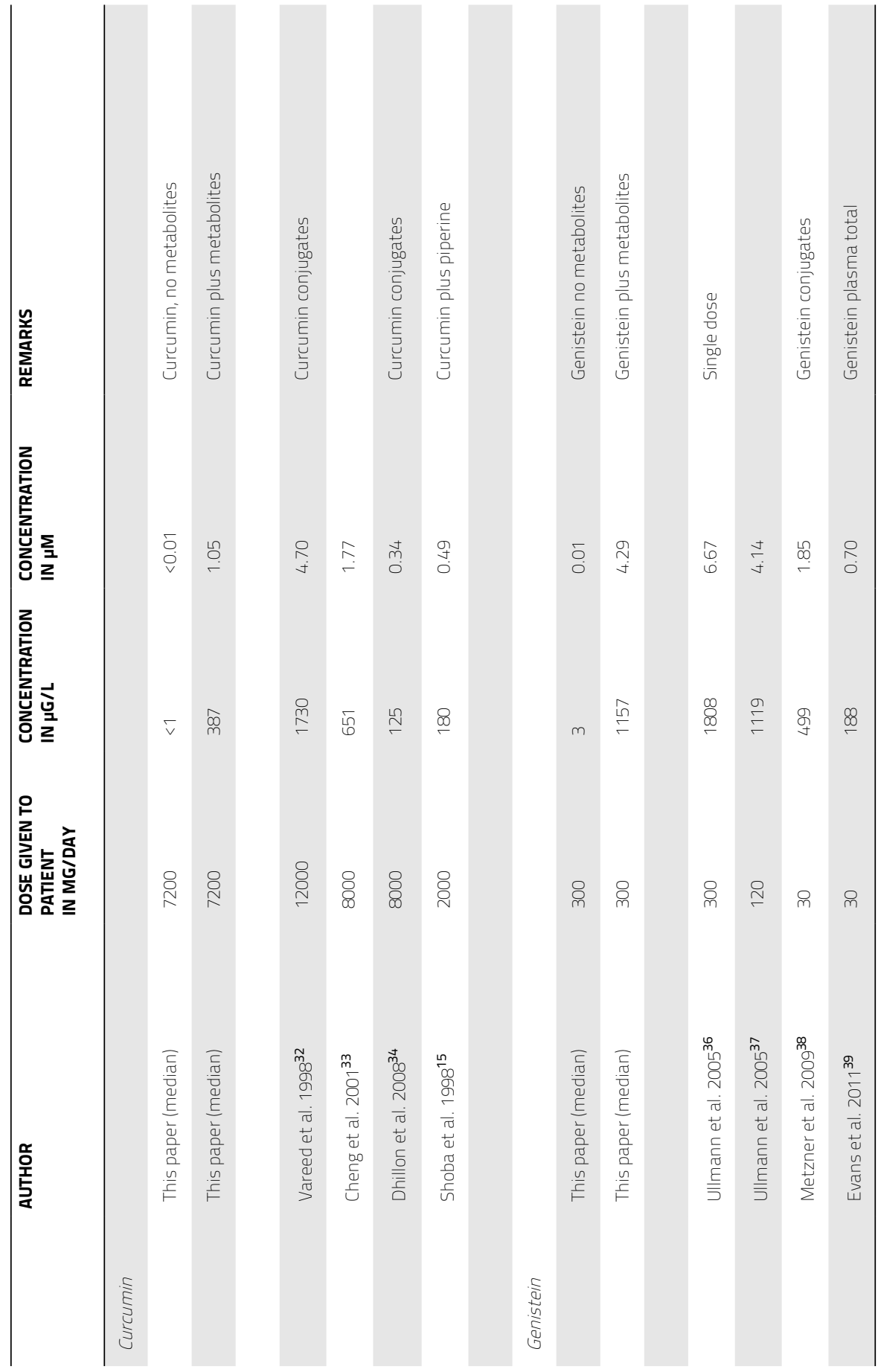




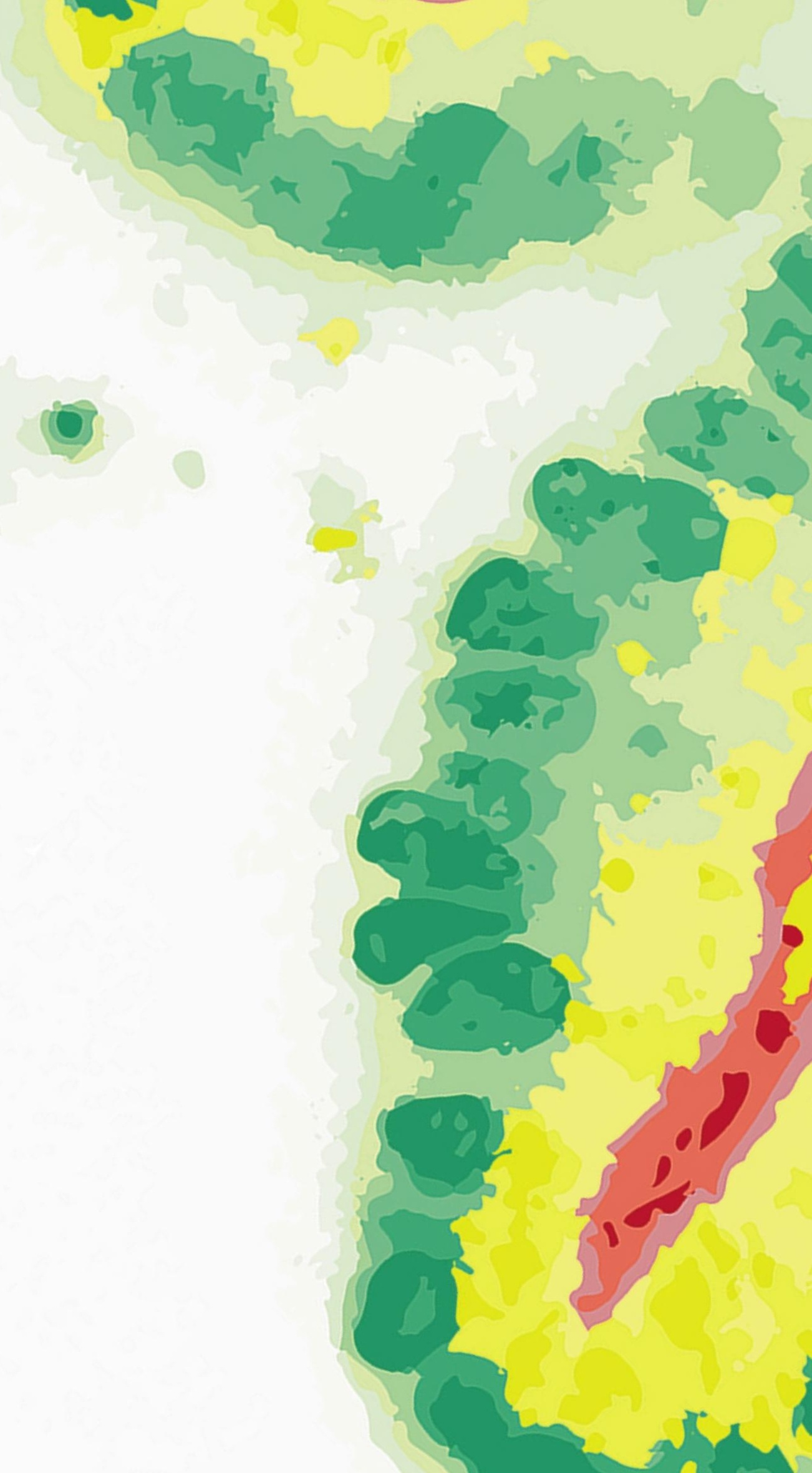




\section{CHAPTER 4}

\section{IVACAFTOR RESTORES FGF19 REGULATED BILE ACID HOMEOSTASIS IN CYSTIC FIBROSIS PATIENTS WITH AN S1251N OR A G551D GATING MUTATION}

Ivo P. van de Peppel*, Marcela Doktorova*, Gitte Berkers, Hugo R. de Jonge, Roderick H.J.

Houwen, Henkjan J. Verkade, Johan W. Jonker, Frank A.J.A. Bodewes

* These authors contributed equally 


\section{ABSTRACT}

Objective: Disruption of the enterohepatic circulation of bile acids (BAs) is part of the gastrointestinal phenotype of cystic fibrosis (CF). Ivacaftor (VX-770), a cystic fibrosis transmembrane conductance regulator (CFTR) potentiator, improves pulmonary function in CF patients with class III gating mutations. We studied the effect of ivacaftor on the enterohepatic circulation by assessing markers of BA homeostasis and their changes in CF patients.

Methods: In CF patients with an S1251N mutation ( $\mathrm{N}=16$; age 9-35 years S125N study/ NTR4873) or a G551D mutation ( $N=101$; age 10-24 years; GOAL study/ NCT01521338) we analyzed plasma fibroblast growth factor 19 (FGF19) and $7 \alpha$-hydroxy-4-cholesten3-one (C4) levels, surrogate markers for intestinal BA absorption and hepatic synthesis, respectively, before and after treatment with ivacaftor.

Results: At baseline, median FGF19 was lower $(52 \%$ and 53\%, P . .001) and median C4 higher $(350 \%$ and $364 \%, P<.001)$, respectively, for the S1251N and G551D mutation patient groups compared to healthy controls. Treatment with ivacaftor significantly increased FGF19 and reduced C4 levels towards normalization in both cohorts but this did not correlate with CFTR function in other organs, as measured by sweat chloride levels or pulmonary function.

Conclusion: We demonstrate that patients with CFTR gating mutations display interruption of the enterohepatic circulation of BAs reflected by lower FGF19 and elevated C4 levels. Treatment with ivacaftor partially restored this disruption of BA homeostasis. The improvement did not correlate with established outcome measures of $\mathrm{CF}$, suggesting involvement of modulating factors of CFTR correction in different organs. 


\section{INTRODUCTION}

Cystic fibrosis (CF) is the most common autosomal recessive disorder in the Caucasian population and is characterized by the production of abnormally thick, viscous mucus in the lungs, digestive system and various other tissues., ${ }^{1,2}$ The defect is caused by mutations in the cystic fibrosis transmembrane conductance regulator (CFTR/ABCC7) gene that encodes a chloride channel protein. ${ }^{3}$ The diagnosis of CF is confirmed by an increased sweat chloride concentration which is considered a surrogate for the CFTR protein chloride channel function and/or by genetic testing. CF patients primarily suffer from progressive pulmonary disease as determined by a reduced forced expiratory volume in $1 \mathrm{~s}\left(\mathrm{FEV}_{1}\right)$. Additionally, they frequently display exocrine pancreatic insufficiency, poor growth and weight gain resulting in a low body mass index (BMI) and intestinal complications like e.g. distal intestinal obstruction syndrome. ${ }^{1}$

Gastrointestinal problems are common among CF patients. Increased fecal bile acid (BA) loss is an intrinsic part of the gastrointestinal phenotype of $C F$, found consistently in both CF patients and CF animal models.,5 Compared to healthy controls, CF patients have an up to three-fold increase in fecal BA loss. This fecal BA loss is independent of CF-related exocrine pancreatic insufficiency and intestinal fat malabsorption. ${ }^{4,6}$ The higher fecal BA excretion indicates a partial interruption of the enterohepatic circulation of BA and is associated with a compensatory increase of de novo hepatic BA synthesis. ${ }^{6}$ Adequate BA homeostasis is essential to hepatic, intestinal and metabolic function. ${ }^{7}$ CF patients regularly display complications such as colonic dysbiosis, small intestinal bacterial overgrowth (SIBO). ${ }^{8}$ The disruption in BA homeostasis, may contribute to these complications, and drugs affecting BA homeostasis could potentially benefit CF patients. ${ }^{9}$

The enterohepatic circulation comprises hepatobiliary secretion and intestinal reabsorption of BA and is controlled via a negative feedback system. ${ }^{10}$ After uptake in the distal small intestine, $B A$ s bind to the Farnesoid $X$ receptor (FXR) which is localized in the enterocytes and induces the expression and secretion of fibroblast growth factor 19 (FGF19) into the portal circulation. ${ }^{11}$ In the liver, FGF19 binds to the FGF receptor 4(FGFR4), leading to suppression of the cholesterol 7 $\alpha$-hydroxylase (CYP7A1) gene, encoding the rate-limiting enzyme in BA synthesis. The protein activity of CYP7A1 can be inferred from the concentration of the BA intermediate, $7 \alpha$-hydroxy-4-cholesten-3-one (C4), that is used as a surrogate marker for BA synthesis. ${ }^{12}$ Clinically, altered plasma levels of FGF19 and C4 have been used as markers for disrupted BA metabolism. For example patients with primary bile acid diarrhea (BAD) display BA malabsorption and increased BA synthesis, resulting in decreased plasma FGF19 levels, and elevated plasma C4 levels. ${ }^{13-16}$ Based on the CF phenotype of BA malabsorption, it is expected that FGF19 levels are low and C4 levels are high in CF patients, however, this has never been documented. 
Ivacaftor, a drug developed for the treatment of CF patients, potentiates CFTR channel activity and thereby enhances chloride transport in patients with class III gating mutations, such as the S1251N and the G551D CFTR missense mutations. ${ }^{17,18}$ In The Netherlands, the $51251 \mathrm{~N}$ mutation is the most common class III mutation with an occurrence of $1.2 \%$ in $2016 .{ }^{19}$ The G551D mutation is present in $\sim 4 \%$ of all CF patients in the United States and is the third most common CFTR mutation worldwide. ${ }^{20}$ In randomized placebo-controlled trials, it has been shown that ivacaftor improves sweat chloride levels, pulmonary function (FEV1) and BMI in G551D and S1251N patients. ${ }^{21-23}$ Recently, a study also showed the efficacy of ivacaftor in the intestine where it improved the small intestinal $\mathrm{pH}$ after gastric emptying by increasing bicarbonate secretion. ${ }^{24}$

The NTR4873 clinical trial was an open label observational study that assessed the effects of ivacaftor in a small Dutch cohort of patients with the S1251N mutation. In this study, fecal and plasma samples were obtained before and eight weeks after treatment with ivacaftor.

The multicenter clinical G551D observational (GOAL) study was a six months followup, phase 4 study exploring the therapeutic effect of ivacaftor in patients with a G551D mutation. The GOAL study confirmed, in these specific CF patients, the positive treatment effects observed earlier in the original clinical trials for ivacaftor on sweat chloride, FEV1 and BMI. ${ }^{25}$ Within the GOAL study, blood samples were taken and stored before and at six months after the start of the treatment.

We aimed to evaluate the effects of CFTR modulation on the gastrointestinal tract. Specifically, we investigated whether ivacaftor treatment induces a recovery of the enterohepatic circulation of BAs in CF patients as reflected by the effects on FGF19 and C4 plasma levels.

\section{METHODS}

\section{Subjects}

For the S1251N mutation study, samples were obtained before and 2 months after treatment with ivacaftor. A total of 16 patients (age 9-35 years) were recruited. All patients had an $51251 \mathrm{~N}$ mutation at one allele. At the other allele 12 patients had a deltaF508 mutation, 2 patients a R117H mutation, 1 patient an 1717-IGA mutation and 1 an A455E mutation. $13(81 \%)$ patients were reported to be exocrine pancreatic insufficient. Plasma samples before and after ivacaftor treatment were obtained for 14 participants. From 10 patients, random fecal samples were also obtained before and at 2 months after starting treatment with ivacaftor. Clinical data including age, BMI, FEV1 scores, sweat chloride levels, comorbidity and medication use was gathered for all patients. The 
ethics committees of the University Medical Center Utrecht and 3 other Medical Centers (Erasmus MC, Rotterdam; AMC Amsterdam; HAGA The Hague) approved this study, and informed consent was obtained from all participating subjects.

Plasma samples from 101 CF patients (age 10-24 years) before and after six months of ivacaftor treatment were obtained from the G551D observational (GOAL) study (clinicaltrials.gov number, NCT01521338). ${ }^{25}$ The protocol was reviewed and approved by the GOAL study review board. Patients enrolled in this study were six years and older, had a G551D gating mutation on at least one allele and no prior history of ivacaftor use. Patients using ursodeoxycholic acid (UDCA) were excluded from the GOAL cohort. Available BMI, FEV1 scores, and sweat chloride levels before and after ivacaftor treatment were obtained from the GOAL study database. Additional ethical approval was obtained from the medical ethical committee of the University Medical Center Groningen. Two patients were excluded based on a baseline sweat chloride level of $\leq 39 \mathrm{mEq} / \mathrm{L}$, which indicated a sweat chloride level below the upper limit of normal. ${ }^{26}$ The final data analysis was performed on the data of the remaining 99 patients.

\section{Control group}

The control group consisted of non-fasted plasma samples of 120 healthy subjects, aged 18-50 years and with a BMI between 18.5 and 30, from the Dutch multidimensional cohort study and biobank LifeLines. ${ }^{27}$

\section{Plasma levels of FGF19 and C4}

Plasma samples were stored at $-20{ }^{\circ} \mathrm{C}$. FGF19 levels were determined using a quantitative sandwich enzyme-linked immunosorbent assay (Human FGF-19 Quantikine enzyme-linked immunosorbent assay (ELISA) kit, cat no. DF1900; R\&D systems). Plasma C4 levels were determined using high-performance liquid chromatographytandem mass spectrometry (XLC-MS/MS). ${ }^{28}$ All the samples were assayed in duplicate.

\section{Fecal and plasma bile acids}

Fecal samples were weighed, freeze-dried and mechanically homogenized. Samples were then solubilized in $1 \mathrm{ml}$ of methanol/0.1 $\mathrm{N}$ sodium hydroxide $(3: 1)$ at $80{ }^{\circ} \mathrm{C}$ for 2 h. As an internal standard $15 \mathrm{nmol}$ of $5 \beta$-Cholanic acid $7 \alpha, 12 \alpha$ diol was added. Next, bile acids were extracted using Sep Pak C-18 columns (Mallinckrodt Baker, Deventer, The Netherlands) and eluted with 75\% methanol followed evaporation at 65C under a stream of nitrogen. Dried samples were methylated with methanol/acetyl chloride for 25 min at $55^{\circ} \mathrm{C}$. Samples were dried under a stream of nitrogen and trimethylsilylated with a mixture of pyridine, $\mathrm{N}, \mathrm{O}$-Bis (trimethylsilyl) trifluoroacetamide and trimethylchlorosilane. The bile acid samples were then diluted in $150 \mu$ of heptane and analyzed by GC (Agilent 
6890, Amstelveen, the Netherlands) using a CPSil 19 capilliary column (25mx0.25mmx0.2 $\mu \mathrm{m})$ (Chrompack, Middelburg, The Netherlands) The total amount of bile acids was calculated as the sum of the individually quantified bile acids. Plasma bile acid profiles were measured using liquid chromatography-mass spectrometry (LC-MS). For the analysis $25 \mu$ of plasma was used to which a $250 \mu$ internal standard solution was added. Samples were centrifuged at $15800 \times \mathrm{g}$ and the supernatant poured into a clean glass tube. The fluid was evaporated under nitrogen at $40 \mathrm{C}$. Before measuring samples were reconstituted in $200 \mu \mathrm{l} 50 \%$ methanol in water, vortexed and centrifuged for $3 \mathrm{~min}$ at $1800 \times \mathrm{g}$. The supernatant was transferred into a $0.2 \mu \mathrm{m}$ spin-filter and centrifuged at $2000 \times \mathrm{g}$ for $10 \mathrm{~min}$. After filtering, the samples were transferred into LC-MS vials and analyzed (10 $\mu$ injection volume). For the quantitative determination of bile acids we used a Nexera X2 Ultra High Performance Liquid Chromatography system (SHIMADZU, Kyoto, Japan), coupled to a SCIEX QTRAP 4500 MD triple quadrupole mass spectrometer (SCIEX, Framingham, MA, USA) (UHPLC-MS/MS). The LC-MS/MS system is controlled by Analyst MD 1.6.2 software. Bile acids were separated with a ACQUITY UPLC BEH C18 Column $(1,7 \mu \mathrm{m} \times 2,1 \times 100 \mathrm{~mm})$ equipped with a ACQUITY UPLC BEH C18 VanGuard Pre-Column (1,7 $\mu \mathrm{m} \times 2,1 \times 5 \mathrm{~mm})$, (Waters, Milford, MA, USA). Separation was achieved in 28 min using $10 \mathrm{mM}$ ammonium acetate in $20 \%$ acetonitrile (mobile phase A) and 10 $\mathrm{mM}$ ammonium acetate in $80 \%$ acetonitrile (mobile phase $\mathrm{B}$ ), total flow rate: $0.4 \mathrm{ml} / \mathrm{min}$.

\section{Statistical analysis}

Statistical analysis was performed using SPSS Statistics 22 (International Business Machines, Armonk, New York). FGF19, C4 and fecal BA data were not normally distributed and are displayed as Tukey plots where boxes represent the median with interquartile range (IQR) and whiskers extend to the largest value or 1.5 times the IQR if the largest value extends that. A Mann-Whitney $U$ test was performed to compare CF patients versus controls. For the statistical analysis of treatment effect a Wilcoxon Signed Rank test was performed. Correlation analysis was performed only on the GOAL data as the sample size of the $51251 \mathrm{~N}$ study was considered too small for a reliable correlation analysis. For the relationship between FGF19 and C4 measurements a Spearman's rank coefficient was used as data were not normally distributed. However, all changes in parameters were normally distributed (determined by Q-Q plot) and therefore these correlations were analyzed using a Pearson R correlation coefficient. $\mathrm{P}<.05$ was considered significant. All authors had access to the study data and have reviewed and approved the final manuscript. 


\section{RESULTS}

\section{Ivacaftor improves sweat chloride level, BMI and FEV1 scores in CF patients with an S1251N mutation}

Table 1 shows the patient characteristics of the control subjects and CF patients with an $5125 \mathrm{~N}$ mutation before and after 2 months of ivacaftor treatment. Of the $14 \mathrm{CF}$ patients analyzed for FGF19 and C4 data, 10 were male. In the control group 60 of the 120 subjects were male. In line with improved CFTR function, the median sweat chloride level significantly decreased after ivacaftor treatment. Median FEV1 and BMI significantly increased, showing clinical efficacy of ivacaftor treatment.

The median age of the controls was 33 years which was significantly higher than in the CF group (16 years) at baseline ( $P<.001)$. Controls had a median BMI of $24.4 \mathrm{~kg} / \mathrm{m} 2$ which was significantly higher than in CF patients at baseline $(19.0 \mathrm{~kg} / \mathrm{m} 2, \mathrm{P}<.001)$ and after ivacaftor treatment $(20.1 \mathrm{~kg} / \mathrm{m} 2, \mathrm{P}<.001)$.

\section{Ivacaftor improves bile acid homeostasis in CF patients with an S1251N mutation}

Fig. 1 shows the changes in plasma FGF19 and C4 levels and fecal BA concentrations at 2 months after ivacaftor treatment. As a surrogate marker for BA absorption plasma FGF19 was measured. Fig. 1a shows the individual changes in FGF19 level of all patients with an S1251N mutation. One patient had diagnosed cystic fibrosis liver disease and received UDCA treatment. However, as the measured plasma parameters were similar to untreated patients, these results were included in the final analysis. No fecal samples were obtained for this patient. Median FGF19 level increased significantly from 45 to $113 \mathrm{pg} / \mathrm{ml}$ (increase in 12 of 14 patients, Fig. $1 \mathrm{~b}, \mathrm{P}=.003$ ). In accordance with the BA malabsorption phenotype of CF, median FGF19 levels in CF patients at baseline were significantly lower as compared to healthy controls ( 45 vs. 94 pg/ml, $\mathrm{P}<.001$ ). After 8 weeks of ivacaftor treatment median FGF19 levels were similar between CF patients and controls (113 vs. $94 \mathrm{pg} / \mathrm{ml}, \mathrm{P}=.91$ ). These results suggest that ivacaftor treatment induces an increased BA reabsorption in CF patients with an S1251N mutation.

FGF19 inhibits hepatic BA synthesis. We, therefore, determined plasma C4 levels as a surrogate marker for BA synthesis. Fig. 1c shows individual changes in plasma C4 levels. All except two patients showed a decrease in C4 level after 2 months of ivacaftor treatment. These two patients showed an increase of 6 and $4 \mathrm{ng} / \mathrm{ml}$. The latter subject was the same patient that showed the highest decrease in FGF19 level ( $-54 \mathrm{pg} / \mathrm{ml})$. The other subject showed despite the slight increase in C4 also an increase in FGF19 level (+83 pg/ml). In Fig. $1 d$ the median change in plasma C4 level is shown. Median plasma C4 levels decreased significantly from 49 to $24 \mathrm{ng} / \mathrm{ml}(\mathrm{P}=.001)$ suggesting a decrease in hepatic BA synthesis. While median C4 levels improved towards normality, values at 
Table 1. Basic characteristics of controls and CF patients before and after ivacaftor treatment in the S1251N study cohort

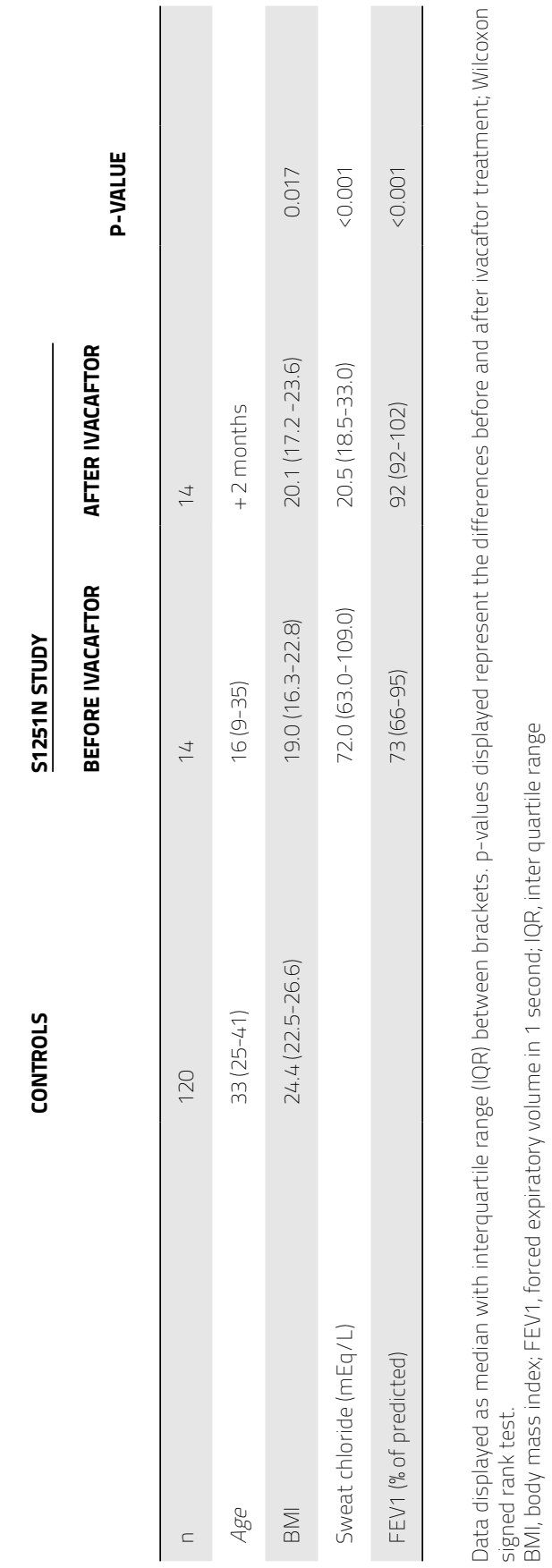


Figure 1. Improvement of BA metabolism markers after ivacaftor treatment in the S1251N study cohort
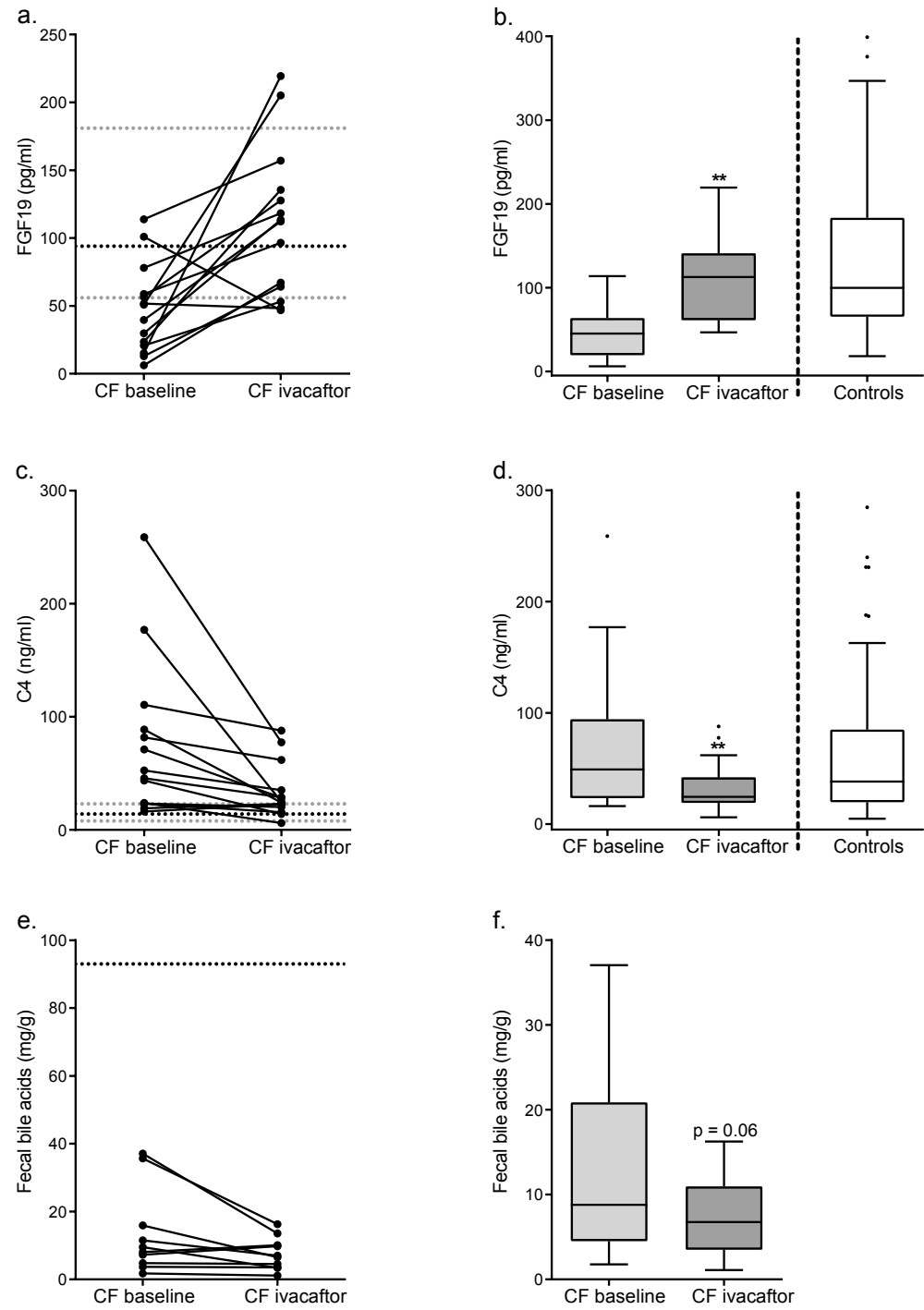

(a) change in plasma FGF19 of individual patients.

(b) change in plasma FGF19 on group level $n=14$.

(c) change in plasma $7 \alpha$-hydroxy-4-cholesten-3-one (C4) of individual patients.

(d) change in plasma C4 on group level $n=14$, dotted lines represent median in black and interquartile range (IQR) in dark grey of healthy controls.

(e) change in fecal bile acid (BA) concentrations of individual patients.

(f) change in fecal BA concentrations on group level $(n=10) .{ }^{* *} p<0,01$; Wilcoxon signed rank test. Data expressed as Tukey plots, outliers represented as separate dots. 
Table 2. Basic characteristics of controls and CF patients before and after ivacaftor treatment in the G551D study cohort

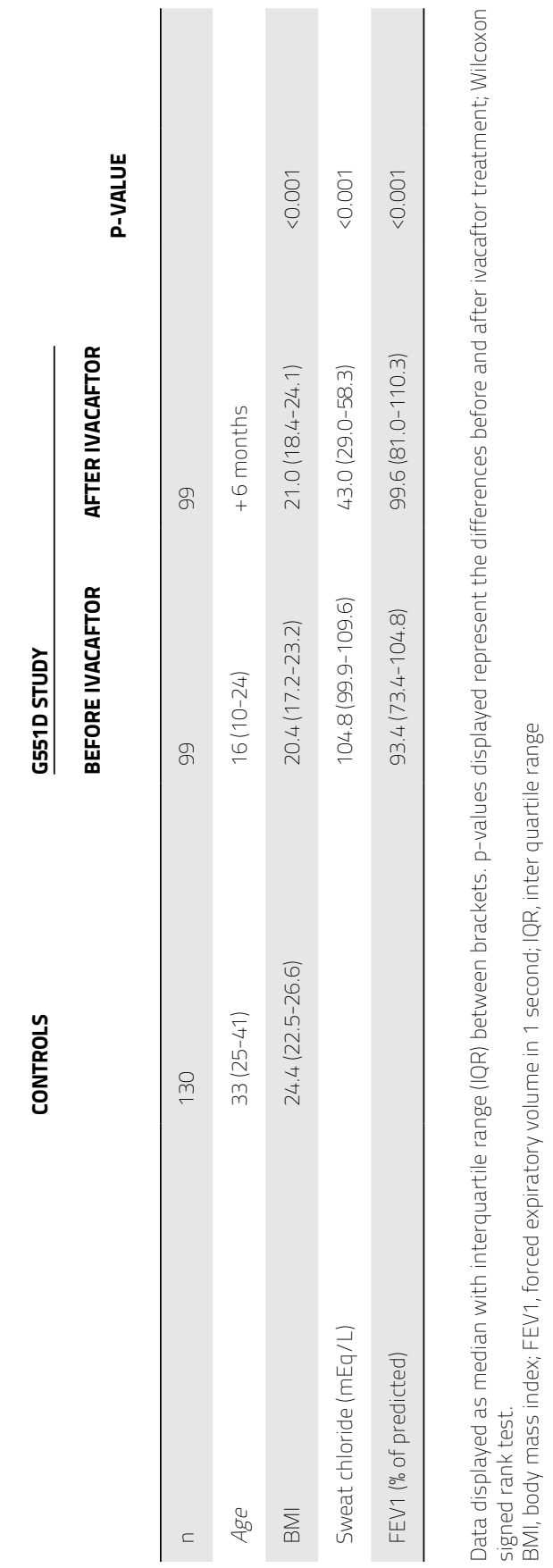


Figure 2. Effect of ivacaftor treatment on BA metabolism markers in the G551D study cohort
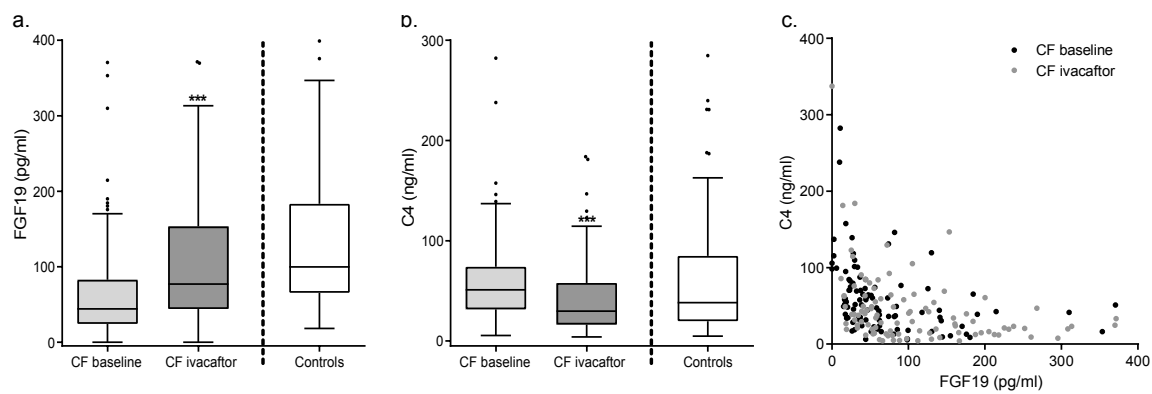

(a) change in FGF19 on group level and (b) change in C4 on group level ( $n=99)$. ${ }^{* * *} \mathrm{P}<$.001; Wilcoxon signed rank test. Data expressed as Tukey plots, outliers represented as separate dots, dotted lines represent median in black and interquartile range (IQR) in dark grey of healthy controls.

(c) C4 and FGF19 levels are similarly inversely correlated before (Spearman $r=-0.50, \mathrm{P}<.001$ ) and after (Spearman $r=-0.42, P<.001$ ) ivacaftor treatment, $n=99$.

baseline and after ivacaftor treatment were still significantly higher than control values (49 vs $14 \mathrm{ng} / \mathrm{ml} ; \mathrm{P}<.001$ and $24 \mathrm{ng} / \mathrm{ml} ; \mathrm{P}=.001$; respectively).

Fig. 1e shows individual changes in fecal BA concentrations. Random fecal samples before and after ivacaftor treatment were available for 10 patients. Fig. If shows the median change in fecal BA concentrations. Median fecal BA decreased after Ivacaftor treatment from 8.8 to $6.8 \mathrm{mg} / \mathrm{g}$ but the difference did not reach statistical significance $(P=.06)$. Fecal and plasma BA profiles were similar before and after treatment (supplemental Tables 1 and 2).

Together these results show that 2 months of ivacaftor treatment restores the enterohepatic circulation of BAs in CF patients with an S1251N mutation towards normality.

\section{Ivacaftor improves sweat chloride level, BMI and FEV1 scores in CF patients with a G551D mutation}

Table 2 shows the characteristics of the control patients and of the patients with a G551D mutation before and 6 months after treatment with ivacaftor. Improvements in sweat chloride level, $\mathrm{FEV}_{1}$ and BMI were published before by Rowe et al. ${ }^{25}$ Also in the G551D cohort, median age was significantly lower compared to the control group (16 vs. 33 years, $P<.001)$. BMI was also significantly lower before $(20.4 \mathrm{vs} .24 .4 \mathrm{~kg} / \mathrm{m} 2, \mathrm{P}<.001)$ and after ivacaftor treatment (21.0 vs. $24.4 \mathrm{~kg} / \mathrm{m} 2, \mathrm{P}<.001)$ compared to the control group. 
Of the 99 patients included, 49\% was male. More detailed baseline characteristics and the effect of ivacaftor on the improvement in BMI, FEV1 and sweat chloride level of this cohort have recently been published as part of the first GOAL study report. ${ }^{25}$

\section{Ivacaftor improves plasma FGF19 and C4 levels towards healthy control values in CF patients with a G551D mutation}

Fig. 2a shows the FGF19 levels of CF patients with a G551D mutation at baseline and after 6 months of ivacaftor treatment. At baseline median FGF19 levels were significantly lower in CF patients as compared to healthy controls (44vs.94 pg/ml; P . .001). After 6 months of ivacaftor treatment median FGF19 levels significantly increased from 44 to $77 \mathrm{pg} / \mathrm{ml}(\mathrm{P}<.001)$. An increase was observed in $73 \%$ of the ivacaftor treated patients. Ivacaftor treatment normalized FGF19 levels to healthy control values (77 vs. $94 \mathrm{pg} / \mathrm{ml}$, $P=.11)$, similar as found in patients with an $51251 \mathrm{~N}$ mutation.

Fig. $2 b$ shows the median levels of plasma C 4 before and after 6 months of ivacaftor treatment. At baseline median C4 levels, representing compensatory hepatic BA synthesis, were significantly higher in CF patients with a G551D mutation as compared to healthy controls (51 vs. $14 \mathrm{ng} / \mathrm{ml}, \mathrm{P}<.001$ ). In accordance with the observed increase in FGF19 level, C4 levels decreased in 70\% of patients and median C4 levels decreased from 51 to $30 \mathrm{ng} / \mathrm{ml}(\mathrm{P}<.001)$.

While median C4 levels improved towards normality after ivacaftor treatment, they still remained significantly higher than median levels of healthy control value (30 vs $14 \mathrm{ng} / \mathrm{ml}$, $P<.001)$. While the increase in FGF19 level was significantly correlated with a decrease in $C 4$ level $(r=-0.43, P<.0001)$, the decrease in C4 can only partially be explained by the increase in FGF19 ( $r 2=0.18$ ). Fig. $2 c$ shows the inverse correlation between $\mathrm{C} 4$ and FGF19 levels.

\section{Changes in FGF19 and C4 do not correlate with effects of ivacaftor on sweat chloride concentrations and FEV1}

An elevated sweat chloride level (Table 1 and 2) is considered a surrogate marker for a decreased in vivo CFTR chloride channel function. We, therefore, correlated the changes in FGF19 and C4 of patients with a G551D mutation to the ivacaftor-induced decrease in sweat chloride levels (Fig.3a,b). There was no significant correlation between the change in either FGF19 or C4 and the improvement in sweat chloride levels after ivacaftor treatment $(r=-0.14, P=.19$ and $r=0.11, P=.28$, respectively).

To determine whether the difference in BA homeostasis upon ivacaftor treatment was related to improvement in clinical outcome parameters of CF, we correlated FGF19 and $\mathrm{C} 4$ level changes to ivacaftor induced changes in FEV1 (Fig. 3c,d). No significant 
Figure 3. Correlation between BA metabolism markers and sweat chloride level and FEV1\% of predicted

a.

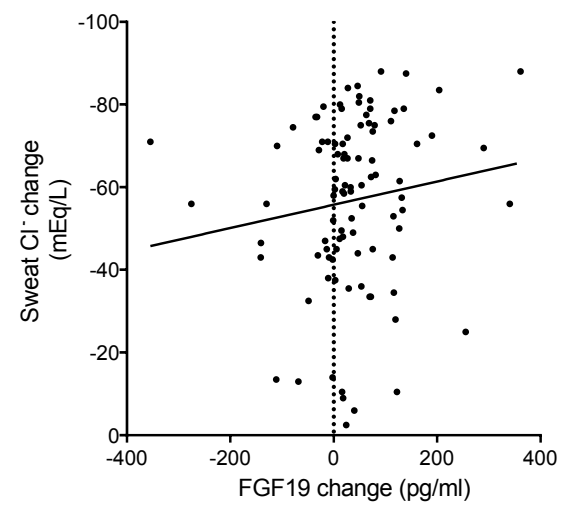

c.

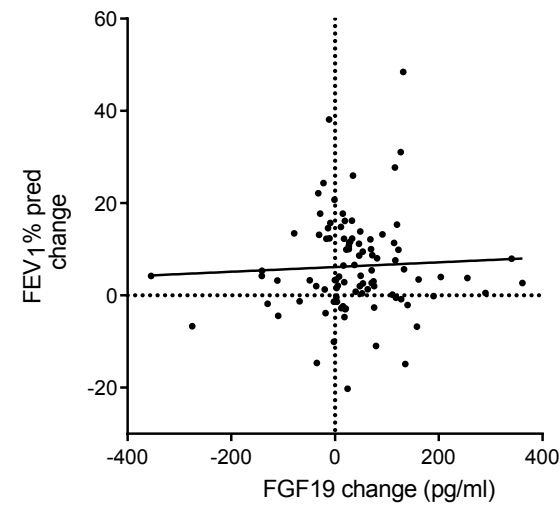

b.

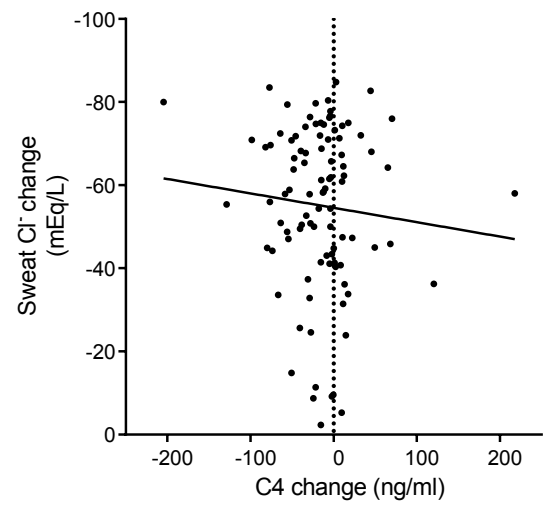

d.

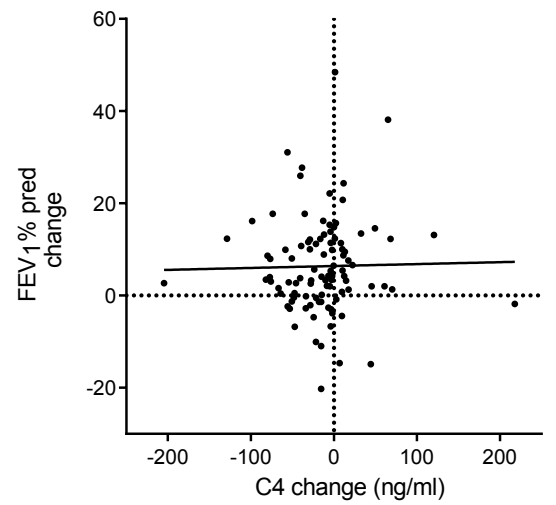

$(\mathbf{a}, \mathbf{b})$ an increase in FGF19 (Pearson $r=-0.14, P=.19$ ) and decrease in C4 (Pearson $r=0.11, P=.28$ ) are not significantly correlated with a greater decrease in sweat chloride level $n=96$;

(c,d) an increase in FGF19 (Pearson $r=0.05, P=.63$ ) and decrease in C4 (Pearson $r=0.02, P=.85$ ) are not significantly correlated with an increase in FEV $1 \%$ of predicted, $n=97$. 
correlation was found between either FGF19 ( $r=0.05, P=.63)$ or $C 4(r=0.02, P=.85)$ and FEV1. Similarly, no significant correlation was found between FGF19 or C4 and BMI (supplemental Fig. 1).

\section{DISCUSSION}

In this study, we demonstrate, in two independent studies, that the enterohepatic feedback regulation of BAs is impaired in CF patients with a class III CFTR gating mutation as reflected by decreased plasma FGF19 and increased plasma C4 levels compared to control values. Our data also demonstrate that ivacaftor treatment restored these markers towards normalization.

To establish baseline disturbances in FGF19 and C4 we compared CF values to healthy controls. We found that FGF19 levels were lower and C4 levels higher in both CF patients with an S1251 N or a G551D mutation, in line with a disruption of BA homeostasis. Unfortunately, we could not obtain control samples of age and BMI matched patients. However, aside from a surge of FGF19 in very early life (2-12 months of age) to our knowledge, there is no correlation with FGF19 and C4 and age. ${ }^{29}$ Additionally, the median C4 level in our control group was $14 \mathrm{ng} / \mathrm{ml}$ which corresponds to healthy control values reported in the literature, ranging from 9 to $19 \mathrm{ng} / \mathrm{ml} .{ }^{30}$ To confirm that age did not play a role in our study we performed a sub-analysis of FGF19 and C4 in different age groups and did not find a significant difference (data not shown). As for BMI, it has been shown that overweight and obesity correlate with lower plasma FGF19 levels. ${ }^{31}$ As the median BMI in our control group was 24.4 (Table 1 and 2), control FGF19 values could represent a slight underestimation compared to a lower BMI controlled group. However, we found no correlation between BMI and FGF19 levels in our control group nor in CF patients (data not shown). Additionally, BMI values in children under 18 are dependent on age and could, therefore, represent an underestimation compared to similar values in adults. ${ }^{32}$

Even though ivacaftor treatment significantly improved median FGF19 and C4 levels, there was a large variation between patients. We have identified several factors which may contribute to the variation. First, plasma FGF19 and C4 levels both express a diurnal rhythm and respond to food intake. ${ }^{10}$ Postprandial FGF19 levels are known to rise about 2-fold within 1-3 h after having a meal and are also differentially affected by carbohydrates, lipids, and proteins. ${ }^{33}$ Especially for C4 diurnal variation is pronounced with two peaks of a 2 to 4 fold increase during the day. ${ }^{34}$ For the G551D and S1251N study patients were not fasted and the time at which blood samples were taken was not standardized. This likely added more variation to the measured parameters and could account for the small portion of patients for which FGF19 and C4 changed in the opposite direction. However, on group level, we observed a similar change in FGF19 and C4 in two 
independent cohorts supporting our conclusion that parameters of bile acid metabolism improve on ivacaftor treatment in CF patients with specific mutations. However it remains possible that the observed beneficial effects would be more pronounced if obtained after fasting and under more standardized conditions.

These results elicit speculation on the exact mechanism underlying the effects of ivacaftor on the enterohepatic circulation of BAs. There could be a direct effect of potentiated CFTR on ileal BA uptake or on intestinal FXR activation. CFTR has previously been implicated in modulating the expression and in enhancing the function of the apical sodium bile acid transporter (ASBT), the main BA uptake transporter in the distal small intestine. ${ }^{5}$ Alternatively, the effect of ivacaftor could be indirect, for example on intestinal factors that could modulate BA metabolism such as BA absorption, inflammation or microbial composition. Absorption might also be increased by an alteration in the intestinal luminal mucus layer resulting in higher permeability for BAs towards the apical membrane of the enterocyte. Ivacaftor could ameliorate intestinal inflammation or dysbiosis, common features of the CF gastro-intestinal phenotype. ${ }^{9,35,36}$

The relevance of an improved enterohepatic BA circulation is not limited to possible improvement of nutrient (particularly fat) absorption. Recent studies suggest an important role of BA homeostasis in various metabolic processes. ${ }^{37}$ In addition, FGF19 has hormone-like properties and is implicated to beneficially affect glucose homeostasis, metabolic syndrome, and liver regeneration. ${ }^{38,39}$ Since CF patients are prone to develop metabolic complications, an improved bile acid homeostasis could potentially ameliorate these disorders. ${ }^{40,41}$

The improvements in plasma FGF19 and C4 levels did not correlate with the improvement in sweat chloride levels. This suggests that the ivacaftor-induced improvement in CFTR function is quantitatively different in vivo between various organ systems (e.g. sweat gland and Gl system). ${ }^{42}$ However, a recent study in CF patients with a G551D mutation found that there is a high rate of inter-and intra-subject variability in sweat chloride levels which could also account for some of the discrepancy.43 Another factor that could contribute to the difference is that ivacaftor is taken orally and results in a higher bioavailability in the intestine compared to other organs. The effect could, therefore, be more potent in the intestine. A recent study supports this idea by showing that the combination of lumacaftor/ivacaftor treatment in CF patients with a Phe508del mutation showed greater improvements in vivo intestinal CFTR function (assessed by intestinal current measurement) compared to respiratory epithelial CFTR function (measured by nasal potential difference). ${ }^{44}$ In this study there was also no correlation between these parameters and other CF-related outcomes (FEV1, sweat chloride and BMI) supporting the notion of heterogeneity in the individual responses to CFTR correction therapies. Additionally, CFTR potentiation in the intestine could affect various other Gl factors that are disturbed in CF and influence BA metabolism directly or indirectly. 
These include effects on intestinal $\mathrm{pH}$, microbiota, SIBO and inflammation. Ivacaftor has also been shown to affect other ATP-binding cassette (ABC) transporters such as ABCB4 (MDR3), which mediates phosphatidylcholine secretion into the bile. ${ }^{45,46} \mathrm{BMI}$ in CF is mainly dependent on pancreatic insufficiency and the pulmonary condition.47 Changes in BMI were not correlated to the changes in BA homeostasis. However, BMI is not only a representation of nutritional status or the functionality of the Gl tract but is also related to whole body metabolism. ${ }^{48} \mathrm{FEV} 1$ is a reflection of pulmonary function and dependent on many variables including pulmonary infection, inflammation, smoking, and exercise. Also, when baseline FEV1 level is low, there is usually some irreversible damage present making treatment induced improvement more tedious. It is known that there is a relatively large variation in the FEV1 improvement of individual patients treated with ivacaftor. The large inter-individual variation might corroborate the correlation between FEV1 and parameters of BA homeostasis.

These observations indicate that the effect of CFTR modulation, potentially, varies in different organ systems, e.g. in the pulmonary system vs. the gastrointestinal tract. Different individual outcome parameters such as FEV1, BMI and sweat chloride concentration, may differ in the dependency on direct CFTR protein function. There could also be a variation in the involvement of gene and environmental protein function modifiers in the various organ systems. Based on these considerations, we conclude that it is sensible to use an extended panel of available outcomes measures in different organ systems to access the overall therapeutic effects of CFTR modulators. ${ }^{49}$

\section{CONCLUSION}

In conclusion, our data show that FGF19 and C4 provide information on the disruption of BA homeostasis in CF and their improvement upon CFTR modulation therapy. Since FGF19 and C4 can be measured in plasma, they are relatively easy to obtain and can be used even in young children and neonates with still preserved growth and lung function. ${ }^{50}$ However additional studies are indicated to investigate the relationship between FGF19 and $\mathrm{C} 4$ and parameters of intestinal and liver function. Additionally, research is needed to validate FGF19 and C4 as potential biomarkers for clinical trials in CF. 


\section{ACKNOWLEDGEMENTS}

The authors would like to thank Claude P. van der Ley and Martijn Koehorst (Department of Laboratory Medicine, University Medical Center Groningen) for performing the C4 and bile acid measurements in plasma. This study was funded by grants from the Stichting Beatrix Kinderziekenhuis, the Dutch Cystic Fibrosis Foundation (NCFS), and the Jan Cornelis de Cock Stichting. We thank CFFT (Cystic Fibrosis Foundation Therapeutics) for providing the samples and the patient data. Special thank belongs to Steven M. Rowe, M.D. M.S.P.H. as the Sponsor-Investigator for the GOAL study along with the GOAL Investigators of the Cystic Fibrosis Foundation Therapeutics Development Network. The authors thank all participants in the LifeLines Cohort Study.

Funding: This work was supported by grants from the Stichting Beatrix Kinderziekenhuis, the Dutch Cystic Fibrosis Foundation (NCFS), the Jan Cornelis de Cock Stichting, and NWOZonMW (PTO project \#95104014). 


\section{REFERENCES}

1. Ratjen F, Döring G. Cystic fibrosis. Lancet. 2003;361(9358):681-689.

2. Gelfond D, Borowitz D. Gastrointestinal Complications of Cystic Fibrosis. Clin Gastroenterol Hepatol. 2013;11(4):333-342.

3. Kerem B, Rommens J, Buchanan J, et al. Identification of the cystic fibrosis gene: genetic analysis. Science (80- ). 1989;245(4922):1073-1080.

4. O'Brien S, Mulcahy H, Fenlon $\mathrm{H}$, et al. Intestinal bile acid malabsorption in cystic fibrosis. Gut. 1993;34(8):1137-1141.

5. Bijvelds MJC, Jorna H, Verkade HJ, et al. Activation of CFTR by ASBT-mediated bile salt absorption. Am J Physiol Gastrointest Liver Physiol. 2005;289(5):G870-9.

6. Strandvik B, Einarsson K, Lindblad A, Angelin B. Bile acid kinetics and biliary lipid composition in cystic fibrosis. J Hepatol. 1996;25(1):43-48.

7. Kuipers F, Bloks VW, Groen AK. Beyond intestinal soap-bile acids in metabolic control. Nat Rev Endocrinol. 2014;10(8):488-498.

8. Ooi CY, Durie PR. Cystic fibrosis from the gastroenterologist's perspective. Nat Rev Gastroenterol Hepatol. 2016;13(3):175-185.

9. Ridlon JM, Kang D-J, Hylemon PB. Bile salt biotransformations by human intestinal bacteria. J Lipid Res. 2006;47(2):241-259.

10. Lundåsen T, Gälman C, Angelin B, Rudling M. Circulating intestinal fibroblast growth factor 19 has a pronounced diurnal variation and modulates hepatic bile acid synthesis in man. $J$ Intern Med. 2006;260(6):530-536.

11. Chiang JYL. Bile acids: regulation of synthesis. J Lipid Res. 2009;50(10):1955-1966.

12. Gälman C, Arvidsson I, Angelin B, Rudling M. Monitoring hepatic cholesterol 7 -hydroxylase activity by assay of the stable bile acid intermediate 7 -hydroxy-4-cholesten-3-one in peripheral blood. J Lipid Res. 2003;44(4):859-866.

13. Walters JRF, Johnston IM, Nolan JD, Vassie C, Pruzanski ME, Shapiro DA. The response of patients with bile acid diarrhoea to the farnesoid $\mathrm{X}$ receptor agonist obeticholic acid. Aliment Pharmacol Ther. 2015;41(1):54-64.

14. Pattni SS, Brydon WG, Dew T, et al. Fibroblast growth factor 19 in patients with bile acid diarrhoea: a prospective comparison of FGF19 serum assay and SeHCAT retention. Aliment Pharmacol Ther. 2013;38(8):967-976.

15. Aziz I, Mumtaz S, Bholah H, Chowdhury FU, Sanders DS, Ford AC. High Prevalence of Idiopathic Bile Acid Diarrhea Among Patients With Diarrhea-Predominant Irritable Bowel Syndrome Based on Rome III Criteria. Clin Gastroenterol Hepatol. 2015;13(9):1650-1655.e2.

16. Wong BS, Camilleri M, Carlson P, et al. Increased Bile Acid Biosynthesis Is Associated With Irritable Bowel Syndrome With Diarrhea. Clin Gastroenterol Hepatol. 2012;10(9):1009-1015. e3.

17. Accurso FJ, Rowe SM, Clancy JP, et al. Effect of VX-770 in persons with cystic fibrosis and the G551D- CFTR mutation. N Engl J Med. 2010;363(21):1991-2003.

18. Yu H, Burton B, Huang C-J, et al. Ivacaftor potentiation of multiple CFTR channels with gating mutations. J Cyst Fibros. 2012;11(3):237-245.

19. Noordhoek-van der Staay JJ, Gulmans VAM. Dutch CF Registry, 2016.; 2017.

20. Cystic Fibrosis Foundation. Cystic Fibrosis Foundation Patient Registry 2014 Annual Data Report. Bethesda, Maryland; 2016.

21. Ramsey BW, Davies J, McElvaney NG, et al. A CFTR potentiator in patients with cystic fibrosis and the G551D mutation. N Engl J Med. 2011;365(18):1663-1672.

22. Davies JC, Wainwright CE, Canny GJ, et al. Efficacy and safety of ivacaftor in patients aged 6 to 11 years with cystic fibrosis with a G551D mutation. Am J Respir Crit Care Med. 2013;187(11):1219-1225. 
23. De Boeck K, Munck A, Walker S, et al. Efficacy and safety of ivacaftor in patients with cystic fibrosis and a non-G551D gating mutation. J Cyst Fibros. 2014;13(6):674-680.

24. Gelfond D, Heltshe S, Ma C, et al. Impact of CFTR Modulation on Intestinal pH, Motility, and Clinical Outcomes in Patients With Cystic Fibrosis and the G551D Mutation. Clin Transl Gastroenterol. 2017;8(3):e81.

25. Rowe SM, Heltshe SL, Gonska T, et al. Clinical Mechanism of the Cystic Fibrosis Transmembrane Conductance Regulator Potentiator Ivacaftor in G551D-mediated Cystic Fibrosis. Am J Respir Crit Care Med. 2014;190(2):175-184.

26. Farrell PM, Rosenstein BJ, White TB, et al. Guidelines for Diagnosis of Cystic Fibrosis in Newborns through Older Adults: Cystic Fibrosis Foundation Consensus Report. J Pediatr. 2008;153(2):S4-S14.

27. Scholtens S, Smidt N, Swertz MA, et al. Cohort Profile: LifeLines, a three-generation cohort study and biobank. Int J Epidemiol. 2015;44(4):1172-1180.

28. Camilleri M, Busciglio I, Acosta A, et al. Effect of Increased Bile Acid Synthesis or Fecal Excretion in Irritable Bowel Syndrome-Diarrhea. Am J Gastroenterol. 2014;109(10):16211630.

29. Sánchez-Infantes D, Gallego-Escuredo JM, Díaz M, et al. Circulating FGF19 and FGF21 surge in early infancy from infra- to supra-adult concentrations. Int J Obes (Lond). 2015;39(5):742-746.

30. Freudenberg F, Gothe F, Beigel F, Rust C, Koletzko S. Serum 7-Alpha-Hydroxy-4Cholesten-3-One as a Marker for Bile Acid Loss in Children. J Pediatr. 2013;163(5):13671371.e1.

31. Gallego-Escuredo JM, Gómez-Ambrosi J, Catalan V, et al. Opposite alterations in FGF21 and FGF19 levels and disturbed expression of the receptor machinery for endocrine FGFs in obese patients. Int J Obes. 2015;39(1):121-129.

32. Maynard LM, Wisemandle W, Roche AF, Chumlea WC, Guo SS, Siervogel RM. Childhood Body Composition in Relation to Body Mass Index. Pediatrics. 2001;107(2):344-350.

33. Morton GJ, Kaiyala KJ, Foster-Schubert KE, Cummings DE, Schwartz MW. Carbohydrate Feeding Dissociates the Postprandial FGF19 Response From Circulating Bile Acid Levels in Humans. J Clin Endocrinol Metab. 2014;99(2):E241-E245.

34. Gälman C, Angelin B, Rudling M. Bile Acid Synthesis in Humans Has a Rapid Diurnal Variation That Is Asynchronous With Cholesterol Synthesis. Gastroenterology. 2005;129(5):1445-1453.

35. Werlin SL, Benuri-Silbiger I, Kerem E, et al. Evidence of Intestinal Inflammation in Patients With Cystic Fibrosis. J Pediatr Gastroenterol Nutr. 2010;51(3):1.

36. Norkina O, Burnett TG, De Lisle RC. Bacterial Overgrowth in the Cystic Fibrosis Transmembrane Conductance Regulator Null Mouse Small Intestine. Infect Immun. 2004;72(10):6040-6049.

37. Lefebvre P, Cariou B, Lien F, Kuipers F, Staels B. Role of Bile Acids and Bile Acid Receptors in Metabolic Regulation. Physiol Rev. 2009;89(1):147-191.

38. Wu A-L, Coulter S, Liddle C, et al. FGF19 Regulates Cell Proliferation, Glucose and Bile Acid Metabolism via FGFR4-Dependent and Independent Pathways. Gaetano C, ed. PLoS One. 2011;6(3):e17868.

39. Naugler WE, Tarlow BD, Fedorov LM, et al. Fibroblast Growth Factor Signaling Controls Liver Size in Mice With Humanized Livers. Gastroenterology. 2015;149(3):728-740.e15.

40. Moyer K, Balistreri W. Hepatobiliary disease in patients with cystic fibrosis. Curr Opin Gastroenterol. 2009;25(3):272-278.

41. Kelly A, Moran A. Update on cystic fibrosis-related diabetes. J Cyst Fibros. 2013;12(4):318331.

42. Durmowicz AG, Lim R, Rogers H, Rosebraugh CJ, Chowdhury BA. The U.S. Food and Drug Administration's Experience with Ivacaftor in Cystic Fibrosis. Establishing Efficacy Using In vitro Data in Lieu of a Clinical Trial. Ann Am Thorac Soc. 2018;15(1):1-2. 
43. Vermeulen F, Le Camus C, Davies JC, Bilton D, Milenković D, De Boeck K. Variability of sweat chloride concentration in subjects with cystic fibrosis and G551D mutations. J Cyst Fibros. 2017;16(1):36-40.

44. Graeber SY, Hug MJ, Sommerburg O, et al. Intestinal Current Measurements Detect Activation of Mutant CFTR in Patients with Cystic Fibrosis with the G551D Mutation Treated with Ivacaftor. Am J Respir Crit Care Med. 2015;192(10):1252-1255.

45. Vauthier $V$, Housset C, Falguières T. Targeted pharmacotherapies for defective ABC transporters. Biochem Pharmacol. 2017;136:1-11.

46. Delaunay J-L, Bruneau A, Hoffmann B, et al. Functional defect of variants in the adenosine triphosphate-binding sites of ABCB4 and their rescue by the cystic fibrosis transmembrane conductance regulator potentiator, ivacaftor (VX-770). Hepatology. 2017;65(2):560-570.

47. Stephenson AL, Mannik LA, Walsh S, et al. Longitudinal trends in nutritional status and the relation between lung function and BMI in cystic fibrosis: a population-based cohort study. Am J Clin Nutr. 2013;97(4):872-877.

48. Bederman I, Perez A, Henderson L, et al. Altered de novo lipogenesis contributes to low adipose stores in cystic fibrosis mice. Am J Physiol Liver Physiol. 2012;303(4):G507-G518.

49. Bodewes FAJA, Verkade HJ, Taminiau JAJM, Borowitz D, Wilschanski M. Cystic fibrosis and the role of gastrointestinal outcome measures in the new era of therapeutic CFTR modulation. J Cyst Fibros. 2015;14(2):169-177.

50. Memon N, Griffin IJ, Lee CW, et al. Developmental regulation of the gut-liver (FGF19CYP7A1) axis in neonates. J Matern Neonatal Med. October 2018:1-6. 


\section{SUPPLEMENTALS}

Supplemental Table S1. Profile of individual BA species in percentage before and 2 months after treatment with ivacaftor in $\mathrm{S} 1251 \mathrm{~N}$ patients

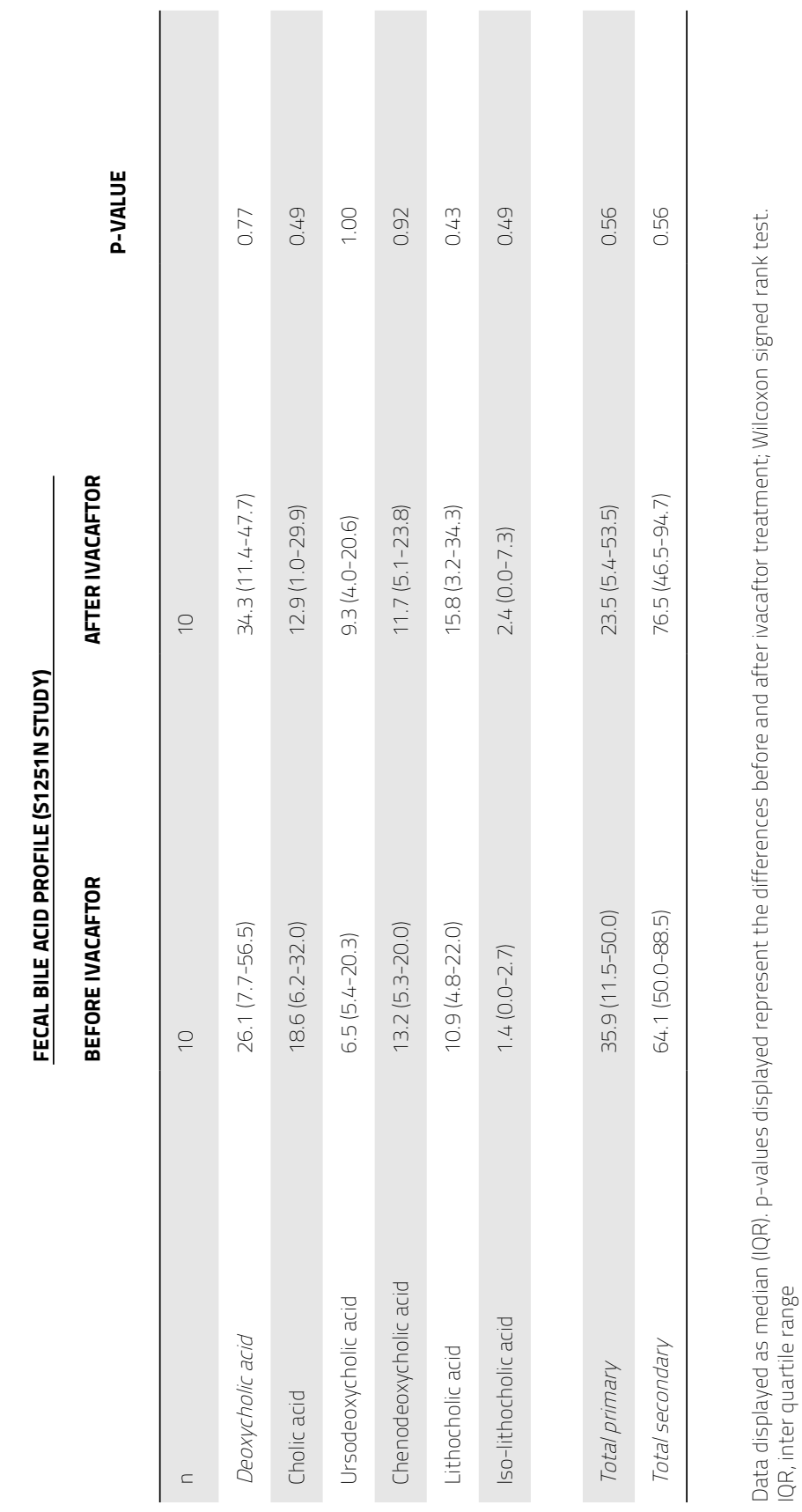


Supplemental Table S2. Concentration and profile of individual BA species in plasma before and 2 months after treatment with ivacaftor in S1251N patients

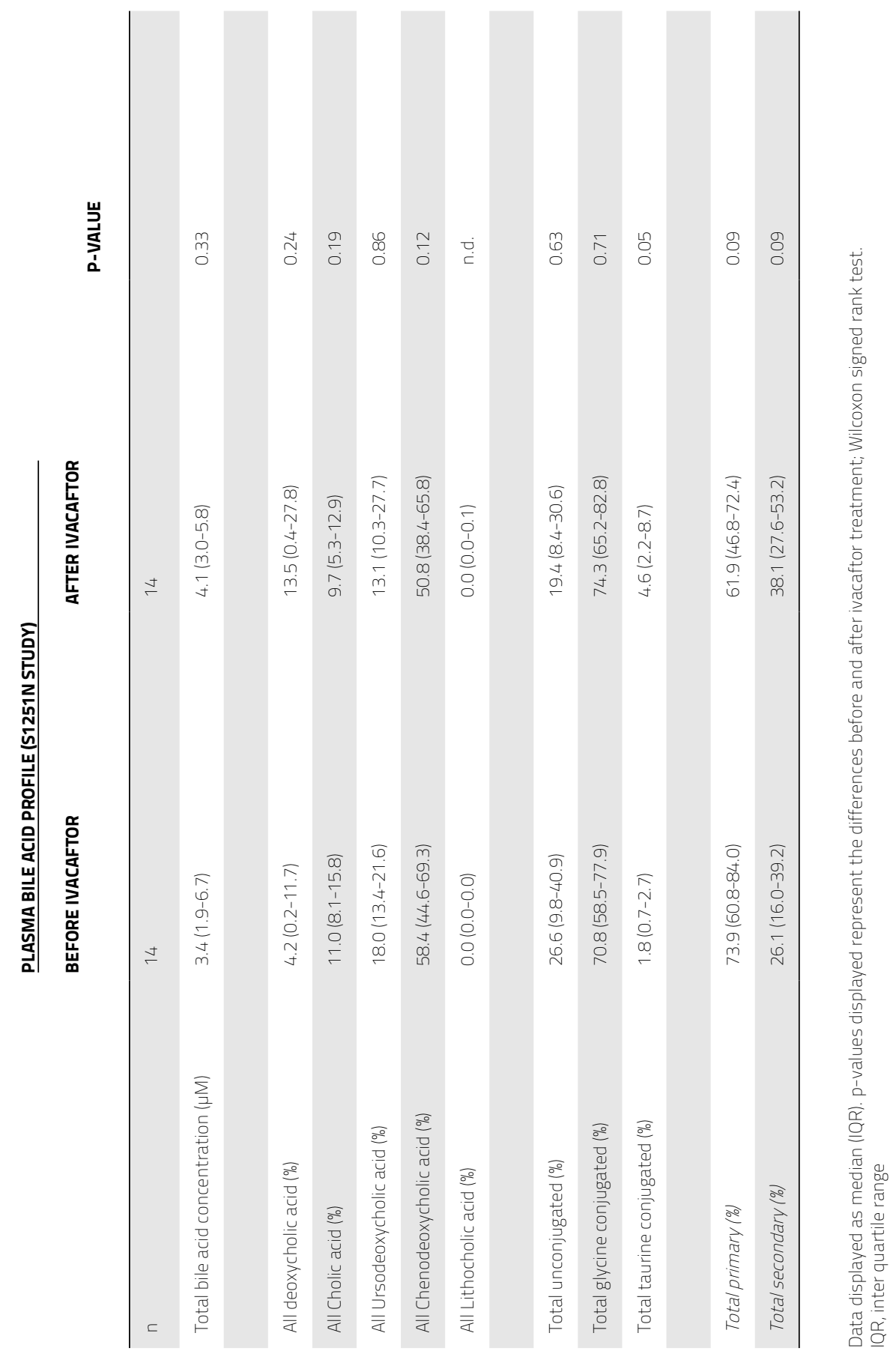


Supplemental Figure S1. Correlation between BA metabolism markers and BMI in G551D study

a.

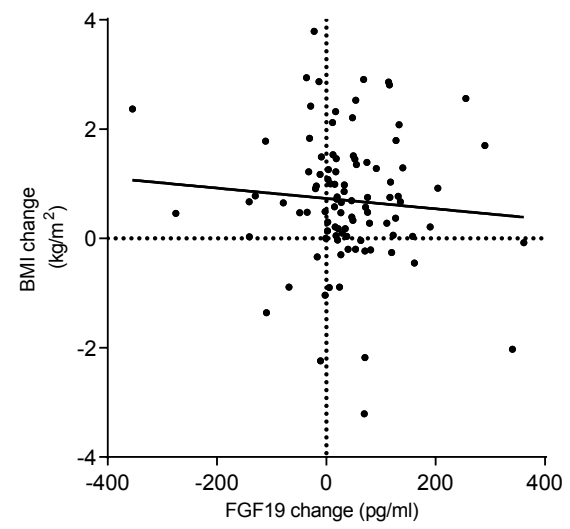

b.

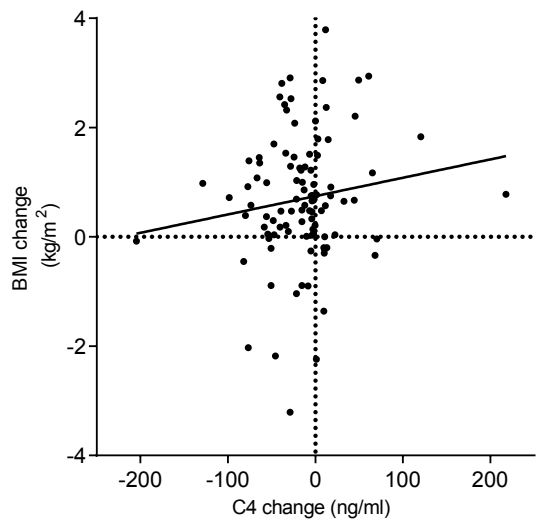

(a) Correlation between change in FGF19 (Pearson $r=-0.08$, P $=0.42$ ) and change in BMl after treatment with ivacaftor in G551D patients, $n=99$.

(b) Correlation between change in C4 (Pearson $r=0.14, \mathrm{P}=0.17$ ) and change in BMI after treatment with ivacaftor in G551D patients, $n=99$ 


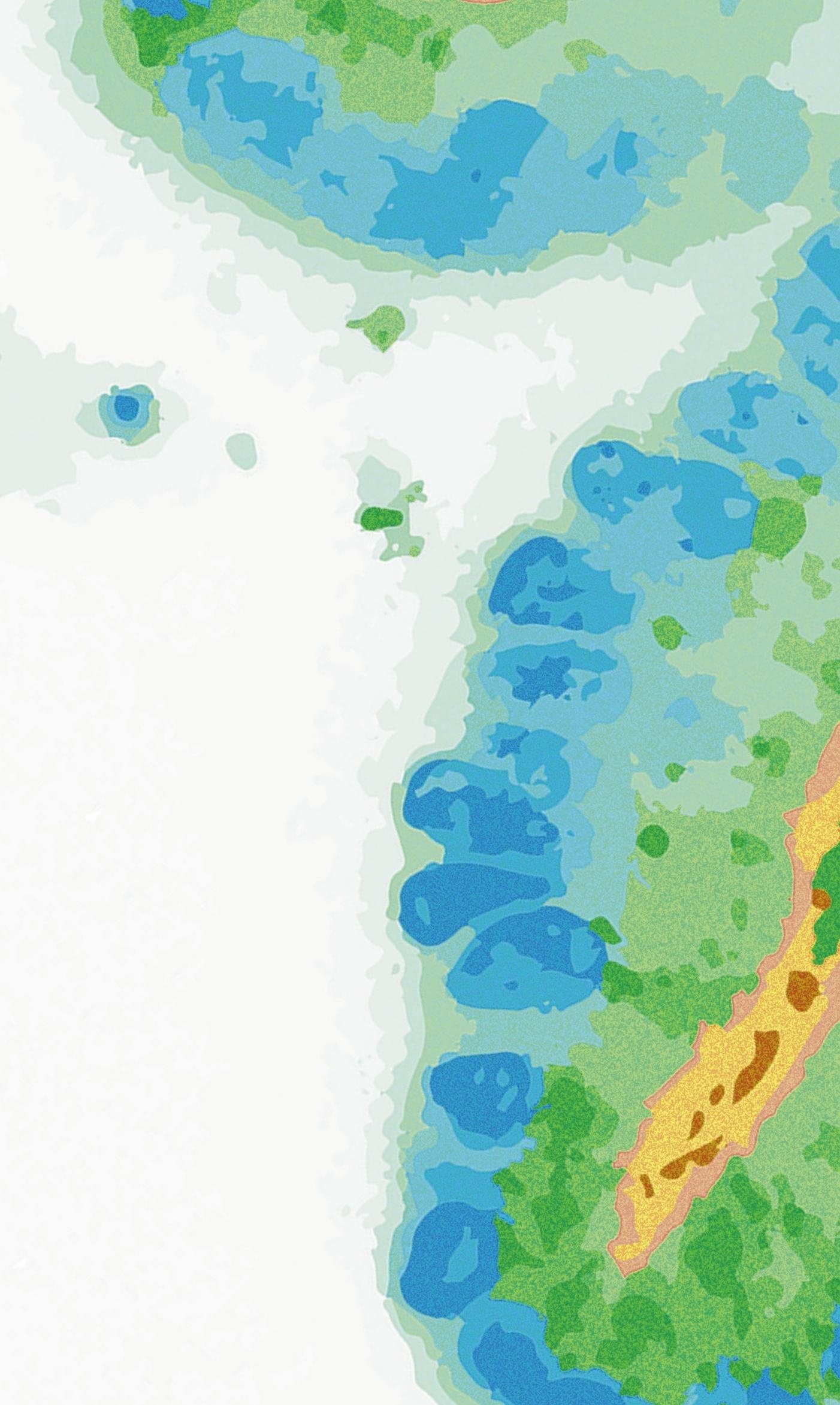




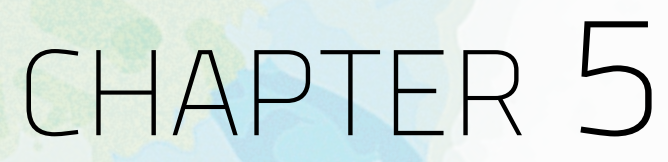

\section{A PHASE 2 CROSSOVER STUDY OF LUMACAFTOR / IVACAFTOR IN CYSTIC FIBROSIS PATIENTS WITH AN A455E-CFTR MUTATION}




\section{ABSTRACT}

Background: p.Ala455Glu (A455E), a rare mutation in the cystic fibrosis transmembrane conductance regulator gene (CFTR), has a prevalence of $4.1 \%$ in the Netherlands. Based on previous data showing that A455E-CFTR responds to lumacaftor/ivacaftor (LUM/IVA) in vitro, this study was conducted to explore the clinical efficacy of LUM/IVA in patients with cystic fibrosis who had at least one A455E-CFTR mutation.

Methods: This was a small, exploratory, phase 2, crossover study (NCT03061331). Patients aged $\geq 12$ years were randomized to 1 of 2 treatment sequences (LUM/IVA -> placebo or placebo -> LUM/IVA). Patients were treated for 8 weeks with LUM/IVA and 8 weeks with placebo with an 8-week washout period in between. Primary endpoint was absolute change in ppFEV1 from study baseline through 8 weeks.

Results: Twenty patients were randomized at 2 sites in the Netherlands. The mean absolute change in ppFEV1 from study baseline through week 8 showed a treatment difference of 0.1 percentage points $(95 \% \mathrm{Cl},-2.5$ to 2.7 ; $\mathrm{P}=0.928)$ between LUM/IVA (within-group change, 2.7) and placebo (within-group change, 2.6). The mean absolute change in sweat chloride concentration from study baseline through week 8 showed a treatment difference of $-7.8 \mathrm{mmol} / \mathrm{L}$ between LUM/IVA and placebo $(P=0.004)$, while the absolute change in CFQ-r respiratory domain score showed a treatment difference of 3.5 between LUM/IVA and placebo $(P=0.469)$. No new unexpected adverse events were identified with LUM/IVA therapy.

Conclusions: Although the study did not meet its primary endpoint, all other endpoints showed a trend towards a potential clinical benefit with LUM/IVA in patients with a A455E-CFTR mutation. 


\section{INTRODUCTION}

Cystic fibrosis (CF) results from mutations in the cystic fibrosis transmembrane conductance regulator (CFTR) gene that reduce the quantity and/or function of the CFTR protein, which is an epithelial chloride channel that regulates chloride transport across epithelia in exocrine organs including the lung and pancreas.1 Progressive lung function decline is the leading cause of mortality among patients with CF.2,3 The p.Ala455Glu (A455E) is a Class $V$ mutation that generates CFTR protein with a shortened halflife due to rapid degradation, resulting in a reduction of mature CFTR protein; in vitro studies suggested that the quantity of functional protein at the cell surface is $12 \%$ of the normal CFTR amount. ${ }^{4-7}$ Worldwide, A445E mutations have been reported in $<0.1 \%$ of patients with $C F$, although the prevalence varies by region. ${ }^{8,9}$ In the Netherlands, the A $455 E$ mutation occurs in $4.1 \%$ of patients with CF, with an even greater prevalence reported in the Southeast and Southwest regions of the country. ${ }^{9,10}$ Clinical experience with the A455E mutation, initially associated with a less severe CF phenotype, has shown differences in disease severity by young adulthood, ranging from less severe to severe loss of lung function. 8,11,12 Patients with an A455E mutation are usually diagnosed later than their counterparts homozygous for the F508del mutation, although newborn screening initiatives have helped identify patients earlier. ${ }^{8,11-13}$ Nevertheless, patients with residual function mutations such as A455E demonstrate clinical characteristics of CF lung disease, including declining lung function-albeit slower compared with those homozygous for F508del — that is more rapid in adolescents and young adults. ${ }^{14}$

Ivacaftor (IVA or VX-770) is a CFTR potentiator that increases the channel open probability of CFTR at the cell surface. Lumacaftor (LUM or VX-809) and tezacaftor are CFTR correctors that increase the quantity of CFTR delivered to the cell surface; these are combined with a CFTR potentiator for their synergistic effects. Combined lumacaftor/ ivacaftor (LUM/IVA) therapy improves lung function and provides multisystemic clinical benefits in patients with CF who are homozygous for the F508del mutation, a mutation that results in processing and trafficking defects. ${ }^{15}$ Improvements in forced expiratory volume in 1 second (FEV1) were observed as early as Day 15 in patients $\geq 12$ years on LUM/IVA compared to those on placebo and were sustained through 24 weeks of treatment in the pivotal phase 3 studies (TRAFFIC and TRANSPORT). ${ }^{15}$ Additional studies of LUM/IVA have led to approval in patients as young as 2 years of age with CF who are homozygous for the F508del mutation. ${ }^{16,17}$

Recently, a novel CFTR functional assay using cultures of intestinal stem cells, referred to as organoids, was developed. ${ }^{18}$ Organoids derived from healthy controls swell in response to forskolin-induced activation of CFTR-dependent chloride secretion. Forskolin-induced swelling is reduced in organoids derived from patients homozygous for the F508del mutation compared with those from healthy controls and can be restored by incubation of the organoids with VX-809/VX-770. VX-809/VX-770-induced improvement of 
swelling of organoids was also observed in A455E/F508del organoids. ${ }^{19}$ In vitro data thus supports the use of similar correction and potentiation strategies for the treatment of the A445E and F508del mutations, including treatment with LUM/IVA. ${ }^{19}$ Based on these pre-clinical data, this study was designed to explore the efficacy of LUM/IVA in patients with CF who had at least one A455E-CFTR mutation.

\section{METHODS}

\section{Study design and patients}

This was a randomized, double-blind, placebo-controlled, multicenter, phase 2 crossover study that took place in the Netherlands (VX15-809-111; NCT03061331). It included two 8-week treatment periods separated by an 8-week ( \pm 7 days) washout period (Fig. 1a). Treatment period 1 was from day 1 to week 8 and treatment period 2 was from week 16 to week 24. Twenty patients were randomized 1:1 to receive the 2 treatment sequences. In treatment sequence 1, patients received LUM/IVA in treatment period 1 and placebo in treatment period 2 (LUM/IVA-P). In treatment sequence 2 , patients received placebo in treatment period 1 and LUM/IVA in treatment period 2 (P-LUM/IVA). The approved dose of LUM/IVA (LUM 400 mg/IVA 250 mg every 12 hours (q12h)) or matching placebo q12h were given orally. An 8-week washout period between the two treatment periods was chosen based on the terminal half-lives of LUM (26 hours) and IVA (12 hours) and on previous clinical study results. ${ }^{16,17,20}$

Evaluating drugs intended for rare disease populations poses challenges in clinical trial design due to small sample sizes and patient population heterogeneity. Guidelines were developed by the international rare diseases research consortium to accelerate research for rare diseases. ${ }^{21}$ These guidelines suggest alternative strategies (e.g., crossover and n-of-1 trials) to maximize efficiency and minimize risk of bias when randomized parallel trial designs are not feasible. ${ }^{21,22}$ Given the limited patient population available, a crossover design was chosen that enabled treatment of the same patient with both placebo and LUM/IVA in different treatment periods. The use of a double-blind design reduced the chance of bias. Patients with stable CF who were $\geq 12$ years of age with at least 1 A455ECFTR mutation and a ppFEV1 of $\geq 30 \%$ and $\leq 90 \%$ were eligible for this study. This study was conducted in accordance with the international council for harmonisation good clinical practice (ICH GCP) guidelines, consistent with the principles of the declaration of Helsinki. Study documentation was approved by institutional ethics committees for each study site. All patients (and/or their legal guardian) provided written informed consent.

\section{Objective and outcomes}

In this exploratory study, the clinical response to LUM/IVA in patients $\geq 12$ years of 
age with CF with the A455E-CFTR mutation was investigated. The primary endpoint was absolute change in ppFEV1 from study baseline through 8 weeks of treatment of either treatment period, calculated using the 2012 global lung initiative equations. ${ }^{23}$ Other endpoints included the absolute change in sweat chloride concentration from study baseline through 8 weeks of treatment and absolute change in cystic fibrosis questionnaire-revised (CFQ-r) respiratory domain score from study baseline at the end of 8 weeks of treatment of either period.

\section{Statistical analysis}

Because the A455E-CFTR mutation is so rare, no formal sample size calculations were conducted for this exploratory study. The planned sample size of 20 patients was based on the number of patients expected to be available and willing to participate.

For this crossover study, 2 different baselines were defined (Fig. 1a). Study baseline was defined as the most recent non-missing measurement (scheduled or unscheduled) collected prior to the first dose of study drug (either placebo or LUM/IVA) in the study. The definition was applied to all demographics, background, and baseline characteristics and also to data analyses, including the primary endpoint analysis. Period baseline was defined as the most recent non-missing measurement (scheduled or unscheduled) collected before the first dose of study drug in treatment period 1 or treatment period 2 . Absolute change from study and period baseline were calculated as post-baseline values minus the study and period baseline value, respectively.

The primary analysis for the primary efficacy endpoint, the absolute change in ppFEV from study baseline through 8 weeks of treatment of either treatment period, was based on a mixed effects model for repeated measures (MMRM). The model included the absolute change from the study baseline in each treatment period as the dependent variable, with sequence, treatment, period, visit within period, and treatment-by-visit interaction as fixed effects, study baseline ppFEV 1 as a covariate, and patient nested within sequence as the random effect. In the model, visit was treated as a class variable. An unstructured covariance matrix was assumed for the repeated measurements of the same patient within each treatment period. As a supportive sensitivity analysis, a similar pre-specified MMRM analysis was conducted for the changes in ppFEV $_{1}$ from period baseline. Similar analyses were done for the other endpoints (sweat chloride and CFQ-r respiratory domain), with the baseline of analyzed endpoint as the covariate. Difference between LUM/IVA and placebo through 8 weeks of treatment (defined as the average of 4 weeks and 8 weeks) were obtained from the MMRM models, estimated by least squares mean with a 2 sided $95 \% \mathrm{Cl}$ and a 2 sided $\mathrm{p}$-value. All reported $\mathrm{p}$-values for other endpoints are nominal $p$-values. All treatment-emergent adverse events ( $A E s$; defined as AEs that increased in severity or that were newly developed at or after the initial dose of study drug in a given treatment period to 28 days after the last dose of study 
drug in that treatment period (or safety follow-up visit, whichever is last)) were assessed, documented, and reported in accordance with ICH GCP guidelines.

\section{RESULTS}

\section{Patients}

Twenty patients were randomized 1:1 to receive the 2 treatment sequences at the 2 study sites. After randomization, patients continued their concomitant medications, most commonly for CF management (e.g., salbutamol, dornase alfa, and azithromycin). Overall, $60 \%$ of patients were female and the mean age was 38 years, with the majority $(90 \%)$ being $\geq 18$ years of age (Table 1). Ninety percent of patients enrolled in the study had an F508del-CFTR mutation on the second allele. Baseline mean ppFEV1 was 58.9 percentage points (range, 31.3 to 94.9) overall. All 20 randomized patients with the A455E-CFTR mutation received at least 1 dose of study drug and were included in the full analysis set and the safety set. All patients completed the 8 weeks dosing in treatment period 1 , and $17(85 \%)$ completed the 8 weeks dosing in treatment period 2 (Fig. 1 b). Three patients discontinued the study during the washout period due to AEs. All 3 AEs were infective pulmonary exacerbation of CF outside the treatment-emergent period, mild or moderate in severity, and deemed unrelated to the study drug. No patient discontinued during either treatment period.

\section{Efficacy}

The estimated mean absolute change in ppFEV1 from study baseline through 8 weeks of treatment (primary endpoint) showed a treatment difference of 0.1 percentage points (95\% confidence interval (CI), $-\neg 2.5$ to $2.7 ; \mathrm{P}=0.928$ ) between LUM/IVA and placebo (2.7 (standard error (SE), 1.1) percentage points change within LUM/IVA group vs $2.6(S E, 1.2)$ percentage points change within placebo group) (Fig. 2a). In the pre-specified supportive analysis, the estimated mean within-group absolute change in ppFEV1 from period baseline through 8 weeks was 3.2 (SE, 1.0) percentage points with LUM/IVA and 1.1 $(S E, 1.0)$ percentage points with placebo, which resulted in a treatment difference of 2.1 percentage points ( $95 \% \mathrm{Cl},-0.6$ to $4.8 ; \mathrm{P}=0.117$ ) (Table 2$)$.

The change in ppFEV1 from baseline was further assessed for treatment period 1 only, which was not subject to the impact of treatment crossover (Fig. 2b). In treatment period 1 , the estimated mean absolute change in ppFEV1 from period baseline through 8 weeks of treatment showed a treatment difference of 3.8 percentage points $(95 \% \mathrm{Cl}, 0.0$ to 7.7 ; $\mathrm{P}=0.049$ ) between the LUM/IVA group and the placebo group (3.9 (SE, 1.3) percentage points change within LUM/IVA group vs $0.0(S E, 1.3)$ percentage points change within placebo group). 
Table 1. Baseline patient demographics and characteristics

\begin{tabular}{|c|c|c|c|}
\hline & $\begin{array}{l}\text { LUM/IVA - P } \\
\text { (N=10) }\end{array}$ & $\begin{array}{l}P-\text { LUM/IVA } \\
(\mathrm{N}=10)\end{array}$ & $\begin{array}{l}\text { OVERALL } \\
(\mathrm{N}=20)\end{array}$ \\
\hline Female sex, $n(\%)$ & $7(70.0)$ & $5(50.0)$ & $12(60.0)$ \\
\hline Childbearing potential, $\cap(\%)$ a & $5(71.4)$ & $4(80.0)$ & $9(75.0)$ \\
\hline Mean age (range), years & $41.2(14-59)$ & $34.7(18-51)$ & $38.0(14-59)$ \\
\hline$\geq 12$ years to $<18$ years, $n(\%)$ & $2(20.0)$ & 0 & $2(10.0)$ \\
\hline$\geq 18$ years, $\mathrm{n}(\%)$ & $8(80.0)$ & $10(100.0)$ & $18(90.0)$ \\
\hline White race, $\mathrm{n}(\%)$ & $10(100.0)$ & $10(100.0)$ & $20(100.0)$ \\
\hline \multicolumn{4}{|l|}{ Mutation genotype, n (\%) } \\
\hline A455E/F508del & $9(90.0)$ & $9(90.0)$ & $18(90.0)$ \\
\hline A455E/otherb & $1(10.0)$ & $1(10.0)$ & $2(10.0)$ \\
\hline Mean weight (SD), kg & $64.8(12.8)$ & $72.6(11.5)$ & $68.7(12.5)$ \\
\hline Mean height (SD), cm & $170.3(6.9)$ & $178.2(8.4)$ & $174.3(8.5)$ \\
\hline Mean BMI (SD), kg/m2 & $22.3(3.5)$ & $22.9(3.5)$ & $22.6(3.4)$ \\
\hline \multicolumn{4}{|l|}{ ppFEV1, $\cap(\%)$} \\
\hline$<40 \%$ & 0 & $2(20.0)$ & $2(10.0)$ \\
\hline$\geq 40 \%$ to $<70 \%$ & $9(90.0)$ & $5(50.0)$ & $14(70.0)$ \\
\hline$\geq 70 \%$ to $\leq 90 \%$ & $1(10.0)$ & $2(20.0)$ & $3(15.0)$ \\
\hline$>90 \%$ & 0 & $1(10.0)$ & $1(5.0)$ \\
\hline Mean ppFEV1 (SD), percentage points & $57.7(9.4)$ & $60.0(20.0)$ & $58.9(15.3)$ \\
\hline Mean sweat chloride concentration (SD), mmol/L & $77.2(12.3)$ & $82.5(7.5)$ & $79.8(10.3)$ \\
\hline Mean CFQ-r respiratory domain score, (SD)c & $69.4(15.3)$ & $67.8(13.8)$ & $68.6(14.2)$ \\
\hline History of pancreatic insufficiency, $n(\%)$ & $2(20.0)$ & $1(10.0)$ & $3(15.0)$ \\
\hline
\end{tabular}

\footnotetext{
a Percentages for "childbearing potential" were calculated using the number of female patients as the denominator. b The 2 patients in the "other" mutation group had a Class I E60X mutation c Data from the CFQ-r "Ages 12 and 13" version and the "Adolescents and Adults" version were pooled together for analysis.

BMI, body mass index; CFQ-r, cystic fibrosis questionnaire-revised; cm, centimeter; kg, kilogram; L, liter; LUM/IVA-P, patients receiving lumacaftor/ivacaftor in treatment period 1 followed by placebo in treatment period $2 ; n$, number; P-LUM/IVA, patients receiving placebo in treatment period 1 followed by lumacaftor/ivacaftor in treatment period 2 pPFEV ${ }_{1}$, percent predicted forced expiratory volume in 1 second; SD, standard deviation.
} 
Figure 1. Study design and patient disposition

a

\section{Randomization \\ $(1: 1)$ \\ Treatment Sequence 1}

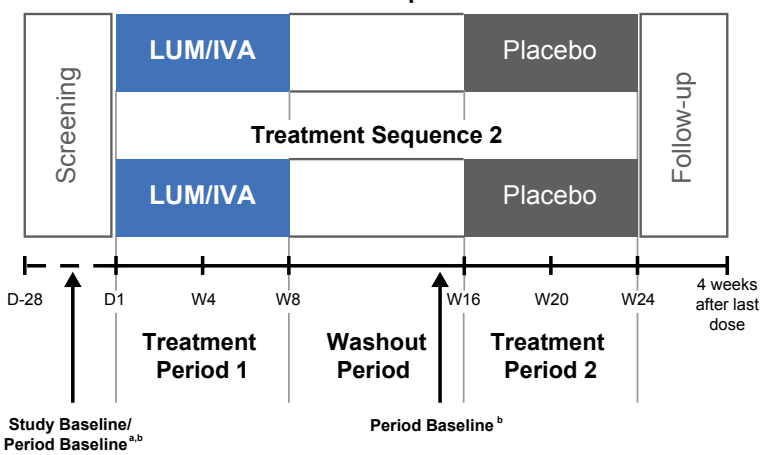

b

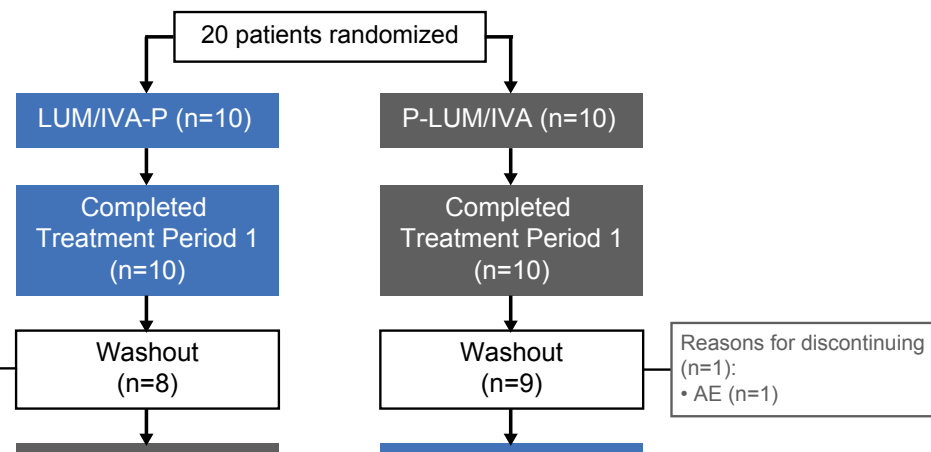

Completed

Treatment Period 2

$(n=8)$

Completed study

$(n=8)$
Completed

Treatment Period 2

$(n=9)$

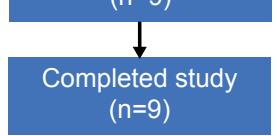

(a) In this phase 2, double-blind, placebo-controlled, crossover study eligible patients were randomized (1:1) to 1 of 2 treatment sequences (LUM/IVA followed by placebo (treatment sequence 1) or placebo followed by LUM/IVA (treatment sequence 2)) consisting of two 8-week treatment periods separated by an 8-week washout period,

(b) Overall, 20 patients were randomized; all received $\geq 1$ dose of study drug and completed treatment period 1. Of 10 patients randomized to treatment sequence 1 (LUM/IVA-P), 8 completed both treatment periods and 2 discontinued treatment during the washout period due to AEs. Of 10 patients randomized to treatment sequence 2 (P-LUM/IVA), 9 completed both treatment periods and 1 discontinued treatment during the washout period due to AEs

a Study baseline was the most recent non-missing measurement (scheduled or unscheduled) collected prior to the first dose of study drug (either placebo or LUM/IVA) in the study

b Period baseline was the most recent non-missing measurement (scheduled or unscheduled) collected before the first dose of study drug in treatment period 1 or treatment period 2

D, day; IVA, ivacaftor; LUM, lumacaftor; P, placebo; W, week 
Table 2. Absolute change from period baseline in ppFEV1 through week 8

\begin{tabular}{lll}
\hline & $\begin{array}{l}\text { PLACEBO } \\
\text { (N=18) }\end{array}$ & $\begin{array}{c}\text { LUM/IVA } \\
\text { (N=19) }\end{array}$ \\
\hline Mean period baseline ppFEV1 (SD), percentage points & $60.6(15.6)$ & \\
\hline Absolute change from period baseline through week 8 & & $3.2(1.1$ to 5.4$)$ \\
LS mean (95\% CI) & $1.1(-1.0$ to 3.3) & 0.005 \\
P value within treatment & 0.291 & \\
LS mean difference (95\% CI) & $2.1(-0.6$ to 4.8$)$ & 0.117 \\
\hline P value versus placebo & & \\
\hline
\end{tabular}

Figure 2. Absolute change in ppFEV,

a

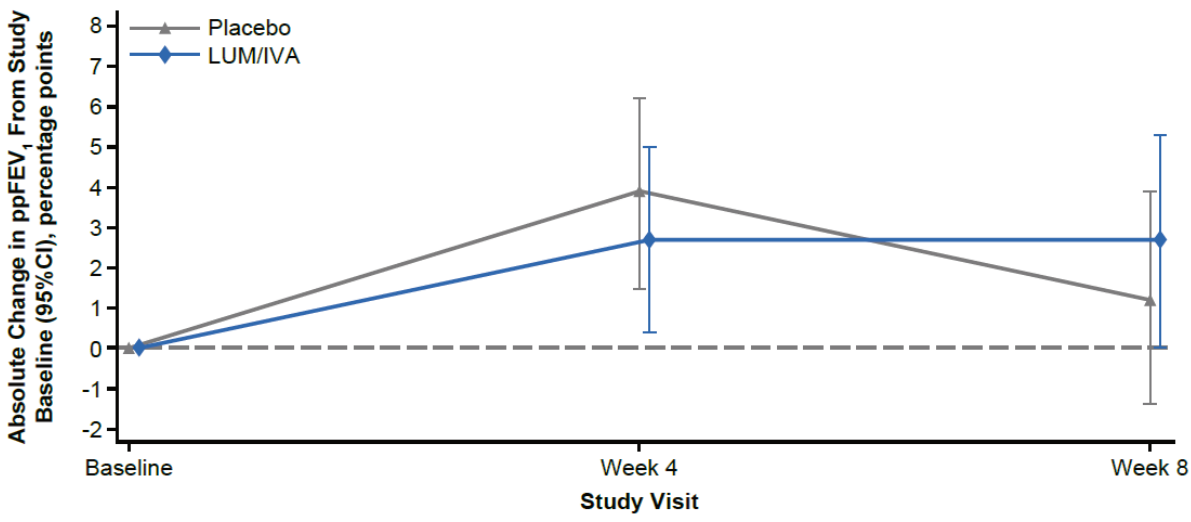

PLACEBO (N=18) LUM/IVA (N=19)

\begin{tabular}{llc}
\hline Mean study baseline ppFEV1 (SD), percentage points & $59.4(15.9)$ & $57.6(14.6)$ \\
$\begin{array}{l}\text { LS mean absolute change in ppFEV1 } \\
\text { trough week 8 (95\%CI), percentage points }\end{array}$ & $2.6(0.2-4.9)$ & $2.7(0.3-5.0)$ \\
p-value within treatment & 0.034 & 0.027 \\
LS mean difference (95\%CI), percentage points & & $0.1(-2.5$ to 2.7) \\
P-value vs placebo & 0.928 \\
\hline
\end{tabular}




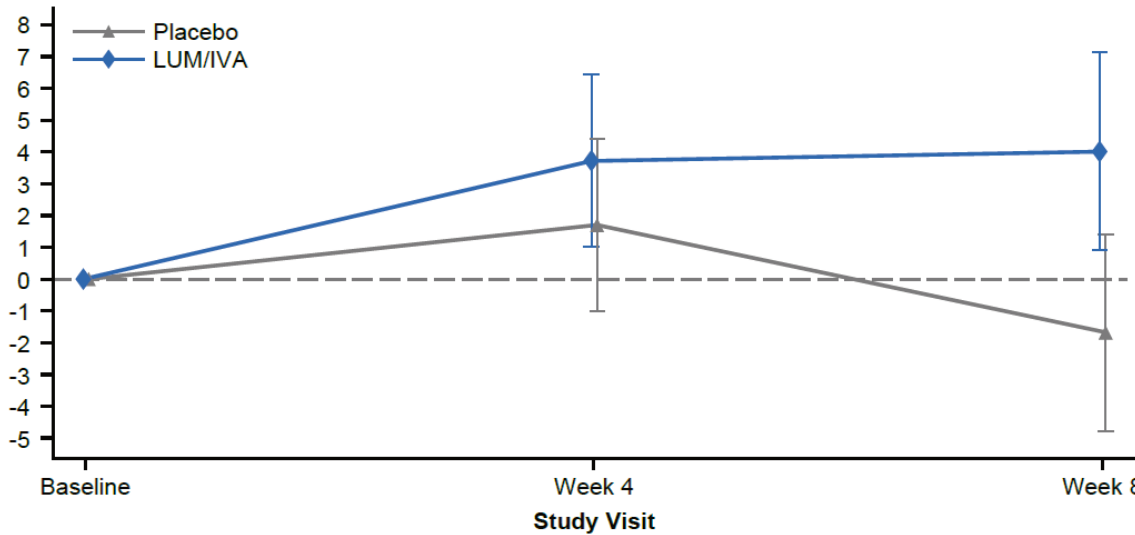

TREATMENT PERIOD 1

\begin{tabular}{llc}
\hline & PLACEBO (N=10) & LUM/IVA (N=10) \\
\hline Mean study baseline ppFEV1 (SD), percentage points & $60.0(20.0)$ & $57.7(9.4)$ \\
$\begin{array}{l}\text { LS mean absolute change in ppFEV1 } \\
\text { trough week 8 (95\%CI), percentage points }\end{array}$ & $0.0(-2.7-2.7)$ & $3.9(1.2-6.6)$ \\
P-value within treatment & 0.972 & $3.8(0.0-7.7)$ \\
LS mean difference (95\%Cl), percentage points & & 0.049 \\
\hline P-value vs placebo & & \\
\hline
\end{tabular}

(a) Change from study baseline through week 8 of treatment

(b) Change from period baseline through week 8 of treatment within treatment period 1. All patients received LUM $400 \mathrm{mg}$ /IVA $250 \mathrm{mg}$ every 12 hours (blue line) for 8 weeks and placebo (grey line) for 8 weeks according to 1 of 2 treatment sequences (LUM/IVA-placebo or placebo-LUM/IVA) with an 8-week washout period in between. Absolute change expressed as least squares (LS) mean (95\% CI).

CI, confidence interval; IVA, ivacaftor; LS, least squares; LUM, lumacaftor; ppFEV1, percent predicted forced expiratory volume in 1 second

The mean absolute change in sweat chloride from study baseline through week 8 showed a treatment difference of $-7.8 \mathrm{mmol} / \mathrm{L}(95 \% \mathrm{Cl},-12.6$ to $-3.1 ; \mathrm{P}=0.004)$ between the LUM/ IVA group and the placebo group (Fig. 3). A change of $-7.1(\mathrm{SE}, 1.7) \mathrm{mmol} / \mathrm{L}$ within the LUM/IVA group and a change of $0.7(S E, 1.8) \mathrm{mmol} / \mathrm{L}$ within the placebo group were observed.

The mean absolute change in CFQ-r respiratory domain score from study baseline to the end of week 8 showed a treatment difference of 3.5 points $(95 \% \mathrm{Cl},-6.4$ to $13.4 ; \mathrm{P}=0.469)$ between the LUM/IVA group and the placebo group. A change of 6.4 (SE, 3.9) points 
Figure 3. Absolute change from study baseline in sweat chloride concentration through week 8 of treatment

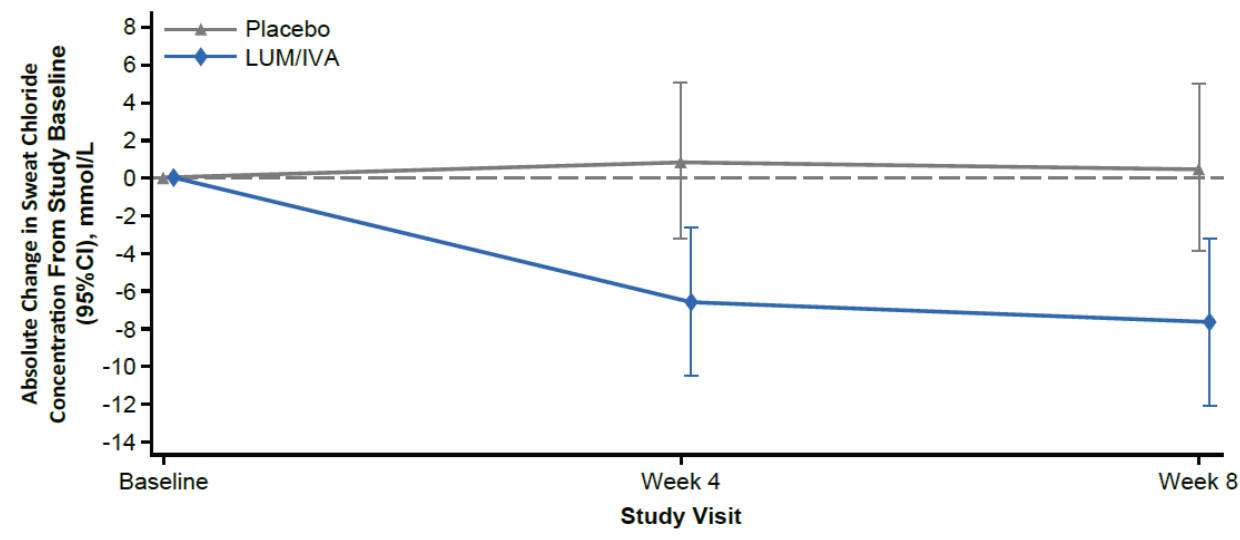

\begin{tabular}{|c|c|c|}
\hline & PLACEBO ( $\mathrm{N}=18)$ & LUM/IVA (N=19) \\
\hline $\begin{array}{l}\text { Mean baseline sweat chloride concentration (SD), } \\
\mathrm{mmol} / \mathrm{L}\end{array}$ & $81.6(9.1)$ & $80.1(10.5)$ \\
\hline $\begin{array}{l}\text { LS mean absolute change in sweat chloride } \\
\text { concentration trough week } 8(95 \% \mathrm{Cl}), \mathrm{mmol} / \mathrm{L}\end{array}$ & $0.7(-3.0-4.4)$ & $-7.1(-10.7--3.5)$ \\
\hline p-value within treatment & 0.688 & 0.0004 \\
\hline LS mean difference ( $95 \% \mathrm{CI})$, percentage points & & $-7.8(-12.6--3.1)$ \\
\hline p-value vs placebo & & 0.004 \\
\hline
\end{tabular}

All patients received LUM 400 mg/IVA 250 mg every 12 hours (blue line) or placebo (grey line) for 8 weeks according to 1 of 2 treatment sequences (LUM/IVA-placebo or placebo-LUM/IVA). Absolute change expressed as LS mean ( $95 \%$ CI).

CI, confidence interval; IVA, ivacaftor; LS, least squares; LUM, lumacaftor

within the LUM/IVA group and a change of $2.9(\mathrm{SE}, 4.0)$ points within the placebo group were observed.

\section{Safety}

Administration of LUM/IVA in this CF population for approximately 8 weeks was generally safe and well tolerated. Thirteen (72.2\%) patients receiving placebo and 15 (78.9\%) patients receiving LUM/IVA had at least $1 \mathrm{AE}$ in a given treatment period. Patients on placebo had AEs that were of mild $(55.6 \%)$ or moderate $(16.7 \%)$ severity. In patients who received LUM/ 
IVA, the majority of AEs were also considered mild (47.4\%) or moderate $(26.3 \%)$ in severity; $1(5.3 \%$ ) patient had a severe (grade 3 ) AE of hypertension, which resolved without change in LUM/IVA dosing and was considered unlikely to be related to LUM/IVA. No patients had serious AEs, or AEs that led to treatment discontinuation or interruption. No new unexpected AEs were identified. Overall, the safety results were consistent with the known safety profile of LUM/IVA established in previous Phase 3 studies..$^{15,24}$

\section{DISCUSSION}

Demonstrating clinical efficacy of novel therapies targeting rare mutations or small patient populations is challenging. This exploratory study that evaluated the efficacy of LUM/IVA in patients with CF aged $\geq 12$ years of age with at least one A455E-CFTR mutation encountered some of those challenges.

The primary endpoint, absolute change in ppFEV1 from study baseline through 8 weeks of treatment, did not show a significant treatment difference between the placebo and LUM/IVA groups. During this study, 2 patients had substantial increases in ppFEV1 after 8 weeks of LUM/IVA treatment in treatment period 1, but their ppFEV1 values did not return to study baseline level after the 8 -week washout period. Therefore, given that the sample size of this study was small, estimation of treatment effect based on the changes from study baseline can be impacted substantially by these 2 outlier patients, due to the underlying assumption of equal baselines for treatment period 1 and treatment period 2. The pre-specified supportive analysis of the changes in ppFEV1 from period baseline does not depend on such an assumption and showed a treatment difference of 2.1 percentage points between LUM/IVA and placebo. For future studies, ppFEV1 is known to be variable which could be a contributing factor to the results of this study, along with limited sample size. The use of an alternative primary endpoint, such as the changes from period baseline in ppFEV1 or sweat chloride concentration, might be helpful for the novel therapies targeting rare mutations or small patient populations. Studies of longer duration may also allow for the analysis of additional outcome measures.

While the study failed to meet the primary endpoint, it is important to note the treatment difference observed between LUM/IVA and placebo in sweat chloride concentration and in CFQ-r respiratory domain score. A trend in treatment difference between LUM/IVA and placebo was also observed with the treatment period 1 ppFEV1 analysis. Taken together, the overall efficacy results were suggestive of a clinical benefit with LUM/IVA.

Administration of LUM 400 mg/IVA $250 \mathrm{mg}$ q12h for up to 8 weeks was safe and well tolerated in patients with CF with the A455E-CFTR mutation. Safety results were consistent with those seen in other trials, and no new unexpected AEs were identified. 


\section{CONCLUSION}

Despite the study not meeting the primary endpoint of improvement in ppFEV ${ }_{1}$, LUM/IVA showed an improvement in sweat chloride concentration and CFQ-r respiratory domain score compared with placebo. Overall efficacy results from primary and other endpoints were suggestive of a potential clinical benefit with LUM/IVA in patients with CF and a A455E-CFTR mutation.

\section{ACKNOWLEDGEMENTS}

The authors thank the patients and their families, the trial coordinators, and the investigators for their contributions to the trials. Maggie Lee, PharmD, of Vertex Pharmaceuticals Incorporated, provided editorial coordination and assistance. JoAnna Anderson, PhD, and Katherine Mills-Lujan, PhD, CMPP, of ArticulateScience LLC provided editorial assistance under the direction of the authors, with support from Vertex Pharmaceuticals Incorporated. Support Statement: This study was supported by Vertex Pharmaceuticals Incorporated. 


\section{REFERENCES}

1. Kreindler JL. Cystic fibrosis: exploiting its genetic basis in the hunt for new therapies. Pharmacol Ther. 2010;125(2):219-229.

2. Cystic Fibrosis Foundation Patient Registry 2017 Annual Data Report.

3. Flume PA, Van Devanter DR. State of progress in treating cystic fibrosis respiratory disease. BMC Med. 2012;10:88.

4. Sheppard DN, Ostedgaard LS, Winter MC, Welsh MJ. Mechanism of dysfunction of two nucleotide binding domain mutations in cystic fibrosis transmembrane conductance regulator that are associated with pancreatic sufficiency. EMBO J. 1995;14(5):876-883.

5. Dekkers JF, Gogorza Gondra RA, Kruisselbrink E, et al. Optimal correction of distinct CFTR folding mutants in rectal cystic fibrosis organoids. Eur Respir J. 2016;48(2):451-458.

6. Cebotaru L, Rapino D, Cebotaru V, Guggino WB. Correcting the cystic fibrosis disease mutant, A455E CFTR. PLoS One. 2014;9(1):e85183.

7. Van Goor F, Yu H, Burton B, Hoffman BJ. Effect of ivacaftor on CFTR forms with missense mutations associated with defects in protein processing or function. J Cyst Fibros. 2014;13(1):29-36.

8. McKone EF, Emerson SS, Edwards KL, Aitken ML. Effect of genotype on phenotype and mortality in cystic fibrosis: a retrospective cohort study. Lancet (London, England). 2003;361(9370):1671-1676.

9. Nederlandse CF Registratie 2017.

10. Collée JM, de Vries HG, Scheffer H, Halley DJ, ten Kate LP. Relative frequencies of cystic fibrosis mutations in The Netherlands as an illustration of significant regional variation in a small country. Hum Genet. 1998;102(5):587-590.

11. Gan K-H, Veeze HJ, van den Ouweland A, et al. A Cystic Fibrosis Mutation Associated with Mild Lung Disease. N Engl J Med. 1995;333(2):95-99.

12. De Braekeleer M, Allard C, Leblanc JP, Simard F, Aubin G. Genotype-phenotype correlation in cystic fibrosis patients compound heterozygous for the A455E mutation. Hum Genet. 1997;101(2):208-211.

13. Lilley M, Christian S, Hume S, et al. Newborn screening for cystic fibrosis in Alberta: Two years of experience. Paediatr Child Health. 2010;15(9):590-594.

14. Sawicki G, Konstan M, McKone $\mathrm{E}$, et al. Rate of lung function decline in patients with cystic fibrosis (cf) having a residual function gene mutation. In: Clinical Implications of Cystic Fibrosis. BMJ Publishing Group Ltd and British Thoracic Society; 2017:A222.1-A222.

15. Wainwright CE, Elborn JS, Ramsey BW, et al. Lumacaftor-Ivacaftor in patients with cystic fibrosis homozygous for Phe508del CFTR. N Engl J Med. May 2015.

16. Boston MA Vertex Pharmaceuticals Incorporated. Orkambi (lumacaftor/ivacaftor) [package insert]. 2018.

17. London UK: Vertex Pharmaceuticals (Europe) Limited. Orkambi (lumacaftor/ivacaftor) [summary of product characteristics]. 2018.

18. Dekkers JF, Wiegerinck $\mathrm{CL}$, de Jonge $\mathrm{HR}$, et al. A functional CFTR assay using primary cystic fibrosis intestinal organoids. Nat Med. 2013;19(7):939-945.

19. Dekkers JF, Berkers G, Kruisselbrink E, et al. Characterizing responses to CFTR-modulating drugs using rectal organoids derived from subjects with cystic fibrosis. Sci Transl Med. 2016;8(344).

20. London UK: Vertex Pharmaceuticals (Europe) Limited. Kalydeco (ivacaftor) [summary of product characteristics]. 2018.

21. Day S, Jonker AH, Lau LPL, et al. Recommendations for the design of small population clinical trials. Orphanet J Rare Dis. 2018;13(1):195. 
22. Lillie EO, Patay B, Diamant J, Issell B, Topol EJ, Schork NJ. The n-of-1 clinical trial: the ultimate strategy for individualizing medicine? Per Med. 2011;8(2):161-173.

23. Quanjer $\mathrm{PH}$, Stanojevic S, Cole TJ, et al. Multi-ethnic reference values for spirometry for the 3-95-yr age range: the global lung function 2012 equations. Eur Respir J. 2012;40(6):13241343.

24. Ratjen F, Hug C, Marigowda G, et al. Efficacy and safety of lumacaftor and ivacaftor in patients aged 6?11 years with cystic fibrosis homozygous for F508del-CFTR : a randomised, placebo-controlled phase 3 trial. Lancet Respir Med. June 2017. 


\section{CHAPTER \\ 6}

FORSKOLIN-INDUCED SWELLING OF INTESTINAL ORGANOIDS CORRELATES WITH DISEASE SEVERITY IN ADULTS WITH CYSTIC FIBROSIS AND HOMOZYGOUS F508DEL MUTATIONS

Karin M. de Winter - de Groot, Gitte Berkers, Rozemarijn E.P. Marck - van der Wilt, Renske van der Meer, Annelotte Vonk, Johanna F. Dekkers, Margot Geerdink, Sabine Michel, Evelien Kruisselbrink, Robert G.J. Vries, Hans Clevers, Frank P. Vleggaar, Sjoerd G. Elias, Harry G.M. Heijerman, Cornelis K. van der Ent, Jeffrey M. Beekman 


\section{ABSTRACT}

Objective: Cystic fibrosis transmembrane conductance regulator (CFTR) function measurements in intestinal organoids may help to better characterize individual disease expression in F508del homozygous people. Our objective was to study correlations between CFTR function as measured with forskolin-induced swelling (FIS) in rectal organoids with clinical parameters in adult cystic fibrosis (CF) patients with homozygous F508del mutations.

Methods: Multicenter observational study. Thirty-four adults underwent rectal biopsy, pulmonary function tests (forced expiratory volume in 1 second and forced vital capacity, FEV1 and FVC respectively), chest X-ray and chest computed tomography (CT). Body mass index (BMI) was assessed at study visit and exacerbation rate was determined during five years prior to study visit. Organoids were cultured and measured after stimulation with $5 \mu \mathrm{m}$ forskolin for three hours to quantitate CFTR residual function.

Results: FIS was positively correlated with FEV1 ( $r=0.36,95 \% \mathrm{Cl} 0.02-0.62, \mathrm{p}=0.04$ ) and $\mathrm{BMI}(r=0.42,95 \% \mathrm{Cl} 0.09-0.66, \mathrm{p}=0.015)$. FIS was negatively correlated with PRAGMA-CF CT score for $\%$ of disease $(r=-0.37,95 \% \mathrm{Cl}-0.62--0.03, p=0.049)$. We found no significant correlation between FIS and chest radiography score for CF $(r=-0.16,95 \% \mathrm{Cl}-0.48-0.20, p=$ $0.44)$. We observed a trend between higher FIS and a lower mean number of exacerbations over the last 5 years of observation, but this was not statistically significant (Poisson regression, $\mathrm{p}=0.089$ ).

Conclusion: FIS of intestinal organoids varied between subjects with homozygous F508del and correlated with pulmonary and nutritional parameters. These findings suggest that differences at low CFTR residual function may contribute to clinical heterogeneity in F508del homozygous patients and small changes in CFTR residual function might impact long-term disease expression. 


\section{INTRODUCTION}

Patient-derived cell cultures such as organoids can function as living biomarkers to help to understand and predict individual disease progression. Organoids are multicellular, three-dimensional epithelial structures that self-organize in vitro and recapitulate the in vivo epithelial tissue including stem cell function, polarity and spatial organization of cell subsets.1 Rectal organoids have been used to study cystic fibrosis (CF), a monogenetic disease caused by mutations in the Cystic Fibrosis Transmembrane Conductance Regulator (CFTR) gene. ${ }^{2-5}$ CF is a chronic, progressive disease and associated with significant variability in disease progression. Genetic variation in CFTR, disease modifier genes and environmental factors drive the individual phenotype, and contribute to varying degrees of distinct organ-related clinical phenotypes (www.cftr2.org)..$^{6-12}$

Previous studies demonstrate that particular CFTR mutations are associated with high residual function, and link to milder disease phenotypes (www.cftr2.org). The contribution of residual function to variation of disease in people with identical mutations remains much less understood. This is also true for the approximately $50 \%$ of people with CF who are homozygous for F508del mutation (F508del-HZ) and show clear differences in disease progression.7,13,14 Several studies suggest that differences in F508del protein localization and function (as measured with sweat chloride concentration (SCC), intestinal current measurement (ICM) or nasal potential difference (NPD)) exist. ${ }^{13,15-19}$ In contrast, other studies suggest lack or very low function of F508del. ${ }^{20}$ This illustrates that additional biomarkers of CFTR may help to better understand to what extent variety in CFTR function contributes to individual disease progression in people having two F508del CFTR mutations. ${ }^{12,13,21,22}$

CFTR function can be visualized in rectal organoids upon stimulation with a cAMP-raising drug such as forskolin, which leads to CFTR channel opening and transport of ions and fluids into the organoid lumen causing rapid organoid swelling. This forskolin-induced swelling (FIS) assay appears fully dependent on CFTR in rectal organoids, and can be used to precisely quantitate CFTR function using forskolin dose ranges. ${ }^{3}$ We also observed that individual FIS is stable upon prolonged culture and after biobanking. ${ }^{3}$ Large differences in FIS stratify for disease severity in young children with a range of CFTR mutations at 1 years of age. ${ }^{23} \mathrm{FIS}$ of F508del-HZ organoids is clearly lower from FIS of organoids from children with milder disease, and was within a range of none-to-minimal residual function., 3,23 Interestingly, careful analysis of our previously published data showed that the majority of the F508del-HZ organoids displayed at least some forskolin-induced luminal fluid secretion supporting limited residual CFTR function. ${ }^{24}$ However, such limited fluid secretory responses were difficult to precisely quantitate with FIS at 60 min due to the signal-tonoise ratio in the previous study. We here used prolonged forskolin stimulation (3 hours instead of $60 \mathrm{~min}$ ) of F508del-HZ organoids to more precisely quantify low amounts of CFTR residual function, and studied correlations of FIS with relevant clinical outcome parameters in 34 adults with homozygous F508del mutations. 


\section{METHODS}

\section{Study design and participants}

A multicentre observational study was performed in two CF centres (Haga Teaching Hospital, The Hague and University Medical Centre Utrecht, Utrecht) in The Netherlands. Between Dec 2013 and Apr 2015, 34 adults with F508del homozygosity and a clinically stable condition enrolled in this study. The diagnosis of CF was confirmed according to regular practice by clinical signs of disease and/or increased sweat chloride concentrations ( $\geq 60 \mathrm{mmol} / \mathrm{L}$ ) and presence of $2 \mathrm{dF508}$ CFTR mutations.

The study was approved by the Ethics Committee of the University Medical Centre Utrecht, The Netherlands. Written informed consent was obtained from all participants.

\section{Clinical measurements}

During a study visit all subjects underwent a rectal biopsy as well as pulmonary function tests (forced expiratory volume in 1 second and forced vital capacity, FEV and FVC respectively), chest X-ray and chest computed tomography (CT). Relevant clinical parameters on nutritional status (body mass index, BMI) were measured during the study visit and exacerbation rate was determined during the five years prior to the study visit. We used the definition as described by Tregiarri et al. ${ }^{25}$ to establish the presence of a pulmonary exacerbation. In the digital information systems all antibiotic courses in the past 5 years of each individual patient were counted, and it was checked whether antibiotic courses were prescribed for a pulmonary exacerbation and if the Treggiari criteria were met. Pancreatic insufficiency (PI) was defined by a history of malabsorption and fecal elastase (FE) levels $<15 \mu \mathrm{g} / \mathrm{g}$.

Pulmonary function tests, chest X-ray and CT were performed according to standardized procedures. ${ }^{26,27}$ Values of FVC and $\mathrm{FEV}_{1}$, were expressed as percentage of predicted normal values for sex and height (ppFVC and ppFEV ${ }_{1}$, respectively), and each individual's highest values of the last year were used for analyses. Chest X-rays were scored using a Modified Crispin-Norman chest radiography score for CF. ${ }^{28}$ See online supplements (Supplemental Table 51) for CT procedures. Severity of airway disease (\% disease) was scored in random order, blinded to patient identifiers using the Perth-Rotterdam Annotated Grid Morphometric Analysis for CF CT scoring method (PRAGMA-CF CT score). ${ }^{26}$ Disease percentage reflects the fraction of total lung volume showing bronchiectasis, bronchial thickening, or mucus plugging. All subjects did not receive CFTR modulators before or during the study.

\section{Organoid measurements}

Methods for generation of organoids have been described previously. $2,3,5$ We adapted 
the protocol by stimulating the F508del-HZ organoids with $5 \mu \mathrm{m}$ forskolin for three hours to detect variation in CFTR residual function. The total organoid area (xy plane in $\mu \mathrm{m}^{2}$ ) increase relative to $t=0$ of forskolin treatment was quantified using Zen Image analysis software module (Zeiss). Area under the curve (AUC) ( $t=180 \mathrm{~min}$; baseline, $100 \%$ ) was calculated using Excel. Measurements were done in quadruple (minimal $n=2$ experiments) in batches of 12 organoid cultures. Analysts who executed the FIS assays were blinded to patient characteristics.

\section{Statistical analysis}

Pearson's correlation was calculated between the in vitro CFTR function, as measured in the intestinal organoid model, and in vivo clinical parameters as percentage predicted forced expiratory volume in 1 second ( $p$ FEEV ${ }_{1}$ ), BMI, PRAGMA-CF CT score (\% disease) and Modified Crispin-Norman chest radiography score for CF, which were all approximate normally distributed data. We used linear regression to adjust the association between FIS and these clinical parameters for age. Similarly, we used Poisson regression to assess the association between pulmonary exacerbations of the last 5 years and FIS values. The best Poisson-derived fit and 95\% confidence interval (CI) was calculated. Statistical analysis were performed using GraphPad Prism 7.02 and R 3.2.1 for Mac and p-values were reported based on two-sided tests. $P<0.05$ indicates statistical significance.

\section{Role of the funding source}

The study sponsor did not have a role in the study design; in the collection, analysis, and interpretation of data; in the writing of the report; and in the decision to submit the paper for publication. The corresponding author had full access to all data in the study and had final responsibility for the decision to submit for publication.

\section{RESULTS}

Table 1 shows the clinical characteristics of all 34 subjects. The youngest patient was 18 years old and the oldest 51 years. BMl varied between 14.2 and $23.8 \mathrm{~kg} / \mathrm{m}^{2}$ and all patients had pancreatic insufficiency. Lowest SCC was $62 \mathrm{mmol} / \mathrm{L}$ and highest 150 $\mathrm{mmol} / \mathrm{L}$. Pulmonary disease also differed between patients with lowest $\mathrm{ppFEV}$, and ppFVC values of 27 and 31, respectively and highest values of 98 and 112, respectively.

FIS values of organoids of all subjects are shown in Figure 1. All organoids showed some FIS response which increased linearly during the 3 hours of stimulation with forskolin (supplemental Fig. S1), indicating limited overall swelling. FIS (area under the curve (AUC) of 3 h swell curves) was positively correlated with ppFEV $(r=0.36,95 \% \mathrm{Cl} 0.02-0.62, p=$ 
Table 1. Patient characteristics at inclusion of all subjects, $n=34$

\begin{tabular}{|c|c|}
\hline \multicolumn{2}{|l|}{$\operatorname{Sex}(n[\%])$} \\
\hline Female & $12[35]$ \\
\hline Male & $22[65]$ \\
\hline Age (years; median [IQR]) & $27.5[23-33.8]$ \\
\hline Age at diagnosis (years; median [IQR]) & $0[0-1]$ \\
\hline \multicolumn{2}{|l|}{ Location of CF care (n [\%]) } \\
\hline Utrecht & $25[74]$ \\
\hline Haga & $9[26]$ \\
\hline BMI (kg/m2; mean [SD]) & $19.0[2.4]$ \\
\hline Sweat chloride concentration (mmol/L; mean [SD]\# & $106.0[19.9]$ \\
\hline \multicolumn{2}{|l|}{ Pancreatic sufficiency (n [\%]) } \\
\hline Sufficient (FE > 200 g/g) & $\mathrm{O}[0]$ \\
\hline Insufficient (FE < $15 \mu \mathrm{g} / \mathrm{g}$ ) & 34 [100] \\
\hline Pseudomonas colonization (n [\%]) & $19[56]$ \\
\hline Antibiotic treatmentsฐ (median [IQR]) & $7[3-11]$ \\
\hline $\begin{array}{l}\text { Maintenance antibiotic treatment, oral and / or by } \\
\text { inhalation }(\mathrm{n}[\%])\end{array}$ & $34[100]$ \\
\hline CFRD (n [\%]) & $5[14.7]$ \\
\hline \multicolumn{2}{|l|}{ Lung Function* (median [IQR]) } \\
\hline ppFEV1 & $59.8[45.8-84.7]$ \\
\hline ppFVC & $73.7[59.9-85.0]$ \\
\hline Crispin Norman (mean [SD]) & $20.7[5.1]$ \\
\hline \multicolumn{2}{|l|}{ PRAGMA-CF CT scores (mean [SD]) } \\
\hline \% bronchiectasis & $12.2[4.5]$ \\
\hline$\%$ air trapping & $52.5[18.4]$ \\
\hline$\%$ disease & $26.5[8.1]$ \\
\hline
\end{tabular}

\# known of $n=17$ subjects. § Number of antibiotic treatments (oral or iv) in the last 5 years because of pulmonary exacerbations. *Expressed as percentage predicted (pp).

BMI, body mass index; CFRD, CF-related diabetes; FE, fecal elastase; IQR, inter quartile range; ppFEV1, percentage predicted forced expiratory volume in 1 second; PpFVC, percentage predicted forced vital capacity; PRAGMA-CF, PerthRotterdam annotated grid morphometric analysis for CF; SD, standard deviation. 
Figure 1. Results of FIS of organoids of all 34 subjects at $5 \mu \mathrm{M}$ forskolin after 180 min

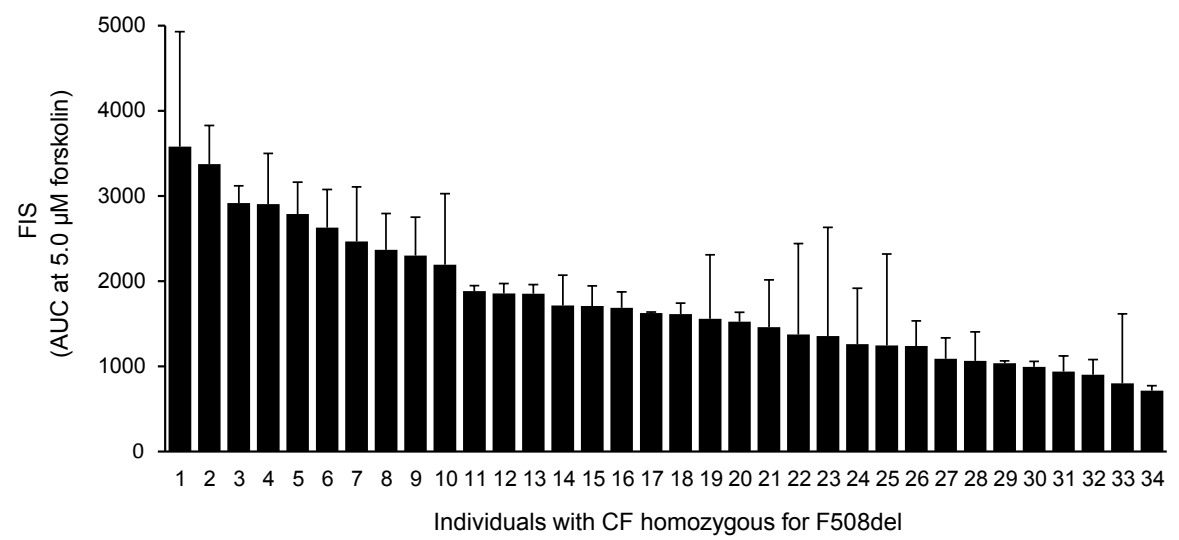

Each bar represents AUC values averaged from three independent experiments per individual (mean \pm standard deviation (SD)).

0.04, Fig. 2a) and BMI ( $r=0.42,95 \% \mathrm{Cl} 0.09-0.66, \mathrm{p}=0.015$, Fig. 2b). FIS was negatively correlated with PRAGMA-CF CT score for $\%$ of disease $(r=-0.37,95 \% \mathrm{Cl}-0.62--0.03, p=$ 0.049 , Fig. 2c). We found no significant correlation between FIS values and the Modified Crispin-Norman chest radiography score for CF ( $r=-0.16,95 \% \mathrm{Cl}-0.48-0.20, p=0.44$, Fig. $2 d$ ). With increasing FIS values, we found a lower mean number of exacerbations over the last 5 years of observation, but this was not significant (Poisson regression, $p=0.089$ ), see Figure 3. Linear regression analysis adjusted for age also showed a significant association between FIS values and ppFEV 1 , PRAGMA-CF CT score and BMI. $\beta$ values changed less than $10 \%$ when age was added to the regression model, the results of the unadjusted and adjusted linear regression model are described in an online supplemental Table S2.

\section{DISCUSSION}

This study investigated the relationship between CFTR residual function and clinical parameters in F508del-HZ adults. We found that FIS of intestinal organoids varied between subjects with identical mutations (Fig. 1), showing that limited residual CFTR function is present and variable in cultured intestinal tissue in this group of subjects. Previous data suggests that FIS in rectal organoids is fully CFTR-dependent, indicating 
Figure 2. Relationship between FIS and clinical outcomes in intestinal organoids of subjects with CF homozygous for F508del

a.

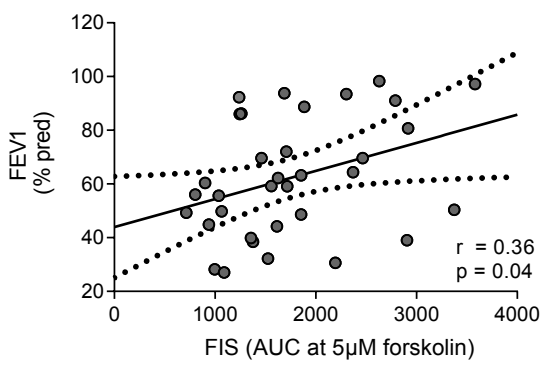

C.

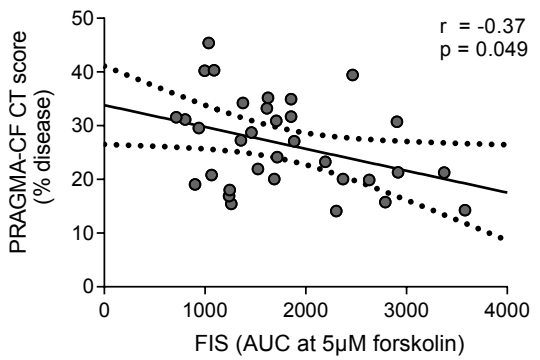

b.

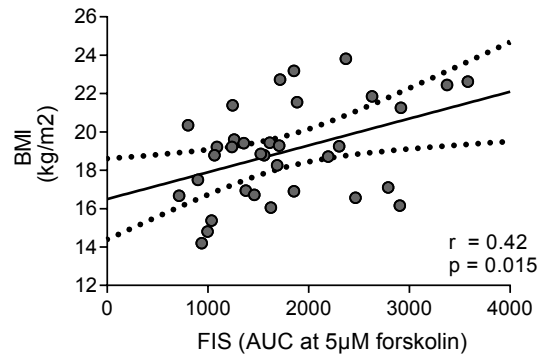

d.

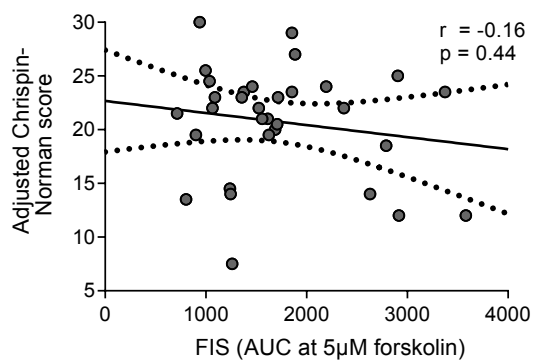

Pearson correlation between FIS and

(a) pulmonary function (ppFEV1, 95\% Cl 0.02-0.62, $\mathrm{p}=0.04$ ),

(b) nutritional status (BMI, 95\% Cl 0.09-0.66, $\mathrm{p}=0.015$ and after adjustment for age $\mathrm{p}=0.015$ ), and pulmonary structural abnormalities shown on

(c) chest CT (PRAGMA-CF CT score, 95\% Cl -0.62- -0.03, $\mathrm{p}=0.036$ and after adjustment for age $\mathrm{p}=0.049$ ) and

(d) chest X-ray (adjusted Crispin-Norman score, 95\% Cl -0.48-0.20, p=0.37 and after adjustment for age $\mathrm{p}=0.44$ ).

that the differences in FIS are most likely due to variation in CFTR protein (apical amount, open-probability or conductance) or other non-CFTR related biological pathways that impact the electrochemical driving force for CFTR-dependent ion transport. 29,30

CF subjects with higher FIS values showed a significantly better BMI, higher FEV 1 and a lower chest CT score than subjects with lower FIS (Fig. 2). Furthermore, CF subjects with lower FIS values of intestinal organoids showed a trend to having more pulmonary exacerbations in the past 5 years. Another study from our group, which was recently published, showed that FIS defined CF subgroups with distinct clinical parameters such as pancreatic sufficiency, nutritional status, PRAGMA-CF CT score and infant lung 
Figure 3. Relationship between FIS and number of pulmonary exacerbations in the past 5 years, adjusted for age (Poisson regression analysis, $\mathrm{p}=0.089$ )

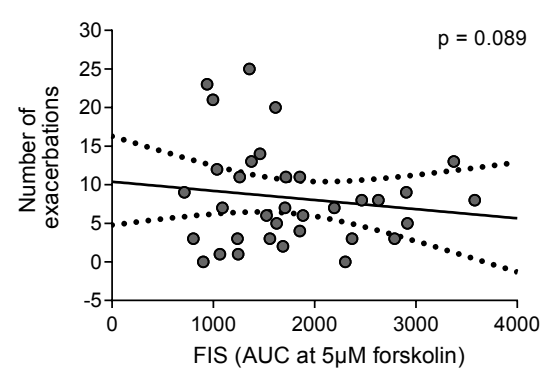

Dashed lines indicate $95 \% \mathrm{Cl}$.

function in a group of 34 infants of one year of age and 17 different CFTR mutations. ${ }^{23}$ The correlation between FIS in intestinal organoids and relevant clinical parameters in this study and previously published data ${ }^{2,3}$ support that the FIS assay as biomarker of CFTR function captures disease characteristics in vitro over a wide range of CFTRdependent functions.

In contrast to our previous study that focused on a diverse set of CFTR genotypes, this study suggests that differences in clinical characteristics in adults with the same genotype might relate to small differences in CFTR function. This suggests that very low quantities of CFTR function might have a measurable impact on disease progression, although long-term studies are required to confirm this.

Consistent with our previous report ${ }^{23}$, it supports the initiation of new studies focusing on the additional classification of patients based on different amounts of CFTR residual function using FIS in organoids.

The distribution of variation of FIS between F508del homozygous patients differs to studies which have used other biomarkers of CFTR function. ${ }^{11,12,17,31}$ In organoids in the current study, we did not observe residual CFTR function levels that were associated with residual function in patients with milder CFTR mutations and pancreatic sufficiency. This is different to some SCC and ICM studies, as some patients with severe CFTR mutations and pancreatic insufficiency can have ICM or SCC levels similar to those observed in patients with milder mutations. ${ }^{23,31}$ At the group level some studies were able to detect phenotypically distinct characteristics in respect to pulmonary function and/or nutritional status in F508del cystic fibrosis twins and siblings. ${ }^{11,12}$ However, they 
did not or were not able to show a correlation of this variability to disease severity on the individual level. One study used NPD to measure CFTR function in 27 men who were homozygous for F508del mutations. ${ }^{13}$ They found a similar positive correlation between responses to isoprenaline and $\mathrm{FEV}_{1}$, also suggesting that more residual function appears to lead to less severe lung disease. They did not show this relationship with other CFTR biomarkers or for females or other clinical parameters. Our study is in overall agreement with these previous reports that also support that variation in CFTR function can be a clinically relevant factor for disease expression in F508del homozygote patients.

The data suggest that small differences in CFTR function may impact individual CF phenotype. This may be important for understanding how much CFTR modulation is required in the context of pharmacological repair of CFTR by CFTR modulators. Efficacy of these modulators are mostly studied in short-term clinical trials and changes in ppFEV, or sweat chloride upon short-term treatment supported efficacy of e.g. lumacaftor and ivacaftor in people homozygous for F508del, but not in people with a single F508del allele $^{32}$. The increase in CFTR function upon in vitro CFTR modulation by lumacaftor/ ivacaftor in F508del single allele organoids ${ }^{2,3}$ is larger as compared to the differences in residual function observed here between patients. This might indicate that longer studies might have demonstrated treatment effects of lumacaftor/ivacaftor in F508del single allele patients as well. More precise typing of the relationship between in vitro organoid function and long term clinical disease may help to select long term beneficiaries of CFTR modulators, which could be especially relevant for people expressing rare variants of CFTR for who establishing individual efficacy of treatment may be important for access and reimbursement.

There are several limitations in this study. First, the correlations with FIS and nutritional and pulmonary parameters were significant, but not very strong. This lowers the positive and negative predictive values of the FIS for a particular disease phenotype. This is consistent with findings of genetic studies and the contribution of genes and environment to individual disease characteristics such as airway obstruction and lower $\mathrm{BM} \mathrm{M}^{9,33,34}$. Other limitations of the study include a relatively small, cross-sectional patient cohort. Furthermore, there is no agreed definition of a pulmonary exacerbation and length of antibiotic courses may have varied in and between patients. This might have affected the strength and significance of the correlation. Additionally, organoid swelling is not a direct readout of CFTR function, it relies on coupling of CFTR-dependent ion transport to fluid transport. The organoid measurements also require considerable local expertise and expensive equipment. Finally, although patients were all F508del homozygous, additional variation in the CFTR alleles were not characterized, indicating that patients might have different F508del haplotypes. ${ }^{22,35}$ These limitations indicate a need for larger follow up studies. The validity of the organoid biomarker for prediction of long term outcomes needs to be confirmed in other populations, with the same, different or unknown CFTR genotypes. 


\section{CONCLUSION}

In conclusion, these findings suggest that variability of CFTR residual function could contribute to the significant heterogeneity in the F508del homozygous CF patient population. The findings in this study support that FIS in intestinal organoids can capture clinical disease features, even in individuals with identical CFTR mutations.

\section{ACKNOWLEDGEMENTS}

Funding.The HIT-CF program of the Dutch cystic fibrosis foundation (NCFS) and ZonMW (Dutch organization for health research and $\mathrm{D}=$ development) funded the study. 


\section{REFERENCES}

1. Sato T, Clevers H. Growing self-organizing mini-guts from a single intestinal stem cell: mechanism and applications. Science (80- ). 2013;340(6137):1190-1194.

2. Dekkers JF, Wiegerinck $\mathrm{CL}$, de Jonge $\mathrm{HR}$, et al. A functional CFTR assay using primary cystic fibrosis intestinal organoids. Nat Med. 2013;19(7):939-945.

3. Dekkers JF, Berkers G, Kruisselbrink E, et al. Characterizing responses to CFTR-modulating drugs using rectal organoids derived from subjects with cystic fibrosis. Sci Transl Med. 2016;8(344):344ra84.

4. Ratjen F, Döring G. Cystic fibrosis. Lancet. 2003;361(9358):681-689.

5. Boj SF, Vonk AM, Statia M, et al. Forskolin-induced Swelling in Intestinal Organoids: An \&lt;em\&gt;In vitro\&lt;/em\&gt; Assay for Assessing Drug Response in Cystic Fibrosis Patients. J Vis Exp. 2017;(120).

6. Kerem $\mathrm{E}$, Corey $\mathrm{M}$, Kerem $\mathrm{BS}$, et al. The relation between genotype and phenotype in cystic fibrosis--analysis of the most common mutation (delta F508). N Engl J Med. 1990;323(22):1517-1522.

7. Sosnay PR, Siklosi KR, Van Goor F, et al. Defining the disease liability of variants in the cystic fibrosis transmembrane conductance regulator gene. Nat Genet. 2013;45(10):11601167.

8. McKone EF, Emerson SS, Edwards KL, Aitken ML. Effect of genotype on phenotype and mortality in cystic fibrosis: a retrospective cohort study. Lancet. 2003;361(9370):16711676.

9. Cutting GR. Cystic fibrosis genetics: from molecular understanding to clinical application. Nat Rev Genet. 2014;(November).

10. Consortium TCFG-P. Correlation between Genotype and Phenotype in Patients with Cystic Fibrosis. N Engl J Med. 1993;329(18):1308-1313.

11. Mekus F, Ballmann M, Bronsveld I, Bijman J, Veeze H, Tümmler B. Categories of $\Delta F 508$ homozygous cystic fibrosis twin and sibling pairs with distinct phenotypic characteristics. Twin Res. 2000;3(04):277-293.

12. Bronsveld I, Mekus F, Bijman J, et al. Chloride conductance and genetic background modulate the cystic fibrosis phenotype of Delta F508 homozygous twins and siblings. J Clin Invest. 2001;108(11):1705-1715.

13. Thomas SR, Jaffe A, Geddes DM, Hodson ME, Alton EW. Pulmonary disease severity in men with $\triangle F 508$ cystic fibrosis and residual chloride secretion. Lancet. 1999;353(9157):984-985.

14. Kerem E, Corey M, Kerem B, et al. The Relation between Genotype and Phenotype in Cystic Fibrosis - Analysis of the Most Common Mutation ( $\triangle \mathrm{F} 508$ ). N Engl J Med. 1990;323(22):1517-1522.

15. van Meegen MA, Terheggen-Lagro SWJ, Koymans KJ, van der Ent CK, Beekman JM. Apical CFTR Expression in Human Nasal Epithelium Correlates with Lung Disease in Cystic Fibrosis. PLoS One. 2013;8(3)

16. Ho LP, Samways JM, Porteous DJ, et al. Correlation between nasal potential difference measurements, genotype and clinical condition in patients with cystic fibrosis. Eur Respir J. 1997;10(9):2018-2022.

17. Bronsveld I, Mekus F, Bijman J, et al. Residual chloride secretion in intestinal tissue of deltaF508 homozygous twins and siblings with cystic fibrosis. The European CF Twin and Sibling Study Consortium. Gastroenterology. 2000;119(1):32-40.

18. van Barneveld A, Stanke F, Tamm S, et al. Functional analysis of F508del CFTR in native human colon. Biochim Biophys Acta - Mol Basis Dis. 2010;1802(11):1062-1069.

19. Kälin N, Claaß A, Sommer M, Puchelle E, Tümmler B. $\triangle F 508$ CFTR protein expression in tissues from patients with cystic fibrosis. J Clin Invest. 1999;103(10):1379-1389. 
20. Mall M, Kreda SM, Mengos $\mathrm{A}$, et al. The $\triangle \mathrm{F} 508$ Mutation Results in Loss of CFTR Function and Mature Protein in Native Human Colon. Gastroenterology. 2004;126(1 SUPPL. 1):3241.

21. Hirtz S, Gonska T, Seydewitz $\mathrm{HH}$, et al. CFTR Cl- channel function in native human colon correlates with the genotype and phenotype in cystic fibrosis. Gastroenterology. 2004;127(4):1085-1095.

22. Vecchio-Pagán B, Blackman SM, Lee M, et al. Deep resequencing of CFTR in 762 F508del homozygotes reveals clusters of non-coding variants associated with cystic fibrosis disease traits. Hum genome Var. 2016;3:16038.

23. de Winter-de Groot KM, Janssens HM, van Uum RT, et al. Stratifying infants with cystic fibrosis for disease severity using intestinal organoid swelling as a biomarker of CFTR function. Eur Respir J. 2018;52(3):1702529.

24. Zomer-van Ommen DD, Vijftigschild LAW, Kruisselbrink E, et al. Limited premature termination codon suppression by read-through agents in cystic fibrosis intestinal organoids. J Cyst Fibros. 2016;15(2):158-162.

25. Treggiari MM, Rosenfeld M, Mayer-Hamblett N, et al. Early anti-pseudomonal acquisition in young patients with cystic fibrosis: Rationale and design of the EPIC clinical trial and observational study,. Contemp Clin Trials. 2009;30(3):256-268.

26. Rosenow T, Oudraad MCJ, Murray CP, et al. PRAGMA-CF. A Quantitative Structural Lung Disease Computed Tomography Outcome in Young Children with Cystic Fibrosis. Am J Respir Crit Care Med. 2015;191(10):1158-1165.

27. Quanjer PH, Stanojevic S, Cole TJ, et al. Multi-ethnic reference values for spirometry for the 3-95-yr age range: the global lung function 2012 equations. Eur Respir J. 2012;40(6):13241343.

28. de Jong PA, Achterberg JA, Kessels OAM, et al. Modified Chrispin-Norman chest radiography score for cystic fibrosis: observer agreement and correlation with lung function. Eur Radiol. 2011;21(4):722-729.

29. Foulke-Abel J, In J, Yin J, et al. Human Enteroids as a Model of Upper Small Intestinal Ion Transport Physiology and Pathophysiology. Gastroenterology. 2016;150(3):638-649.e8.

30. Beekman JM. Individualized medicine using intestinal responses to CFTR potentiators and correctors. Pediatr Pulmonol. 2016;51(S44):S23-S34.

31. Collaco JM, Blackman SM, Raraigh KS, et al. Sources of Variation in Sweat Chloride Measurements in Cystic Fibrosis. Am J Respir Crit Care Med. June 2016.

32. Boyle MP, Bell SC, Konstan MW, et al. A CFTR corrector (lumacaftor) and a CFTR potentiator (ivacaftor) for treatment of patients with cystic fibrosis who have a phe508del CFTR mutation: a phase 2 randomised controlled trial. Lancet Respir Med. 2014;2(7):527-538.

33. Vanscoy LL, Blackman SM, Collaco JM, et al. Heritability of Lung Disease Severity in Cystic Fibrosis. Am J Respir Crit Care Med. 2007;175(10):1036-1043.

34. Bradley GM, Blackman SM, Watson CP, Doshi VK, Cutting GR. Genetic modifiers of nutritional status in cystic fibrosis. Am J Clin Nutr. 2012;96(6):1299-1308.

35. Stanke F, Becker T, Kumar V, et al. Genes that determine immunology and inflammation modify the basic defect of impaired ion conductance in cystic fibrosis epithelia. J Med Genet. 2011;48(1):24-31. 


\section{SUPPLEMENTALS}

Supplemental Figure S1. Increase of FIS responses of all organoids during 3 hours of stimulation with forskolin (average per patient in quadruplo)

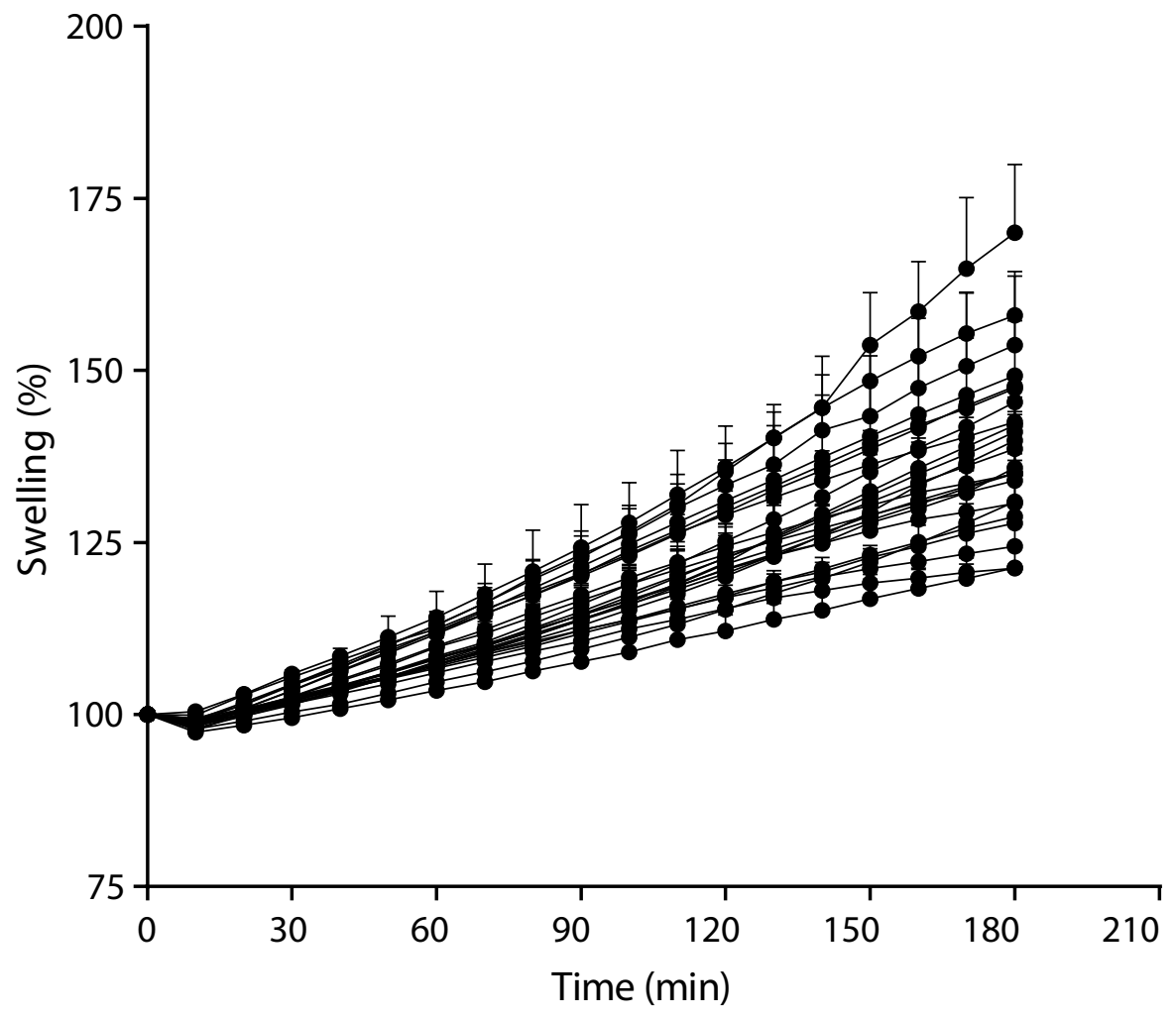


Supplemental Table S1. CT settings. CTDIvol and Dose-Length Product based on a 32-cm phantom and reported as mean

\begin{tabular}{ll}
\hline CT SETrING & UTRECHT \\
\hline CT Scanner & Philips Brilliance 16p \\
\hline Scan type & Inspiratory \\
\hline Acquisition & Helical \\
\hline Rotation time (ms) & 500 \\
\hline Pitch & 0.9 \\
\hline Slice thickness (mm) & 1.0 \\
\hline Increment (mm) & 0.7 \\
\hline Collimation (mm) & $16 \times 0.75$ \\
\hline Tube voltage (kVp) & 90 \\
\hline Current-time product (mAs) & 20 \\
\hline CTDIvol (mGy) & 0.7 \\
\hline Dose-Length Product (mGy cm) & 12.1 \\
\hline
\end{tabular}

All CTs of CF subjects were made supine with free-breathing. Volumetric end-inspiratory and -expiratory acquisitions were obtained using a Philips Brilliance $16 p$ scanner.

See also Kuo et al. Ped Pulmonol 2017

Supplemental Table S2. $\beta$ values before and after adjustment for age in the regression model

\begin{tabular}{llcl}
\hline & UNADJUSTED $\boldsymbol{\beta}$ & ADJUSTED $\boldsymbol{\beta}$ & $\boldsymbol{\Delta} \boldsymbol{\beta}$ (\%) \\
\hline FEV1 & 0,0004741 & 0,0004362 & 8.0 \\
\hline PRAGMA CT score & $-0,004069$ & $-0,003815$ & 6.2 \\
BMI & 0,001398 & 0,001423 & 1.8 \\
\hline Pulmonary exacerbations & $-1,491 \times 10^{-4}$ & $-1,443 \times 10^{-4}$ & 3.2 \\
\hline
\end{tabular}




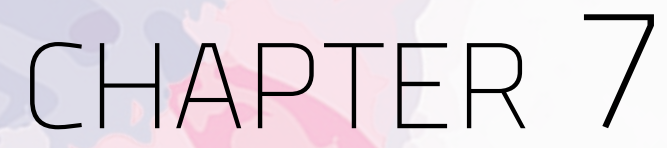

\section{CHARACTERIZING RESPONSES TO CFTR-MODULATING DRUGS USING RECTAL ORGANOIDS DERIVED FROM SUBJECTS WITH CYSTIC FIBROSIS}

Johanna F. Dekkers, Gitte Berkers, Evelien Kruisselbrink, Annelotte Vonk, Hugo R. de Jonge, Hettie M. Janssens, Inez Bronsveld, Eduard A. van de Graaf, Edward E.S. Nieuwenhuis, Roderick H.J. Houwen, Frank P. Vleggaar, Johanna C. Escher, Yolanda B. de Rijke, Christof J. Majoor, Harry G.M. Heijerman, Karin M. de Winter - de Groot, Hans Clevers, Cornelis K. van der Ent, Jeffrey M. Beekman. 


\section{ABSTRACT}

Identifying subjects with cystic fibrosis (CF) who may benefit from cystic fibrosis transmembrane conductance regulator (CFTR)-modulating drugs is time-consuming, costly, and especially challenging for individuals with rare uncharacterized CFTR mutations. We studied CFTR function and response to two drugs---the prototypical CFTR potentiator VX-770 (ivacaftor/kalydeco) and the CFTR corrector VX-809 (lumacaftor)---in organoid cultures derived from rectal epithelia of subjects with CF, who expressed a broad range of CFTR mutations. We observed that CFTR residual function and responses to drug therapy depended on both the CFTR mutation and the genetic backtround of the subject. In vitro drug responses in rectal organoids positively correlated with published outcome data from clinical trials with VX-809 and VX-770, allowing us to predict from preclinical data the potential for CF patients carrying rare CFTR mutations to respond to drug therapy. We demonstrated proof-of-principle by selecting two subjects expressing an uncharacterized rare CFTR genotype (G1249R/ F508del) who dshowed clinical responses to treatment with ivacaftor, and one subject (F508del/R347P) who showed a limited response to drug therapy both in vitro and in vivo. These data suggest that in vitro measurements of CFTR function in patientderived rectal organoids may be useful for identifying subjects who would benefit from CFTR-correcting treatment, independent of their CFTR mutation. 


\section{INTRODUCTION}

Cystic fibrosis (CF) affects about 85,000 persons worldwide, and is caused by mutations in the cystic fibrosis transmembrane conductance regulator (CFTR) gene that encodes an epithelial anion channel. ${ }^{1}$ Nearly 2000 CFTR mutations have been identified (www. genet.sickkids.on.ca), which associate with a wide spectrum of phenotypes (www. CFTR2.org), including CF or milder single-organ CFTR-related diseases. ${ }^{2,3}$ CFTR mutations are classified into six classes according to their effect on CFTR protein expression and function: (i) no synthesis, (ii) impaired trafficking, (iii) defective channel gating, (iv) altered conductance, (v) reduced amounts of functional CFTR, and (vi) impaired cell surface stability of the protein. ${ }^{1}$ Although the clinical relevance and CFTR-based treatment options have been well characterized for common CFTR mutations expressed by large groups of subjects, this is not the case for most of the rare orphan mutations expressed by only a few patients (www.CFTR2.org). ${ }^{2}$

Pharmacotherapy targeting the mutant CFTR protein has been successfully developed for a limited number of CFTR mutations. The CFTR potentiator VX-770 (ivacaftor/ kalydeco) has been registered for treating (F caused by G551D, S1251N and seven other CFTR gating mutations, which are carried by $\sim 5 \%$ of all people with CF.$^{4-6}$ Recent studies indicated that VX-770 combined with the CFTR corrector drug VX-809 (lumacaftor; the combination is termed orkambi) has some beneficial effects on lung function and decreases pulmonary exacerbations in CF subjects homozygous for the most common CFTR mutation F508del (about 45 to $50 \%$ of subjects). ${ }^{7,8}$ Conventional clinical trials to identify drug-responsive subjects among about $50 \%$ of subjects who express mutations other than CFTR-F508del are costly, time consuming, and not possible for those with extremely rare CFTR mutations. New cost-effective methods may help to rapidly extend available CFTR-targeting drugs to subjects with CFTR mutations that are currently not registered for treatment.

Here, rectal organoid cultures derived from the rectal epithelia of 71 individuals expressing 28 different CFTR genotypes (see Table S1 for an overview of CFTR genotypes and mutation classification) were used to study residual CFTR function and drug-modulated CFTR function..$^{9,10}$ Rectal organoid cultures are three-dimensional (3D) primary stem cell cultures that self-organize into tissue-recapitulating "mini-guts" in vitro that enable the long-term expansion and biobanking of primary patient tissue using defined growth conditions.10 Here, we used a recently established forskolin-induced swelling (FIS) assay $(11,12)$ and developed a new assay that measures the steady-state lumen area (SLA) of organoids in the absence of forskolin. ${ }^{11,12}$ Data confirmed that the FIS assay was fully dependent on CFTR as previously reported by us and others. ${ }^{12-14}$ The FIS assay enabled sensitive measurements of CFTR function, allowing the study of residual CFTR function among CF patients an non-CF controls. The CFTR-dependent SLA assay facilitated the comparison of CFTR function among CF patients and non-CF controls. Rectal organoids 
were obtained independent of age and with only limited discomfort. ${ }^{15,16}$ We correlated in vitro responses by rectal organoids to VX-809 and VX-770 with published clinical trial data and have provided proof of principle that subjects with CF carrying rare CFTR mutations can be selected for treatment with VX-770 on the basis of data from patientderived rectal organoids.

\section{METHODS}

\section{Study design}

Residual CFTR function and responses to the CFTR corrector drug VX-809 (Lumacaftor) and CFTR potentiator drug VX-770 (ivacaftor / KALYDECO) in rectal organoids derived from 71 subjects expressing wild-type or mutant CFTR were measured using two assays: the FIS and the SLA assay. Quantification of the SLA was performed in a blinded fashion. As proof of principle for organoid-based selection of in vivo drug responders or nonresponders, subjects with a rare CFTR mutation and either a high (F508del/ G1249R, $N=2$ ) or low (F508del/R347P, $N=1$ ) organoid response to VX-770 in vitro were assessed in an open-label trial with KALYDECO, dosed according to the manufacturer's instructions. Four weeks of treatment was followed by a 4-week washout period. Clinical outcome parameters were measured at baseline, after treatment, and after washout. These included the sweat chloride concentration (SCC), nasal potential difference (NPD), body weight, forced expiratory volume in one second $\left(\mathrm{FEV}_{1}\right)$, airway resistance (RAW 0.5 ) and cystic fibrosis questionnaire-revised (CFQ-r).

\section{Human material}

The Ethics Committee of the University Medical Centre Utrecht and Erasmus Medical Centre Rotterdam approved this study, and informed consent was obtained from all participating subjects. Organoids were generated from rectal biopsies after they were used for intestinal current measurements (ICM), which were performed for (i) standard care, (ii) voluntary participation in studies or (iii) diagnosis.

\section{Crypt isolation and organoid culture from rectal suction biopsies}

Methods were slightly adapted from protocols described previously., ${ }^{9,12}$ In short, crypts were isolated and seeded in 50\% Matrigel (growth factor-reduced and phenol-free; BD bioscience) in 24-well plates ( 10 to 30 crypts in three 10- $\mu$ l Matrigel droplets per well). Growth medium was further supplemented with Primocin (1:500; Invivogen). ${ }^{12}$ Vancomycin and gentamicin (both from Sigma) were added during the first week of culture. The medium was refreshed every $2-3$ days, and organoids were passaged $~ 1: 5$ every $7-10$ days. 


\section{The FIS assay}

Methods were slightly adapted from protocols described previously. ${ }^{9,12}$ In short, rectal organoids (passage 1-15) from a 7 to 10-day old culture were seeded in 96-well culture plates (Nunc) in $5 \mu$ l 50\% Matrigel containing 20 to 80 organoids and immersed in $100-\mu l$ medium with or without $3 \mu \mathrm{M}$ VX-809 (Selleck Chemicals LLC). One day after seeding, organoids were incubated for 30 min with $3 \mu \mathrm{M}$ calcein green (Invitrogen), stimulated with forskolin with or without $3 \mu \mathrm{M}$ VX-770 (Selleck Chemicals LLC), and directly analyzed by confocal live cell microscopy (LSM710, Zeiss) (everything was performed at $37^{\circ} \mathrm{C}$ ). The total organoid area (xy plane) increase relative to $t=0$ of forskolin treatment was quantified using Volocity imaging software (Improvision). Occasionally, cell debris and unviable structures were manually excluded from image analysis based on criteria described in detail in a standard operating procedure (SOP). The area under the curve (AUC) ( $\mathrm{t}=60 \mathrm{~min}$; baseline, 100\%) was calculated using Graphpad Prism.

\section{The SLA assay}

Organoids were seeded as described above; incubated for 24 hours with dimethyl sulfocide (DMSO), $3 \mu \mathrm{M}$ VX-809, $3 \mu \mathrm{M}$ VX-770, or their combination; labeled with calceingreen; and analyzed by confocal microscopy (all at $37^{\circ} \mathrm{C}$ ). The total area (xy plane) of all organoids in a well was automatically quantified using Volocity, and the luminal area of all organoids of the same well was marked manually and calculated using Volocity in a blinded fashion. The SLA was expressed as the luminal organoid surface area of the total organoid surface area in percentage (Fig. 1b). Occasionally, cell debris and nonviable structures were excluded similarly to the FIS assay.

\section{ICM}

Transepithelial chloride secretion in human rectal biopsies was measured using slight adaptations of the procedures described previously. ${ }^{17,18}$ See the supplementary materials for a detailed description.

\section{Measurement of SCC and NPD}

Both SCC and NPD measurements were performed according to the most recent version of the SOP operating procedure of the European Cystic Fibrosis Society Clinical Trials Network (ECFS-CTN).

\section{Statistical analysis}

Results are presented as means \pm standard deviation (SD) or means \pm standard error of the mean (SEM), with the number of biological and technical replicates indicated per figure. Statistical analysis was performed by unpaired two-tailed Student's t test, or 
by Pearson correlation using GraphPad Prism software. The 95\% confidence level was considered significant.

\section{RESULTS}

\section{The SLA of rectal organoids derived from patients with CF and from healthy controls}

We observed that rectal organoids from healthy subjects were phenotypically different from rectal organoids derived from subjects expressing two CFTR mutations (which have been established as (F-causing) under standard culture conditions, in the absence of an additional CFTR activator such as forskolin (Fig. 1a). The various mutations are associated with different disease severities (for example, F508del > A455E > R117H-7T) (www.CFTR2.org). We quantitated the SLA by measuring the lumen area as a percentage of total organoid area (Fig. 1b and supplemental Fig. S1a). The SLA discriminated between healthy controls (fluid-filled/cystic phenotype; 35 to 70\%; wild-type/wild-type, 51\% \pm 10 ; wild-type/F508del,47\% \pm 11 ; Mean \pm SD) and class I to $V$ mutant CFTR organoids (non- to low fluid-filled/cystic phenotype, 0-10\%) at the individual level. In CF organoids, the SLA only discriminated between class I to III and class IV and V mutant organoids at the group level [class I to III (1.3 \pm 1.4$)$ versus class IV and V (5.0 \pm 3.3 ) (means $\pm S D) ; P<0.0001)$ (Fig. $1 c$ and supplemental Fig. S1b).

Paired analysis of FIS and SLA assays measuring a single healthy control or single F508del/ $\mathrm{R} 117 \mathrm{H}-7 \mathrm{~T}$ mutant rectal organoid indicated that a large SLA (> 40\%) was negatively associated with the increase in relative area of rectal organoids upon stimulation with forskolin $(R=-0.7206$ and $p<0.0001) .{ }^{12}$ In contrast, FIS assay measurements were unaffected for organoids having SLA values between 0 and $40 \%$ ( $R=0.0812, p=$ 0.4569; Fig. 1d). Because healthy control cultures contained many organoids with a large SLA (Fig. 1C,d and supplemental Fig. S1b), FIS rates in healthy control organoids were negatively affected by their SLA phenotype (Fig. 1d and supplemental Fig. S1C), leading to underestimation of wild-type CFTR function by the FIS as indicated by higher FIS of rectal organoid cultures carrying CFTR mutations with high residual function (for example $\mathrm{R} 117 \mathrm{H})$. Rectal organoids from CF subjects were all within an average SLA phenotype that did not affect FIS (all below 10\%; Fig. 1c and supplemental Fig. S1b), allowing direct comparison of FIS among rectal organoids CF subjects. Together these data indicate that (i) SLA is a CFTR-dependent phenotype that discriminates between healthy controls and subjects with CF, (ii) FIS rates can be compared among CF rectal organoids, and (iii) FIS rates cannot be compared between healthy control and mutant CFTR organoids because of high SLA in healthy control cultures. 


\section{Forskolin-induced residual CFTR function in rectal organoids from CF subjects}

We assessed the swelling of rectal organoids derived from 37 individuals expressing various class I to V CFTR mutations using eight different forskolin concentrations 10.008 to $5 \mu \mathrm{M}$ ) to maximize the dynamic range of the assay. As described previously, rectal organoid swelling was calculated from 60 -min time tracings of the surface area increase relative to $\mathrm{t}=0$ (Fig. 2a). ${ }^{12}$ We observed a forskolin dose-dependent increase in swelling that greatly varied among organoids with different CFTR mutations (Fig. 2b). Swelling at $0.8 \mu \mathrm{M}$ forskolin was used to compare different genotypes (Fig. 2C) or individuals with clear detectable residual CFTR function (Fig. 2d), indicating the potential to discriminate between residual CFTR function of organoids expressing different or identical CFTR mutations (Fig. 2c,d). At $0.8 \mu \mathrm{M}$ forskolin, the residual CFTR function of organoids derived from individual donors correlated best with paired ex vivo ICM $(R=0.5109$ and $P=$ 0.0055 ; Fig. 2e) and the in vivo SCC ( $\mathrm{R}=-0.7011$ and $\mathrm{P}<0.0001$; Fig. $2 f)$ compared to the residual CFTR function detected at other forskolin concentrations (Fig. 2b). 19,17,20-22 Swelling of several rectal organoid cultures expressing severe CFTR genotypes (but not 1811+1G>C/1811+1G>C and 166delTA/3120G >A) were most optimally detected at saturating forskolin concentrations ( $5 \mu \mathrm{M}$; Fig. 2b and supplemental Fig. S2). Comparisons of residual CFTR function as measured by the FIS or SLA assays indicated that the SLA assay less clearly discriminated among individuals (Fig. $2 d$ and supplemental Fig. S1b) and genotypes (Supplemental Fig. S1d) than did the FIS assay. Some variation in the SLA phenotype appeared independent of CFTR function. For instance, 1811+1G >C/1811+1G>C CF organoids had a relatively large SLA, whereas FIS and thus CFTR function was absent in these cultures (Supplemental Fig. S1, b and c). These data suggest that the SLA assay has a dynamic range at higher CFTR activity compared to the FIS assay (Fig. 2 and supplemental Fig. S1).

Thus, rectal organoid swelling could be used to quantitate individual residual CFTR function over a large dynamic range that correlated with known in vivo and ex vivo CFTRdependent markers.

\section{Pharmacological restoration of CFTR function in rectal organoids}

Next, we studied correction of FIS by VX-809 and VX-770 in rectal organoids (see supplemental Fig. S3 for a detailed analysis). Mutant rectal organoids devoid of any residual function $(1811+1 \mathrm{G}>\mathrm{C} / 1811+1 \mathrm{G}>\mathrm{C}$ and 166delTA/3120G $>A$ ) did not show druginduced swelling, indicating expression of mutant CFTR that do not respond to VX-770 and VX-809. All other CFTR genotypes responded in a forskolin dose-dependent manner to the drug treatments, and genotype-specific profiles were observed (examples are shown in Fig. 3a, and all genotypes are in supplemental Fig. S4a). VX-809 treatment modestly increased FIS for most genotypes (most likely through the CFTR-F508del mutation), but a greater effect in A455E-expressing organoids suggested a potential trafficking defect 
Figure 1. The SLA of rectal organoids from CF patients or healthy controls.

a.

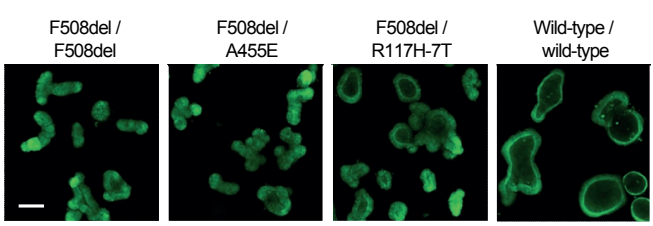

b.

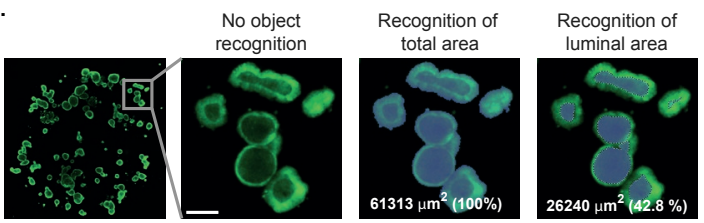

C. Example of steady-state lumen area (SLA) calculation from 3 experiments (Exp.)
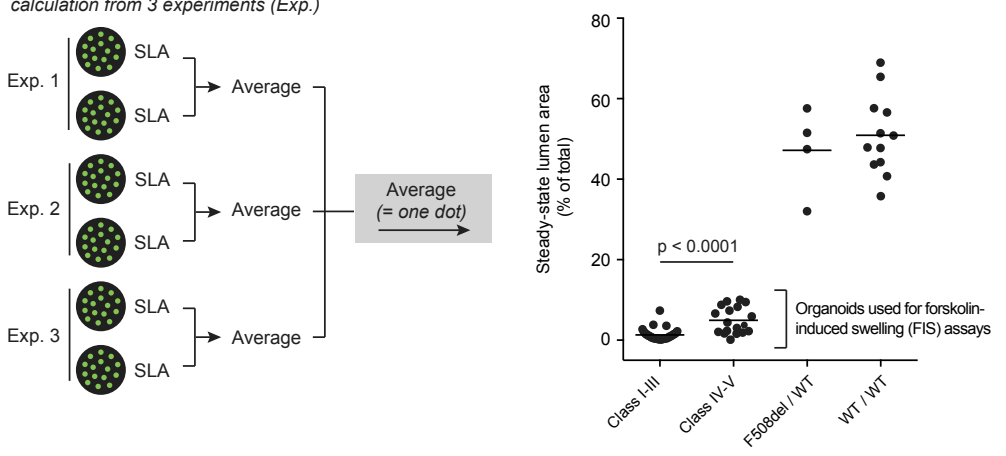

d.
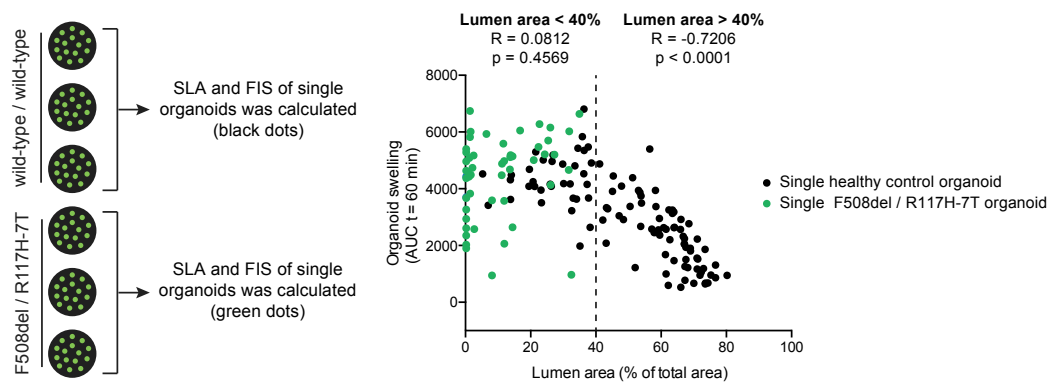
(a) Representative confocal images of calcein-green-labeled rectal organoids from patients with different CFTR mutations or healthy controls [wild-type (WT)] using standard culture conditions. Scale bar $100 \mu \mathrm{m}$.

(b) Quantification of the SLA. Shown is recognition of the total lumen area of rectal WT/WT organoids (xy plane) by Volocity imaging software. The luminal surface area (in $\mu \mathrm{m} 2$ and \%) of the total organoid surface area $(100 \%)$ is indicated at the bottom of the image. Scale bar $80 \mu \mathrm{m}$.

(c) Quantification of the SLA of rectal organoids derived from individuals expressing two CFTR class I-III ( $n=35$ ) or class IV-V ( $n=18)$ mutations, or from healthy control subjects either expressing WT/WT (WT; $n=12$ ) or F508del/WT CFTR $(n=4)$. (See Table 11 for the classification of different CFTR mutations and supplemental Fig. S1b for the responses per subject). 2-5 independent experiments were performed for each subject. Data were generated over a period of 2 years.(d) Paired analysis of measurements for the FIS and SLA assays for rectal organoids from a single healthy control and from a CF individual with the F508del/R117H mutation. The data from the control and CF rectal organoids were derived from three independent wells of the same experiment.

for this mutant CFTR (Fig. 3a and supplemental Fig. S4a). Incubation with only VX-770 greatly enhanced FIS in organoids that expressed known class III to $\mathrm{V}$ mutations on at least one allele but only modestly enhanced FIS in organoids expressing class I/class II or class I//class II genotypes. Together, VX-809 and VX-770 synergistically increased FIS in organoids homozygous for the F508del mutation or compound heterozygous for F508del and a class I mutation (N1303K, 711-1G>T, R347P or A455E). In contrast, VX-770 combined with VX-809 only had minor effects on FIS as compared to VX-770 alone for all other genotypes (Fig. 3a and supplemental Fig. S4a). These data indicated that the CFTR genotype determined the qualitative response to these CFTR-targeting drugs.

To identify an optimal approach for estimating in vivo drug efficacy, we assessed correlations between currently available clinical trial data (summarized in Fig. 3b) and drug-induced FIS for rectal organoids quantitated in different ways [per concentration of forskolin, or as the AUC of a forskolin dose range; see supplemental Fig. S4b for DMSOcorrected responses of the clinically assessed CFTR genotypes].6,23,24 Because drugtreated organoids expressing the $\mathrm{S} 1251 \mathrm{~N}$ or $\mathrm{R} 117 \mathrm{H}-7 \mathrm{~T}$ mutations approached maximal FIS rates (AUC, 3500) at higher forskolin concentrations (Fig. 2a and supplemental Fig. S4a), their DMSO-corrected drug responses declined at forskolin concentrations higher than $0.128 \mu \mathrm{M}$ (Supplemental Fig. S4b) leading to underestimation of drug treatment effects. The strongest positive correlation was observed between the response to therapy in organoids at $0.128 \mu \mathrm{M}$ forskolin (Fig. 3c) and the in vivo absolute change in percent predicted FEV1 ( $R=0.8706, P=0.0240$, Fig. 3d;) compared to the drug-induced FIS detected at other forskolin concentrations. Comparable results were obtained when drug-induced FIS at $0.128 \mu \mathrm{M}$ forskolin was correlated with the relative change in percent predicted FEV1 $(R=0.8949, P=0.0402, n=5)$ because no relative data were available for the S1251N mutation subgroup; (Supplemental Fig. S5,a and b).

On the basis of these data, we constructed a colored reference map to visualize the clinical potential of CFTR-targeting therapy for individuals with CFTR genotypes with unknown in vivo treatment efficacy (Fig. 3c). This map suggested the potential for clinical responses with $V X-770$ or combined $V X-809+V X-770$ treatment for subjects 
Figure 2. Residual CFTR function in rectal organoids measured by FIS.

a.

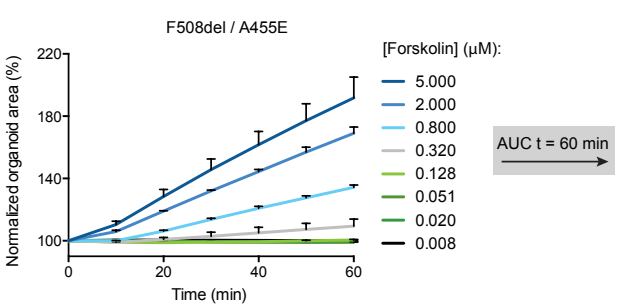

d.

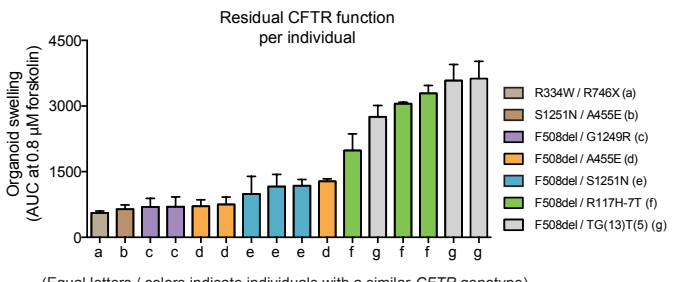

(Equal letters / colors indicate individuals with a similar CFTR genotype) b.

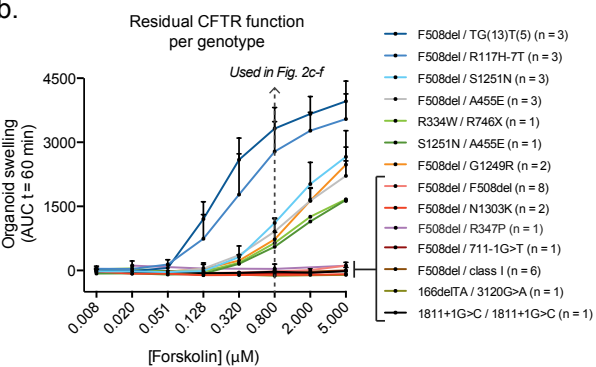

e.

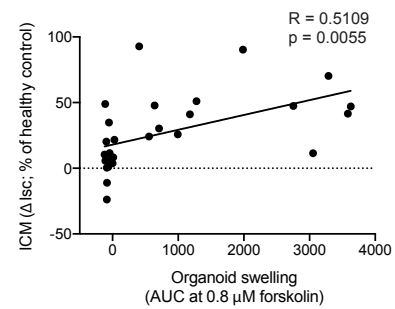

(a) Quantification of the surface area relative to $\mathrm{t}=0$ (normalized area) of F508del/A455E mutant rectal organoids at different forskolin concentrations averaged from two independent wells. Data are means \pm SD.

(b) FIS of rectal organoids with various mutations expressed as the absolute AUC calculated from tracings comparable to (a) (baseline, 100\%, $\mathrm{t}=60 \mathrm{~min}$ ). (The data are similar to the residual CFTR function data presented in supplemental Fig. S4a). Data are means \pm SD.

(c and d) Rectal organoid swelling at $0.8 \mu \mathrm{M}$ forskolin shown as multiple individuals per CFTR genotype (c) or per individual (d). Data are means \pm SD (c) or means \pm SEM (d).

(e and f) Pearson correlations of ICM (e) or measurements of SCC (f) versus rectal organoid swelling at $0.8 \mu \mathrm{M}$ forskolin. ICM values were normalized to healthy control responses to correct for the use of two different protocols. SCC values were obtained from the Dutch registry database. Each dot represents one individual. (b to d) Data were generated over a period of 1.5 years; $80 \%$ of data were generated during a period of 4 months using a single batch of complete growth medium to limit technical variability. n, number of subjects. Each subject was measured at two to five independent time points in duplicate. The class I mutations included: G542X, R1162X (two different subjects), W1282X, DELE2.3 and E60X. Isc, short circuit current. 
C.

Residual CFTR function per genotype

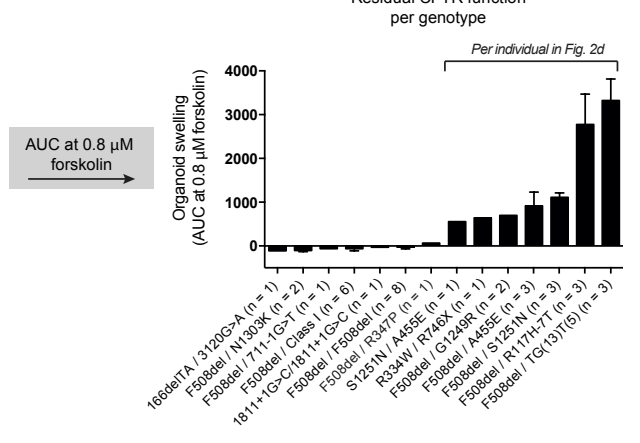

f.

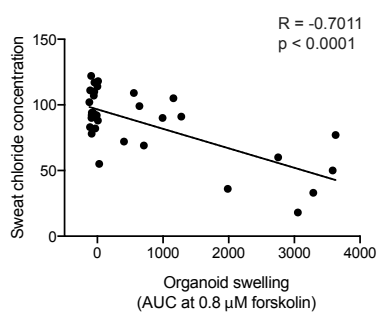

expressing G1249R and TG(13)T(5) mutations, VX-809 + VX-770 for subjects expressing the A455E mutation, and modest potential of VX-809 + VX-770 for the individual who was compound heterozygous for R334W and R746X. Furthermore, CFTR-N1303K, CFTR711-1G>T and CFTR-R347P mutations are likely to be nonresponsive CFTR mutations, given that the average responses of F508del/N1303K, F508del/711-1G>T, or F508del/ R347P rectal organoids were not higher than those of F508del/class I organoids (Fig. 3c). The FIS responses to VX-809 and VX-770 for each individual were variable among donors with different or identical CFTR mutations, indicating that both the CFTR mutations and an individual's genetic background modified the response to drug therapy (Fig. 3e). Correlations between individual residual CFTR function and response to $\mathrm{VX}-770(\mathrm{R}=$ 0.8030; $P<0.0001), V X-809(R=0.0113 ; P=0.9495)$, or both $(R=0.5294 ; P=0.0013)$ indicated that response to $\mathrm{VX}-770$ correlated most strongly with residual CFTR function (Supplemental Fig. S6). 
Figure 3. Pharmacological repair of CFTR function in rectal organoids measured by FIS.

a.

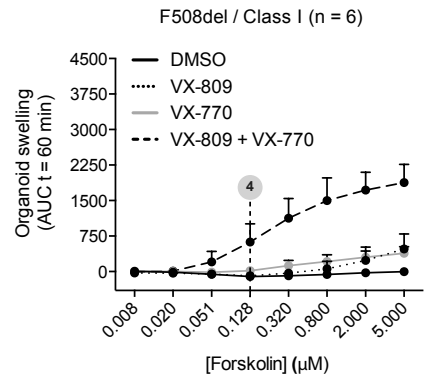

F508del / S1251N ( $=3)$

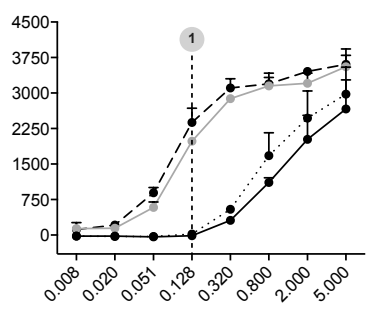

[Forskolin] $(\mu \mathrm{M})$
F508del / F508del $(n=8)$

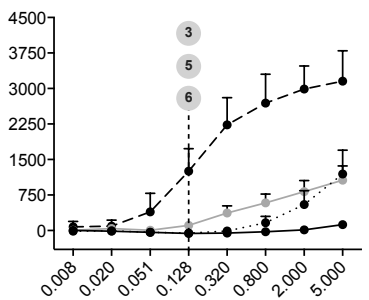

[Forskolin] $(\mu \mathrm{M})$

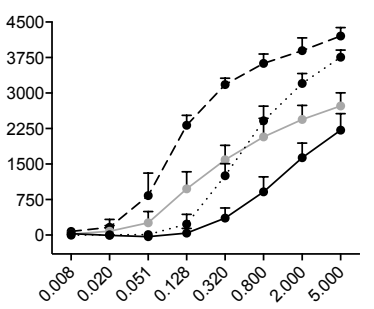

[Forskolin] $(\mu \mathrm{M})$
F508del / R117H-7T $(n=3)$

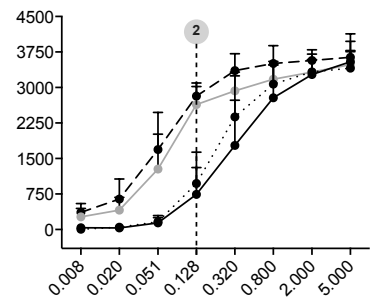

[Forskolin] ( $\mu \mathrm{M})$

b.

\begin{tabular}{|c|c|c|c|c|c|}
\hline & Treatment & Phase & CFTR genotype & $\begin{array}{l}\text { Absolute change } \\
\% \text { predicted } \mathrm{FEV}_{1} \\
\text { versus placebo }\end{array}$ & Reference \\
\hline & VX-770 & III & S1251N / other & $8.7 \%(p<0.0001)$ & 6 \\
\hline 2 & VX-770 & III & R117H-5T/7T / other & $5.0 \%(p=0.01)$ & investors.vrtx.com \\
\hline 3 & VX-809 + VX-770 & III & F508del / F508del & $3.3 \%(p<0.001)$ & 8 \\
\hline 4 & $V X-809+V X-770$ & ॥ & F508del / other & $0.3 \%$ (NS) & 7 \\
\hline & VX-770 & ॥ & F508del / F508del & $1.7 \%$ (NS) & 22 \\
\hline 6 & VX-809 & Ila & F508del / F508del & $0.27 \%$ (NS) & 23 \\
\hline
\end{tabular}

(a) Rectal organoids expressing various CFTR mutations were treated as indicated DMSO, vehicle; VX-809, $3 \mu \mathrm{M}$; VX-770, $3 \mu \mathrm{M}$ ] and stimulated with forskolin (see supplemental Fig. S3 for detailed analysis; these figures are also presented in supplemental Fig. S4a). Responses at $0.128 \mu \mathrm{M}$ forskolin (dotted line) were used for correlations with clinical trial data in (b). The different numbers define specific CFTR genotypes and treatments, and they associate with the numbers $1-6$ of panels (b) to (d). Data are means \pm SD.

(b) Data overview of clinical trials with CFTR-correcting treatments in subjects expressing different CFTR mutations. In each clinical trial, results of the most optimal treatment strategy are presented. For the R117H trial, only data from CF subjects aged $>18$ were used, because subjects aged 6 to 18 had a different mean baseline FEV1 compared to those in the other trials. The numbers correlate with the numbers in (a), (c), and (d). NS, not significant. 


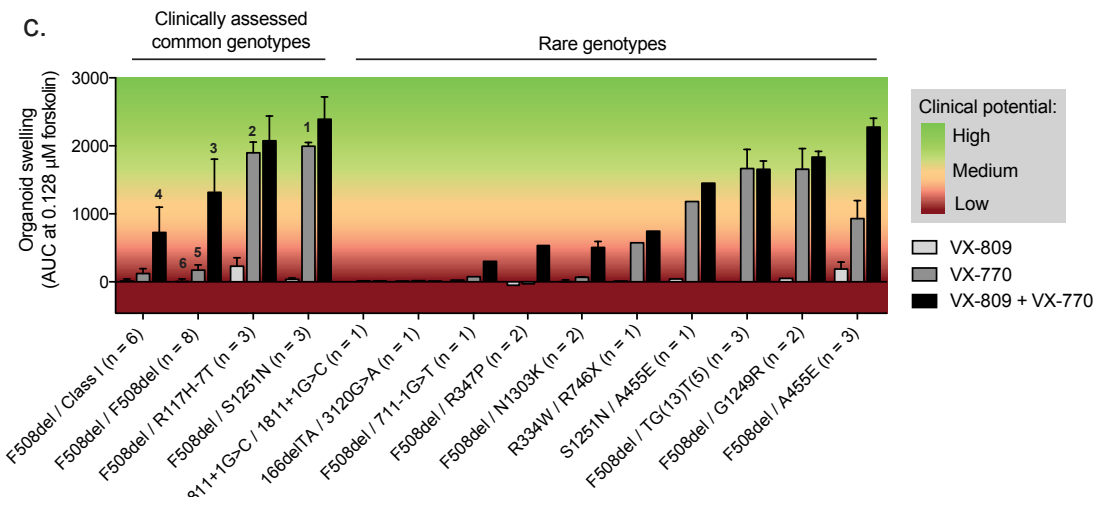

d.

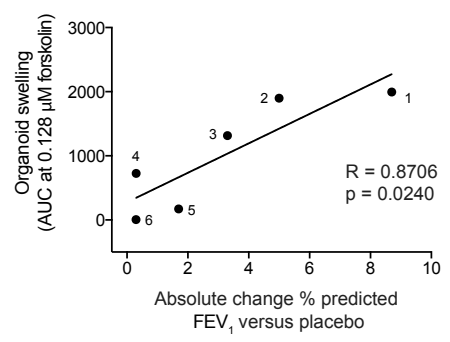

e.

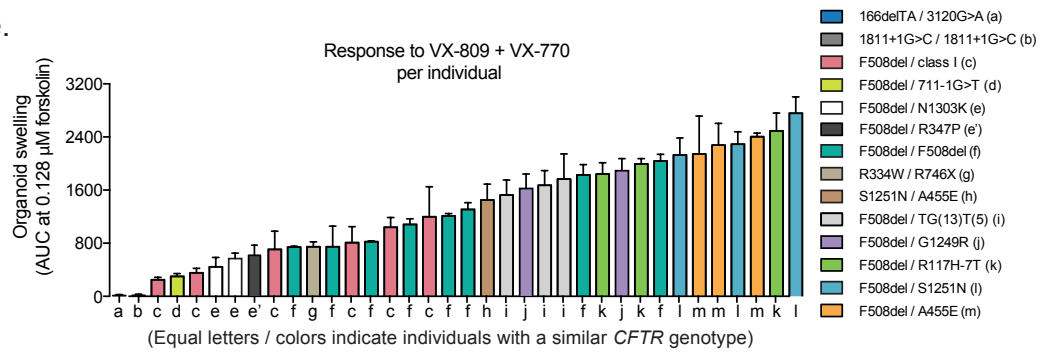

(c) CFTR modulator-corrected swelling at $0.128 \mu \mathrm{M}$ forskolin (AUC at $\mathrm{t}=60 \mathrm{~min}$ ) corrected for the DMSO condition presented per CFTR genotype. These DMSO-corrected responses were calculated from data presented in (a) and supplemental Fig. S4a. The numbers indicate the responses that represent CFTR genotypes and treatments that have been clinically assessed and are associated with the numbers in (a), (b) and (d). The color profile was based on the clinical effectiveness of studies presented in (b). Data are means \pm SD.

(d) Pearson correlation of drug-corrected rectal organoid swelling [results from (c)] versus lung function increase [results from (b)]. The numbers are associated with (a) to (c).

(e) The VX-809 + VX-770-corrected swelling of rectal organoids at $0.128 \mu \mathrm{M}$ forskolin (AUC at $\mathrm{t}=60 \mathrm{~min}$ ) corrected for the DMSO control presented per individual. These DMSO-corrected responses were calculated from data presented in (a) and supplemental Fig. S4a. Data are means \pm SEM. Data were generated over a period of 1.5 years; $80 \%$ of the data were generated during a period of 4 months using a single batch of complete growth medium to limit technical variability. n, number of subjects. Each subject was measured at two to five independent time points in duplicate. The class I mutations included: G542X, R1162X (two different subjects), W1282X, DELE2.3 and E60X. 
Compared to the FIS assay, essentially similar data were generated when the forskolinindependent SLA assay was used to measure drug effects after a 24-hour incubation with VX-770, VX-809, or a combination of the two (Fig. 4a to C). In contrast to the FIS assay, the SLA assay allowed comparisons between CF and healthy control rectal organoids (Fig. 4b). The SLA of drug-treated organoids reached 10 to $120 \%$ of that for wild-type healthy control organoids (set at 100\%) (Fig. 4b), depending on the CFTR mutations. In contrast, wild-type healthy control organoids reached 140\% SLA upon overnight incubation with VX-770. The SLA of F508del/wild-type organoids was $80 \%$ (instead of the expected $50 \%$ ) of wild-type healthy control organoids, suggesting a nonlinear relation between SLA and CFTR function at higher CFTR activities. This suggested that drug efficacy expressed as a percentage of wild-type control organoids was somewhat overestimated (Fig. 4b). In line with limited induction of FIS by VX-809 alone at lower concentrations of forskolin (Fig. 3a, supplemental Fig. S4a), VX-809 did not induce SLA in CF organoids of any CFTR genotype in the absence of forskolin; correction of CFTR function was seen with the combined treatment of VX-809 and VX-770 (Fig. 4b,c). These results indicated that the SLA assay was not suitable for detection of a response to VX-809 monotherapy and suggested that channel activity of VX-809-corrected CFTR required higher $3^{\prime}, 5^{\prime}$-cyclic adenosine monophosphate (CAMP) concentrations as compared to VX-770-potentiated CFTR.

The SLA assay recapitulated the relationship between the CFTR genotype and responses to $\mathrm{VX}-770$ or $\mathrm{VX}-809+\mathrm{VX}-770$ as observed by the FIS assay (Fig. 3c versus Fig. 4C). A significant correlation between drug-induced SLA and an increase in absolute lung function was only observed when data for the R117H CFTR mutation (associated with mild CF disease) were excluded ( $R=0.9690$ and $p=0.0065$; supplemental Fig. S5, $a$ and b). When all six CFTR genotypes were included, we observed a trend for correlation $(R=$ 0.7247 and $\mathrm{P}=0.1032 ;$ Fig. $4 \mathrm{~d}$ and supplemental Fig. S5, $\mathrm{a}$ and $b$ ). This may be because the dynamic range of the SLA assay at high CFTR function prevented correction for residual function associated with CFTR-R117H in the absence of treatment. The drug-induced $S L A$ correlated with a relative increase in lung function $(R=0.9172$ and $P=0.0282, n=5$; supplemental Fig. S5a and b).

Thus, the FIS and SLA assays enabled the characterization of drug-corrected CFTR function in organoids derived from donors expressing class I to $V$ mutations. Responses to drug therapy in the FIS and SLA assays and CFTR genotype were associated with published outcome data from clinical trials with CFTR-correcting drugs. . $^{6,23,24}$ This suggested that our rectal organoid model could be used to identify drug-responsive individuals with rare CFTR genotypes.

\section{CFTR correction in rectal organoids from patients with two F508del mutations}

To further demonstrate that intersubject variability could be consistently measured 
Figure 4. Pharmacological correction of CFTR function in rectal organoids measured by the SLA assay.

a.

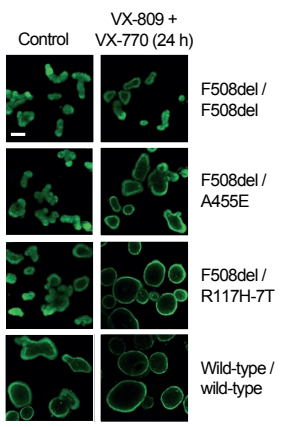

(a) Representative confocal images of calcein green-labeled rectal organoids with or without a 24 hour combination treatment with VX$809+V X-770(V X-809,3 \mu M ; V X-770,3 \mu M)$. Scale bar $100 \mu m$

(b and c) Quantification of the SLA of rectal organoids incubated for 24 hours with drug treatments as indicated, and normalized to the average of the DMSO-treated WT/WT rectal organoids (set at 100\%) per experiment (b) or presented as absolute SLA corrected for DMSO (c). The numbers refer to Fig. 3b. Data were generated over a 5 week time frame with a single batch of complete growth medium to limit technical variability. Data are means \pm SD. n, number of subjects. Each subject was measured at two to five independent time points in duplicate. The class I mutations include G542X, R1162X, and W1282X. (d) Pearson correlation of the drug-induced SLA versus lung function increase [the numbers refer to Fig. 3b, and the results in (c)].

b.

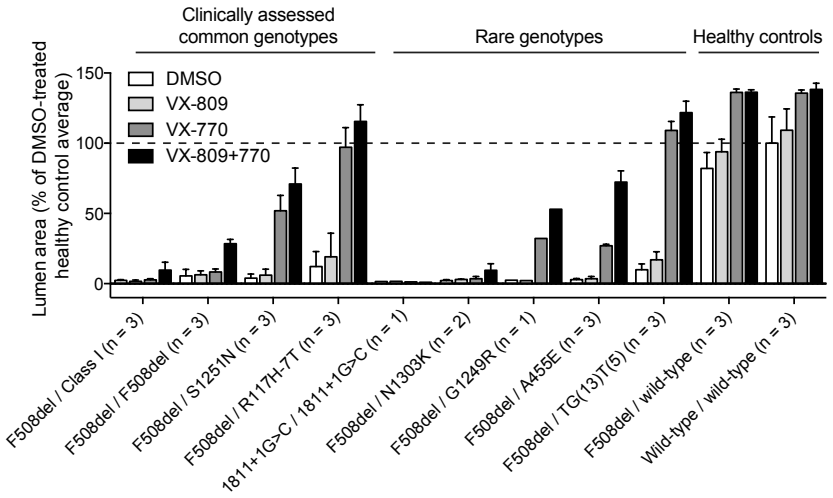

C.
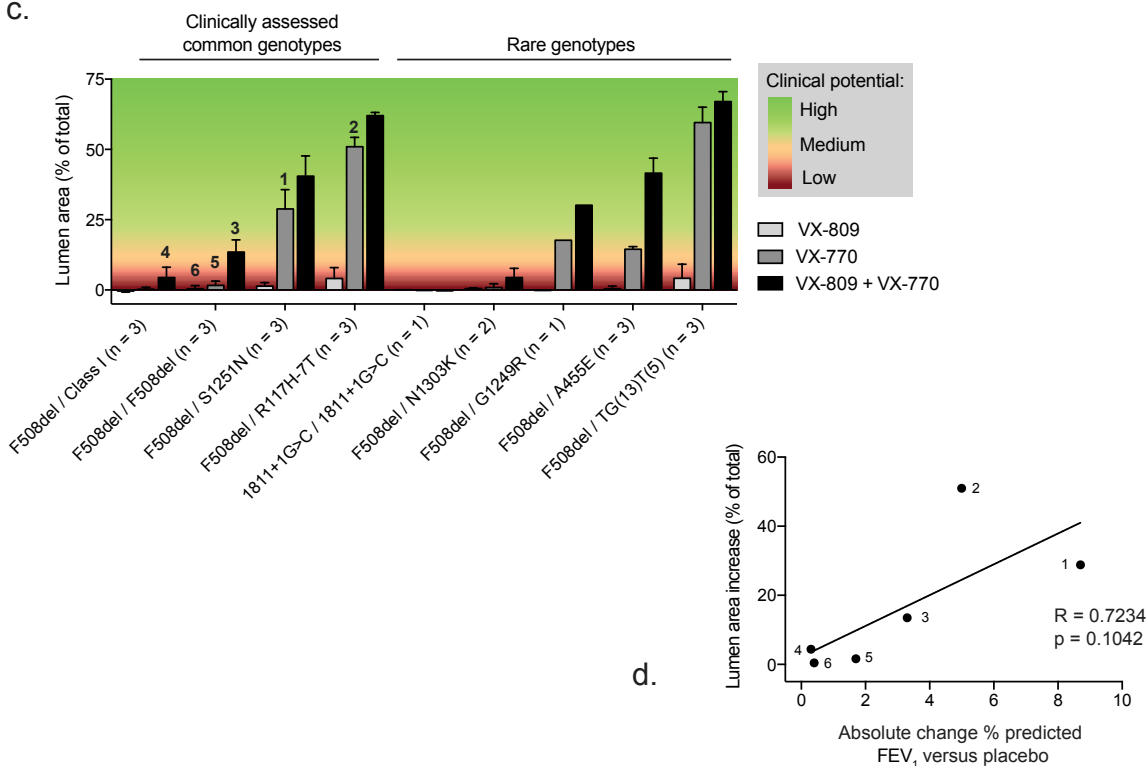
Figure 5. Long-term stability of swelling in F508del homozygous rectal organoids after forskolin treatment.

a.

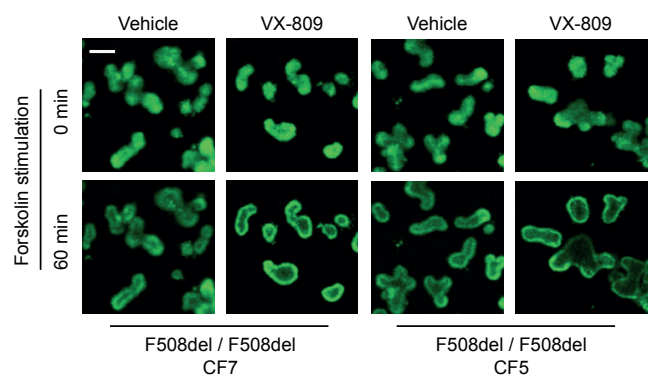

c. F508del / F508del
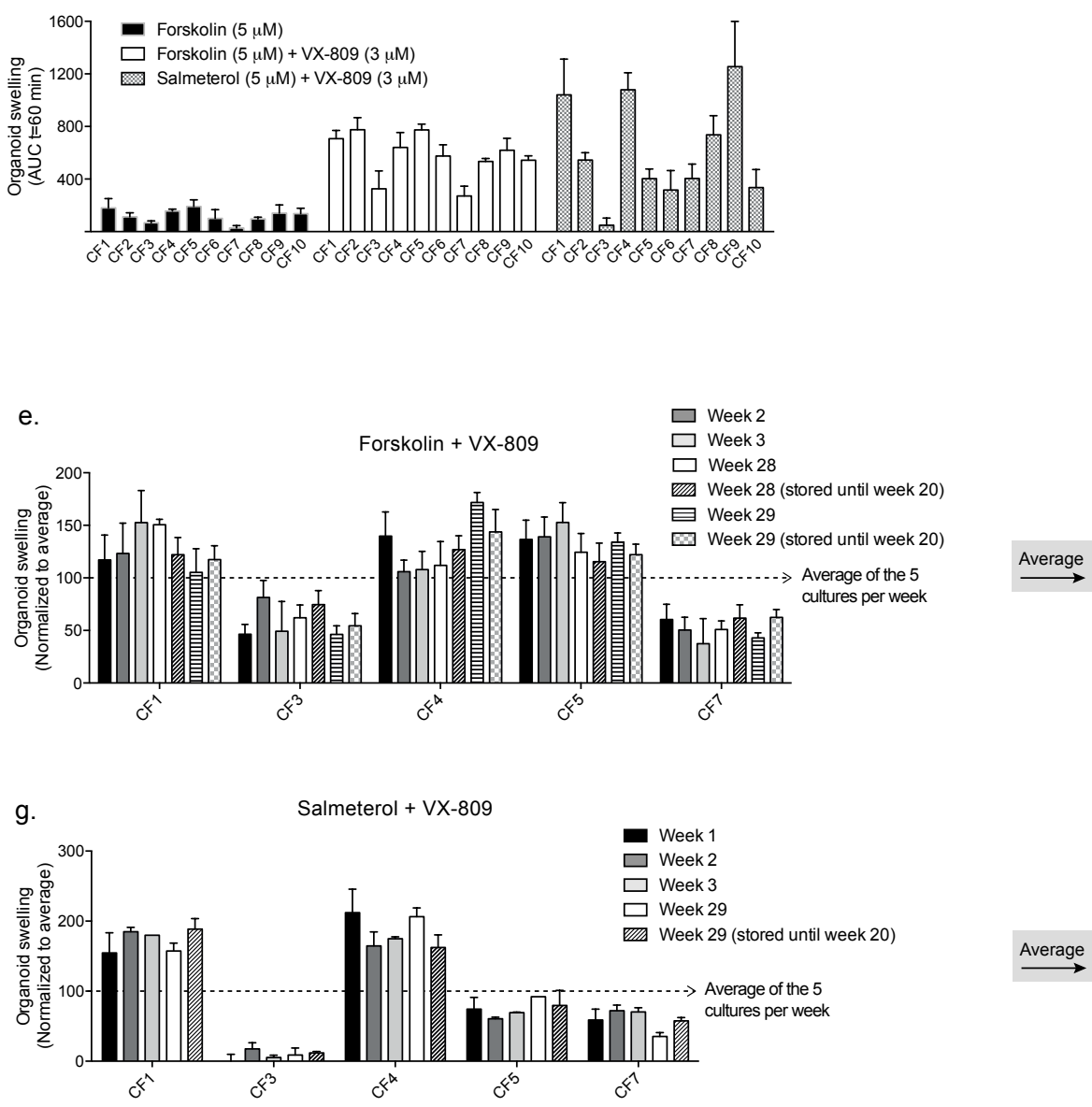
b.

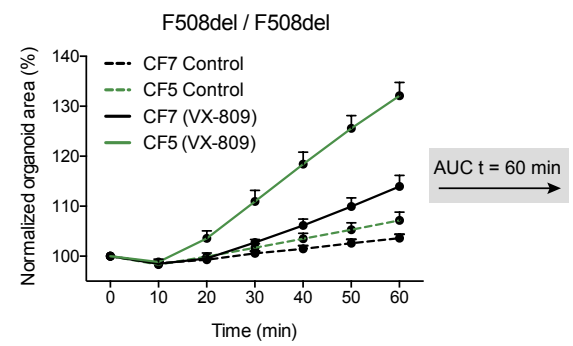

d.

F508del / F508del

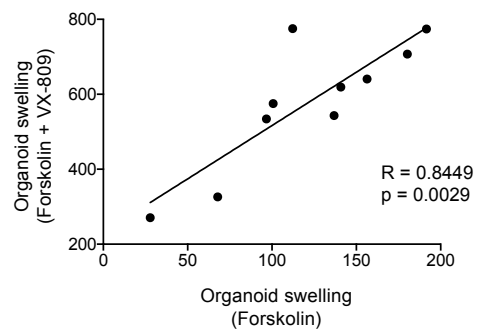

$f$

f.

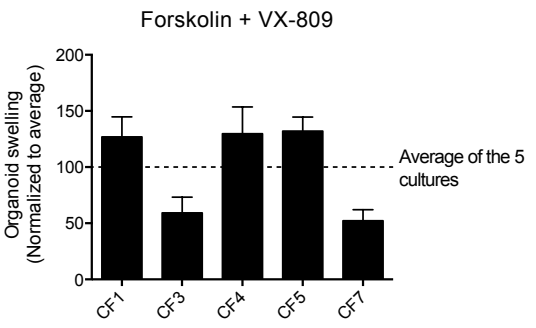

h. Salmeterol + VX-809

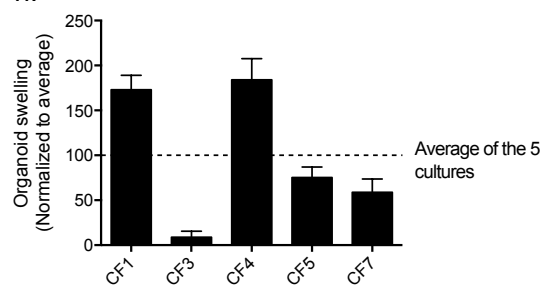

(a and b) Representative confocal images (a) or quantification of the surface area increase relative to $t=0$ (averaged from 3 wells) (b) of calcein green-labeled and forskolin-induced rectal organoids derived from two individuals with the F508del homozygous mutation with or without 24 hours of treatment with VX-809 (3

$\mu \mathrm{M})$. Scale bar $90 \mu \mathrm{m}$. Data are means \pm SD. (c) Swelling of rectal organoids induced by forskolin or rectal organoids preincubated for 24 hours with VX-809 and then induced to swell by forskolin or salmeterol. Data are expressed as the absolute AUC calculated from time tracings comparable to (b) (baseline, $100 \%$, $\mathrm{t}=60 \mathrm{~min}$ ). For each experiment, the cultures were assessed simultaneously to limit technical variation. Responses were averaged from three independent experiments performed at weekly interval [weeks 1 to 3; see supplemental Fig. $\mathrm{S} 7$ (a to c) for the results of the independent experiments].

(d) Pearson correlation for the uncorrected or VX-809 (3 $\mu \mathrm{m})$-corrected FIS from (c).

(e to h) After the first three experiments [weeks 1 to 3; averages are presented in (c)], two lowforskolin-responding and three high-forskolinresponding rectal organoids were maintained in culture and measured again at weeks 28 and 29 , or they were thawed from liquid nitrogen storage at week 20 and measured at weeks 28 and 29. Data on swelling of VX-809-corrected rectal organoids in response to forskolin (e and f) or salmeterol ( $g$ and h) are presented per experiment (e to g) or as an average ( $f$ to h). The swelling responses are normalized to the average response of the five cultures per experiment (100\%). [See supplemental Fig. S7 (e to h) for the absolute responses.] In (e) and (g), the SD represents variation from three independent wells; in ( $f$ ) and (h), the SD represents variation between different experiments. Data are means \pm SD. 
among donors independent of their CF-causing mutations, we assed swelling of 10 F508del homozygous rectal organoids in response to forskolin or various $\beta_{2}$-agonists using identical assay and culture conditions to minimize the impact of technical variation (Fig. 5 and supplemental Fig. S7). $\beta_{2}$-agonists activated CFTR via $\beta_{2}$-adrenergic receptor stimulation that directly signaled to adenylyl cyclase, the pharmacological target of forskolin. ${ }^{25}$ We observed subject-specific residual CFTR function and VX-809-induced drug responses, upon forskolin or $\beta_{2}$-agonist stimulation [Fig. 5a to $c_{;}$see supplemental Fig. S7, a to $c$ for responses of the individual experiments and supplemental Fig. S7d for results with $\beta_{2}$-agonists other than salmeterol]. Subject-to-subject variation was more prominent for $\beta_{2}$-agonists compared to forskolin, indicating that $\beta_{2}$-agonist receptor signaling may be variable between subjects (Fig. 5c and supplemental Fig. S7d). We observed a strong correlation between forskolin-induced-uncorrected and VX-809corrected CFTR function ( $R=0.8449$, $P=0.0029$; Fig. 5d). The subject-specific responses were maintained throughout the period of rectal organoid culture for at least 6 months and after 4 months of storage in liquid nitrogen (Fig. 5e to $h$ and supplemental Fig. S7e to $h$ ).

Together, these data indicated that subject-specific residual CFTR function and response to drug therapy could be detected independent of culture and technical variability in rectal organoids expressing identical CF-causing mutations. This demonstrated the impact of the subject-specific genetic background on the modulation of residual CFTR function and response to drug therapy.

\section{Organoid-based selection of clinical responders to VX-770}

Two subjects with the rare CFTR genotype G1249R/F508del were selected for in vivo treatment with ivacaftor on the basis of their effective response to $\mathrm{VX}-770$ in rectal organoids derived from these subjects (Figs. 3C and 6A and supplemental Fig. S4a). The G1249R mutation was described previously as a mutation in exon 20 of the CFTR gene, but the functional impact of this mutation has not been described. ${ }^{26}$ Upon treatment of the subjects carrying the G1249R mutation with VX-770 (150 mg twice daily) for 4 weeks, the CFTR-dependent marker (i) NPD (Fig. 6b to d) and (ii) SCC (Fig. 6b,e) improved or became normalized. Both subjects showed improved pulmonary function as measured by a decrease in airway resistance (RAW0.5) (Fig. 5f). FEV1 increased by $13 \%$ in subject 2 , whereas no clear response in FEV1 was observed in subject 1, who had a lower baseline $\mathrm{FEV}_{1}$ (Fig. 6g). Both patients improved with an increase in body weight and better scores on the respiratory domain of the CF-related quality of life questionnaire (Fig. 6b). After a washout period of 4 weeks, airway parameters decreased and CFTR-dependent biomarkers returned to pretreatment levels (Fig. 6, b and $d$ to g). Another subject with the F508del/ R347P mutation whose rectal organoids displayed a relatively weak response to VX-770 (Fig. 3c and supplemental Fig. S4a) showed a limited response to VX-770 in vivo, with a SCC of $-14 \mathrm{mmol} / \mathrm{L}$ and no improvement in $\mathrm{FEV}_{1}(-1 \%)$ after 4 weeks of treatment (Supplemental 
Fig. S8). This suggested that the R347P mutation is not responsive to VX770 treatment. ${ }^{27}$ These data indicate that in vitro rectal organoid assays may be able to identify clinical responders to VX-770 even when they express extremely rare uncharacterized CFTR mutations.

\section{DISCUSSION}

Here, we have studied subject-specific residual CFTR function and response to CFTRmodulating drugs in rectal organoids from CF patients. The data are most consistent with a model in which residual function and response to therapy are continuous variables (Fig. 2d and Fig. 3e), dependent on both the CF-causing mutation and additional subjectspecific genetic modifiers. Our data indicate that the current classification model that associates residual CFTR function with only class IV and V mutations may be inaccurate. We suggest that a refinement is needed that more accurately types residual function and pharmacological responses for different CFTR mutations and integrates subjectspecific genetic modifiers to indicate the breadth of functional variability for a particular CFTR genotype.

Both the FIS assay and the newly developed SLA assay have specific characteristics. The FIS assay is more sensitive than the SLA assay, and this simple, rapid swelling readout is optimally suited to compare CFTR residual function in CFTR mutant rectal organoids (Fig. 3a and supplemental Fig. S4a). However, differences in SLA between healthy control and CF rectal organoids prevented direct comparison of these groups (Fig. 1 and supplemental Fig. S1). The SLA assay has a dynamic window with higher CFTR function and allows for comparisons of drug responses between healthy control and CF organoids (Fig. 4b). However, it is less well suited for measuring residual CFTR function in organoids containing CF-causing mutations (Fig. 1 and supplemental Fig. S1). Potentially, the SLA readout may also be used to quantitate CFTR function for mutations associated with milder CFTR-related diseases. The SLA assay correlated with measures of in vivo efficacy upon overnight treatment with drug (Fig. 4d and supplemental Fig. S5) but required a time-consuming quantification method with the current set-up and software (Fig. 1b). The SLA and FIS assays together should help to type residual CFTR function and drugmodulated CFTR function for many CFTR mutations.

The relationship between the CFTR genotype and FIS (Fig. 2b,c) reflects published CFTR genotype-phenotype relationships obtained from clinical registries (www.CFTR2.org). ${ }^{28-30}$ Mutations 1811+1G>C, 3120G>A, 117-1G>T, N1303K, R347P and F508del were associated with severe CF and low FIS. Mutations A455E, S1251 N and R334W were associated with milder CF and moderate FIS. Mutations R117H-7T and TG(13)T(5) were associated with CFTR-related diseases and high FIS. Correlations among in vitro organoid responses 
and established CFTR-dependent markers (SCC and ICM) at the individual level (Fig. 2e,f) further supported our hypothesis that individual residual function measurements in organoids from subjects with CF may have complementary value to current approaches as a diagnostic or prognostic marker for individual patients. The many measurements from organoids compared to SCC measurements in vivo and ICM in ex vivo rectal biopsies allow for better control of technical variability and the establishment of forskolin dose ranges that precisely type residual CFTR function. The repeated measurements with organoids and strict CFTR dependency, as supported by analysis of organoids with CFTR null alleles or treated with CFTR inhibitors, in this study and other previous studies, may better indicate subjects who differ in residual CFTR function. ${ }^{12-14}$ To further optimize the detection of small differences in CFTR function among organoids, technical variation may be further minimized by including identical reference organoids on experimental plates. Direct studies that compare FIS levels or other individual markers are needed to compare their value for prediction of individual CF disease severity.

We observed clear differences between residual function and response to therapy among organoids with identical CF-causing mutations that were stable over extended culture periods (28 weeks), different media batches, and independent of biobank sources (Figs. $2 d$ and $3 e$ and $f$, and supplemental Fig. S7). These data extend previous observations demonstrating long-term genomic integrity and epigenetic profile stability of organoid cultures, and point to the individual genetic background as a modifier of CFTR function and fluid transport. ${ }^{9,31-33}$ Given that organoid swelling is fully CFTR-dependent, variation in swelling observed among organoids derived from different subjects most likely relates to the amount of active apical CFTR channels. ${ }^{12-14}$ This may depend on genetic variability (i) in the CFTR gene itself (as only the CF-causing mutations were characterized) or (ii) in other genomic regions that modify CFTR transcription, translation, post-translational processing (for example, folding efficacy and protein stability at the plasma membrane), or signaling efficacy between forskolin and CFTR. In addition, non-CFTR factors that modify the driving force for ion transport through CFTR may contribute to subjectto-subject variability, such as (i) function of basolateral and apical ion transporters or channels, (ii) aquaporin expression, or (iii) paracellular fluid transport. ${ }^{14}$ Subject-specific drug efficacy may further be modulated by genetic variability in drug efflux pumps. These potential mechanisms need to be addressed in more detail in future studies, as well as the relevance of variation in swelling in organoids from subjects with identical CF-causing mutations for clinical outcome.

Our data suggest that CFTR function analysis in rectal organoids may be used to select individuals for treatment with CFTR modulators (Figs. 3 and 5). The established forskolin dose ranges were critical to determine the optimal assay conditions for in vivo correlations. We found the highest positive correlation with clinical responses at a suboptimal forskolin dose $(0.128 \mu \mathrm{M}$; Fig. 3d). Defining a single forskolin concentration will be particularly helpful when analyzing larger patient populations in follow-up studies 
to define in vitro-in vivo relationships by limiting the amount of data points. At a forskolin concentration of $0.128 \mu \mathrm{M}$, the dynamic range of the FIS assay after drug treatment did not yet reach its maximum for organoids expressing mutations with high residual function such as R117H-7T (Fig 3A, supplemental Fig. S4), whereas drug efficacy for CFTR variants with low residual function could be detected. Furthermore, average drug responses of F508del/F508del mutant organoids were about twofold higher than those of F508del/class I mutant organoids (Fig. 3c), suggesting that the assay readout was linear. Whereas prediction of VX-809 and VX-770 drug efficacy in rectal organoids may be optimal at $0.128 \mu \mathrm{M}$ forskolin, other forskolin doses may be better for establishing correlations with other treatments or clinical parameters (for example the relationship between SCC and residual CFTR function was optimal at $0.8 \mu \mathrm{M}$ ).

Comparing responses between monotherapy and combination drug treatments requires a note of caution. It is likely that differences between in vivo pharmacokinetics of VX-770 and VX-770 + VX-809 are not fully reflected in vitro. Two drugs may not optimally reach the target tissue in vivo in the way that one drug does. This can result in discrepancies between in vitro and in vivo effects when different treatment modalities are compared. For instance, the responses of A455E organoids to combination treatment and S1251N organoids to VX-770 monotherapy are comparable, but similar effects may not be observed in vivo (Fig. 3c). On the other hand, we do expect that subjects with A455E will show a better response to the combination therapy in vivo than patients with two F508del mutations, as suggested by our in vitro results (Fig. 3c). Thus, analyses of drug responses in rectal organoids and different treatment modalities in vivo may provide insight into differences between pharmacokinetic properties of different treatments.

Proof of principle for preselecting subjects with uncharacterized CFTR mutations for drug therapy using rectal organoid-based assays was demonstrated upon treatment of two subjects with F508del/G1249R mutations with VX-770 (ivacaftor / KALYDECO) (Fig. 6). The subject with a milder baseline pulmonary phenotype showed a clear improvement in FEV1 after treatment. The lack of improvement of FEV1 in the other subject likely resulted from structural damage associated with severe pulmonary disease and fibrosis that may have prevent a FEV1 response to drug therapy. ${ }^{34}$ The improvement in RAW0.5 was consistent with the curvilinear relation between FEV1 and RAW0.5 (that is, a higher responsiveness of RAW0.5 at low FEV1). ${ }^{35,36}$ Changes in RAW0.5 in the G1249R-carrying subjects appeared relevant because short-term repeatability studies of this marker that indicated variability under identical conditions in patients with stable wheezing disorder $( \pm 28 \%)$ or healthy controls $( \pm 20 \%)$ were smaller. ${ }^{37}$ Positive treatment effects were achieved for other parameters, including two CFTR-dependent markers (SCC and NPD) (Fig. 6, $B$ to $E$ ) that supported the in vivo efficacy of VX-770. Notably, the in vivo treatments for the three subjects in this study were not blinded, which could have introduced an observation bias. In this context, the strong and consistent improvements in objective CFTR markers (SCC and NPD) were the strongest indications of an in vivo response. For 
Figure 6. Rectal organoid-based selection of clinical responders to VX-770 treatment.

a.

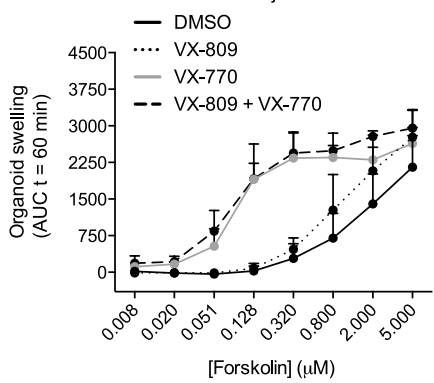

\section{F508del / G1249R}

Subject 2

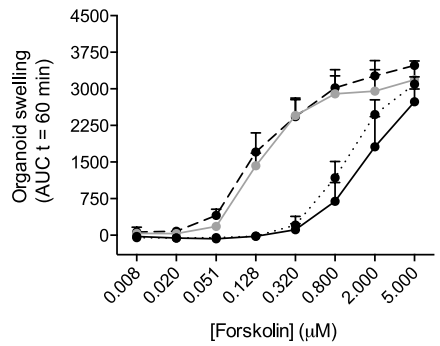

b.

\begin{tabular}{|c|c|c|c|c|c|c|}
\hline & \multicolumn{3}{|c|}{ Subject 1} & \multicolumn{3}{|c|}{ Subject 2} \\
\hline Sex & \multicolumn{3}{|c|}{ Male } & \multicolumn{3}{|c|}{ Female } \\
\hline Age & \multicolumn{3}{|c|}{17} & \multicolumn{3}{|c|}{39} \\
\hline & Baseline & $\begin{array}{l}4 \text { weeks } \\
\text { Ivacaftor }\end{array}$ & $\begin{array}{l}4 \text { week } \\
\text { washout }\end{array}$ & Baseline & $\begin{array}{l}4 \text { weeks } \\
\text { Ivacaftor }\end{array}$ & $\begin{array}{l}4 \text { week } \\
\text { washout }\end{array}$ \\
\hline $\begin{array}{l}\text { Weight } \\
(\mathrm{Kg})\end{array}$ & 57.0 & 58.7 & 59.8 & 66.5 & 67.2 & 67.5 \\
\hline $\begin{array}{l}\mathrm{FEV}_{1} \\
\text { (\% predicted) }\end{array}$ & 32 & 32 & 31 & 52 & 65 & 59 \\
\hline $\begin{array}{l}\text { RAW } 0.5 \\
\text { (\% predicted) }\end{array}$ & 354 & 266 & 401 & 144 & 95 & 125 \\
\hline $\begin{array}{l}\text { Sweat } \\
\text { chloride } \\
\text { (mmol/L) }\end{array}$ & 78 & 14 & 62 & 89 & 57 & 91 \\
\hline $\begin{array}{l}\text { NPD response } \\
\text { to Cl-free + iso } \\
(\mathrm{mV})\end{array}$ & -1 & -40 & 13 & -2 & -56 & -6 \\
\hline $\begin{array}{l}\text { CFQ-r } \\
\text { Respiratory } \\
\text { domain }\end{array}$ & 67 & 89 & 89 & 28 & 100 & 78 \\
\hline
\end{tabular}

(a) F508del/G1249R mutant rectal organoids derived from two different donors were treated with DMSO (vehicle), VX-809 (3 $\mu \mathrm{M}), V X-770(3 \mu \mathrm{M})$, or VX-809 and VX-770 combined and were stimulated with forskolin. (The average response of both subjects is presented in supplemental Fig. S4a.) Subjects were measured at 5 (subject 1) or 4 (subject 2) independent culture time points in duplicate. Data are means $\pm \mathrm{SD}$.

(b) Baseline characteristics and VX-770 (ivacaftor / KALYDECO) treatment for two CF subjects expressing the F508del/G1249R mutation. RAW0.5, airway resistance at a flow rate of 0.5 liters/s; Cl-free, chloride-free; Iso, isoproterenol.

(c) Tracings of NPD measurements before and after treatment of subject 1 $\neg$, performed according to the SOP of the European Cystic Fibrosis Society Clinical Trial Network (ECFS-CTN). Measurements in the right and left nostril were comparable. Am, Ameloride; ATP, adenosine 5'-triphosphate.

(d to g) Effects of 4-week VX-770 treatment (KALY) and a 4-week washout period (W) for NPD (d), SCC (e), RAWO.5 (f) and FEV1 (g). 
c.
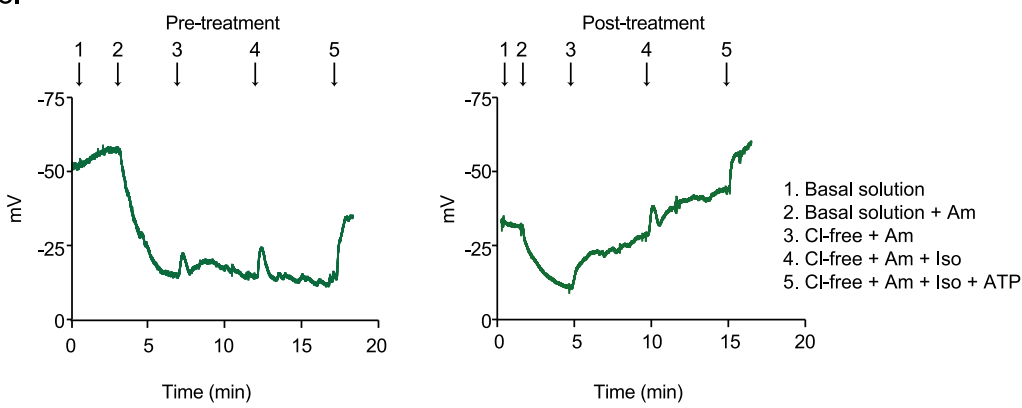

d.

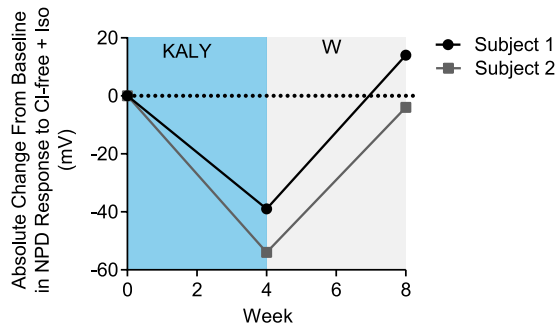

e.

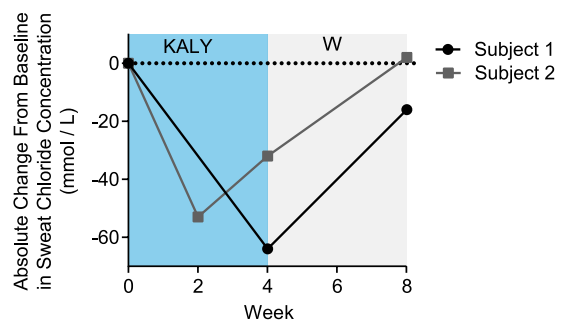

f.

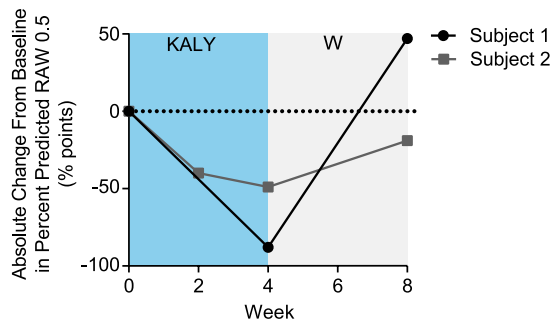

g.

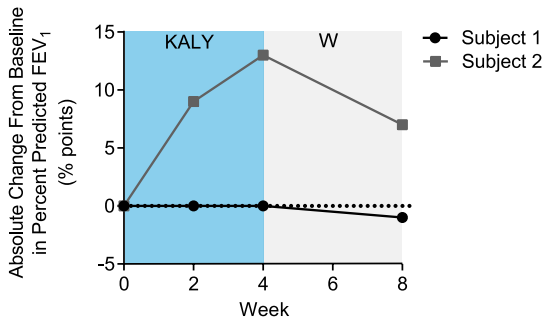

now, these anecdotal data suggest that a relatively simple preclinical test may help in clinical decision-making concerning the application of CFTR modulator therapy in CF patients with rare CFTR mutations.

Thus far, we have been able to recapitulate most observations about responses to CFTR modulators such as VX-809 and VX-770 in primary airway cell cultures. ${ }^{27,38}$ Recent data showed that chronic VX-770 treatment diminished VX-809-restored CFTR-F508del 
expression and function in primary airway cells. ${ }^{39,40}$ However, we observed with our SLA assay that chronic VX-770 and VX-809 cotreatment was better at restoring CFTRF508del function than treatment with a single drug (Fig. 4). Differences in experimental conditions between CFTR function measurements in airway cells and rectal organoids may likely contribute to these observations. The SLA assay in rectal organoids is a cumulative steady-state readout without a supraphysiological stimulus that cannot be compared directly with chronic VX-770 stimulation followed by an acute CFTR function measurement in airway cells. ${ }^{39,40} \mathrm{An}$ increase in CFTR gating and a decrease in CFTR expression by VX-770 treatment could have resulted in a net increase in SLA over the course of the experiment ( 24 or 48 hours) (Fig. 4). In addition, tissue specificity may also have an impact on the observed differences. For instance, a higher cell turnover rate in rectal organoids compared to differentiated epithelial airway cultures may affect the kinetics of apical CFTR delivery and removal. The downmodulation of VX-809-corrected F508del by chronic VX-770 treatment may be replaced more quickly by newly synthesized CFTR in rectal organoids compared to airway cells and may alter interactions with drugs. Both cell models are relevant and complementary for preclinical drug development and should enable further study of the potential tissue-specific effects of CFTR-targeting compounds.

Several limitations of this study need to be highlighted. Only three subjects were selected for ivacaftor treatment, and larger prospective in vitro-in vivo correlation studies for different CFTR modulators are required to further define the relationship between the preclinical rectal organoid readout and in vivo clinical drug responses. Double-blinded n-of-1 clinical trials with placebo and active drug treatment cycles will help to objectively measure clinical efficacy in individual settings and may help to interpret changes in clinical end points with considerable intrasubject variability such as $\mathrm{FEV}_{1}$ or RAW R.5 $_{\text {(Fig. }}$. 6). ${ }^{41}$ Longitudinal follow-up is needed to define robust sets of individual markers that are associated with long-term clinical efficacy of CFTR modulators. Combined in vitro and in vivo measurements in double-blind short-term treatment settings will possibly be more informative.

In addition, the 3D organization of rectal organoids that enables the swelling readout also results in limitations. Rectal organoids may rupture upon induction of swelling, although we have only rarely observed this in human organoids over the 60 -min time frame of the assay; we have never observed rupture of CF human organoids. Whereas organoid swelling at low swell rates is fully linear over a 60 minute time frame, a small curvilinear relation is observed beyond $\sim 30$ minutes for high swell rates (for example, with the $\mathrm{R} 117 \mathrm{H}$ mutation), leading to underestimation of CFTR function. Potentially, CFTR function itself may be regulated during cell stretching, although the linear swell response suggests that such an effect does not occur. ${ }^{42}$ In contrast to electrophysiological measurements with Ussing chambers in 2D systems, selective delivery of compounds to the apical or basolateral compartment in organoids is complicated. Apical stimulation 
can be performed in organoids by microinjection, but this is especially challenging for CF organoids due to their limited luminal volume. ${ }^{43}$ Recently described protocols to generate $2 \mathrm{D}$ monolayers from 3D organoids in culture may help in the study of ion channel activities that are not coupled to fluid secretion. ${ }^{44}$ This would allow for direct comparison of swelling phenotypes and transepithelial current measurements.

\section{CONCLUSION}

In conclusion, here, we have established a relationship between the CFTR genotype, residual CFTR function, and response to therapy using rectal organoids from 71 subjects with CF. We provide a proof of principle that individual in vitro functional measurements in rectal organoids may be used to preclinically select those subjects with CF who will respond to CFTR-modulating drugs. Once rectal organoids are established, they can be biobanked for testing of future drugs and therapeutic combinations in a cost-effective manner. Our data indicate that organoid-based CFTR function measurements can play an important role in the study of rare CFTR mutations and may help to identify subjects with CF who may benefit from CFTR modulator therapy independent of the CFTR mutation.

\section{ACKNOWLEDGEMENTS}

We thank S. Heida-Michel, M. Geerdink, M.C.J. Olling-de Kok (Department of Pediatric Pulmonology, Wilhelmina Children's Hospital, University Medical Center, Utrecht, Netherlands), E.M. Nieuwhof-Stoppelenburg, E.C. van der Wiel (Department of Pediatric Pulmonology, Erasmus University Medical Center/Sophia Children's Hospital, Rotterdam, Netherlands), J. Hulst, F.T.M. Kokke, B.A.E. de Koning (Department Pediatric Gastroenterology, Erasmus University Medical Center/Sophia Children's Hospital, Rotterdam, Netherlands), the technicians of the Clinical Chemistry Department (Erasmus University Medical Center/Sophia Children's Hospital, Rotterdam, Netherlands), N. Adriaens (Department of Respiratory Medicine, Academic Medical Center, Amsterdam, Netherlands), and M. Smink (Department of Pulmonology \& Cystic Fibrosis, Haga Teaching Hospital, The Hague, Netherlands) for providing intestinal biopsies and A.G.M Bot, M. Rampersad (Department Clinical Chemistry department, Erasmus University Medical Center/Sophia Children's Hospital, Rotterdam, Netherlands) and M.J.C. Bijvelds (Department of Gastroenterology and Hepatology, Erasmus University Medical Centrer/ Sophia Children's Hospital, Rotterdam, Netherlands) for performing ICM.

Funding: This work was supported by grants of the Dutch Cystic Fibrosis Foundation (NCFS) as part of the HIT-CF program and the Dutch Health Organization ZonMw, Netherlands. 


\section{COMPETING INTERESTS}

J.M.B. received travel funds and speaker honaria from Vertex Pharmaceuticals, Novartis, and Pfizer. J.M.B., C.K.E., J.F.D., and H.C. are inventors on a patent application related to these findings: "A rapid quantitative assay to measure CFTR function in a primary intestinal culture mode" (\#WO 2013093812 A2). H.C. is an inventor on other patents related to these findings: "Culture medium for epithelial stem cells and organoids comprising said stem cells" (\#WO 2010090513 A2. 2) and "Improved culture method for organoids" (WO 2015173425 A1). 


\section{REFERENCES}

1. Bell SC, De Boeck K, Amaral MD. New pharmacological approaches for cystic fibrosis: Promises, progress, pitfalls. Pharmacol Ther. 2015;145:19-34.

2. Sosnay PR, Siklosi KR, Van Goor F, et al. Defining the disease liability of variants in the cystic fibrosis transmembrane conductance regulator gene. Nat Genet. 2013;45(10):11601167.

3. Cutting GR. Cystic fibrosis genetics: from molecular understanding to clinical application. Nat Rev Genet. 2014;(November).

4. $\quad$ Ramsey BW, Davies J, McElvaney NG, et al. A CFTR potentiator in patients with cystic fibrosis and the G551D mutation. N Engl J Med. 2011;365(18):1663-1672.

5. Accurso FJ, Rowe SM, Clancy JP, et al. Effect of VX-770 in persons with cystic fibrosis and the G551D-CFTR mutation. N Engl J Med. 2010;363(21):1991-2003.

6. De Boeck K, Munck A, Walker $S$, et al. Efficacy and safety of ivacaftor in patients with cystic fibrosis and a non-G551D gating mutation. J Cyst Fibros. 2014;13(6):674-680.

7. Boyle MP, Bell SC, Konstan MW, et al. A CFTR corrector (lumacaftor) and a CFTR potentiator (ivacaftor) for treatment of patients with cystic fibrosis who have a phe508del CFTR mutation: a phase 2 randomised controlled trial. Lancet Respir Med. 2014;2(7):527-538.

8. Wainwright CE, Elborn JS, Ramsey BW, et al. Lumacaftor-Ivacaftor in Patients with Cystic Fibrosis Homozygous for Phe508del CFTR. N Engl J Med. 2015:150517100015004.

9. Sato $T$, Stange DE, Ferrante $M$, et al. Long-term Expansion of Epithelial Organoids From Human Colon, Adenoma, Adenocarcinoma, and Barrett's Epithelium. Gastroenterology. 2011;141(5):1762-1772.

10. Sato T, Clevers H. Growing Self-Organizing Mini-Guts from a Single Intestinal Stem Cell: Mechanism and Applications. Science (80- ). 2013;340(6137):1190-1194.

11. Dekkers JF, van der Ent CK, Beekman JM. Novel opportunities for CFTR-targeting drug development using organoids. Rare Dis. 2013;1(1):e27112.

12. Dekkers JF, Wiegerinck $\mathrm{CL}$, de Jonge $\mathrm{HR}$, et al. A functional CFTR assay using primary cystic fibrosis intestinal organoids. Nat Med. 2013;19(7):939-945.

13. Okiyoneda $\mathrm{T}$, Veit $\mathrm{G}$, Dekkers JF, et al. Mechanism-based corrector combination restores $\triangle F 508-C F T R$ folding and function. Nat Chem Biol. 2013;9(7):444-454.

14. Foulke-Abel J, In J, Yin J, et al. Human Enteroids as a Model of Upper Small Intestinal Ion Transport Physiology and Pathophysiology. Gastroenterology. 2016;150(3):638-649.e8.

15. Derichs N, Sanz J, Von Kanel T, et al. Intestinal current measurement for diagnostic classification of patients with questionable cystic fibrosis: validation and reference data. Thorax. 2010;65(7):594-599.

16. Sousa M, Servidoni MF, Vinagre AM, et al. Measurements of CFTR-Mediated Cl- Secretion in Human Rectal Biopsies Constitute a Robust Biomarker for Cystic Fibrosis Diagnosis and Prognosis. Hartl D, ed. PLoS One. 2012;7(10):e47708.

17. de Jonge HR, Ballmann $M$, Veeze $H$, et al. Ex vivo CF diagnosis by intestinal current measurements (ICM) in small aperture, circulating Ussing chambers. J Cyst Fibros. 2004;3:159-163.

18. De Boeck K, Derichs N, Fajac I, et al. New clinical diagnostic procedures for cystic fibrosis in Europe. J Cyst Fibros. 2011;10 Suppl 2:S53-66.

19. Mall M, Hirtz S, Gonska T, Kunzelmann K. Assessment of CFTR function in rectal biopsies for the diagnosis of cystic fibrosis. J Cyst Fibros. 2004;3:165-169.

20. Li H, Sheppard DN, Hug MJ. Transepithelial electrical measurements with the Ussing chamber. J Cyst Fibros. 2004;3:123-126.

21. Gibson LE, Cooke RE. Test for the concentration of electrolytes in cystic fibrosis of the pancreas utilizing pilocarpine by iontophoresis. Pediatrics. 1959;23:545-549. 
22. Quinton PM. Chloride impermeability in cystic fibrosis. Nature. 1983;301(5899):421-422.

23. Flume PA, Liou TG, Borowitz DS, et al. Ivacaftor in Subjects With Cystic Fibrosis Who Are Homozygous for the F508del-CFTR Mutation. Chest. 2012;142(3):718-724.

24. Clancy JP, Rowe SM, Accurso FJ, et al. Results of a phase lla study of VX-809, an investigational CFTR corrector compound, in subjects with cystic fibrosis homozygous for the F508del-CFTR mutation. Thorax. 2012;67(1):12-18.

25. Uezono Y, Bradley J, Min C, et al. Receptors that couple to 2 classes of G proteins increase CAMP and activate CFTR expressed in Xenopus oocytes. Receptors Channels. 1993;1(3):233-241.

26. Dijkstra DJ, Scheffer H, Buys CH. A novel mutation (G1249R) in exon 20 of the CFTR gene. Hum Mutat. 1994;4(2):161-162.

27. Van Goor F, Yu H, Burton B, Hoffman BJ. Effect of ivacaftor on CFTR forms with missense mutations associated with defects in protein processing or function. J Cyst Fibros. 2014;13(1):29-36

28. Gan K-H, Veeze HJ, van den Ouweland A, et al. A Cystic Fibrosis Mutation Associated with Mild Lung Disease. N Engl J Med. 1995;333(2):95-99.

29. McKone EF, Emerson SS, Edwards KL, Aitken ML. Effect of genotype on phenotype and mortality in cystic fibrosis: a retrospective cohort study. Lancet. 2003;361(9370):16711676.

30. Antiñolo G, Borrego S, Gili M, Dapena J, Alfageme I, Reina F. Genotype-phenotype relationship in 12 patients carrying cystic fibrosis mutation R334W. J Med Genet. 1997;34(2):89-91.

31. Middendorp S, Schneeberger $\mathrm{K}$, Wiegerinck $\mathrm{CL}$, et al. Adult stem cells in the small intestine are intrinsically programmed with their location-specific function. Stem Cells. 2014;32(5):1083-1091.

32. Huch M, Gehart H, van Boxtel R, et al. Long-term culture of genome-stable bipotent stem cells from adult human liver. Cell. 2015;160(1-2):299-312.

33. Sato T, Vries RG, Snippert HJ, et al. Single Lgr5 stem cells build crypt-villus structures in vitro without a mesenchymal niche. Nature. 2009;459(7244):262-265.

34. Wood ME, Smith DJ, Reid DW, Masel PJ, France MW, Bell SC. Ivacaftor in severe cystic fibrosis lung disease and a G551D mutation. Respirol case reports. 2013;1(2):52-54.

35. Arets HGM, Brackel HJL, van der Ent CK. Applicability of interrupter resistance measurements using the MicroRint in daily practice. Respir Med. 2003;97(4):366-374.

36. Rocha A, Donadio MVF, Avila DV de, Hommerding PX, Marostica PJC. Using the interrupter technique to evaluate airway resistance in cystic fibrosis patients. J Bras Pneumol publicaça"o Of da Soc Bras Pneumol e Tisilogia. 38(2):188-193.

37. Sonnappa S, Bastardo CM, Wade A, Bush A, Stocks J, Aurora P. Repeatability and bronchodilator reversibility of lung function in young children. Eur Respir J. 2013;42(1):116124.

38. $\quad \mathrm{Yu} \mathrm{H}$, Burton B, Huang C-J, et al. Ivacaftor potentiation of multiple CFTR channels with gating mutations. J Cyst Fibros. 2012;11(3):237-245.

39. Cholon DM, Quinney NL, Fulcher ML, et al. Potentiator ivacaftor abrogates pharmacological correction of $\triangle F 508$ CFTR in cystic fibrosis. Sci Transl Med. 2014;6(246):246ra96.

40. Veit G, Avramescu RG, Perdomo D, et al. Some gating potentiators, including VX-770, diminish $\Delta$ F508-CFTR functional expression. Sci Transl Med. 2014;6(246):246ra97.

41. Lillie EO, Patay B, Diamant J, Issell B, Topol EJ, Schork NJ. The n-of-1 clinical trial: the ultimate strategy for individualizing medicine? Per Med. 2011;8(2):161-173.

42. Vitzthum C, Clauss WG, Fronius M. Mechanosensitive activation of CFTR by increased cell volume and hydrostatic pressure but not shear stress. Biochim Biophys Acta. 2015;1848(11 Pt A):2942-2951. 
43. de Lau W, Kujala P, Schneeberger K, et al. Peyer's patch M cells derived from Lgr5(+) stem cells require SpiB and are induced by RankL in cultured \&quot;miniguts\&quot;: Mol Cell Biol. 2012;32(18):3639-3647.

44. Moon C, VanDussen KL, Miyoshi H, Stappenbeck TS. Development of a primary mouse intestinal epithelial cell monolayer culture system to evaluate factors that modulate IgA transcytosis. Mucosal Immunol. 2014;7(4):818-828. 


\section{SUPPLEMENTALS}

Supplemental Figure S1. Characteristics of the SLA assay in organoids.

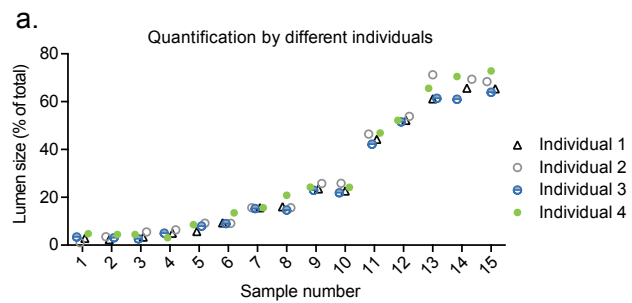

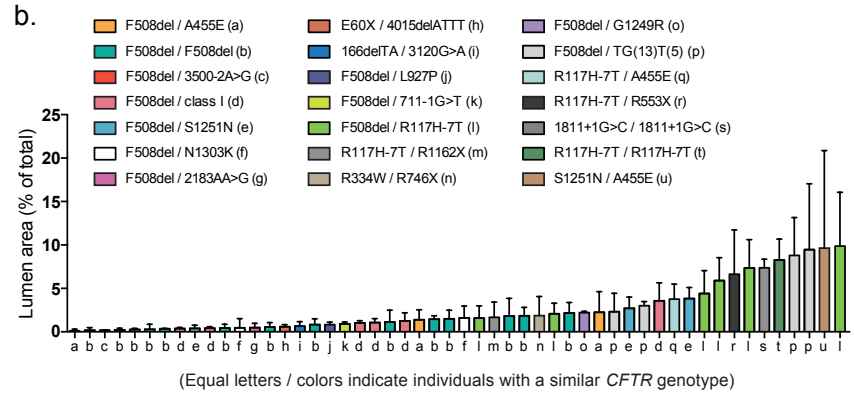
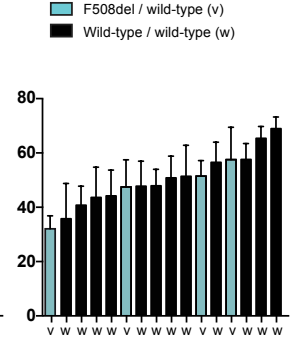

C.

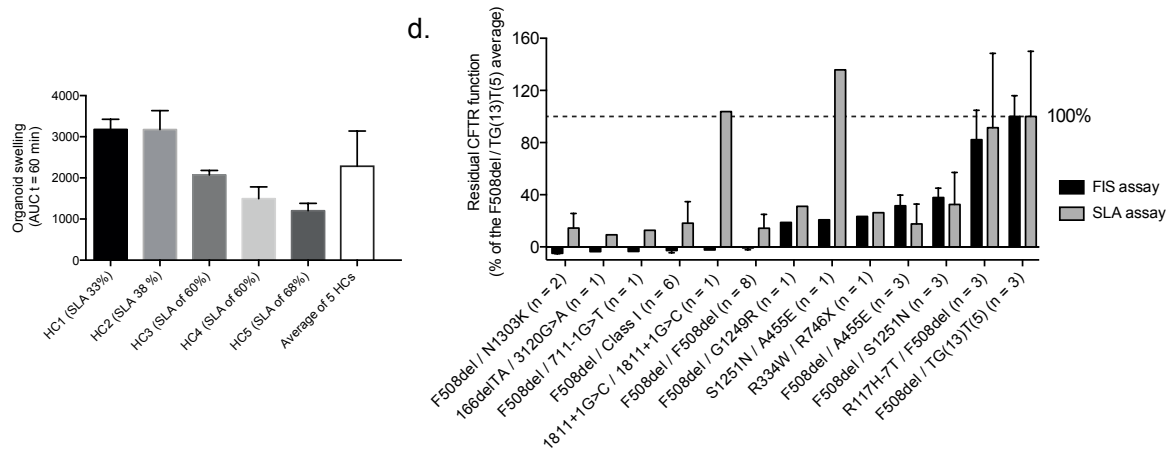

(a) Limited observer variability when steady-state organoid lumen area of different samples by four independent individuals was quantitated in a blinded fashion.

(b) Quantification of the SLA of organoids derived from individual donors (similar to Fig. 1c) expressing CFTR mutations as indicated. For each subject, 2 to 5 independent experiments were performed. Data are means \pm SD.

(c) FIS of HC organoids derived from 5 different donors with variable SLAs at the start of forskolin treatment ( $5 \mathrm{mM}$ ). SLA or FIS was calculated as explained in Fig. 1b or supplemental Fig. S3, respectively. Data was averaged from 2 to 3 different wells per donor. Data are means $\pm \mathrm{SD}$.

(d) Analysis of residual CFTR function in various mutant CFTR organoids measured by FIS (similar to FIS data presented in Fig. 2c) or the SLA. Responses are normalized to the average response of F508del / TG(13)T(5) organoids (100\%). Data are means \pm SD. (The class I mutations include G542X, R1162X (2 donors), W1282X, DELE2.3 and E60X). 
Supplemental Figure S2. Detectable residual CFTR function in several forskolin-stimulated organoids expressing severe CFTR mutations.

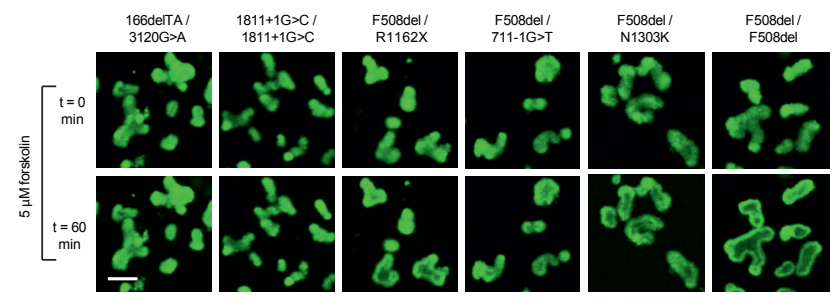

Representative confocal images of calcein-green-labeled organoids at the indicated time points of forskolin stimulation. Scale bar $80 \mu \mathrm{M}$. 
Supplemental Figure S3. Quantification of forskolin-induced organoid swelling.

a

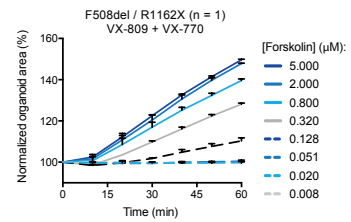

b.
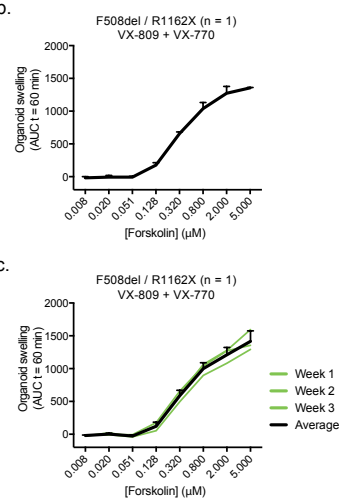

d.

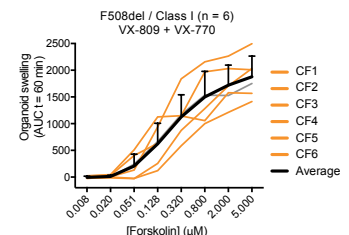

e.

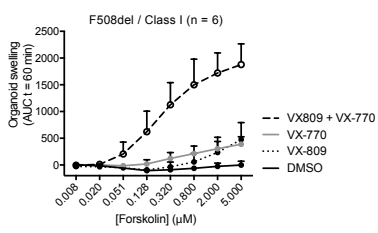

(a) Quantification of the surface area relative to $t=0$ (normalized organoid area) of drug-treated F508del/R1162X organoids at different forskolin concentrations averaged from two independent wells.

(b) FIS of organoids expressed as the absolute AUC calculated from a (baseline $=100 \%, t=60 \mathrm{~min}$ ).

(c) Quantification of FIS as described in (b) at three independent time points.

(d) FIS quantification as described in (c) of 6 different F508del/class I organoids.

(e) Averaged FIS of responses as shown in (d) for VX-809 (3 $\mu \mathrm{M})$ - and VX-770 (3 $\mu \mathrm{M})$-treated organoids. In addition, responses to DMSO, VX-809 or VX-770 are shown. Each data point represents responses of F508del/Class I organoids derived from six different individuals, measured at 2 to 3 independent culture time points in duplicate (Data are means \pm SD). 
Supplemental Figure S4. Genotype-specific profiles of residual and drug-corrected CFTR function in forskolin-stimulated mutant CFTR organoids.

a.
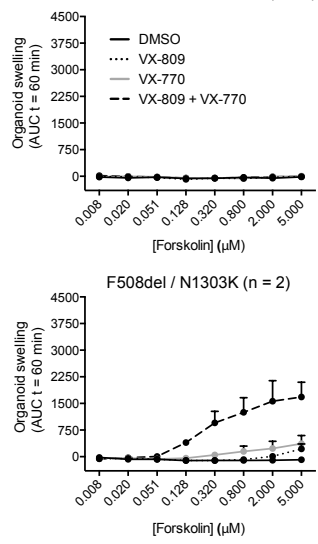

F508del / A455E $(n=3)$

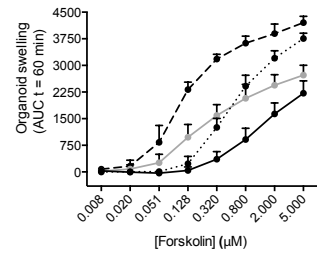

A455E / S1251N $(n=1)$

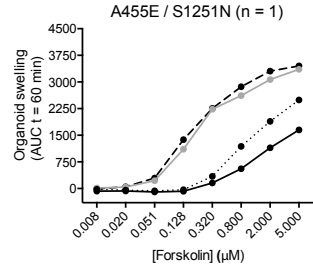

F508del / TG(13)T(5) $(n=3)$

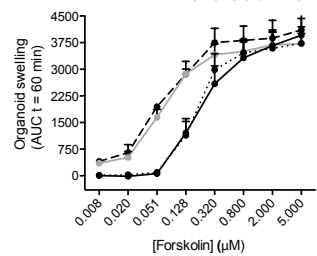

166deITA / 3120G $>A(n=1)$
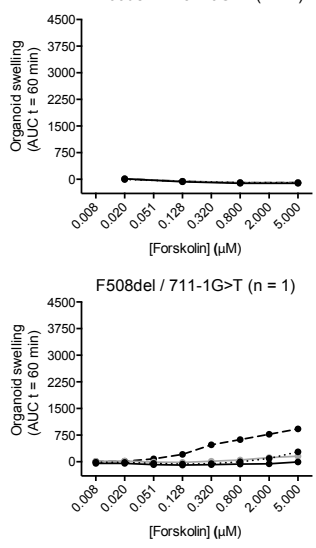

F508del / S1251N ( $=3)$

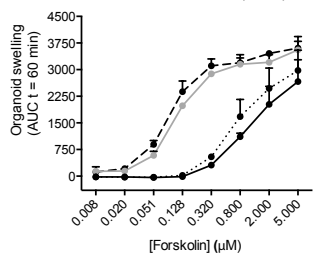

$\mathrm{R} 334 \mathrm{~W} / \mathrm{R} 746 \mathrm{X}(\mathrm{n}=1)$

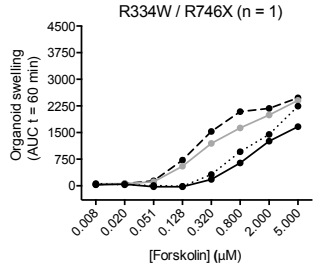

F508del / R347P $(n=1)$

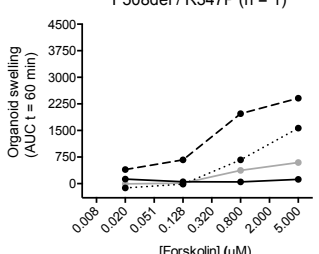

F508del / Class I $(n=6)$

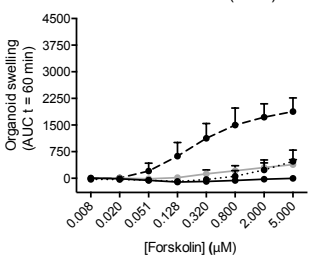

F508del / F508del $(n=9)$

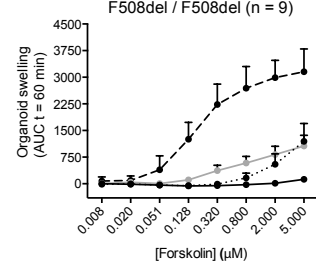

F508del / G1249R ( $n=2)$
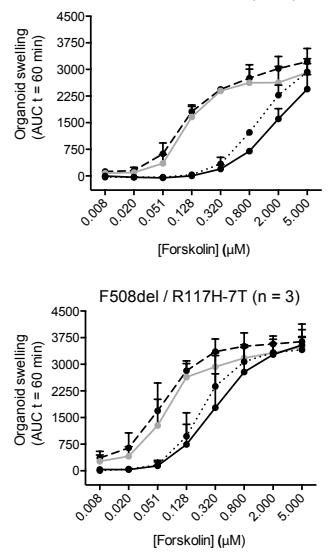

b.

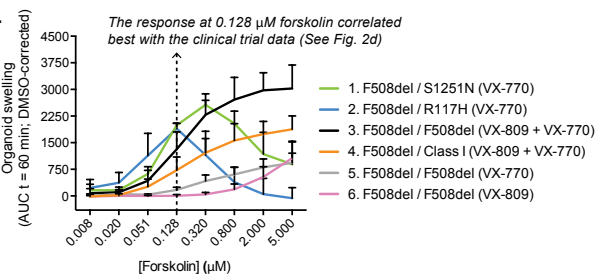

(a) FIS of various mutant CFTR organoids, which are untreated or treated with VX-809 (3 $\mu M), V X-770(3 \mu M)$ or both at the indicated forskolin concentrations. Swelling is presented as absolute AUC calculated from time tracings as shown in Fig. 2A and supplemental Fig. S3a (Baseline $=100 \%$; $=60 \mathrm{~min}$.) (Data are means \pm SD; data were generated during a period of 1.5 year, of which $~ 80 \%$ were generated during a period of 4 months using an identical medium batch to limit technical variation; $n=$ number of subjects; each subject was measured at 2 to 5 independent time points in duplicate: the class I mutations include G542X, R1162X (2 donors), W1282X, DELE2.3 and E60X).

(b) Responses of clinically assessed CFTR genotypes and treatments (see Fig. 3b) derived from (a), corrected for the DMSO-treated condition. 
Supplemental Figure S5. Data overview of clinical trials with CFTR-repairing treatment in subjects expressing different mutated CFTR genotypes.

a.

\begin{tabular}{|c|c|c|c|c|c|}
\hline Treatment & Phase & CFTR genotype & $\begin{array}{l}\text { Absolute change } \\
\% \text { predicted } \mathrm{FEV}_{1} \\
\text { versus placebo }\end{array}$ & $\begin{array}{l}\text { Relative change } \\
\% \text { predicted } \mathrm{FEV}_{1} \\
\text { versus placebo }\end{array}$ & Reference \\
\hline VX-770 & III & S1251N/other & $8.7 \%(p<0.0001)$ & No data & De Boeck et al, 2014 \\
\hline$V X-770$ & III & R117H-5T/7T / other & $5.0 \%(p=0.01)$ & $9.1 \%(p=0.008)$ & investors.vertex.com \\
\hline$V X-809+V X-770$ & III & F508del / F508del & $3.3 \%(p<0.0001)$ & $5.6 \%(p<0.001)$ & Wainwright et al, 2015 \\
\hline$V X-809+V X-770$ & II & F508del / other & $0.3 \%$ (NS) & $0.4 \%$ (NS) & Boyle et al, 2014 \\
\hline$V X-770$ & II & F508del / F508del & $1.7 \%(\mathrm{NS})$ & $2.4 \%$ (NS) & Flume et al, 2012 \\
\hline VX-809 & Ila & F508del / F508del & $0.27 \%(N S)$ & $0.4 \%$ (NS) & Clancy et al, 2011 \\
\hline
\end{tabular}

b.

$\begin{array}{ccc} & \begin{array}{c}\text { Absolute change } \\ \% \text { predicted FEV } \\ \text { versus placebo }\end{array} & \begin{array}{c}\text { Relative change } \\ \% \text { predicted FEV }\end{array} \\ \text { versus placebo }\end{array}$

(a) Per trial, results of the most optimal treatment strategy are presented. Of the R117H trial, only data from CF subjects aged $>18$ were used, as subjects aged 6 to 18 had a different mean baseline FEV1.

(b) Pearson correlation values of drug-repaired organoid data (see Fig. 3c for FIS data and Fig. 4c for SLA data) versus the lung function increase presented in (a). 
Supplemental Figure S6. Pearson correlations of residual CFTR function and response to therapy measured by the FIS assay.
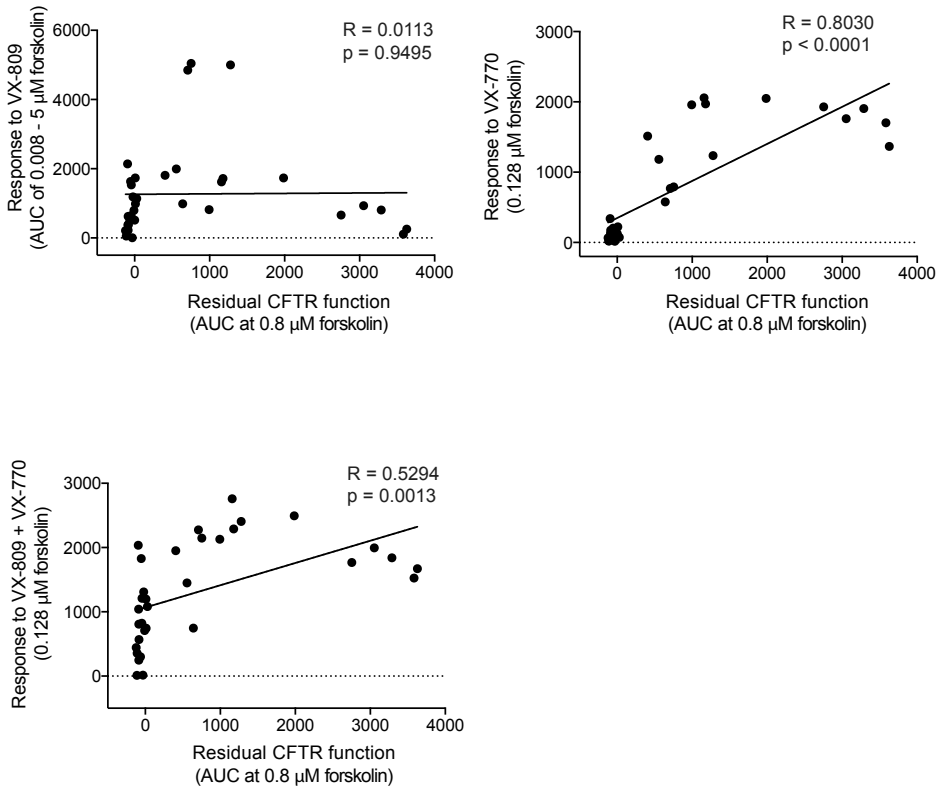

The residual function data (DMSO-treated FIS; AUC $\mathrm{t}=60$ min at $0.8 \mu \mathrm{M}$ forskolin; also see Fig. 1e-i and supplemental Fig. S4a) was correlated with the DMSO-corrected response to VX-809 (AUC of 0.008 - $5 \mu \mathrm{M}$ forskolin, baseline = 0; calculated from supplemental Fig. S4a). 
Supplemental Figure S7. CFTR correction in F508del homozygous organoids.
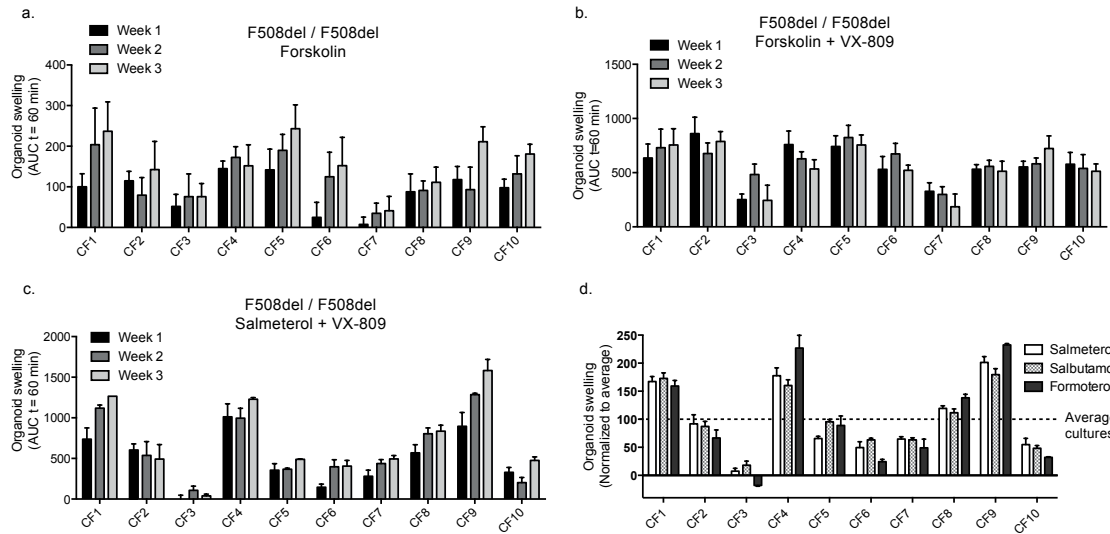

d.
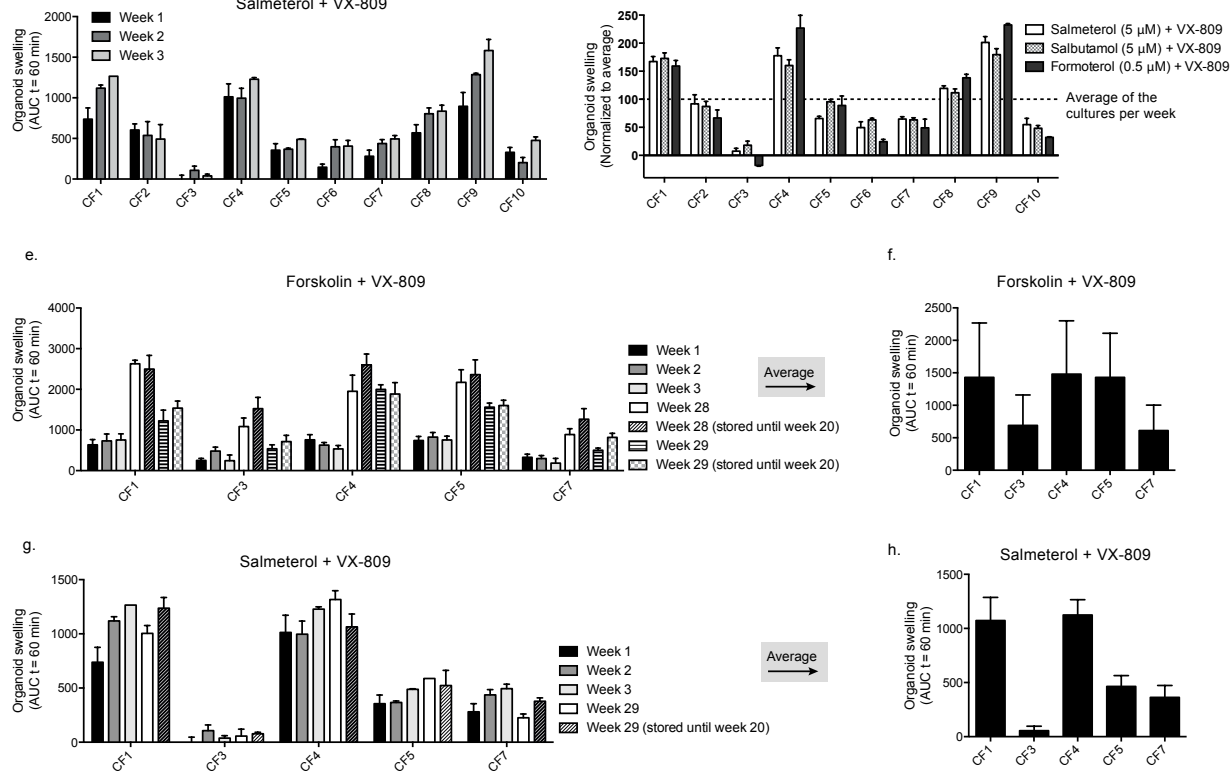

(a tot c) Swelling of organoids induced by forskolin (a) or pre-incubated for $24 \mathrm{~h}$ with VX-809 and induced by forskolin (b) or salmeterol (c), expressed as the absolute AUC calculated from time tracings comparable to Fig. 2A (baseline $=100 \%, t=60 \mathrm{~min}$ ). Responses were averaged from 3 independent wells. Data are means \pm SD. (see Fig. 5c for the averaged data)

(d) Swelling of the F508del homozygous organoid panel in response to salmeterol, salbutamol and formoterol, normalized to the average response of the 10 cultures.

(e to h) After the first 3 experiments (week 1 to 3; similar to data in (b) and (c), 2 low responding and 3 high forskolinresponding cultures were maintained in culture and measured again at week 28 and 29, or reinitiated after liquid nitrogen storage at week 20 and measured at week 28 and 29. Swelling of VX-809-corrected organoids in response to forskolin $(e, f)$ or salmeterol $(g, h)$ presented per experiment $(e, g)$ or as average $(f, h)$. See Fig. 5 e to $h$ for the normalized responses. In (e) and (g) the SD indicates the variation from 3 independent wells; in (f) and (h) the SD indicates variation between different experiments. Data are means \pm SD. 
Supplemental Figure S8. In vivo indications for organoid-based selection of a clinical non-responder to VX-770.

a.

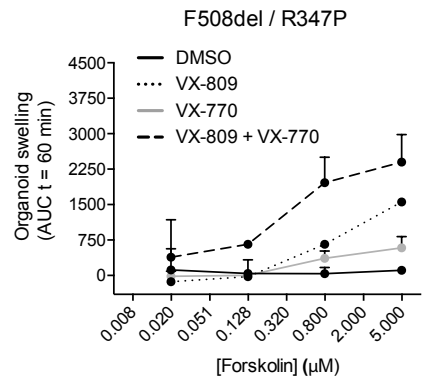

b.

\begin{tabular}{|c|c|c|c|}
\hline & \multicolumn{3}{|c|}{ Patient 1} \\
\hline Sex & \multicolumn{3}{|c|}{ Female } \\
\hline Age & \multicolumn{3}{|c|}{13} \\
\hline & Baseline & $\begin{array}{l}4 \text { weeks } \\
\text { Ivacaftor }\end{array}$ & $\begin{array}{l}4 \text { week } \\
\text { washout }\end{array}$ \\
\hline $\begin{array}{l}\text { Weight } \\
(\mathrm{Kg})\end{array}$ & 43.0 & 46.0 & 48.8 \\
\hline $\begin{array}{l}\mathrm{FEV}_{1} \\
\text { (\% predicted) }\end{array}$ & 95 & 94 & 87 \\
\hline $\begin{array}{l}\text { RAW } 0.5 \\
\text { (\% predicted ) }\end{array}$ & 113 & 140 & 277 \\
\hline $\begin{array}{l}\text { Sweat } \\
\text { chloride } \\
\text { (mmol/L) }\end{array}$ & 76 & 62 & 76 \\
\hline $\begin{array}{l}\text { CFQ-r } \\
\text { Respiratory } \\
\text { domain }\end{array}$ & 75 & 67 & 42 \\
\hline
\end{tabular}

C.

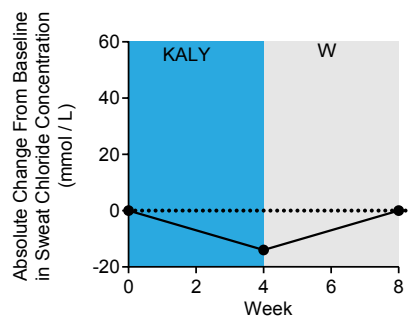

d.

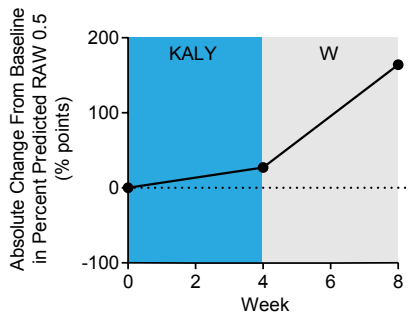

e.

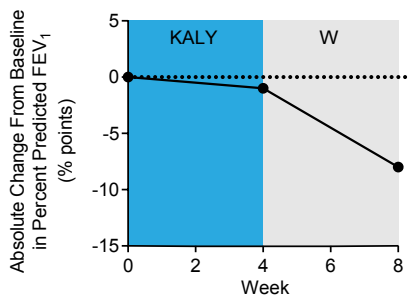

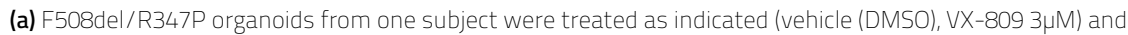
stimulated with forskolin. The same figure is presented in supplemental figure S4a. The subject was measured at three independent culture time points in duplicate and error bars indicate inter-experimental variation; Data are means $\pm S D$.

(b) Baseline characteristics and VX-770 (ivacaftor/KALYDECOTM) treatment effects of the subject expressing F508del/R347P.

(c to e) Effects of 4 weeks of VX770 (KALY) treatment and a 4-week washout (W) in SCC (c), RAW0.5 (d) and FEV1 (e). 
Supplemental Table S1. Overview of all CFTR genotypes and their corresponding mutation classes and gender.

\begin{tabular}{|c|c|c|}
\hline CFTR GENOTYPE & MUTATION CLASS & GENDER \\
\hline E60X / 4015delATTा & $|/|$ & Male \\
\hline 166delTA / 3120G>A & $1 /\left.\right|^{\mathrm{b}}$ & Male \\
\hline $1811+1 G>C / 1811+1 G>C$ & $|/|$ & Male \\
\hline F508del / DELE2.3 & $\| / 1$ & Male \\
\hline F508del / W1282X & $\| / 1$ & Male \\
\hline F508del / E60X & $\| / 1$ & Female \\
\hline F508del / G542X & $\| / 1$ & Female \\
\hline F508del / R1162X (2x) & $\| / 1$ & Male / Female \\
\hline F508del / 3500-2A>G & $\| /\left.\right|^{b}$ & Female \\
\hline F508del / 2183-AA>G & $\| / 1$ & Female \\
\hline F508del / N1303K (2x) & $\| /\left.\right|^{b}$ & Male / Female \\
\hline F508del / 711-1G>T & $\| / 1$ & Male \\
\hline F508del / R347P & $\| / 1$ & Female \\
\hline F508del / L927P & II / IV & Male \\
\hline F508del / F508del (15x) & $\|/\|$ & 8x Male / 7x Female \\
\hline R334W / R746X & IV /I & Male \\
\hline F508del / S1251N (3x) & $\|/\|$ & 1x Male / 2x Female \\
\hline F508del / G1249R (2x) & $\|/\|^{b}$ or $I V^{b}$ & 1x Male / 1x Female \\
\hline F508del / R117H-7T (6x) & II / IV & 2x Male / 4x Female \\
\hline $\mathrm{R} 117 \mathrm{H}-7 \mathrm{~T} / \mathrm{R} 553 \mathrm{X}$ & IV /I & Female \\
\hline R117H-7T / R1162X & IV /I & Female \\
\hline R117H-7T / R117H-7T & IV / IV & Female \\
\hline F508del / A455E (3x) & $\| / V$ and $\|^{b}$ & 2x Male / 1x Female \\
\hline S1251N / A455E & \|\|$/ V$ and $\|^{b}$ & Male \\
\hline R117H-7T / A455E & $\mathrm{IV} / \mathrm{V}$ and $\mathrm{I}^{\mathrm{b}}$ & Female \\
\hline F508del / TG(13)T(5) (4x) & $\| / V$ & 3x Male / 1x Female \\
\hline F508del / wild-type (4x) & $\| /-$ & 3x Male / 1x Female \\
\hline Wild-type / wild-type (12x) & $-1-$ & 8x Male / 4x Female \\
\hline
\end{tabular}

a Classification based on literature and/or the CFTR mutation database (www.genet.sickkids.on.ca),

${ }^{b}$ Classification based on functional CFTR measurements in organoids. 


\section{CHAPTER \\ 8}

\section{RECTAL ORGANOIDS ENABLE PERSONALIZED TREATMENT OF CYSTIC FIBROSIS}

Gitte Berkers, Peter van Mourik, Annelotte M. Vonk, Evelien Kruisselbrink, Johanna F. Dekkers, Karin M. de Winter - de Groot, Hubertus G.M. Arets, Rozemarijn E.P. Marck-van der Wilt, Jasper S. Dijkema, Maaike, M. Vanderschuren, Roderick H.J. Houwen, Harry G.M. Heijerman, Eduard A. van de Graaf, Sjoerd G. Elias, Christof J. Majoor, Gerard H. Koppelman, Jolt Roukema, Marleen Bakker, Hettie M. Janssens, Renske van der Meer12, Robert G.J. Vries, Hans C. Clevers, Hugo R. de Jonge, Jeffrey M. Beekman*, Cornelis K. van der Ent*.

* These authors contributed equally 


\section{ABSTRACT}

In vitro drug tests using patient-derived stem cell cultures offer opportunities to individually select efficacious treatments. Here, we provide a study that demonstrates that in vitro drug responses in rectal organoids from individual patients with cystic fibrosis correlate with changes in two in vivo therapeutic endpoints. We measured individual in vitro efficaciousness using a functional assay in rectum-derived organoids based on forskolin-induced swelling, and studied the correlation with in vivo effects. The in vitro organoid responses correlated with both change in pulmonary response and change in sweat chloride concentration. Receiver operating characteristic curves indicated good to excellent accuracy of the organoid-based test for defining clinical responses. This study indicates that an in vitro assay using stem cell cultures can prospectively select efficacious treatments for patients, and suggests that biobanked stem cell resources can be used to tailor individual treatments in a cost-effective and patient-friendly manner. 


\section{INTRODUCTION}

Functional drug testing on cells or tissue cultures of patients may represent a major step forward for selecting efficacious treatments in an individual setting. Our identification of Lgr5 as a marker of crypt stem cells and the development of technology to grow functional epithelial organoids from such stem cells allows the generation of disease- and patientspecific living biobanks. ${ }^{1-3}$ These biobanks could serve as important resources for drug development and scientific studies, but examples demonstrating the validity of these tissue resources for the individual prediction of clinical drug efficacy are currently lacking.

Cystic fibrosis (CF) is a genetic disease that is caused by mutations of the gene encoding for the cystic fibrosis transmembrane conductance regulator (CFTR) protein, which leads to impaired protein function. ${ }^{4}$

Over 2000 CFTR mutations have been identified (www.genet.sickkids.on.ca) and are associated with a variety of clinical phenotypes (www.cftr2.org) ${ }^{5,6}$ Recently developed drugs for CF aim to restore CFTR protein function. Lumacaftor (VX-809) and Tezacaftor (VX-661) are corrector drugs, influencing trafficking of the CFTR protein to the apical membrane, while ivacaftor (VX-770) is a potentiator drug, improving the function of the CFTR protein that is present at the apical membrane. In previous work we showed that also the natural food components genistein and curcumin have potentiator activity in vitro, albeit at reduced efficacy and potency as compared to ivacaftor. ${ }^{7}$ Currently three CFTR modulating drugs are registered for the treatment of CF patients with specific CFTR mutations; ivacaftor (VX770, Kalydeco ${ }^{\circledR}$ ) for patients with different CFTR gating mutations and patients with an $\mathrm{R} 117 \mathrm{H}$ mutation, and a combination of ivacaftor and the CFTR correctors lumacaftor or tezacaftor (resp VX770+VX809, Orkambi® and VX770+VX661, Symdeco/Symkevi®) for patients homozygous for the F508del mutation and some mutations associated with residual function in the case of Symdeco/Symkevi treatment. ${ }^{8-13}$

This CFTR genotype-based stratification for drug prescription presents a challenge for the inclusion of many people with rare CFTR mutations who are not included into clinical trials due to low prevalence of the mutation and lack of mechanistic insights. A recent label extension of ivacaftor by the US Food and Drug Administration (FDA) based on in vitro data of heterologous cell lines and mode-of-action, signals a paradigm shift of the regulatory pathway to faster drug access for people with rare CFTR mutations. ${ }^{14}$ In previous work we showed that forskolin induced swelling (FIS) of rectal organoids can be used to quantify the function of the CFTR protein in response to CFTR modulating drugs. Forskolin raises intracellular cyclic AMP that leads to opening of the CFTR ion channel and subsequent ion and fluid transport into the organoid lumen in a CFTR-dependent manner. This readout functionally assesses the impact of both CFTR mutations and additional patient-specific genetic factors that act on CFTR function. ${ }^{15}$ In previous work we showed that the in vitro response that was measured in rectal organoids correlates 
with average clinical responses described in patient populations with corresponding genotypes. ${ }^{16}$ We also predicted the lack of efficacy of PTC124 (ataluren) in a recent phase 3 clinical trial, by testing of PTC124 in rectal organoids from people carrying nonsense mutations. ${ }^{17,18}$ In vitro functional testing in rectal organoids of an individual patient may be a next step to facilitate rapid individual access to treatment for patients with rare CFTR mutations.

Currently it is not clear if the in vitro FIS response to CFTR modulating drugs correlates with the in vivo response at the level of the individual patient. Current clinical outcome parameters and in vivo or ex vivo biomarkers of CFTR function are highly valuable for measurement of average treatment effects in clinical trials, but they do not correlate at the individual level. A recent meta-study found a small correlation between the in vivo pulmonary response and the response of an in vivo biomarker of CFTR function (sweat chloride concentration (SCC)), but this study also indicated that individual responses in SCC had a low predictive value for corresponding pulmonary response. Our previous study with rectal organoids showed that two individuals who carried mutations that were not yet characterized, could be successfully selected for a treatment with ivacaftor. ${ }^{16}$ We also recently described that FIS measurements of individual patients were related to clinical indicators of CF disease severity, and comparison of FIS and SCC suggested more precise quantification of CFTR function by FIS. ${ }^{19}$ We here describe the correlation between the response of FIS of rectal organoids and the in vivo therapeutic response for individual CF patients with multiple CFTR genotypes who were treated with several CFTR modulating drugs, and we study the predictive values of the organoid FIS test for the clinical response.

\section{METHODS}

\section{FIS of rectal organoids}

Rectal organoids were cultured according to previously described protocols, and are accessible for study by contacting the Hubrecht Organoid Technology foundation (www.hub4organoids.eu). 16,20 FIS of rectal organoids is a fully CFTR-dependent readout and was measured to indicate baseline CFTR function and response to drugs. ${ }^{15,16}$ The organoid response to a drug was calculated by subtracting the dimethyl sulfoxide (DMSO) response at the same forskolin concentration.Organoid swelling was measured in duplicate at multiple independent culture time points as indicated in supplemental Fig. 51, with 4-8 different concentrations of forskolin as previously described. ${ }^{15,16,22}$ The CFTR modulators (3 $4 \mathrm{M}$ VX-770/ivacaftor (Selleck Chemicals LLC) or a combination of 10 $\mu \mathrm{M}$ genistein (Sigma) plus $50 \mu \mathrm{M}$ curcumin (Sigma)) were directly added to the organoids with forskolin, except for VX-809/lumacaftor (3 $\mu \mathrm{M}$, Selleck Chemicals LLC) that was pre-incubated for $24 \mathrm{~h}$. Organoids were fluorescently labeled and total area per well and time point was monitored by a Zeiss LSM800 confocal microscope. A Zen Image analysis 
software module (Zeiss) was used to quantify the organoid response (area under the curve measurements of relative size increase of organoids after 60 minutes forskolin stimulation, $\mathrm{t}=0$ min baseline of $100 \%$ ).

\section{Patient selection}

A total of 24 patients (15 males and 9 females, median age 16.0 years) were included in this study. From these 24 patients, 15 patients had at least one S1251N mutation and were treated with CFTR modulators as part of a clinical trial aiming to compare different CFTR potentiator treatments (NTR4585 and NTR4873). Thirteen of these 15 patients participated in both clinical trials and therefore received two different CFTR modifying treatments. The remaining 9 patients carried at least one rare CFTR mutation and were selected for off-label CFTR modulator treatments based on the organoid response and clinical necessity. A rare mutation was defined as a mutation with a prevalence of less than $1.0 \%$ in the Dutch CF population of which no data on clinical drug responsiveness was available in literature at the time of biopsy. ${ }^{21}$ More information on the clinical characteristics of the selected patients is shown in Table 1. All patients (and/or their legal representatives) gave informed consent for rectal biopsies, generating and testing of their individual organoids as well as for (data collection on the effect of) clinical treatment.

\section{Clinical endpoints}

In vivo therapeutic effect in the patients with an S1251N mutation was measured by absolute change after 8 weeks of CFTR modulator treatment in comparison with pretreatment baseline value. Data from people with rare mutations receiving either ivacaftor or lumacaftor/ivacaftor was collected between 4-8 weeks after initiation of treatment. Forced expiratory volume in one second is a widely used readout to assess pulmonary function, and was expressed as percent predicted for body height, age and gender (ppFEV1). SCC measurements were assessed as this is currently the best established in vivo biomarker of CFTR function

\section{Evaluation of clinical treatment}

For all treatments both the patients and those who were involved in clinical data collection were blinded for the magnitude of the in vitro drug response of the patients' organoids and vice versa. The ppFEV1 was measured according to ATS-ERS standards. ${ }^{23,24}$ The SCC was measured using the Macroduct ${ }^{\circledR}$ system and performed according to the most recent version of the standard operating procedure of the European Cystic Fibrosis Society-Clinical Trial Network. 


\section{Quantification and statistical analysis}

The primary outcome of the study was the correlation (Pearson) between the in vitro organoid and in vivo effects (change in $\mathrm{ppFEV}_{1}$ and SCC) plus the predictive capacity of the organoid model, in patients that had a baseline ppFEV1 between 40 and 90 percent. When a change in ppFEV 1 or SCC was missing, a patient was excluded from that part of the analysis. In a secondary analysis, we calculated the correlation and predictive capacity for patients that had a baseline ppFEV1 of $<40$ or $>90$ percent as well as for the total group of patients that was treated. Finally we used the wilcoxon signed rank test to examined the clinical response of patients with at least one rare CFTR mutation (nonF508del or $\mathrm{S} 1251 \mathrm{~N}$ ) who had a response in their rectal organoids (area under the curve (AUC) at $0.128 \mu \mathrm{M}$ forsklin $>1000$ ) to the CFTR modulating drug.

Receiver operating characteristic (ROC) curves were generated to evaluate the predictive capacity of organoid FIS for clinical responses. A Youden index was used to select the organoid cut-off point with the most optimal combination of sensitivity and specificity from the ROC-curves. ${ }^{25} \mathrm{~A}$ leave-one-out cross validation further validated our findings. ${ }^{26}$ As some patients were treated with two CFTR modifying treatments, we controlled for repeated measurements when calculating correlations and ROC-curves to evaluate a potential bias. ${ }^{27,28}$ Because of the limited number of patients, no further subgroup analysis were performed. Statistical analysis were performed using GraphPad Prism 7.02, IBM SPSS Statistics version 22 and R-studio version 0.99.441.

\section{Additional resources}

The clinical trial registry numbers and Institutional Review Board (IRB) numbers of the two trials in which the patients with an S1251N mutation were treated with genistein plus curcumin and ivacaftor are NTR4585/METC14-268/G-M and NTR4873/METC14$514 / \mathrm{M}$ respectively. Additional information on these trials can be found on http://www. trialregister.nl/trialreg/index.asp. The IRB code of the HUB-CF organoid biobank is 14008. 


\section{RESULTS}

To evaluate the relation between drug response in in vitro cultured organoids and therapeutic effect in vivo, we studied 37 paired in vitro-in vivo responses to three CFTR modulating treatments in 24 subjects with CF (baseline characteristics are provided in Table 1). Fifteen patients with the ivacaftor-responsive $\mathrm{S} 1251 \mathrm{~N}$ mutation received ivacaftor. ${ }^{9}$ Thirteen of these patients first received a combination of the possible CFTR potentiating food supplements genistein and curcumin before receiving ivacaftor. ${ }^{7}$ The other 9 patients carried at least one rare CFTR mutation with unknown clinical response and were selected for off-label treatment based on the organoid response to either ivacaftor or ivacaftor plus lumacaftor. Apart from the CFTR genotype there were no relevant differences in the baseline clinical characteristics (such as ppFEV1 or SCC values) between patients that received one or two treatments.

We quantified CFTR modulator responses in vitro by assessment of FIS of patient-derived rectal organoids that were previously cultured and stored in a biobank (Fig. 1a and 1b show an example, individual measurements for all patients are provided in supplemental Fig. 51). Organoid swelling was assessed after adding various concentrations of forskolin to facilitate optimal detection of drug response across the cohort for the various drugs. ${ }^{15}$ We used two outcome parameters to evaluate the in vivo clinical effect of a treatment: change in ppFEV1 and change in SCC. Pearson's correlations between organoid response and pulmonary response were analyzed in a subgroup of patients who had a ppFEV1 $\geq 40 \%$ and $\leq 90 \%$ before the start of treatment, to limit non-response of this endpoint (ceiling effects at $>90 \%$ or irreversible lung damage at $<40 \%$ ), as is usual in clinical trials. ${ }^{9,11,29-32}$ The organoid FIS positively correlated with both the pulmonary response (change in ppFEV1; $\mathrm{n}=21, \mathrm{r}=0.610, \mathrm{P}=0.003$, Fig. $1 \mathrm{c}$ ) and the change in $\mathrm{SCC}(\mathrm{n}=18, \mathrm{r}=-0.762, \mathrm{P}=<0.001$, Fig. $1 \mathrm{~d}$ ). As observed in other studies with CFTR modulators, the two in vivo endpoints appeared only weakly correlated, in a statistically non-significant manner (SCC vs ppFEV1, $n=18$, $r=-0.366$, $P=0.14$, Fig. 1e). We observed no big impact on the correlation of the repeated genistein plus curcumin and ivacaftor measurements; for ppFEV1: $n=21, r=0.624$, $\mathrm{P}=<0.001$ and for SCC: $\mathrm{n}=18, \mathrm{r}=-0.716, \mathrm{P}=<0.001$ (supplemental Fig. S2). In accordance with previous observations, all correlations were optimal when organoid responses at $0.128 \mu \mathrm{M}$ forskolin were used (supplemental Table S1). ${ }^{16}$ Patients with a ppFEV1 $>90 \%$ or ppFEV1 $<40 \%$ before the start of the treatment did not show a clear correlation between the organoid response and change in ppFEV1, despite an identical correlation between organoids and SCC (Fig. 1f,g)). The data of all patients combined showed correlations of organoids with both ppFEV1 ( $n=35, r=0.575, P=<0.001$, Fig. 1i) and SCC ( $n=33, r=-0.708$, $\mathrm{P}=<0.001$, Fig. 1j), but a statistically significant relation between ppFEV1 and SCC was not observed. (Fig. $1 \mathrm{~h}, \mathrm{k}$ ). People with rare mutations who were selected by organoids prior to treatment showed a median increase of $10 \%$ in ppFEV1 $(n=7, p=0.058)$ and a reduction of $39 \mathrm{mmol} / \mathrm{L}$ in SCC ( $\mathrm{n}=6, \mathrm{p}=0.028$ ). Collectively, these data demonstrates that in vitro CFTR modulator responses in organoids correlate with two important therapeutic endpoints. 


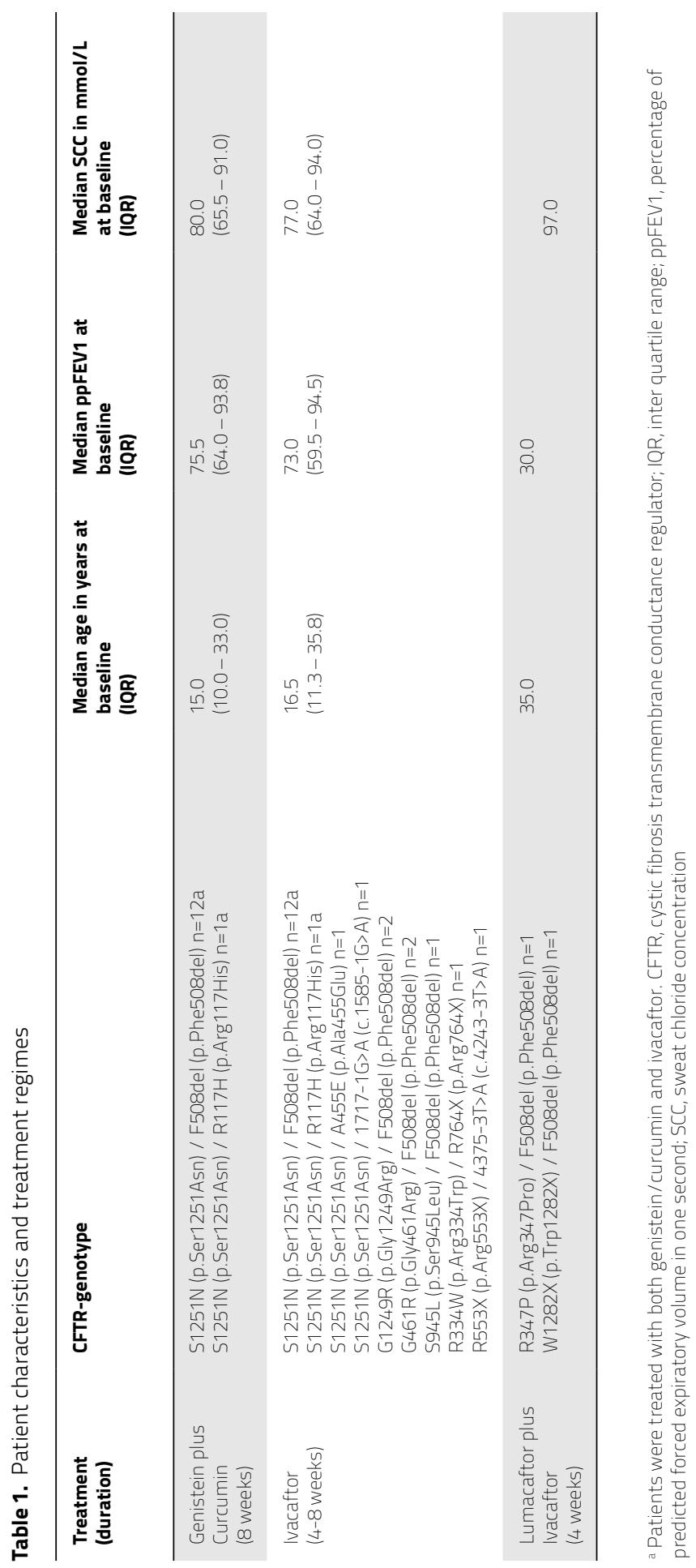




\section{Prediction of clinical responses using organoids}

Next, we generated ROC-curves to examine the predictive potential of different organoidbased thresholds for identifying clinical responders. We dichotomized both the ppFEV1 and SCC response into changes that are generally considered clinically significant and beyond the test variability (changes in ppFEV1 $>5 \%$, or SCC $>20 \mathrm{mM}$ or a combined change in ppFEV1>5\% and SSC $>20 \mathrm{mM}$ ) and changes that are not. ${ }^{33}$ The area under the ROCcurve provides a general measure for test accuracy and was 0.837 (95\% Cl $0.661-1.000)$ for predicting responders in ppFEV1 and increased towards 0.938 (95\% Cl $0.830-1.000$ ) for predicting responders in either SCC or SCC and ppFEV1 (Fig. 2a). When repeated measurements were taken into account, the area under the ROC-curve did not change. A Youden index was used to select an organoid cut-off point with the most optimal combination of sensitivity and specificity in an unbiased fashion. ${ }^{25}$ The selected cutoff value to identify responders in both SCC and ppFEV1 had a sensitivity of 0.80 and a specificity of 1.00 with a corresponding Youden index of 0.8 for identifying responders and non-responders in both ppFEV1 and SCC. The associated positive and negative predictive values were $100 \%$ and $80 \%$, respectively. Since data driven selection of the Youden index might cause over-estimation of both sensitivity and specificity, we performed a leaveone-out cross validation to further validate our findings. ${ }^{26}$ This additional analysis showed a sensitivity of 0.70 and specificity of 1.00 , with a corresponding Youden index of 0.70 .

For patients that started with a ppFEV1 $<40 \%$ or $>90 \%$ the ROC-curve had an area under the curve between 0.694 and 0.767 (Fig. 2b). For the total group of patients that was treated, the area under the ROC-curve varied between 0.783 and 0.869 (Fig. 2c). Because of the small sample size we did not calculate ROC-curves for the group of patients that had at least one rare CFTR mutation. In conclusion, the organoid-based test displayed excellent accuracy (AUC of ROC-curve > 0.9) for identifying clinical responses defined by changes in SCC and ppFEV1 or only SCC, while good accuracy (AUC of ROC-curve between 0.8 and 0.9 ) was observed for identifying clinical responses defined only by ppFEV1. ${ }^{34}$

\section{DISCUSSION}

This study aimed to provide evidence that FIS of rectal organoids can act as a prospective biomarker for in vivo CFTR modulator responses. We demonstrated here that individual in vitro CFTR modulator responses in these patient-derived stem cell cultures correlate with two independent indicators of therapeutic response in vivo. The moderate correlation between FIS and ppFEV1 and higher correlation between FIS and SCC (an in vivo biomarker of (FTR function) is in agreement with the higher impact of non-CFTR dependent factors on variation in pulmonary function as compared to SCC . 6,35 We did not find a statistically significant correlation between change in SCC and ppFEV1, probably because of a weaker 
Figure 1. Significant correlation between individual in vitro organoid response and in vivo change in ppFEV1 and SCC

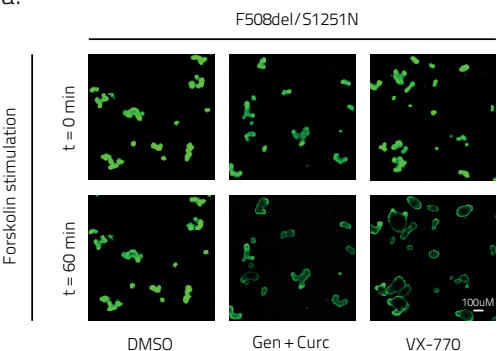

$\geq 40 \%$ and $\leq 90 \%$

c.

Organoids vs. FEV1 ( $\mathrm{n}=21$ )

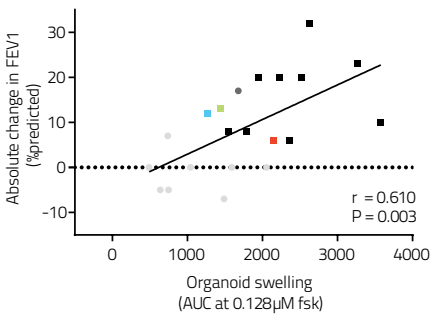

d

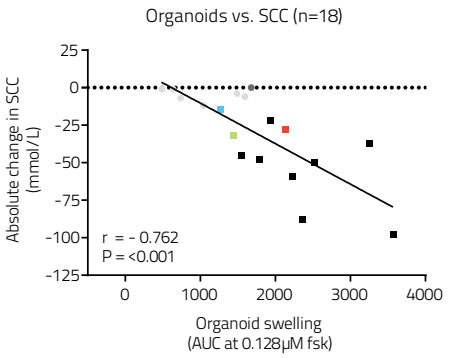

e.

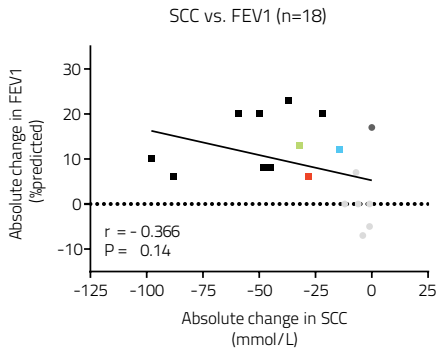

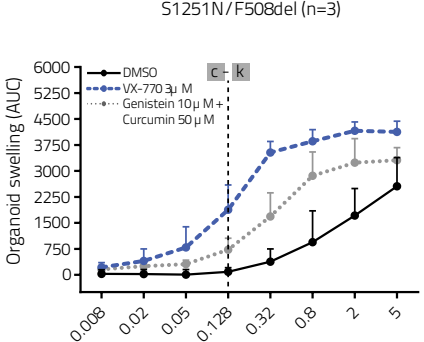

Forskolin $(\mu \mathrm{M})$

$$
<40 \% \text { or }>90 \%
$$

f.
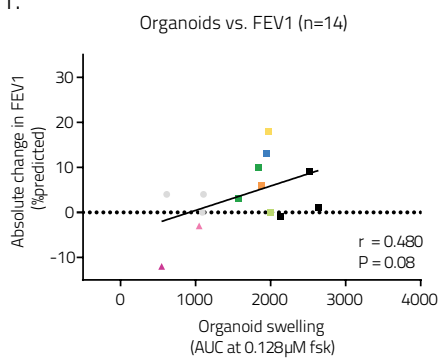

g.

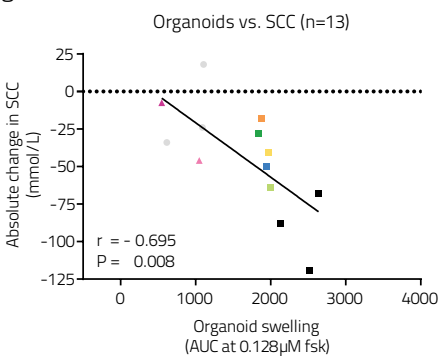

h.

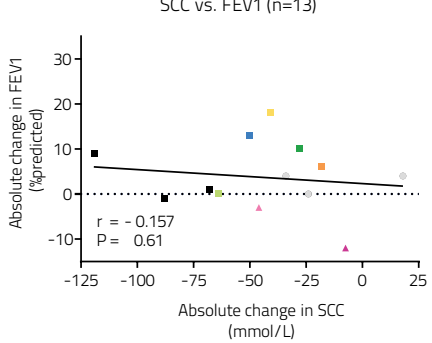


- Genistein + Curcumin in S1251N/F508de

- Genistein + Curcumin in S1251N/R117H

- $V X-770$ in $51251 \mathrm{~N} / \mathrm{F} 508 \mathrm{del}$

- $V X-770$ in $51251 \mathrm{~N} / \mathrm{R} 117 \mathrm{H}$

- VX-770 in S1251N/A455E

- VX-770 in $51251 \mathrm{~N} / 1717-1 \mathrm{G}>\mathrm{A}$

(- $v X-770$ in G1249R/F508del

- $V X-770$ in G461R/F508del

1. $V X-770$ in $R 553 X / 4375-3 T>A$

$V X-770$ in $5945 L / F 508 d$

- $V X-770$ in R334W/R764X

A. $V X-809+V X-770$ in $R 347 P / F 508 d e l$

A. $V X-809+V X-770$ in W1282X/F508de

\section{Total}

i.

Organoids vs. FEV1 $(n=35)$

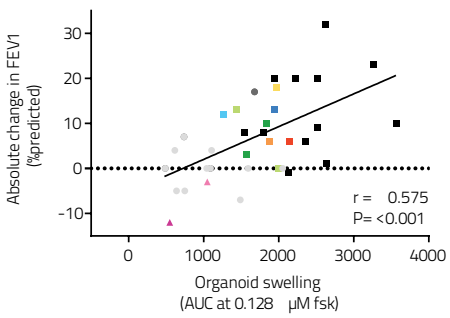

j.

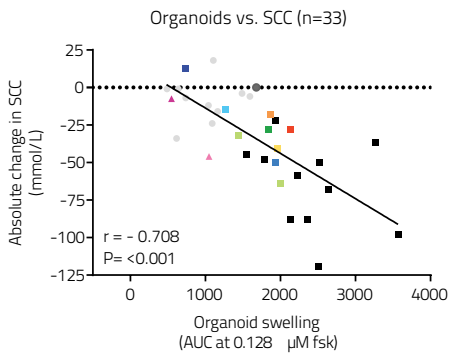

k.

SCC vs. FEV1 $(n=31)$

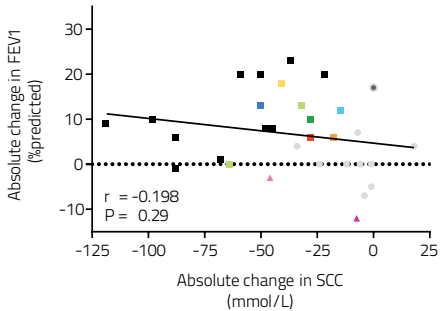

(a) Confocal images of the FIS of organoids with an F508del/S1251N mutation. Images are taken 0 and 60 minutes after adding DMSO, genistein plus curcumin and ivacaftor (VX-770) in combination with forskolin.

(b) AUC of theswelling of organoids after measuring for 60 minutes. The graph shows responses after adding eight different concentrations of forskolin in combination with either DMSO or a CFTR modulating treatment. Mean, \pm $5 \mathrm{D}$

$(\mathbf{c}, \mathbf{d})$ Pearson correlations between response of the organoids of an individual patient upon CFTR modulating treatment in combination with $0.128 \mu \mathrm{M}$ Forskolin and the in vivo response (change in ppFEV1 and change in SCC) of the same patient to the same treatment for patients who had a ppFEV $1 \geq 40 \%$ and $\leq 90 \%$ before the start of treatment.

(e) Pearson correlation between change in ppFEV1 and change in SCC of individual patients upon a CFTR modulating treatment for patients who had a ppFEV1 $\geq 40 \%$ and $\leq 90 \%$ before the start of treatment.

(f,g) Pearson correlations between response of the organoids of an individual patient upon CFTR modulating treatment in combination with $0.128 \mu \mathrm{M}$ Forskolin and the in vivo response (change in ppFEV 1 and change in SCC) of the same patient to the same treatment for patients who had a ppFEV $1<40 \%$ or $>90 \%$ before the start of treatment.

(h) Pearson correlation between change in ppFEV1 and change in SCC of individual patients upon a CFTR modulating treatment for patients who had a ppFEV1 $<40 \%$ or $>90 \%$ before the start of treatment.

(i,j) Pearson correlations between response of the organoids of an individual patient upon CFTR modulating treatment in combination with $0.128 \mu \mathrm{M}$ Forskolin and the in vivo response (change in ppFEV1 and change in SCC) of the same patient to the same treatment for all patients that received treatment.

(k) Pearson correlation between change in ppFEV1 and change in SCC of individual patients upon a CFTR modulating treatment for all patients that received treatment. 
Figure 2. Predicting individual clinical response by using rectal organoids of a patient

$$
\geq 40 \% \text { and } \leq 90 \%
$$

a.

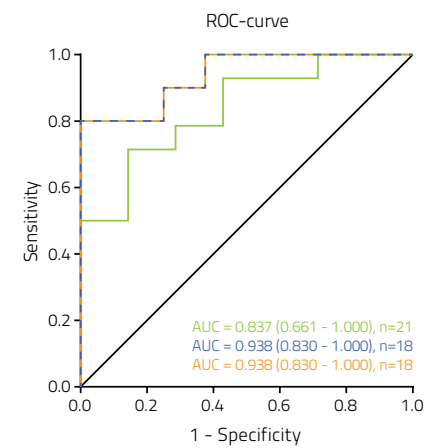

$<40 \%$ or $>90 \%$

b.

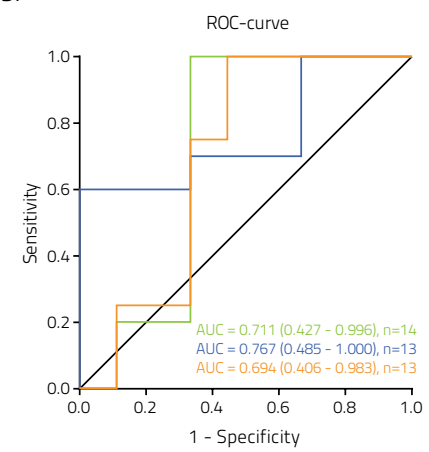

Total

C.

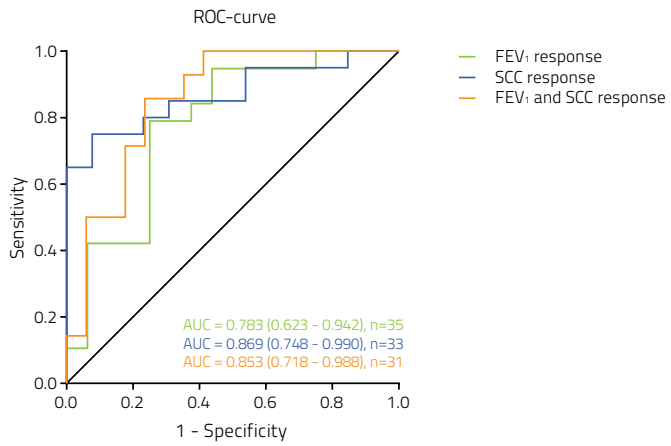

(a) ROC-curves of predicting which patient shows a response in ppFEV1, SCC and both ppFEV1 and SCC for patients who had a ppFEV1 $\geq 40 \%$ and $\leq 90 \%$ before the start of treatment.

(b) ROC-curves of predicting which patient shows a response in PpFEV1, SCC and both ppFEV1 and SCC for patients who had a ppFEV1 $<40 \%$ or $>90 \%$ before the start of treatment.

(c) ROC-curves of predicting which patient shows a response in PpFEV1, SCC and both ppFEV1 and SCC for all patients that received treatment.

correlation between these outcome measurements in combination with a small sample size, as was previously also observed in other studies with comparable sample sizes. ${ }^{36}$ These in vivo endpoints are suitable to indicate treatment effects at a group level, but non-CFTR dependent variation in PpFEV1 and SCC probably limits their precision and accuracy for informing on individual CFTR function modulation. ${ }^{37}$ In contrast, in vitro FIS is completely CFTR dependent, has sufficient sensitivity to quantitate CFTR modulator activity and the repeated measurements increase precision. These properties likely 
facilitate that FIS has sufficient accuracy to inform on both ppFEV1 and SCC (or their combination), suggesting that FIS is a potent biomarker to quantitate individual CFTR modulator responses.

Our dataset provides a first analysis of the predictive potential of the rectal organoids to identify clinical responders and non-responders to treatment. Our data support that FIS can be used to prospectively select responders and non-responders to CFTR modulator treatments but the cut-off value with the highest Youden index still needs to be interpreted carefully as well as the definition of clinical responders. The Youden index selects the most optimal ratio between sensitivity and specificity, but a different threshold with a higher negative predictive value may be preferential to limit the exclusion of treatment responders (e.g. an organoid threshold with a negative predictive value of $100 \%$ would have a positive predictive value of $77 \%$ ). Additionally, it remains unclear how short term treatment responses individually translate into long term clinical response. It could therefore be that the definitions for long term clinical responders are different, leading to other threshold values of predictive tests. We observed that the correlation of the organoid test with response in ppFEV1 was modified by baseline ppFEV1, despite similar correlation in SCC in both groups with differences in baseline ppFEV1. This supports that biomarkers of CFTR function such as organoid-based measurements have an important role for assessment of CFTR modulator responses in subjects where clinical domain indicators are unsuited to measure therapeutic response.

There are several limitations in this study. First, the open-label setting of treatments can induce bias in the acquisition of clinical data. Potentially ppFEV1 might have been influenced, but this is unlikely for SCC measurements. However, we do not expect that the open label setting has strongly affected the in vitro-in vivo correlation, since the clinical observers and patients were blinded for the in vitro drug responses and vice versa. Second, the study is biased for potentiator treatments. The area under the ROC-curves may be different when patients are stratified for different CFTR modulator treatments such as corrector/ potentiator combinations. Also the cut-off values of ppFEV1 and SCC that were used to define a clinical responder may not be fully accurate in identifying long-term clinical responders to treatment, and changing these cut-off values will lead to different ROC-curves. Third, patient subgroups with differences in organoid baseline CFTR functions may require different organoid test conditions (e.g. different forskolin conditions) for better predictive values. Fourth, it remains challenging to estimate adequate drug concentrations in the organoid tests as to optimally reflect the in vivo tissue concentration. For ivacaftor and lumacaftor we relied on average blood concentrations to determine the in vitro drug concentrations. ${ }^{38,39}$ For genistein and curcumin, lack of information on in vivo tissue concentrations may resulted in overdosing the in vitro situation, which can lead to overestimation of their potential in vivo effect. Most importantly, larger follow up studies remain needed to define more precisely how organoid-based measurements, and possibly other short term endpoints, can predict long term individual benefit to various CFTR modulator treatments. 
Apart from the performance of FIS as a biomarker of treatment response in this study, the rectal organoids provide additional benefits over other biomarkers of CFTR function. Rectal organoids are adult stem cell cultures that can be generated from a single rectal biopsy and cultured over 6 months while maintaining patient-specific CFTR modulator response. ${ }^{16,40}$ Rectal biopsies are accessible in most subjects independent of age and can be shipped to dedicated centers for organoid testing within weeks and stored in living biobanks which enables future drug testing. ${ }^{16}$ The FIS readout appears also not affected by CF disease phenotype (e.g. irreversible damage and inflammation in pulmonary markers). Currently, the immediate impact can be the selection of people for treatments independent of the CFTR genotype, both for CFTR modulators on the market and in development. For people having access to treatment, we may be able to further individually tailor treatments to maximize clinical benefits. ${ }^{41}$

\section{CONCLUSION}

In vitro drug efficacy measurements by FIS in rectal organoids of individuals with CF correlate with the most important in vivo response indicators of CFTR modulators (change in ppFEV1 and SCC). The data further suggest that thresholds can be established to prospectively identify clinical responders with acceptable positive and negative predictive values. Organoid testing can provide a patient-friendly and cost-effective approach to increase access to treatment for patients with CF, and optimize risk-benefit and cost-effectiveness of treatments. This study is a first example that in vitro tests using cultures of patient stem cells, stored in a living biobanks, can be used to predict individual treatment benefits.

\section{ACKNOWLEDGEMENTS}

We thank all patients who gave informed consent for generating and testing their individual organoids and the use of their data; all the members of the research teams that contributed to this work, especially E.M. Nieuwhof-Stoppelenburg, E.C. Kooij - van der Wiel and J.C. Escher (Department of Pediatric Pulmonology, Erasmus Medical Center/ Sophia Children's Hospital, Rotterdam, Netherlands), N. Adriaens and P.F.M. Mau Asam (Academic Medical Center, Amsterdam, Netherlands), M. Smink, I. Paalvast-Schouten and R.J.L. Stuyt (Haga Teaching Hospital, The Hague, Netherlands), S. Heida-Michel, M. Geerdink, I. Janse-Seip, H. van Panhuis, M.C.J. Olling-de Kok (Department of Pediatric Pulmonology, Wilhelmina Children's Hospital, University Medical Center Utrecht, Utrecht University, Utrecht, Netherlands), H. Oppelaar, M.C. Hagemeijer (Regenerative Medicine Center Utrecht, University Medical Center Utrecht, Utrecht University, Utrecht, 
Netherlands); and AOV for donating "AOV 811 curcuma longa" and "AOV 805 genistein" Funding: This work was supported by grants of the Dutch Cystic Fibrosis Foundation (NCFS) as part of the HIT-CF program and the Dutch Health Organization ZonMw, Netherlands.

\section{COMPETING INTERESTS}

J.F.D, H.C.C, J.M.B. and C.K.v.d.E are inventors on (a) patent(s) related to these findings. G.H.K, J.M.B. and C.K.v.d.E report receiving research grant(s) from Vertex Pharmaceuticals (money to institution), outside the submitted work. 


\section{REFERENCES}

1. Barker $\mathrm{N}$, van Es JH, Kuipers J, et al. Identification of stem cells in small intestine and colon by marker gene Lgr5. Nature. 2007;449(7165):1003-1007.

2. Sato T, Vries RG, Snippert HJ, et al. Single Lgr5 stem cells build crypt-villus structures in vitro without a mesenchymal niche. Nature. 2009;459(7244):262-265.

3. van de Wetering $M$, Francies HE, Francis JM, et al. Prospective derivation of a living organoid biobank of colorectal cancer patients. Cell. 2015;161(4):933-945.

4. Riordan JR, Rommens JM, Kerem B, et al. Identification of the cystic fibrosis gene: cloning and characterization of complementary DNA. Science. 1989;245(4922):1066-1073.

5. Sosnay PR, Siklosi KR, Van Goor F, et al. Defining the disease liability of variants in the cystic fibrosis transmembrane conductance regulator gene. Nat Genet. 2013;45(10):11601167.

6. Cutting GR. Cystic fibrosis genetics: from molecular understanding to clinical application. Nat Rev Genet. 2014;(November).

7. Dekkers JF, Van Mourik P, Vonk AM, et al. Potentiator synergy in rectal organoids carrying S1251N, G551D, or F508del CFTR mutations. J Cyst Fibros. 2016;15(5).

8. Ramsey BW, Davies J, McElvaney NG, et al. A CFTR potentiator in patients with cystic fibrosis and the G551D mutation. N Engl J Med. 2011;365(18):1663-1672.

9. De Boeck K, Munck A, Walker S, et al. Efficacy and safety of ivacaftor in patients with cystic fibrosis and a non-G551D gating mutation. J Cyst Fibros. 2014;13(6):674-680.

10. Wainwright CE, Elborn JS, Ramsey BW, et al. Lumacaftor-Ivacaftor in Patients with Cystic Fibrosis Homozygous for Phe508del CFTR. N Engl J Med. May 2015.

11. Moss RB, Flume PA, Elborn JS, et al. Efficacy and safety of ivacaftor in patients with cystic fibrosis who have an Arg117His-CFTR mutation: a double-blind, randomised controlled trial. Lancet Respir Med. 2015;3(7):524-533.

12. Rowe SM, Daines C, Ringshausen FC, et al. Tezacaftor-Ivacaftor in Residual-Function Heterozygotes with Cystic Fibrosis. N Engl J Med. November 2017:NEJMoa1709847.

13. Taylor-Cousar JL, Munck A, McKone EF, et al. Tezacaftor-Ivacaftor in Patients with Cystic Fibrosis Homozygous for Phe508del. N Engl J Med. November 2017:NEJMoa1709846.

14. Ratner M. FDA deems in vitro data on mutations sufficient to expand cystic fibrosis drug label. Nat Biotechnol. 2017;35(7):606.

15. Dekkers JF, Wiegerinck CL, de Jonge HR, et al. A functional CFTR assay using primary cystic fibrosis intestinal organoids. Nat Med. 2013;19(7):939-945.

16. Dekkers JF, Berkers G, Kruisselbrink E, et al. Characterizing responses to CFTR-modulating drugs using rectal organoids derived from subjects with cystic fibrosis. Sci Transl Med. 2016;8(344).

17. Zomer-van Ommen DD, Vijftigschild LAW, Kruisselbrink E, et al. Limited premature termination codon suppression by read-through agents in cystic fibrosis intestinal organoids. J Cyst Fibros. 2016;15(2):158-162.

18. Zainal Abidin N, Haq IJ, Gardner Al, Brodlie M. Ataluren in cystic fibrosis: development, clinical studies and where are we now? Expert Opin Pharmacother. August 2017:1-9.

19. de Winter-de Groot KM, Janssens HM, van Uum RT, et al. Stratifying infants with cystic fibrosis for disease severity using intestinal organoid swelling as a biomarker of CFTR function. Eur Respir J. 2018;52(3):1702529.

20. Sato $T$, Stange DE, Ferrante M, et al. Long-term Expansion of Epithelial Organoids From Human Colon, Adenoma, Adenocarcinoma, and Barrett's Epithelium. Gastroenterology. 2011;141(5):1762-1772.

21. Dutch Cystic Fibrosis Foundation. Nederlandse Cystic Fibrosis Registratie 2015. Ned Cyst Fibros Regist. 2016. https://www.ncfs.nl/bestanden/cf-registratie/rapportage2015.pdf. 
22. Boj SF, Vonk AM, Statia M, et al. Forskolin-induced Swelling in Intestinal Organoids: An \&lt;em\&gt;In Vitro\&lt;/em\&gt; Assay for Assessing Drug Response in Cystic Fibrosis Patients. J Vis Exp. 2017;(120).

23. Standardization of Spirometry, 1994 Update. American Thoracic Society. Am J Respir Crit Care Med. 1995;152(3):1107-1136.

24. Beydon N, Davis SD, Lombardi E, et al. An official American Thoracic Society/European Respiratory Society statement: pulmonary function testing in preschool children. Am J Respir Crit Care Med. 2007;175(12):1304-1345.

25. YOUDEN WJ. Index for rating diagnostic tests. Cancer. 1950;3(1):32-35.

26. Leeflang MMG, Moons KGM, Reitsma JB, Zwinderman AH. Bias in sensitivity and specificity caused by data-driven selection of optimal cutoff values: mechanisms, magnitude, and solutions. Clin Chem. 2008;54(4):729-737.

27. Lorenz DJ, Datta S, Harkema SJ. Marginal association measures for clustered data. Stat Med. 2011;30(27):3181-3191.

28. Obuchowski NA. Nonparametric analysis of clustered ROC curve data. Biometrics. 1997;53(2):567-578.

29. Ramsey BW, Davies J, McElvaney NG, et al. A CFTR Potentiator in Patients with Cystic Fibrosis and the G551D Mutation. N Engl J Med. 2011;365(18):1663-1672.

30. Wainwright CE, Elborn JS, Ramsey BW, et al. Lumacaftor-Ivacaftor in Patients with Cystic Fibrosis Homozygous for Phe508del CFTR. N Engl J Med. 2015:150517100015004.

31. Wood ME, Smith DJ, Reid DW, Masel PJ, France MW, Bell SC. Ivacaftor in severe cystic fibrosis lung disease and a G551D mutation. Respirol case reports. 2013;1(2):52-54.

32. Taylor-Cousar JL, Jain M, Barto $\mathrm{TL}$, et al. Lumacaftor/ivacaftor in patients with cystic fibrosis and advanced lung disease homozygous for F508del-CFTR. J Cyst Fibros. November 2017.

33. Seliger VI, Rodman D, Van Goor F, Schmelz A, Mueller P. The predictive potential of the sweat chloride test in cystic fibrosis patients with the G551D mutation. J Cyst Fibros. 2013;12(6):706-713.

34. Metz CE. Basic principles of ROC analysis. Semin Nucl Med. 1978;8(4):283-298.

35. Collaco JM, Blackman SM, Raraigh KS, et al. Sources of Variation in Sweat Chloride Measurements in Cystic Fibrosis. Am J Respir Crit Care Med. June 2016.

36. Accurso FJ, Rowe SM, Clancy JP, et al. Effect of VX-770 in persons with cystic fibrosis and the G551D-CFTR mutation. N Engl J Med. 2010;363(21):1991-2003.

37. Fidler MC, Beusmans J, Panorchan P, Van Goor F. Correlation of sweat chloride and percent predicted FEV1 in cystic fibrosis patients treated with ivacaftor. J Cyst Fibros. October 2016.

38. Summary of Product Characteristics - Kalydeco. 2018:1-76. https://www.ema.europa.eu/ documents/product-information/kalydeco-epar-product-information_en.pdf.

39. Summary of Product Characteristics - Orkambi.; 2018. https://www.ema.europa.eu/ documents/product-information/orkambi-epar-product-information_en.pdf.

40. Clevers H. Modeling Development and Disease with Organoids. Cell. 2016;165(7):15861597.

41. Beekman JM. Individualized medicine using intestinal responses to CFTR potentiators and correctors. Pediatr Pulmonol. 2016;51(S44):S23-S34. 


\section{SUPPLEMENTALS}

Supplemental Figure S1. Individual organoid measurements of all patients that received treatment
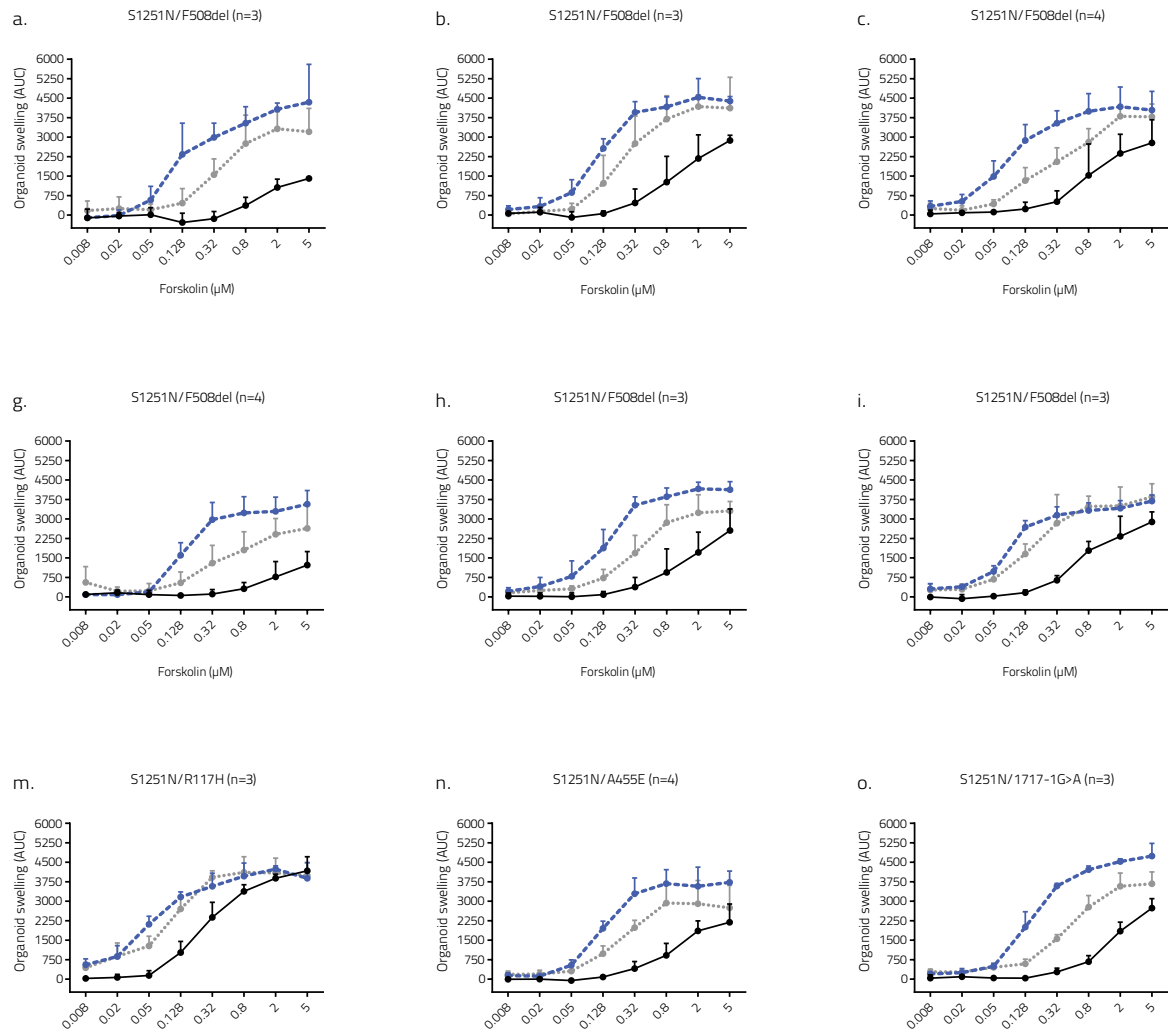

Forskolin $(\mu \mathrm{M})$

Forskolin ( $\mu \mathrm{M})$

Forskolin (HM)
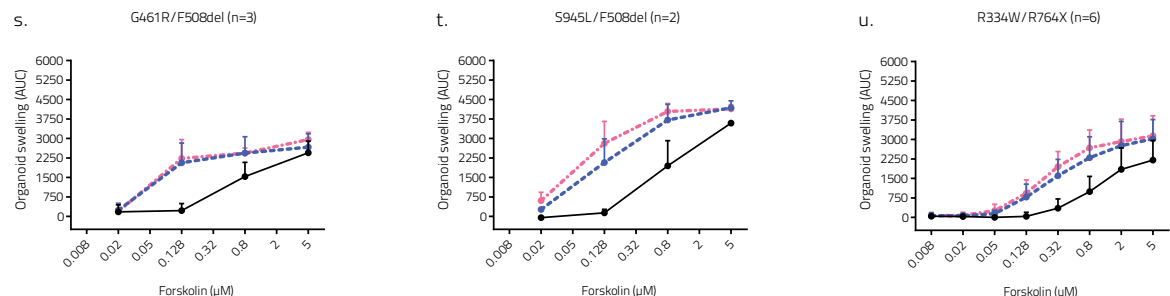

(a-x) Area Under the Curve (AUC) of the swelling of organoids of all individual patients after measuring for 60 minutes. The graphs show responses after adding four to eight different concentrations of forskolin in combination with either DMSO or a CFTR modulating treatment. Mean, \pm SD. (h) Was also used as an example in Fig $1 \mathrm{~b}$. 

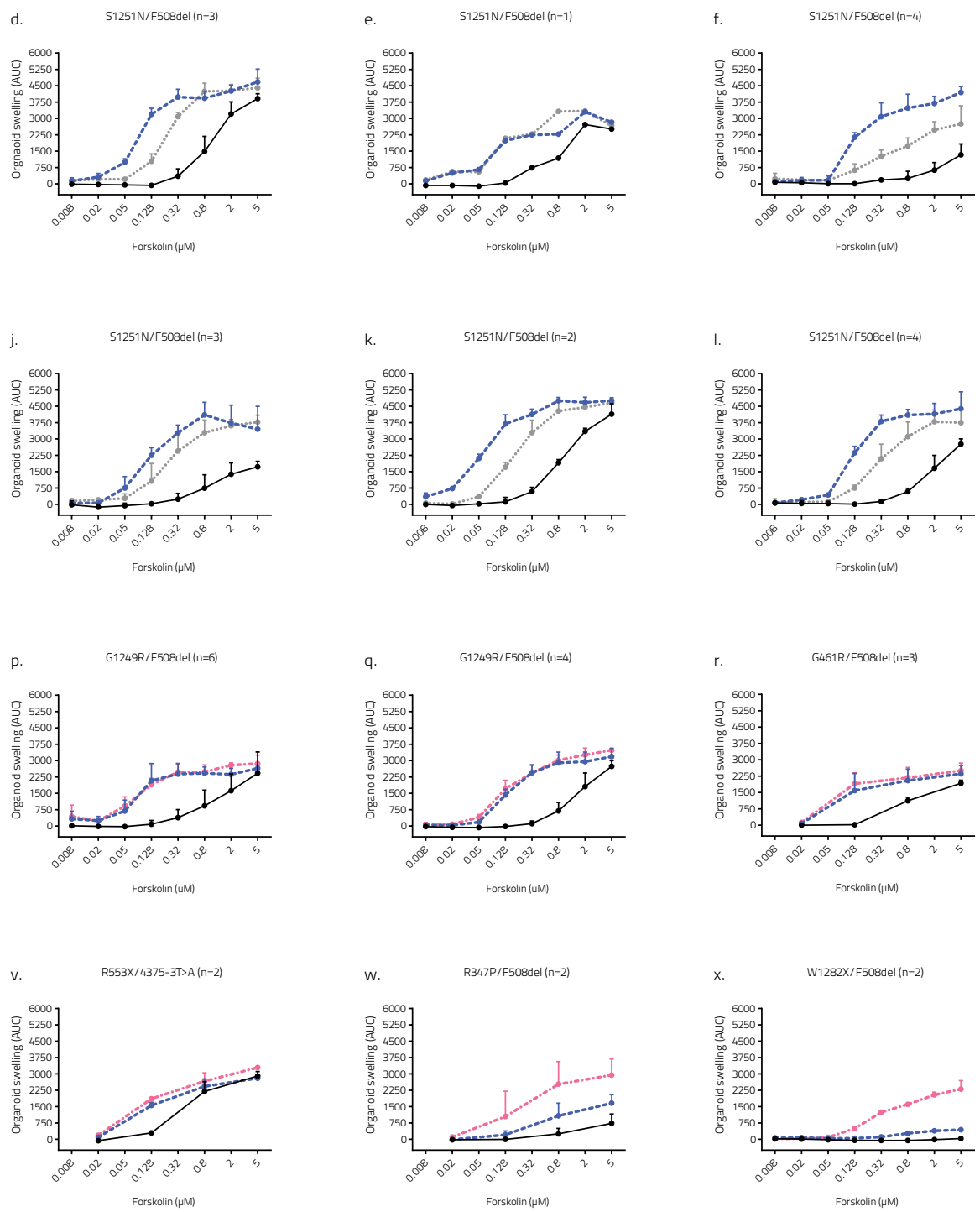

$\rightarrow$ DMSO

-..-. $v X-7703 \mu \mathrm{M}$

........ Genistein $10 \mu \mathrm{M}+$ Curcumin $50 \mu \mathrm{M}$

$\ldots . . . \quad V X-8093 \mu M+V X-7703 \mu M$ 
Supplemental Figure S2. Correlation between individual in vitro organoid response and in vivo change in PpFEV1 and SCC, controlled for repeated measurements

a.

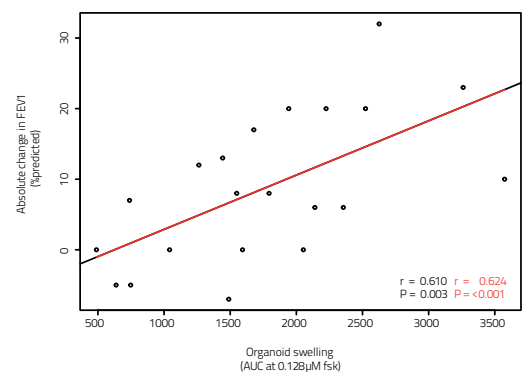

b.

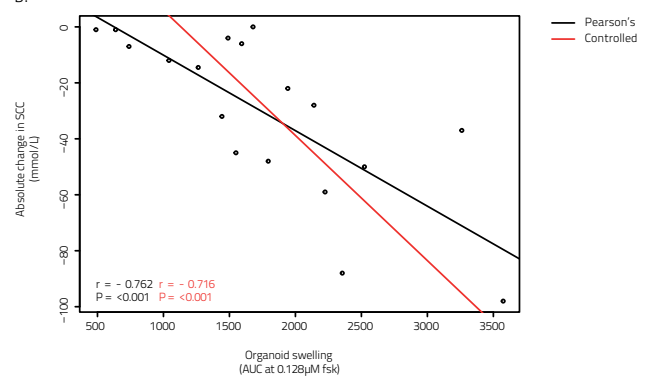

$(\mathbf{a}, \mathbf{b})$ Correlations between response of the organoids of an individual patient upon CFTR modulating treatment in combination with $0.128 \mu \mathrm{M}$ Forskolin and the in vivo response (change in ppFEV1 and change in SCC) of the same patient to the same treatment for patients who had a ppFEV1 $\geq 40 \%$ and $\leq 90 \%$ before the start of treatment, controlled for repeated measurements. 


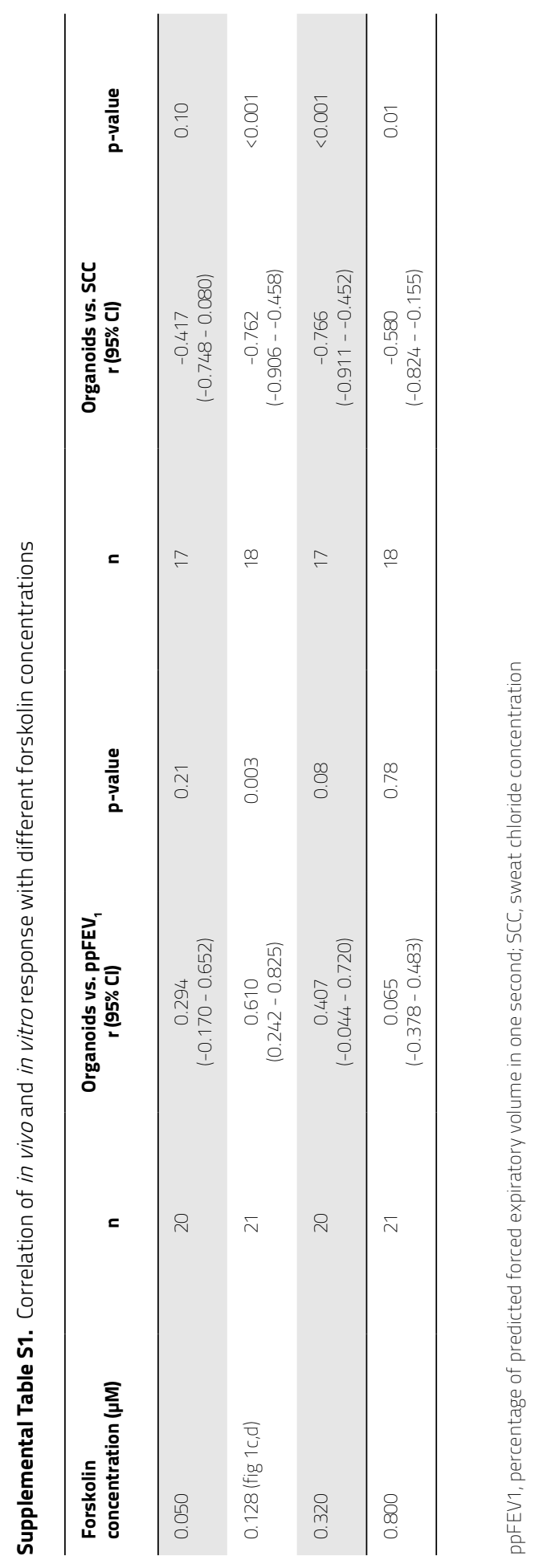


Supplemental Table S2. Key resources table

\section{REAGENT or RESOURCE}

SOURCE

IDENTIFIER

\section{Biological Samples}

Human rectal tissue

This paper

http://hub4organoids.eu/

\section{Chemicals, Peptides, and Recombinant Proteins}

B27 supplement with Vitamin A

Thermo Fisher Scientific: Cat\# 17504-044 Invitrogen

\begin{tabular}{lll}
\hline N-Acetylcysteine & Sigma Aldrich & Cat\# A9165-25g \\
\hline Nicotinamide & Sigma Aldrich & Cat\# N0636 \\
\hline Mouse Epithelial Growth Factor & Invitrogen & Cat\# PMG8043-1mg \\
\hline TGFb type I Receptor inhibitor (A83-01) & Tocris & Cat\# 2939 \\
\hline p38 MAPK inhibitor (SB202190) & Sigma Aldrich & Cat\# S7067-25mg \\
\hline Calcein, AM & Life Technologies: Gibco & Cat\# C3100MP \\
\hline Forskolin & Sigma & Cat\# F3919-10mg \\
\hline Lumacaftor (VX-809) & Selleckchem & Cat\# 51565 \\
\hline Ivacaftor (VX-770) & Selleckchem & Cat\# 51144 \\
\hline Genistein & Sigma & Cat\# 92136-10mg \\
Curcumin & Sigma & Cat\# C7727-500mg
\end{tabular}

\section{Deposited Data}

CFTR2 database

Johns Hopkins University /

https://www.cftr2.org/

Hospital for Sick Children /

CF Foundation

\section{Experimental Models: Cell Lines}

\section{Human rectal organoid lines}

$\mathrm{L}-$ Wnt $3 \mathrm{~A}$ producing cell line

Hek293T - Noggin hFc cell line

Hek293T - R-spondin-1 mFc cell line
This paper

http://hub4organoids.eu/

http://hub4organoids.eu/

http://hub4organoids.eu/

Trevigen Cat\# 3710-001-K


REAGENT or RESOURCE

\section{Software and Algorithms}

Zen Image analysis software module

SPSS

IBM

R-studio

Graphpad prism
SOURCE

Zeiss

https://www.rstudio.com/

Graphpad
IDENTIFIER

https://www.zeiss.com/ microscopy/int/products/ microscope-software/zen/ image-analysis.html

https://www.ibm com/analytics/nl/nl/ technology/spss/

https://www.rstudio.com/

https://www.graphpad. com/scientific-software/ prism/

\section{Other}

Matrigel $\circledast$ (protein concentration between 9.8$10.2 \mathrm{mg} / \mathrm{ml}$ ) 


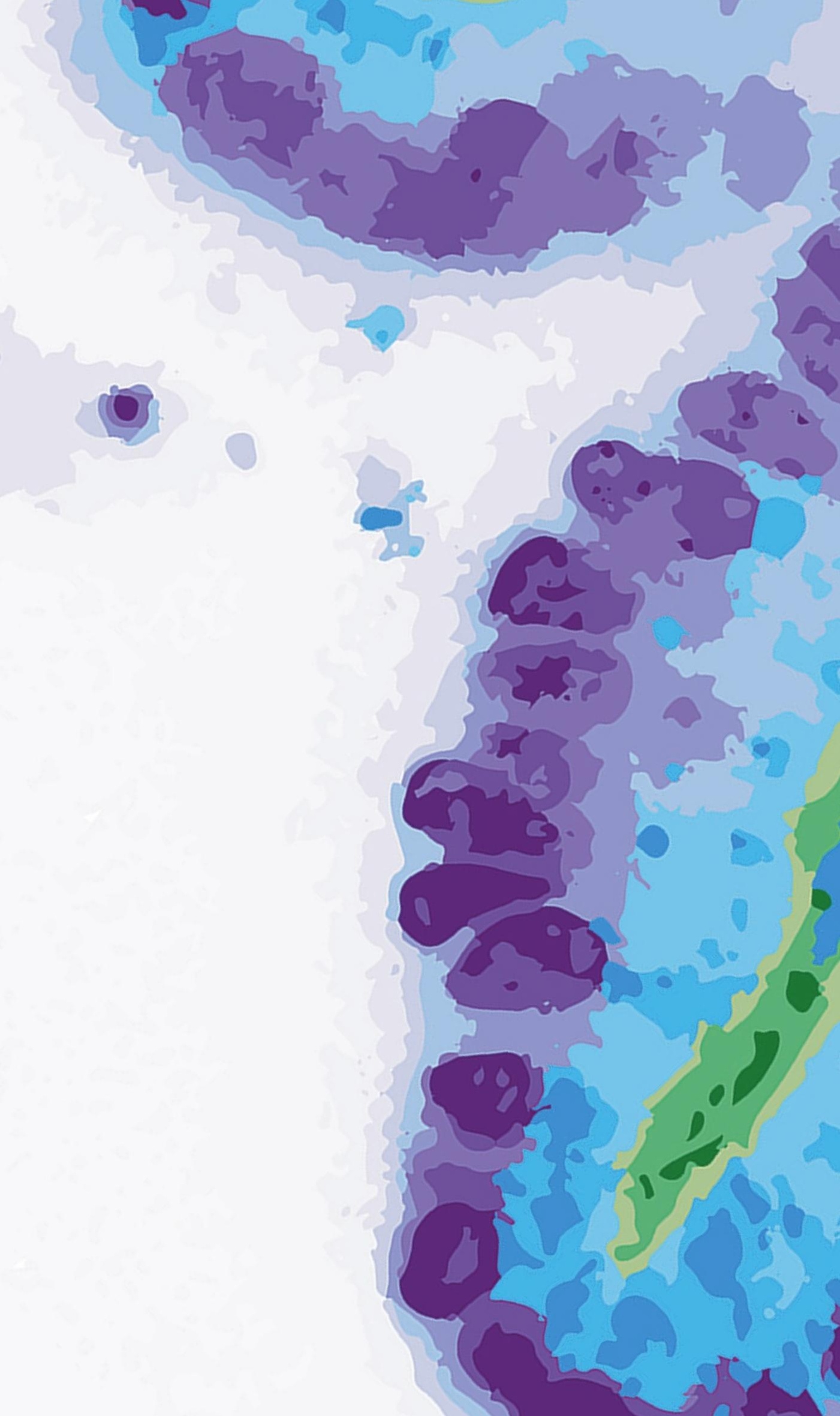




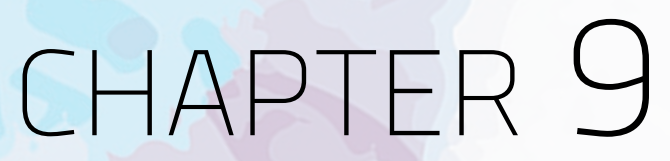

GENERAL DISCUSSION 
In this thesis we show that the intestinal organoid model enabled us to successfully select individual patients with very rare mutations for a clinically effective treatment with a cystic fibrosis transmembrane conductance regulator (CFTR) modulator. Furthermore we show successful examples but also challenges that associate with translating preclinical organoid responses to a clinical setting. In this chapter we discuss how we can use the model:

I. to screen for new and effective CFTR modulating drugs for patients with different CFTR mutations

II. as a tool for personalized treatment with CFTR modulators, especially in patients with a rare CFTR mutation. 


\section{ORGANOIDS AS A DRUG SCREENING TOOL}

\section{Organoids as a tool to predict clinical effect of CFTR modulators in groups of patients}

It is important to know whether measurements in the intestinal organoids reflect the in vivo characteristics of the population that the organoids represent, to be able to determine the utility of the organoid model. This clinical validation has to be performed for both clinical disease severity and drug response.

A first study on the relation between residual in vitro CFTR function and a patients' phenotype showed successful identification of subgroups that were different in both pulmonary and gastrointestinal outcome parameters.1 In this thesis (chapter 7) we confirm that the forskolin-induced swelling (FIS) of intestinal organoids can be used to evaluate residual CFTR function and identify subgroups that are known to be different (based on literature) with regards to their residual CFTR function. The correlation between in vitro CFTR improvement in response to a drug and the in vivo treatment effect on

Figure 1. Correlation between average drug response in organoids and clinical response of patients with the same mutation.

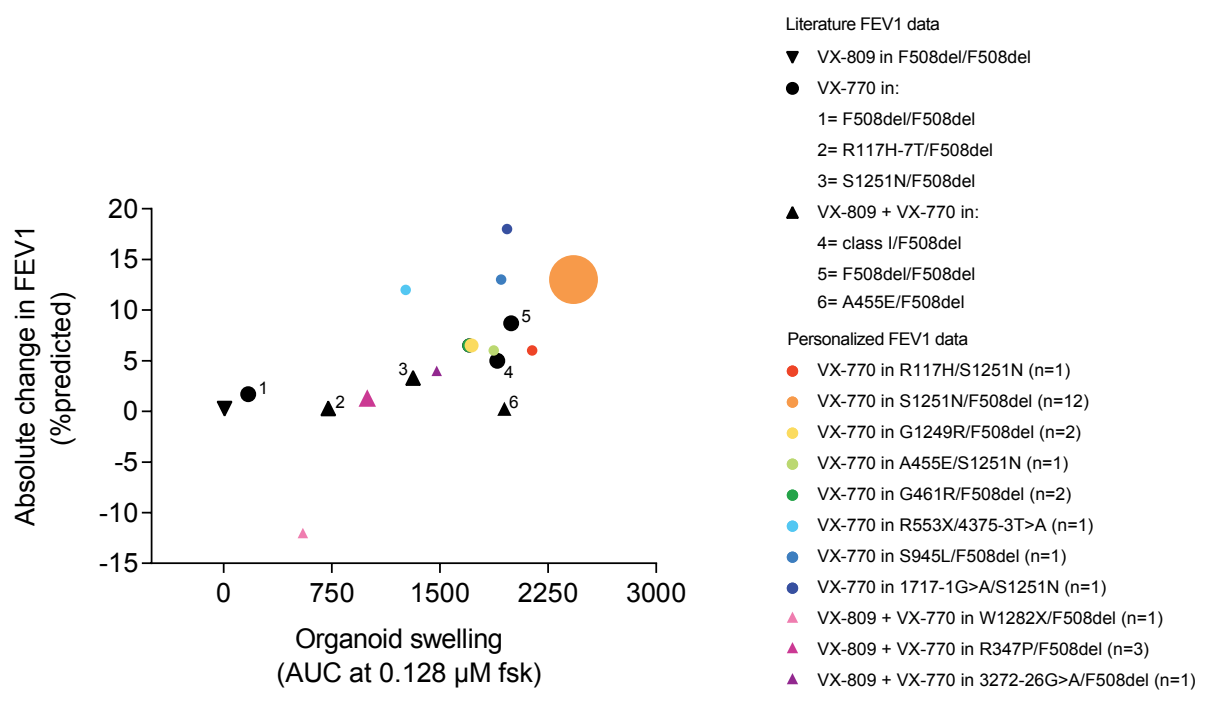

Organoid responses to a drug are measured in combination with $0.128 \mu \mathrm{M}$ Forskolin corrected for the dimethylsulphoxide (DMSO) response. Mean response of organoid from 3-8 patients for literature data and 1-12 patients for personalized data. Clinical response evaluated after 4-24 weeks of treatment with a drug. ${ }^{2-7}$ In personalized data larger dots represent clinical response of more patients. 
group level is shown in Figure 1 and includes all data that is currently available at our site. This relation is important to determine whether if we can use the organoid model for drug screening.

In this thesis we created several datasets, presented in Figure 1, to determine the correlation between in vitro organoid responses and clinical outcome in several groups of patients. Chapter 7 shows that the response of intestinal organoids to the CFTR modulators ivacaftor and ivacaftor+lumacaftor correlates with the results of the clinical trials in which patients with the same mutation were treated with these compounds. Despite the fact that FIS and clinical response were not evaluated in the same patient, these results suggest that there is a clear relation between the response of intestinal organoids to CFTR modulators and the clinical response to these drugs, when these responses are matched on CFTR genotype. In chapter 3 we evaluated the FIS of intestinal organoids of patients with a $51251 \mathrm{~N}$ gating mutation upon three different potentiator treatments (curcumin, genistein and ivacaftor) and the relation with the clinical response of the patient to these treatments, evaluated in three clinical trials. In organoids we found an increasing potentiator effect of a treatment with curcumin + genistein, ivacaftor and ivacaftor + genistein respectively but the in vivo results only showed a clear clinical effect of ivacaftor therapy with a median improvement in percent predicted forced expiratory volume in one second $\left(\mathrm{ppFEV}_{1}\right.$ ) of $9.5 \%$. For ivacaftor therapy the plasma concentration of the compounds that was reached during the treatments was comparable with the concentration that was used in the in vitro setting, but for curcumin and genistein the reached concentrations during treatment were not even near the concentration that was used in vitro. This might explain the lack of in vivo efficacy of treatment with these food supplements and also shows the importance of implementing pharmacokinetic properties of a drug in the in vitro setting. In chapter 5 we repurposed the ivacaftor+lumacaftor therapy for a group of patients with the more rare A455E CFTR mutation. Chapter 5 showed that in patients with A455E mutations the association between in vitro and in vivo outcomes was somewhat different compared to the data found in earlier studies with patients who had for example an S1251N or S1249N mutation and were treated with ivacaftor (Fig.1). This raises the issue that we should be cautious with extrapolating the clinical outcomes with a certain study drug in a certain population to populations with other genotypes, or to patients who are treated with other drugs. Differences in dose-response relationships and differences in pharmacokinetics both deserve further studies.

Also differences in clinical study design might be responsible for differences found between various in vitro - in vivo relationships described in literature. The first clinical trials on the effect of potent CFTR modulators usually evaluate the clinical effect of a treatment after a couple of weeks. ${ }^{5,6,8-10}$ The importance of collecting data on the long term effects of these treatments is highlighted by the results of two years of treatment with ivacaftor in G551D patients. These data show that patients without short-term benefit on 
ppFEV ${ }_{1}$ may still have a long term efficacy with regards to exacerbation rate and ppFEV decline. ${ }^{11}$ Moreover, long term data on the use of ivacaftor and ivacaftor+lumacaftor in patients with other CFTR mutations show a reduced decline of ppFEV 1 versus matched controls. ${ }^{12,13}$ However, patients still show a decline in $\mathrm{ppFEV}$, which suggests that CFTRfunction is not on the level of a healthy control, despite the clear improvement in PpFEV, after the first weeks of treatment. $3,5,7,14$

The finding of clear in vitro - in vivo correlations can be very helpful to support evidence for efficacy in small patient populations, which is often the case in rare diseases like cystic fibrosis (CF). The results of chapter 5 illustrate the difficulty of performing clinical trials in small groups of patients, since one or two outliers or drop-outs can have a big impact on the results of the trial. Introducing a more flexible wash-out period (with a minimum and maximum duration) might prevent drop-outs due to an exacerbation in the wash-out period, since patients have the time to recover from their exacerbation and are still eligible to be treated during the second treatment period. Moreover, in a small group of patients a study design with four instead of two treatment periods could minimize the effect of outliers, since both treatment and placebo effect are evaluated twice in each patient.

In conclusion, the results on the relation between intestinal organoid measurements and clinical parameters on group level show that there is a clear relation between CFTR function measured in organoids and both clinical disease severity and drug response.

\section{The use of organoids for drug screening}

Many therapeutics that have promising results in vitro fail in the clinical phase, which is called 'the valley of death'. ${ }^{15}$ Clinically validated in vitro models like patient derived intestinal organoids might be valuable tools for drug screening of currently available drugs and also for future drug testing. The human and personalized origin of this model as well as the ability to generate organoids from a single rectal biopsy, culture them indefinitely and store them in a living biobank, makes this model a promising tool for high throughput drug screening. The current "gold standard" for preclinical drug testing are cultures from human bronchial epithelial (HBE) cells of which drug responses to modulators correlate well with the mean improvement of a patient group in vivo.16 The unlimited culture and store options of intestinal organoids are a major advantage compared to the HBE cells that do not have these properties. Besides that, intestinal tissue is more easy to collect from a patient than HBE cells, which are cultures from explanted HBE from patients who undergo lung transplantation.

One of the first indications that organoids can be used to test efficacy of upcoming therapies was shown with PTC124 (ataluren). Based on in vitro results, this drug was proposed to be effective as read-through agent in nonsense mutations in the CFTR 
gene, but in vitro finding in organoids did not show any effect on CFTR function in the organoids of patients with a nonsense mutation. ${ }^{17} \mathrm{~A}$ first clinical Phase III study with this compound failed to show efficacy, especially in patients who were on co-medication with tobramycin. ${ }^{18}$ Subsequently, also a second Phase III study in tobramycin-negative subjects failed to show any efficacy. ${ }^{19}$

In this thesis (chapter 2, 3 and 5) we show the results of multiple drug screens in the intestinal organoid assay, as well as the evaluation of the clinical effect of the selected drugs. This demonstrates the power of the model as a tool for screening new drugs to modify CFTR function.

Screening the effect on CFTR function of large numbers of drugs in organoids of large number of patients with different mutations introduces a need for a high throughput screen (HTS). An automatized HTS setting in 384-well plates is currently being performed in a screen in which the CFTR modulating effect of 1400 FDA approved drugs is evaluated in organoids from more than 100 patients with rare CFTR mutations. When reliable CFTR function measurements can be performed by using the HTS, this will be a more efficient assay to screen for effective CFTR modulating drugs than the manual procedures that are being performed in a 96-well setting. The HTS may then not only be used to identify new CFTR modulator treatments but also to test combinations of available CFTR modulators from different pharmaceutical companies.

A drawback of the model is the lack of measuring pharmacokinetics in the model, because the concentrations used in vitro cannot always be reached in the in vivo clinical setting, as shown in chapter 3 of this thesis. If no pharmacokinetic data is available, a pilot treatment of a patient with a selected drug can be performed to determine for example plasma concentration that is reached during treatment. In chapter 3 we also show that the plasma of a patient that is collected during treatment can be used to evaluate the effect of the reached plasma concentration in the intestinal organoid model. It could be that the optimal assay conditions to predict clinical efficacy differ not only between different drugs but also between groups of patients with different residual CFTR function. When more data is available, additional analysis on the correlation between in vitro and in vivo response of patients to different drugs should be performed. For example, separate correlation plots should not only be generated for patient groups with differences in their residual CFTR function but potentially also for different (classes of) drugs. 


\section{ORGANOIDS AS A TOOL FOR PERSONALIZED MEDICINE}

\section{Organoids as a tool to predict clinical effect of CFTR modulators in individual patients}

Collecting data on the correlation between organoid response and clinical response in groups of patients with the same mutation is a first step in the clinical validation process of the intestinal organoid model. However, to know if organoids can be used to facilitate personalized medicine, more information on the relation between in vitro and in vivo response in individual patients is needed.

Previous publications on the correlation between a patients' residual CFTR function evaluated with FIS and the intestinal current measurement (ICM) and sweat chloride concentration (SCC) values of a patient show significant correlations between FIS and both outcome parameters, in patients with different CFTR mutations. ${ }^{1,20}$ In chapter 6 we show that also for patients with the same CFTR mutation, there is a significant correlation between the FIS of a patients' organoid and important pulmonary and nutritional parameters. In chapter 7 we show the first case of an organoid based selection of an individual patient with a rare CFTR mutation, for treatment with a CFTR modulator. The correlation between the response of a patients' organoid to CFTR modulating treatment and the clinical response of the same patient to the same drug was further evaluated in chapter 8. In this chapter we show that there is a significant correlation between FIS response to a CFTR modulator and change in both $\mathrm{pPFEV}_{1}$ and SCC for an individual patient.

The correlations that we found in chapter 8 are the basis for using the organoid model for personalized medicine. These correlations were used to generate receiver operating characteristic (ROC) curves and evaluate the predictive characteristics of the organoid model, when it is used to select a responder to a CFTR modulator.

To be able to generate an ROC-curve, we had to define which patient was "a clinical responder" based on change in $\mathrm{ppFEV}_{1}$ and/or SCC. A definition that is subject to discussion for both parameters. Change in $\mathrm{ppFEV}_{1}$ is often the main outcome parameter in clinical studies with CFTR modulators, and also the main outcome parameter upon which regulatory authorities such as the American food and drug administration (FDA) and the European medicines agency (EMA) base their decision whether or not a drug is effective. Besides the previously discussed point that short term change in ppFEV, does not always predict long term treatment effect, determining the response to a CFTR modulator of an individual patient also introduces other challenges. ${ }^{11}$ For example, not only the function of the CFTR protein determines pulmonary disease and thus response to a modulator, but also other factors such as environmental factors or damage of the lung tissue at baseline have an effect on a patients' pulmonary response to a modulator. ${ }^{21}$ Also for the sweat test it is known that not only CFTR function has an effect 
on the chloride concentration, but that other factors such as test variability and biological variability over time also cause variability in the sweat chloride concentration. ${ }^{22,23}$ The factors that influence the result of a measurement other than CFTR function, could have a big impact on the conclusion on drug efficacy, especially in an individual patient.

The challenges in determining a clinical response for an individual patient are also illustrated by the finding that there is no significant correlation between change in ppFEV, and SCC, nasal potential difference (NPD) and ICM response in patients homozygous for the F508del mutation that were treated with ivacaftor+lumacaftor. ${ }^{24}$ Moreover, when the correlation between change in PpFEV $_{1}$ and SCC upon CFTR modulator treatment is calculated in patients with different mutations and treatments, it is found that the response of SCC to a drug is predictive for the change in $\mathrm{ppFEV}_{1}$ on a population basis but not for individual patients. ${ }^{25}$ These findings are supported by our results in chapter 8 that show a moderate correlation between change in ppFEV ${ }_{1}$ and SCC. In chapter 4 we evaluated the changes of a potential clinical marker for gastrointestinal problems upon treatment with a CFTR modulator. We found that these two biomarkers (fibroblast growth factor 19 and -hydroxy-4-cholesten-3-one (FGF19 and (4)) improved significantly after treatment with a CFTR modulator on a group level, but did not show a clear correlation with change in SCC and ppFEV for the individual patient. Not only the lack of correlation between change in these parameters of gastrointestinal function and SCC and PpFEV ${ }_{1}$ but also the moderate correlation between change in SCC and ppFEV1 found in chapter 8 illustrate the difficulties in determining the clinical response of an individual patient. Despite the limited precision and accuracy of SCC and ppFEV 1 in reflecting change in CFTR function, we did define a clinical responder for each parameter as well as a combination of both parameters, based on literature. ${ }^{26}$

Not only the clinical parameters and biomarkers show variability for an individual patient over time, also organoid responses from an individual patient may differ, which indicates that there are still some challenges in the accuracy and/or precision of the organoid measurements. In chapter 7 we show that in a group of homozygous F508del patients the response of a patients' organoid can be different over time. However, we also show that the organoid response of a patient relative to the response of the other patients was maintained. Including reference organoids in the experimental setup enables comparison of an organoid responses over time, within one lab and between different labs over the world. Reference organoids with a known response to CFTR modulators can then not only serve as a quality check but also as a comparison to be able to predict whether or not a patient is responsive to a certain CFTR modulating treatment. 


\section{The use of organoids for personalized medicine in CF}

Currently the decision on the availability of a CFTR modulating drug for a patient is based on the CFTR genotype. This introduces a challenge in finding effective treatments for patients with rare CFTR mutations, since they are often not eligible to be included in a clinical trial and studies on the effect of a therapy are difficult when patient numbers are limited. In vitro drug tests in intestinal organoids might be an option for these patients since organoids are genetically identical to the patient. This not only enables the testing of drugs in patients with very rare CFTR mutations but also creates a setting in which the possible effect of genetic properties of an individual patient, other than the CFTR mutation, can be taken into account when evaluating the effect of a CFTR modulating drug. ${ }^{20}$

In chapter 7 we provide a first example of successful selection of an individual patient with a very rare CFTR mutation for modulator treatment. Treating more patients with a rare CFTR mutation based on intestinal organoid measurements enabled us to generate ROC-curves (chapter 8) for predicting a clinical response of a patient. In this chapter we present the positive predictive value (PPV) and negative predictive value (NPV) of the optimal cut off point for predicting a clinical responder or non-responder, based on the Youden index. The selected value had PPV and NPV of $100 \%$ and $80 \%$ respectively. As a comparison, the results of a study in which change in SCC after two weeks of treatment was used to predict a long term clinical responder (improvement ppFEV $1>5 \%$ after 16 weeks) showed a PPV and NPV of $88 \%$ and $66 \%$ respectively. ${ }^{26}$ Besides higher PPV and NPV values, the organoids have the benefit of in vitro screening without the need to treat the patient. However, the optimal cut off value in the organoids should always be based on the purpose of the screen; different cut-off values can be used to screen for responders and non-responders to drugs. In this thesis we only used the intestinal organoids to select patients for treatment that are not eligible for treatment based on the label of a drug.

When a patients is identified as a possible responder in vitro, a test treatment with the drug can be considered to evaluate clinical efficacy. As mentioned previously, determining the clinical effect of a CFTR modulator in an individual patient is difficult since clinical outcome parameters are influenced by more than CFTR function only. The effect of technical variability as well as the effect of environmental factors can be reduced by performing multiple measurements during a treatment period. Besides improving the accuracy of an individual test it can be an option to combine the results of different clinical parameters in a composite clinical outcome score. This score might be a better reflection of the effect of a CFTR modulator than change in ppFEV , or SCC alone. It is important that the development of such a composite score happens in close cooperation with regulatory authorities such as FDA and EMA, since they decide on the clinical efficacy of a drug. 
The results in chapter 8 on the use of organoids to predict whether a patient will be a clinical responder or non-responder are all based on data of patients with different CFTR mutations. It is known that, for example, not all patients that are homozygous for the F508del mutation show an improvement in $\mathrm{ppFEV}_{1}$ upon treatment with ivacaftor+lumacaftor. More data has to be collected in a group of patients with the same mutation to evaluate if the organoid model can be used for in vitro selection of patients that will probably not have an improvement in $\mathrm{ppFEV}_{1}$ to a drug. At this point we do not have enough data to support exclusion of patients, that are eligible to use a CFTR modulator based on the label of the drug, from treatment.

The results of clinical trials with a so called "triple therapy" show an even bigger improvement in $\mathrm{ppFEV}_{1}$ compared to the effect of ivacaftor+lumacaftor, not only in patients homozygous for the F508del mutations but also for patients with only one F508del mutation (ppFEV $1+10.0 \%$ and $+13.8 \%$ respectively) and has recently been approved by the FDA. ${ }^{27,28}$ When this triple therapy is available for treatment of CF patients all over the world, approximately $90 \%$ of the patients with CF may get access to a CFTR modulating drug. This leaves still $10 \%$ of the CF patients, mostly with two very rare or class I mutations, without any options for CFTR modulatory therapy. In this situation intestinal organoids can be used to evaluate the efficacy of the available CFTR modulators in patients with two very rare mutations. Moreover, the organoid model can be a valuable drug screening tool, not only to find and effective treatment for patients with a class I mutation but also to evaluate the efficacy of newly developed CFTR modulators, also in combination with the currently available drugs.

\section{Organoids for personalized medicine in other diseases}

Organoids are not only a promising tool for personalized medicine in patients with CF, but might also be used for patients with other diseases. The ability to grow organoids from other organs and also tumor cells offers opportunities to use organoids as an in vitro model for many diseases. ${ }^{29,30}$ The organoids offer a platform which can be used to detect and select potentially effective treatments for an individual patient (Fig.2). However, after the identification of a (potentially) effective drug for an individual patient some practical issues arise. As discussed previously, it is often difficult to perform a clinical trial for small groups of patients and even more difficult to evaluate the clinical effect of a treatment in an individual patient. When organoids are used to repurpose an existing treatment or even find a new treatment for an individual patient, it is important that regulatory authorities are consulted in an early stage. In that way more information on how clinical response should be measured and what response is considered to be clinically relevant, can be collected in an early stage of drug development. This will (hopefully) speed up the process of drug approval and reimbursement, also for individual patients. 
Figure 2. Steps from screening of a drug in primary cell cultures (PCC) to drug reimbursement for a patient.

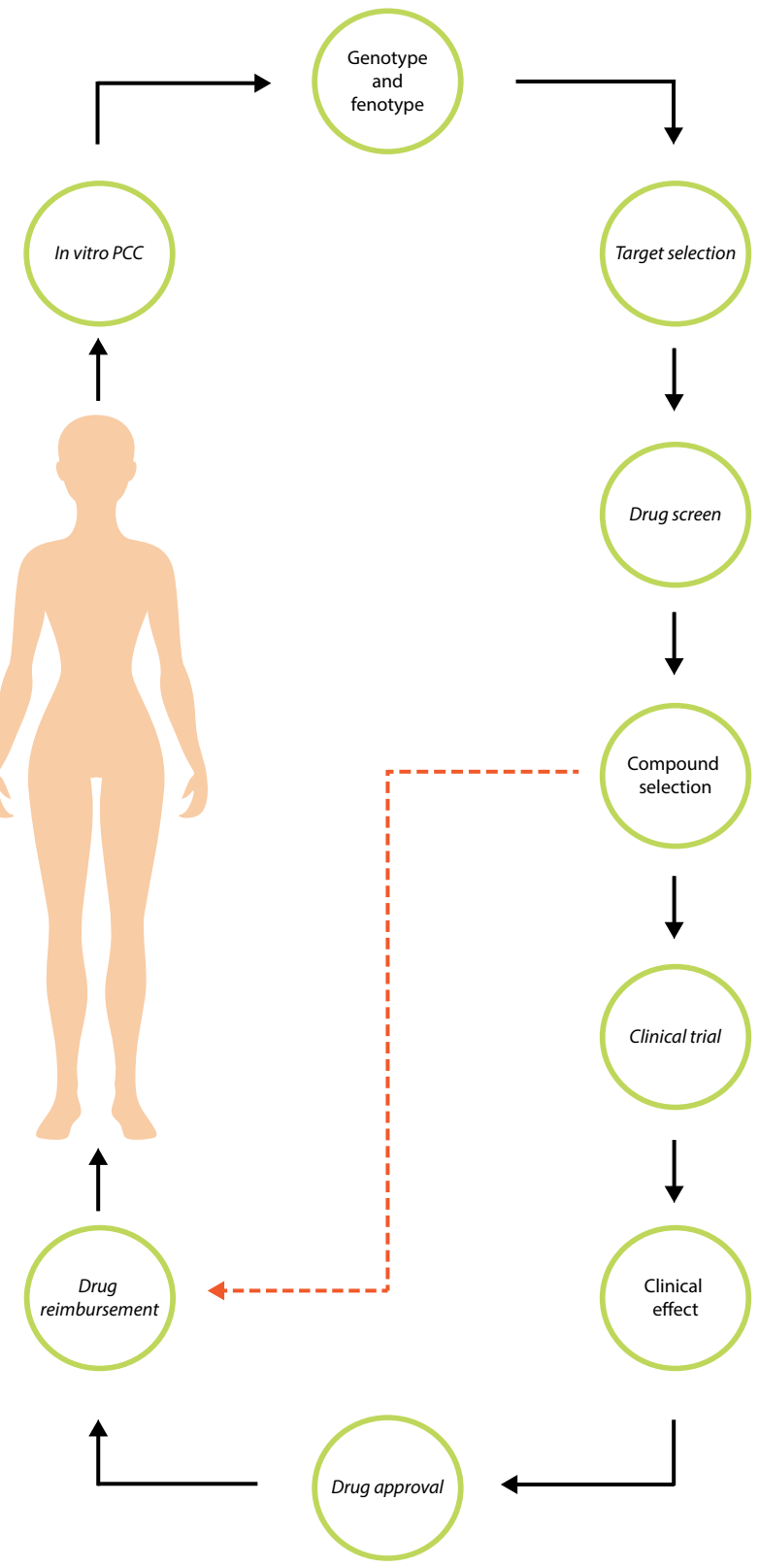

Adapted from freepik.com 


\section{CONCLUDING REMARKS}

The ability to culture organoids from stem cells has led to the generation of patientspecific living biobanks for many diseases. In this thesis we showed the successes and challenges when the in vitro findings in intestinal organoids of CF patients were translated to the in vivo setting. We showed that organoids are a promising tool for identification of new CFTR modulating drugs or testing combinations of known CFTR modulating drugs. Moreover, we also showed that organoids enabled us to successfully select individual CF patients with a rare CFTR mutation for treatment with a modulator drug that induced a clear clinical effect. With this thesis we provided one of the first clinical applications for the use of the organoid model. In the future this model will probably enable more efficient drug screens and the identification of (more) effective treatments, not only in CF but also for other diseases. 


\section{REFERENCES}

1. de Winter-de Groot KM, Janssens HM, van Uum RT, et al. Stratifying infants with cystic fibrosis for disease severity using intestinal organoid swelling as a biomarker of CFTR function. Eur Respir J. 2018;52(3):1702529.

2. Clancy JP, Rowe SM, Accurso FJ, et al. Results of a phase lla study of VX-809, an investigational CFTR corrector compound, in subjects with cystic fibrosis homozygous for the F508del-CFTR mutation. Thorax. 2012;67(1):12-18.

3. Moss RB, Flume PA, Elborn JS, et al. Efficacy and safety of ivacaftor in patients with cystic fibrosis who have an Arg117His-CFTR mutation: a double-blind, randomised controlled trial. Lancet Respir Med. 2015;3(7):524-533.

4. Flume PA, Liou TG, Borowitz DS, et al. Ivacaftor in Subjects With Cystic Fibrosis Who Are Homozygous for the F508del-CFTR Mutation. Chest. 2012;142(3):718-724.

5. De Boeck K, Munck A, Walker $\mathrm{S}$, et al. Efficacy and safety of ivacaftor in patients with cystic fibrosis and a non-G551D gating mutation. J Cyst Fibros. 2014;13(6):674-680.

6. Boyle MP, Bell SC, Konstan MW, et al. A CFTR corrector (lumacaftor) and a CFTR potentiator (ivacaftor) for treatment of patients with cystic fibrosis who have a phe508del CFTR mutation: a phase 2 randomised controlled trial. Lancet Respir Med. 2014;2(7):527-538.

7. Wainwright CE, Elborn JS, Ramsey BW, et al. Lumacaftor-Ivacaftor in patients with cystic fibrosis homozygous for Phe508del CFTR. N Engl J Med. May 2015.

8. Accurso FJ, Rowe SM, Clancy JP, et al. Effect of VX-770 in persons with cystic fibrosis and the G551D-CFTR mutation. N Engl J Med. 2010;363(21):1991-2003.

9. Rowe SM, Daines C, Ringshausen FC, et al. Tezacaftor-Ivacaftor in Residual-Function Heterozygotes with Cystic Fibrosis. N Engl J Med. 2017;377(21):2024-2035.

10. Keating D, Marigowda G, Burr L, et al. VX-445-Tezacaftor-Ivacaftor in patients with cystic fibrosis and one or two Phe508del alleles. N Engl J Med. 2018;379(17):1612-1620.

11. Heltshe SL, Rowe SM, Skalland M, Baines A, Jain M, GOAL Investigators of the Cystic Fibrosis Foundation Therapeutics Development Network. Ivacaftor-treated CF Patients Derive Long-term Benefit Despite No Short-term Clinical Improvement. Am J Respir Crit Care Med. December 2017:rccm.201710-2046LE.

12. Volkova N, Moy K, Evans J, et al. Disease progression in patients with cystic fibrosis treated with ivacaftor: Data from national US and UK registries. J Cyst Fibros. June 2019.

13. Konstan MW, McKone EF, Moss RB, et al. Assessment of safety and efficacy of long-term treatment with combination lumacaftor and ivacaftor therapy in patients with cystic fibrosis homozygous for the F508del-CFTR mutation (PROGRESS): a phase 3, extension study. Lancet Respir Med. December 2016.

14. Ramsey BW, Davies J, McElvaney NG, et al. A CFTR potentiator in patients with cystic fibrosis and the G551D mutation. N Engl J Med. 2011;365(18):1663-1672.

15. Ramsey BW, Nepom GT, Lonial S. Academic, Foundation, and Industry Collaboration in Finding New Therapies. Drazen JM, Harrington DP, McMurray JJV, Ware JH, Woodcock J, eds. N Engl J Med. 2017;376(18):1762-1769.

16. Clancy JP, Cotton CU, Donaldson SH, et al. CFTR modulator theratyping: Current status, gaps and future directions. J Cyst Fibros. 2019;18(1):22-34.

17. Zomer-van Ommen DD, Vijftigschild LAW, Kruisselbrink E, et al. Limited premature termination codon suppression by read-through agents in cystic fibrosis intestinal organoids. J Cyst Fibros. 2016;15(2):158-162.

18. Kerem E, Konstan MW, De Boeck K, et al. Ataluren for the treatment of nonsensemutation cystic fibrosis: a randomised, double-blind, placebo-controlled phase 3 trial. Lancet Respir Med. 2014;2(7):539-547.

19. Zainal Abidin N, Haq IJ, Gardner Al, Brodlie M. Ataluren in cystic fibrosis: development, clinical studies and where are we now? Expert Opin Pharmacother. August 2017:1-9. 
20. Dekkers JF, Wiegerinck $\mathrm{CL}$, de Jonge $\mathrm{HR}$, et al. A functional CFTR assay using primary cystic fibrosis intestinal organoids. Nat Med. 2013;19(7):939-945.

21. Cutting GR. Cystic fibrosis genetics: from molecular understanding to clinical application. Nat Rev Genet. 2014;(November).

22. Collaco JM, Blackman SM, Raraigh KS, et al. Sources of Variation in Sweat Chloride Measurements in Cystic Fibrosis. Am J Respir Crit Care Med. June 2016.

23. Vermeulen F, Le Camus C, Davies JC, Bilton D, Milenković D, De Boeck K. Variability of sweat chloride concentration in subjects with cystic fibrosis and G551D mutations. J Cyst Fibros. 2017;16(1):36-40.

24. Masson A, Schneider-Futschik EK, Baatallah N, et al. Predictive factors for lumacaftor/ ivacaftor clinical response. J Cyst Fibros. December 2018.

25. Fidler MC, Beusmans J, Panorchan P, Van Goor F. Correlation of sweat chloride and percent predicted FEV1 in cystic fibrosis patients treated with ivacaftor. J Cyst Fibros. October 2016.

26. Seliger VI, Rodman D, Van Goor F, Schmelz A, Mueller P. The predictive potential of the sweat chloride test in cystic fibrosis patients with the G551D mutation. J Cyst Fibros. 2013;12(6):706-713.

27. Heijerman HGM, McKone EF, Downey DG, et al. Efficacy and safety of the elexacaftor plus tezacaftor plus ivacaftor combination regimen in people with cystic fibrosis homozygous for the F508del mutation: a double-blind, randomised, phase 3 trial. Lancet. October 2019.

28. Middleton PG, Mall MA, Dřevínek P, et al. Elexacaftor-Tezacaftor-Ivacaftor for Cystic Fibrosis with a Single Phe508del Allele. N Engl J Med. 2019;381(19):1809-1819.

29. Driehuis E, Kolders S, Spelier S, et al. Oral Mucosal Organoids as a Potential Platform for Personalized Cancer Therapy. Cancer Discov. 2019;9(7):852-871.

30. Drost J, Clevers H. Organoids in cancer research. Nat Rev Cancer. 2018;18(7):407-418. 


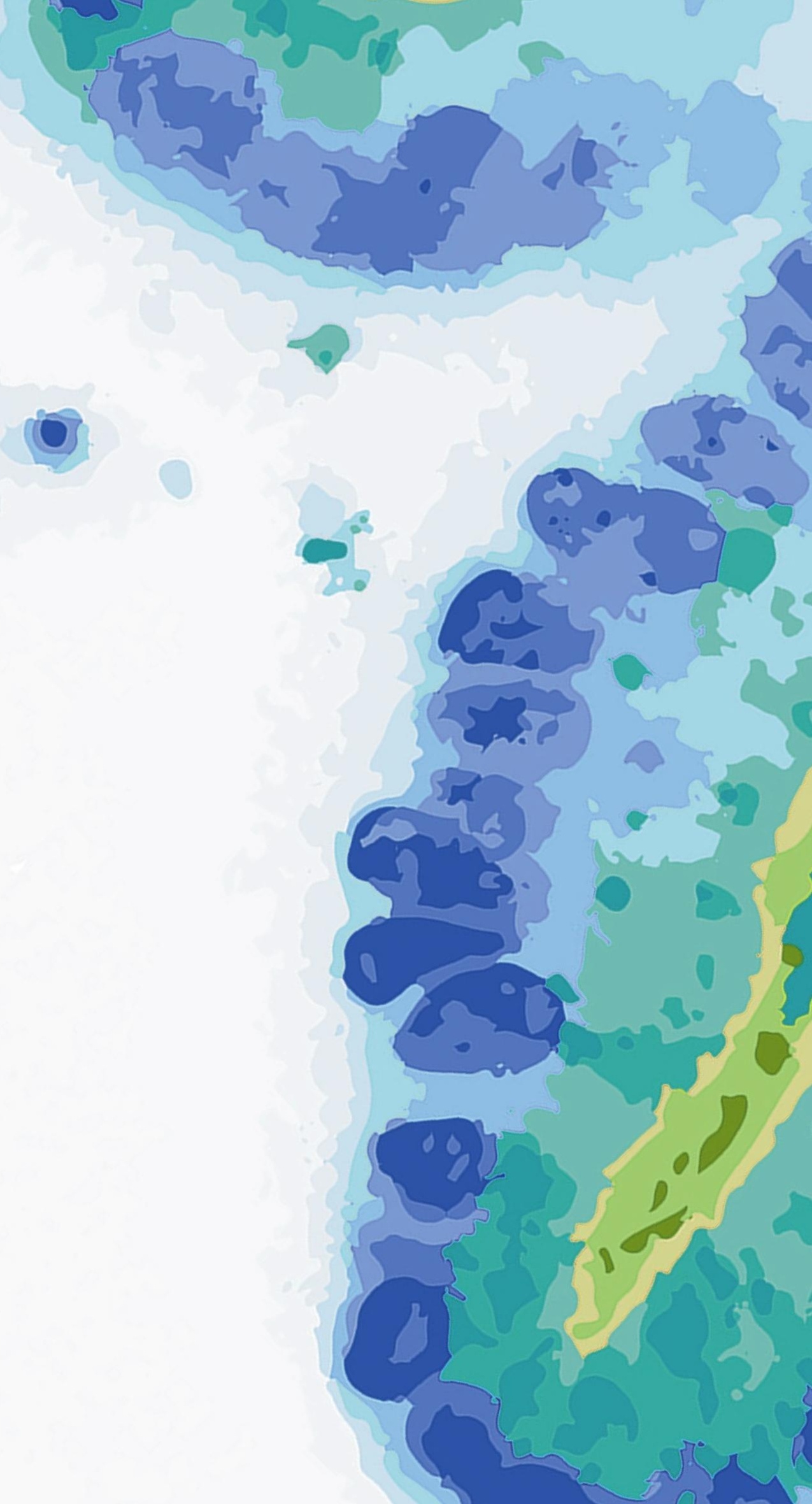




$$
\text { CHAPTER } 10
$$

SUMMARY IN DUTCH NEDERLANDSE SAMENVATTING 
- ledereen is anders. En iedereen is gelijk -

\section{Hoofdstuk 1: Introductie}

Taaislijmziekte of cystic fibrosis (CF) is een erfelijke ziekte die wordt veroorzaakt door mutaties in het gen dat codeert voor het zogeheten cystic fibrosis transmembrane conductance regulator (CFTR) eiwit. lemand heeft CF als hij zowel van zijn vader als van zijn moeder een gen erft met daarop een mutatie in het CFTR-gen. In Nederland is ongeveer 1 op de 30 personen drager van zo'n mutatie en zijn er ongeveer 1500 mensen met CF. Wereldwijd zijn er ongeveer 70.000 mensen die CF hebben.

Het CFTR eiwit zorgt in de cel voor het transport van zouten zoals chloride en bicarbonaat. Hierdoor wordt ook het transport van water geregeld en CFTR is daarmee essentieel voor de waterhuishouding in verschillende organen. Als dit eiwit niet goed werkt kunnen dus problemen ontstaan in het functioneren van meerdere organen zoals bijvoorbeeld de longen, alvleesklier, darmen, lever en het mannelijke voortplantingssysteem. Ondanks dat de levensverwachting van patiënten de laatste jaren is toegenomen is er nog geen curatieve behandeling beschikbaar; de gemiddelde leeftijd van overlijden is momenteel 31 jaar. Dit vroegtijdige overlijden wordt in de meeste gevallen veroorzaakt door longproblemen.

Er zijn meer dan 2000 verschillende mutaties in het CFTR gen beschreven en de combinatie van mutaties die een patiënt heeft (het genotype) is van invloed op de ernst van het ziektebeeld. Zo zijn er mutaties waardoor geen tot nauwelijks functionerend CFTR eiwit wordt geproduceerd; deze mutaties zorgen meestal voor een ernstig ziektebeeld. Er bestaan ook 'mildere' mutaties, waarbij wel een (beperkt) functionerend eiwit wordt gemaakt; deze mutaties zorgen meestal voor een minder ernstig ziektebeeld.

De laatste jaren zijn er veel ontwikkelingen geweest op het gebied van de behandeling van CF. Er zijn nieuwe medicijnen beschikbaar gekomen die niet de symptomen van CF behandelen maar de functie van het eiwit zelf verbeteren. Momenteel zijn er twee soorten van deze zogeheten 'CFTR modulatoren' beschikbaar die bij patiënten met bepaalde mutaties voor een duidelijke verbetering, maar nog geen genezing, van het ziektebeeld kunnen zorgen. Het is dus afhankelijk van het soort mutaties dat een patiënt heeft of hij of zij in aanmerking komt voor een behandeling met deze nieuwe middelen. De uitdaging is momenteel dan ook niet alleen om nog effectievere CFTR modulatoren te ontwikkelen maar ook om modulatoren te vinden en beschikbaar te maken voor iedere CF patiënt. Dit is met name een uitdaging voor patiënten met hele zeldzame CFTR mutaties, onder andere doordat het moeilijk is om in een kleine groep of zelfs bij individuele patiënten onderzoek te doen naar de effectiviteit van deze geneesmiddelen. 
De klinische effectiviteit van CFTR modulatoren wordt in studies vaak gemeten door de conditie van de longen te beoordelen met behulp van de ppFEV1. Dit staat voor'percentage predicted forced expiratory volume in one second' en geeft een beeld van de hoeveelheid lucht die iemand kan uitblazen in de eerste seconde van een geforceerde uitademing. Om de conditie van de longen te beoordelen kunnen ook andere onderzoeken plaatsvinden zoals bijvoorbeeld het maken van scans. Daarnaast wordt vaak de hoeveelheid zout in het zweet bepaald; hoe lager deze hoeveelheid is hoe beter het CFTR eiwit werkt. Andere metingen die regelmatig worden gebruikt om een indruk te krijgen van het functioneren van verschillende organen zijn het meten van: body mass index (BMI, functioneren maagdarm stelsel), elastase in de ontlasting (functioneren alvleesklier) en het afnemen van vragenlijsten (cystic fibrosis questionnaire, CFQ) om een idee te krijgen van de kwaliteit van leven van een patiënt.

Een aantal jaren geleden is een test ontwikkeld waarmee in het laboratorium de functie van het CFTR eiwit van een individuele patiënt kan worden geëvalueerd. Deze test wordt gedaan in een soort bolletjes mini-darm (ook wel organoids genoemd) die gemaakt kunnen worden door eenmalig weefsel af te nemen uit de endeldarm van een patiënt. Door aan de organoids de stof forskoline toe te voegen wordt het CFTR eiwit gestimuleerd. Bij een goede functie van dit eiwit worden zout en water de organoids in getransporteerd waardoor deze opzwellen. We hebben gezien dat bij stimulatie met forskoline de organoids van gezonde personen inderdaad gaan zwellen en ook dat deze zwelling veel minder plaats vindt of zelfs afwezig is in de organoids van patiënten met CF. Deze zwelling is volledig afhankelijk van de functie van het CFTR eiwit en kan dus mogelijk gebruikt worden om de functie van het eiwit te evalueren voor een individuele patiënt (zie ook figuur 1).

In dit proefschrift onderzoeken we in hoeverre de CFTR functie die we meten in de organoids van een patiënt, overeenkomt met de ernst van de ziekte en ook de reactie op CFTR modulatoren van diezelfde patiënt. Dit proefschrift bestaat uit twee delen; in deel I onderzoeken we of we het organoid model kunnen gebruiken om nieuwe CFTR modulatoren te identificeren, in deel II onderzoeken we of we organoids kunnen gebruiken voor 'personalized medicine'. Dit laatste zou met name erg nuttig kunnen zijn voor de behandeling van patiënten met een zeldzame CFTR mutatie. 
Figuur 1. Organoid zwelling in reactie op forskoline als maat voor CFTR functie

a.

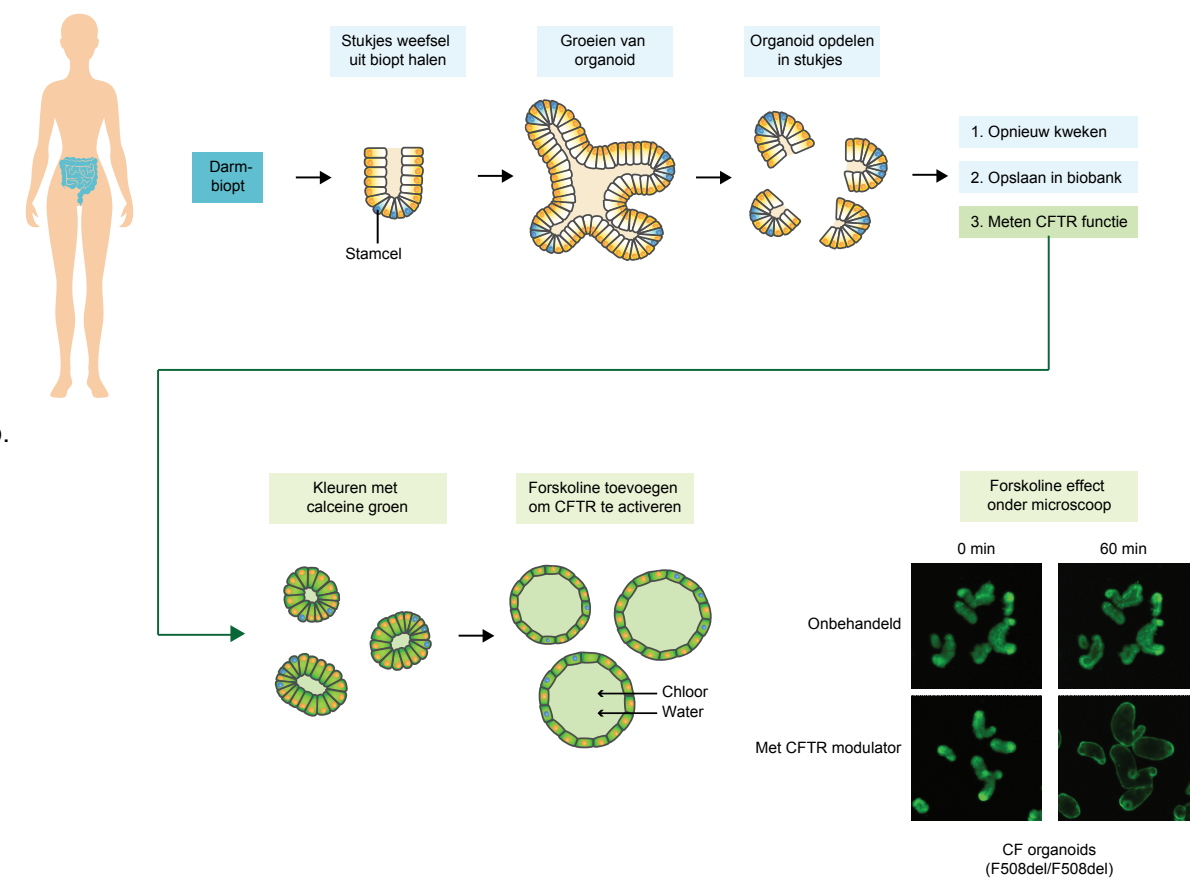

(a) Stamcellen worden uit darmbiopt gehaald en organoids worden gekweekt. Vervolgens kunnen organoids worden opgeslagen of verder gekweekt.

(b) Organoid zwelling in reactie op forskoline met en zonder CFTR modulatoren kan worden onderzocht na labelen van de organoids met calceine groen. 


\section{HET KLINISCH EFFECT VAN VERSCHILLENDE CFTR MODULATOREN}

\section{Hoofdstuk 2: CF-patiënten behandelen met een astmamedicijn}

Bij gezonde mensen is het CFTR eiwit dat aanwezig is aan de oppervlakte van een cel het grootste gedeelte van de tijd gesloten. Door lichaamseigen stoffen wordt vervolgens een signaal gegeven om het eiwit te openen zodat transport plaats kan vinden. In dit hoofdstuk hebben we 61 stoffen geselecteerd die erg lijken op één van deze lichaamseigen stoffen. Deze stoffen hebben we vervolgens toegevoegd aan de organoids van patiënten met een mildere CFTR mutatie waarbij het CFTR eiwit wel aan het oppervlak van de cel zit maar niet optimaal functioneert. We zagen dat een aantal van deze stoffen inderdaad zorgde voor een verbetering van de functie van het aangedane CFTR eiwit. Vervolgens hebben we met een van deze stoffen, het medicijn salbutamol, een kleine klinische studie gedaan waarin we hebben onderzocht wat het effect was wanneer CF-patiënten dit medicijn kortdurend gebruikten. Salbutamol is een bekend medicijn dat zorgt voor het openzetten van de luchtwegen en al jaren wordt gebruikt; bijvoorbeeld in de behandeling van patiënten met astma. Tijdens de studie zagen we een kleine verbetering van het chloortransport gemeten in de neus, wanneer patiënten het medicijn innamen in de vorm van een pil. Ook hebben we voor en na de behandeling bloed afgenomen en onderzocht wat er gebeurde als we dit bloed toevoegden aan de organoids van een patiënt. Ook in de organoids zagen we een kleine verbetering van de CFTR functie in reactie op het bloed. In een vervolgstudie, die niet staat beschreven in dit hoofdstuk, hebben we het effect onderzocht van een langdurige behandeling met salbutamol. Deze studie hebben we echter voortijdig gestopt omdat bleek dat patiënten te veel last hadden van de (bekende) bijwerkingen van salbutamol.

\section{Hoofdstuk 3: Voedingssupplementen als behandeling voor $C F, 1+1=3$ ?}

Bij patiënten met een zogeheten 'gating' mutatie bevindt zich wel een CFTR eiwit aan het celoppervlak maar gaat dit eiwit niet goed open waardoor er minder goed zouten getransporteerd kunnen worden. Ivacaftor is een van de CFTR modulatoren die in de laatste jaren is ontwikkeld en zorgt er bij deze patiënten voor dat het openen van het eiwit verbeterd. Ivacaftor wordt ook wel een 'CFTR-potentiator' genoemd. In eerder onderzoek hebben we gezien dat de organoids van patiënten met een typisch Nederlandse gating mutatie (de S1251N mutatie) niet alleen reageren wanneer we ivacaftor toevoegen maar ook wanneer we de voedingssupplementen curcumine (uit geelwortel) en genistein (bestanddeel van soja) toevoegen. In dit hoofdstuk hebben we onderzocht of niet alleen de organoids maar ook de patiënten zelf reageren op (combinaties van) deze stoffen. We hebben in drie studies het effect onderzocht van een behandeling met curcumine + genistein, ivacaftor en ivacaftor + genistein. De resultaten van deze studies laten alleen 
een duidelijk klinisch effect van ivacaftor zien en we hebben gezien dat behandelingen met de voedingssupplementen niet zorgen voor een duidelijke verbetering in het ziektebeeld van de patiënt. Een mogelijke verklaring zou kunnen zijn dat in de eerdere testen die in de organoids zijn gedaan, veel hogere concentraties zijn gebruikt dan de concentraties die we uiteindelijk terugvinden in het bloed van de patiënten. Dit laat zien dat het belangrijk is om in de organoid testen rekening te houden met de concentratie van het medicijn dat uiteindelijk in het bloed kan worden bereikt.

\section{Hoofdstuk 4: Zorgt ivacaftor voor een verbetering in de galzouten huishouding?}

In dit hoofdstuk hebben we bekeken of het positieve effect van ivacaftor dat we hadden geobserveerd in hoofdstuk 3 ook terug te zien was in parameters die een beeld geven van de productie en opname van galzouten in het maag-darm stelsel. In dit hoofdstuk zagen we op groepsniveau inderdaad een verbetering van deze parameters na een behandeling met ivacaftor maar vonden we voor de individuele patiënt geen duidelijke relatie met verandering in $\mathrm{ppFEV}_{1}$ en zoutgehalte in het zweet.

\section{Hoofdstuk 5: Een bekende CFTR modulator hergebruiken voor een typisch Nederlandse mutatie}

Wereldwijd is de meest voorkomende mutatie de F508del mutatie; ongeveer de helft van de patiënten heeft twee keer deze mutatie. Voor patiënten met twee van deze mutaties is sinds een aantal jaar een CFTR modulerende behandeling beschikbaar. Deze behandeling (Orkambi®) bestaat uit een combinatie van de potentiator ivacaftor (zie ook hoofdstuk 3) en de 'corrector' lumacaftor. Het laatste middel zorgt er voor dat er meer CFTR eiwit aan het celoppervlak komt. In het lab reageren de organoids van patiënten met de typisch Nederlandse A455E mutatie ook goed op Orkambi en in dit hoofdstuk hebben we onderzocht of ook de patiënten zelf een klinische verbetering lieten zien wanneer ze een aantal weken behandeld werden met dit middel. In deze kleine studie zagen we een duidelijke verbetering van het zoutgehalte in het zweet maar geen duidelijke verbetering van de functie van de longen, gemeten met ppFEV1. Omdat er wel duidelijke aanwijzingen waren dat Orkambi een positief effect had in deze patiëntengroep hebben patiënten de mogelijkheid gekregen om na de studie Orkambi te blijven gebruikten. Ook na de studie hebben we metingen gedaan bij deze patiënten en gezien dat ze na een halfjaar niet alleen een duidelijke verbetering hadden van het zoutgehalte in het zweet maar ook een duidelijke verbetering van de functie van de longen. 


\title{
II. ORGANOIDS GEBRUIKEN OM VOORSPELLINGEN TE DOEN VOOR EEN INDIVIDUELE PATIENT
}

\author{
Hoofdstuk 6: Ziekte ernst bepalen in het laboratorium
}

Doorgaans hebben patiënten met een 'mildere' CFTR mutatie meer restfunctie van het CFTR eiwit, wat meestal resulteert in een milder klinisch beloop van de ziekte. Dit in tegenstelling tot patiënten met 'zware' mutatie die vaak weinig restfunctie hebben en ook een slechter klinisch beloopt. Eerder onderzoek heeft al laten zien dat we met organoids de milde mutaties van de zware mutaties kunnen onderscheiden. Nou is het zo dat er ook bij patiënten met dezelfde mutatie verschillen bestaan van ziekte-ernst. In dit hoofdstuk hebben we onderzocht of er ook binnen een groep patiënten met een zelfde mutatie (F508del, zie ook hoofdstuk 5) een relatie bestaat tussen de restfunctie gemeten in de organoids en het klinisch beeld van de patiënt. De resultaten van dit hoofdstuk laten zien dat er inderdaad een relatie is tussen de zwelling van een organoid van een patiënt en zijn of haar conditie van de longen en voedingstoestand.

\section{Hoofdstuk 7: Effectiviteit van medicijnen in organoids en patiënten - komt het overeen?}

Zoals al eerder genoemd, zijn er veel verschillende CFTR mutaties die kunnen zorgen voor verschillen in ziekte-ernst en ook voor verschillende reacties op CFTR modulatoren. In dit hoofdstuk hebben we om te beginnen bekeken in hoeverre de restfunctie van het CFTR kanaal, gemeten in de organoids van de patiënt, overeenkomt met de ernst van de CFTR mutatie. Hierbij vonden we een duidelijke relatie tussen de metingen in de organoids en metingen van CFTR functie in de patiënt zelf. Vervolgens hebben we bekeken of de reacties van de organoids op verschillende CFTR modulatoren van patiënten met verschillende mutaties overeenkwamen met de effecten die gevonden waren in de klinische studies waarin patiënten met een zelfde mutatie met hetzelfde middel behandeld werden. Ook hierbij zagen we een duidelijke relatie tussen het effect dat we hadden gemeten in de organoids en het klinisch effect dat de patiënten in de studies hadden. Met behulp van deze kennis hebben we vervolgens voor het eerst twee patiënten met een zeer zeldzame CFTR mutatie succesvol kunnen selecteren voor een behandeling met een CFTR modulator. Dit was een belangrijke eerste stap in het gebruik van het organoid model voor 'personalized medicine' in CF patiënten. 


\section{Hoofdstuk 8: Organoids gebruiken om individuele patiënten te behandelen}

Dat we in hoofdstuk 7 twee patiënten met een zeer zeldzame CFTR mutatie succesvol hebben kunnen selecteren voor een behandeling met een CFTR modulator was erg hoopgevend! In dit hoofdstuk hebben we verder onderzocht of, en zo ja hoe, we de organoids van een individuele patiënt kunnen gebruiken om voor die patiënt te voorspellen of hij of zij goed zal reageren op een behandeling met een CFTR modulator. We hebben hiervoor bekeken hoe vaak we de klinische respons van een patiënt goed konden voorspellen bij verschillende afkappunten in de respons van de organoids van een patiënt. Met andere woorden: hoe veel moeten de organoids van een patiënt zwellen in reactie op een medicijn voordat we denken dat die patiënt zelf ook echt een positief effect heeft van het medicijn. Met het afkappunt waarmee we het 'beste' een voorspelling konden doen konden we $100 \%$ van de patiënten zonder positief klinisch effect en $80 \%$ van de patiënten met positief klinisch effect goed voorspellen.

\section{CONCLUSIE}

De mogelijkheid om organoids te kweken vanuit cellen uit het menselijk lichaam biedt heel veel mogelijkheden voor onderzoek. Niet alleen voor patiënten met CF maar ook voor onderzoek naar andere ziektes zoals bijvoorbeeld onderzoek in de oncologie. In dit proefschrift hebben we niet alleen laten zien dat er een duidelijke relatie bestaat tussen de resultaten van organoid metingen en het ziektebeeld van dezelfde patiënt, maar ook dat we organoids kunnen gebruiken om effectieve behandelingen te vinden voor (groepen) patiënten die in het huidige systeem van effectiviteitsstudies in grote 'randomized controlled trials' vaak buiten de boot vallen. Het onderzoek dat staat beschreven in dit proefschrift heeft er voor een heel aantal patiënten met CF toe geleid dat ze behandeld konden worden met een levens veranderend medicijn. In de toekomst zal het organoid model ongetwijfeld gebruikt kunnen worden om dit voor nog meer patiënten te realiseren, niet alleen voor patiënten met CF maar ook voor patiënten met andere ziektes. 


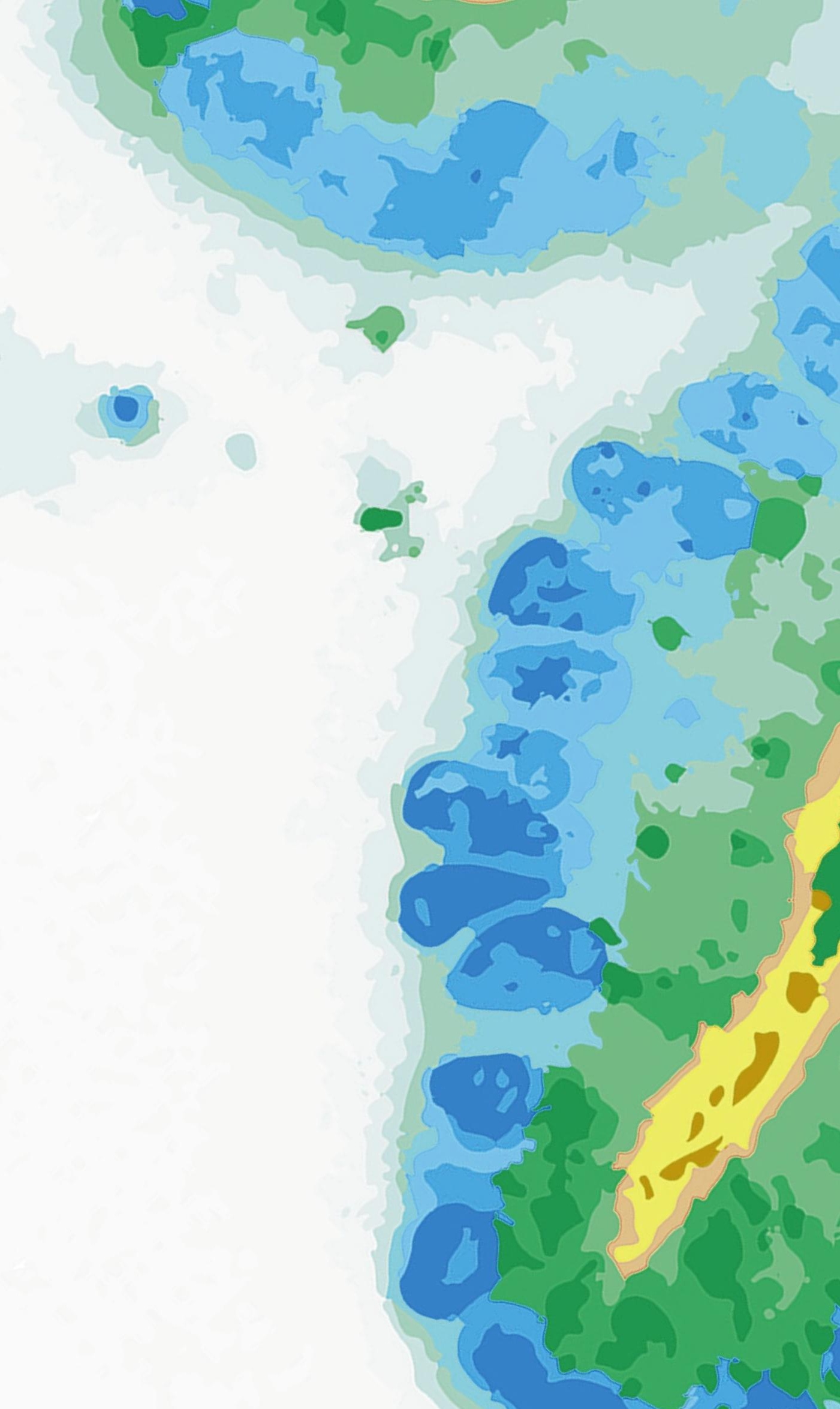




$$
\text { CHAPTER } 11
$$

ADDENDA 


\section{ABBREVIATIONS}

\begin{tabular}{|c|c|}
\hline$A B C$ & ATP-binding cassette \\
\hline $\mathrm{AE}$ & adverse event \\
\hline Amil & amiloride \\
\hline ASBT & apical sodium bile acid transporter \\
\hline ATP & adenosine triphosphate \\
\hline$A \cup C$ & area under the curve \\
\hline BA & bile acid \\
\hline BAD & bile acid diarrhea \\
\hline BMI & body mass index \\
\hline C4 & A-hydroxy-4-cholesten-3-one \\
\hline CAMP & cyclic adenosine monophosphate \\
\hline CF & cystic fibrosis \\
\hline CFBE & cystic fibrosis bronchial epithelial \\
\hline CFF & cystic fibrosis foundation \\
\hline CFTR & cystic fibrosis transmembrane conductance regulator \\
\hline CFQ-r & cystic fibrosis questionnaire - revised \\
\hline $\mathrm{Cl}$ & confidence interval \\
\hline $\mathrm{Cl}$ & chloride \\
\hline CT & computed tomography \\
\hline CYP7A1 & cholesterol 7a-hydroxylase \\
\hline DMEM & dulbecco's modified eagle medium \\
\hline DMSO & dimethylsulphoxide \\
\hline DNA & deoxyribonucleic acid \\
\hline EDTA & ethylenediaminetetraacetic \\
\hline ELISA & enzyme-linked immuno sorbent assay \\
\hline EMA & European medicines agency \\
\hline $\mathrm{ENaC}$ & epithelial sodium channel \\
\hline FE & fecal elastase \\
\hline FEV1 & forced expiratory volume in one second \\
\hline FGF-19 & fibroblast growth factor 19 \\
\hline FGFR4 & fibroblast growth factor receptor 4 \\
\hline FDA & American food and drug administration \\
\hline FIS & forskolin-induced swelling \\
\hline FVC & forced vital capacity \\
\hline FXR & farnesoid $X$ receptor \\
\hline Gl & gastrointestinal \\
\hline GPCR & G-protein coupled receptor \\
\hline HBE & human bronchial epithelial \\
\hline HEPES & hydroxyethyl piperazineethanesulfonic \\
\hline HTS & high throughput screen \\
\hline
\end{tabular}


$\mathrm{HZ}$

ICH GCP

ICM

IQR

Isc

Iso

IVA

$\mathrm{LCI}$

LC-MS

LS

LUM

mEGF

MMRM

mRNA

NBD's

NCFS

NPD

NPV

PCC

$P D$

$\mathrm{PI}$

ppFEV1

PPV

Raw

ROC

SCC

SD

SE

SEM

SIBO

SLA

SOP

UDCA

VX-445

$V X-661$

$V X-770$

VX-809

WT

YFP homozygous

international council for harmonisation good clinical practice intestinal current measurement

interquartile range

short circuit current

isoprenaline

ivacaftor

lung clearance index

liquid chromatography-mass spectrometry

least squares

lumacaftor

murine epidermal growth factor

mixed-effects model for repeated measures

messenger ribonucleic acid

nucleotide binding domains

Nederlandse cystic fibrosis stichting

nasal potential difference

negative predictive value

primary cell cultures

potential difference

pancreatic insufficiency

percentage of predicted forced expiratory volume in 1 second positive predictive value

airway resistance

receiver operating characteristic

sweat chloride concentration

standard deviation

standard error

standard error of the mean

small intestinal bacterial overgrowth

steady-state lumen area

standard operating procedure

ursodeoxycholic acid

elexacaftor

tezacaftor

ivacaftor

lumacaftor

wild-type

yellow fluorescent protein 


\section{CONTRIBUTING AUTHORS}

\section{Paul Audhya}

Vertex Pharmaceuticals Incorporated

\section{Hubertus G.M. Arets}

Department of Pediatric Pulmonology

University Medical Center Utrecht

\section{Marleen Bakker}

Department of Pulmonology

Erasmus University Medical Center

\section{Jeffrey M. Beekman}

Department of Pediatric Pulmonology and Regenerative Medicine Center Utrecht University Medical Center Utrecht

\section{Frank A. J. A. Bodewes}

Pediatric Gastroenterology and Hepatology

University Medical Center Groningen

\section{Inez Bronsveld}

Department of Pulmonology

University Medical Center Utrecht

\section{Hans C. Clevers}

Hubrecht Institute for Developmental Biology and Stem Cell Research

University Medical Center Utrecht

\section{Johanna F. Dekkers}

Hubrecht Institute for Developmental Biology and Stem Cell Research University Medical Center Utrecht

\section{Jasper S. Dijkema}

Department of Pediatric Pulmonology

University Medical Center Utrecht

\section{Marcela Doktorova}

Section of Molecular Metabolism and Nutrition and Pediatric Gastroenterology and Hepatology

University Medical Center Groningen 


\section{Sjoerd G. Elias}

Department of Epidemiology, Julius Center for Health Sciences and Primary Care University Medical Center Utrecht

\section{Cornelis K. van der Ent}

Department of Pediatric Pulmonology

University Medical Center Utrecht

\section{Johanna C. Escher}

Department of Pediatric Gastroenterology

Erasmus University Medical Centre

\section{Margot Geerdink}

Department of Pediatric Pulmonology

University Medical Center Utrecht

\section{Eduard A. van de Graaf}

Department of Pulmonology

University Medical Center Utrecht

\section{John W. Hanrahan}

CF Translational Research Centre, Department of Physiology

McGill University Montréal

\section{Harry G.M. Heijerman}

Department of Pulmonology

University Medical Center Utrecht

\section{Chantal E. Hensen}

Department of Pediatric Pulmonology

University Medical Center Utrecht

\section{Roderick H.J. Houwen}

Department of Pediatric Gastroenterology

University Medical Center Utrecht

\section{Hettie M. Janssens}

Department of Pediatrics, div. Respiratory Medicine and Allergology

Erasmus University Medical Center 


\section{Hugo R. de Jonge}

Department of Gastroenterology and Hepatology

Erasmus University Medical Center

\section{Johan W. Jonker}

Section of Molecular Metabolism and Nutrition

University Medical Center Groningen

\section{Marleen Kemper}

Department of Pharmacy

Amsterdam University Medical Centers

\section{Nils Kinnman}

Vertex Pharmaceuticals Incorporated

\section{Gerard H. Koppelman}

Department of Pediatric Pulmonology and Pediatric Allergology and GRIAC Research Institute

University Medical Center Groningen

\section{Evelien Kruisselbrink}

Department of Pediatric Pulmonology and Regenerative Medicine Center Utrecht University Medical Center Utrecht

\section{Christof J. Majoor}

Department of Respiratory Medicine

Amsterdam University Medical Centers

\section{Rozemarijn E.P. Marck-van der Wilt}

Department of Pediatric Pulmonology

University Medical Center Utrecht

\section{Elizabeth Matthes}

CF Translational Research Centre, Department of Physiology

McGill University Montréal

\section{Renske van der Meer}

Department of Pulmonology

Haga Teaching Hospital 


\section{Sabine Michel}

Department of Pediatric Pulmonology

University Medical Center Utrecht

\section{Peter van Mourik}

Department of Pediatric Pulmonology

University Medical Center Utrecht

\section{Edward E.S. Nieuwenhuis}

Department of Pediatric Gastroenterology

University Medical Center Utrecht

\section{Ivo P. van de Peppel}

Section of Molecular Metabolism and Nutrition and Pediatric Gastroenterology and Hepatology

University Medical Center Groningen

\section{Yolanda B. de Rijke}

Department of Clinical Chemistry

Erasmus University Medical Center

\section{Jolt Roukema}

Department of Pediatric Pulmonology

Radboud University Medical Center

\section{Sylvia W.F. Suen}

Department of Pediatric Pulmonology Regenerative Medicine Center Utrecht University Medical Center Utrecht

\section{Henkjan J. Verkade}

Section of Molecular Metabolism and Nutrition and Pediatric Gastroenterology and Hepatology

University Medical Center Groningen

\section{Maaike, M. Vanderschuren}

Department of Pediatric Pulmonology

University Medical Center Utrecht

\section{Lodewijk A.W. Vijftigschild}

Department of Pediatric Pulmonology and Laboratory of Translational Immunology University Medical Center Utrecht 


\section{Frank P. Vleggaar}

Department of Gastroenterology \& Hepatology

University Medical Center Utrecht

\section{Annelotte M. Vonk}

Department of Pediatric Pulmonology Regenerative Medicine Center Utrecht University Medical Center Utrecht

\section{Robert G.J. Vries}

Hubrecht Organoid Technology (HUB)

\section{Karin M. de Winter - de Groot}

Department of Pediatric Pulmonology

University Medical Center Utrecht

\section{Zheng (Jason) Yuan}

Vertex Pharmaceuticals Incorporated

\section{Domenique D. Zomer-van Ommen}

Department of Pediatric Pulmonology and Laboratory of Translational Immunology University Medical Center Utrecht 


\section{ACKNOWLEDGEMENTS - DANKWOORD}

Zoals gebruikelijk eindigt ook dit proefschrift met een dankwoord, en terecht! Dit proefschrift was er niet geweest zonder de hulp en steun van heel veel mensen. Graag wil ik een aantal mensen in het bijzonder bedanken.

Kinderen en volwassenen met $C F$, jullie bereidheid om steeds maar weer mee te doen aan de verschillende studies vond ik bewonderenswaardig. Bedankt daarvoor!

Kors, als promotor heb jij mij altijd alle vrijheid en vertrouwen gegeven om mijzelf te kunnen ontwikkelen. Ik heb veel van je geleerd de afgelopen jaren. Ik bewonder de rust waarmee jij situaties benadert en het overzicht dat je weet te bewaren. Daarnaast waardeer ik je droge humor en positieve instelling; successen moet je vieren! Ik kijk met heel veel plezier terug op mijn promotietraject: heel erg bedankt voor deze leuke en leerzame tijd.

Jeff, ook jou mag ik nu officieel mijn promotor noemen. Je hebt enorm veel parate kennis en bent ontzettend gedreven. Dat al je werk nu bekroond is met je Professor titel lijkt mij dan ook niet meer dan terecht. Je bent niet alleen een hele harde werker, maar ook een uniek persoon die zijn mening niet onder stoelen of banken steekt en regelmatig zonder censuur zegt wat er in je hoofd omgaat. Ik waardeer je eerlijkheid en heb de afgelopen jaren niet alleen veel met je kunnen lachen, maar ook altijd heel fijn met je samengewerkt. Ontzettend bedankt!

Leden van de promotiecommissie, Prof. dr. H.G.M. Heijerman, Prof. dr. W.L. van der Pol, Prof. dr. G.H. Koppelman, Prof. dr. R.H.J. Houwen en Prof. dr. N.M. Wulffraat, hartelijk dank voor het beoordelen van mijn manuscript en jullie bereidheid om zitting te nemen in de promotiecommissie.

Coauteurs, heel erg bedankt voor jullie waardevolle bijdrage aan dit proefschrift.

Afdeling kinderlongziekten en -allergologie, een fantastische afdeling om als eerste werkplek te hebben! Het is mooi om te zien hoe jullie als team betrokken zijn bij elkaar en bij de zorg voor de patiënten. Ik wil jullie niet alleen bedanken voor de hulp en interesse op onderzoeksgebied, maar ook voor de vele gezellige taartmomenten, borrels, kerstontbijten en ga zo maar door.

Rolien, Valesca, Hetty en Joyce, heel erg bedankt voor jullie hulp de afgelopen jaren bij alle studievisites en uitleg over de, soms ingewikkelde, longfunctiemetingen.

Cora en Marit, bedankt dat jullie deur eigenlijk altijd open stond, zowel letterlijk als figuurlijk. Door af en toe een dagje bij jullie op de kamer te zitten realiseerde ik me maar weer al te goed waar we al dit onderzoek voor doen; de patiënten. Ik bewonder jullie betrokkenheid bij alle CF-patiënten. Jullie zijn een mooi duo! 
Het researchteam, Sabine, Margot, Ilse, Stephan, Hannah en Emma, heel erg bedankt voor al jullie hulp bij de klinische studies die we samen hebben gedaan. Zonder jullie hulp was dit proefschrift nog lang niet klaar geweest.

Kinderlongartsen, Bert en Karin, bedankt voor jullie inzet de afgelopen jaren bij het includeren van patiënten en het samenwerken aan het onderzoek.

Myriam, vaak klopte ik met één probleem bij je aan en loste je er twee voor me op. Dankjewel voor al je hulp, betrokkenheid, warmte en het feit dat je altijd voor mij klaar stond!

Team Beekman, in de jaren van mijn promotie heb ik jullie zien groeien van een team van 5 mensen naar een groep van meer dan 20 man sterk. Jullie zijn een mooi team! Evelien en Annelot, van het begin af aan hebben we samengewerkt. Jullie zorgen samen voor structuur, heel veel data, leuke inhoudelijke discussies en veel gezelligheid. Bedankt voor al die dingen. Florijn, Domenique, Lodewijk, Eyleen en Marne, met jullie heb ik samengewerkt aan de vertaling van het werk op het lab naar de kliniek. Ik vond het heel leuk om af en toe een kijkje te nemen bij jullie op het lab en dat ik zelfs af en toe mocht pipeteren (bijna altijd met de pipet goed om). Ik wil jullie bedanken voor alle uitleg en natuurlijk gezelligheid.

Team Beekman, ik wil jullie allemaal bedanken dat jullie mij altijd hebben betrokken bij jullie team. Ik voelde me altijd heel welkom als ik een dagje bij jullie in het Hubrecht kwam werken. Tot slot wil ik jullie ook heel erg bedanken voor jullie geduld bij het uitleggen van de lab technieken tijdens jullie lab meetings. Naast dat ik heel gezellig vond om met jullie te werken, heb ik ook heel veel van jullie geleerd!

Een speciaal plekje in dit dankwoord voor Hugo. Ik bewonder al je kennis en passie voor onderzoek. Je was niet alleen projectleider van het "curcumin-genistein project", maar gaf ook waardevolle input voor een heel aantal andere hoofdstukken van dit proefschrift. Heel erg bedankt voor de fijne samenwerking in de afgelopen jaren.

Mede-promovendi, het was een feest om iedere dag met een fijne club collega artsonderzoekers om me heen te kunnen werken. In de afgelopen jaren heb ik met heel wat verschillende mensen op de kamer gezeten. Kim, Jacobien, Nicole, Francine, Nienke, Astrid, Sabine P., Maartje, Peter, Hannah, Maaike, Danya en Sabine vd L., allemaal heel erg bedankt voor de welkome koffiepauzes waarin we alle (onderzoeks-) lief en leed even de revue konden laten passeren. Het leven kan er soms heel anders uit zien na een lekkere cappuccino!

Wetenschapsstudenten, Chantal, Roos, Jasper, Maaike en Joshena, bedankt voor jullie inzet en hulp bij het uitvoeren, structureren en analyseren van alle studies. Ik hoop dat ik iets van mijn enthousiasme voor het onderzoek aan jullie heb kunnen overbrengen. Op mijn beurt heb ik in ieder geval veel van jullie geleerd. 
Skate4air-collega's, Een hele waardevolle herinnering uit mijn onderzoekstijd is ons avontuur op de Weissenzee. Marian, Erik, Wytze, Peter, Nienke, Margot en Bert, ook al was het soms afzien, ik heb heel veel plezier beleefd aan onze trainingen, het samen ophalen van sponsorgeld en natuurlijk de week in Oostenrijk, bedankt daarvoor!

CF-centra in Nederland, bedankt voor de fijne samenwerking in de afgelopen jaren. Jullie bijdrage aan alle klinische studies die in dit proefschrift staan is ontzettend waardevol geweest!

Nederlandse Cystic Fibrosis Stichting, bedankt voor de fijne samenwerking en de financiële middelen om dit onderzoek te kunnen uitvoeren.

Paranimfen, de afgelopen jaren voelde het figuurlijk al zo en ik vind het dan ook heel fijn dat jullie op deze dag letterlijk aan mijn zijde willen staan! Sabine, vanaf de eerste week dat ik op de afdeling kwam werken zijn we samen aan de slag gegaan om heel wat klinische trials tot een goed einde te brengen. Ik heb niet alleen altijd heel fijn met je samengewerkt, maar ik heb ook veel goede herinneringen aan onze koffiedates, kerstpakketten-inpak-ochtenden, leuke congressen en Amerika tripjes met bijbehorende shopsessies daaromheen. Heel erg bedankt daarvoor! Nienke, vanaf de eerste dag zaten we bij elkaar op de kamer en hebben we samen het promotieavontuur beleefd. Ik denk dat we elkaar de afgelopen jaren mooi hebben aangevuld in ons onderzoekswerk ("als jij mijn tekst controleert, maak ik dat figuur voor jou"). Het is fijn om te weten dat je altijd tijd maakt als ik behoefte heb aan een luisterend oor en advies, dankjewel daarvoor!

Vrienden, natuurlijk is er ook een leven en heel veel plezier naast werk. Allemaal heel erg bedankt voor jullie vriendschap. Clubgenootjes, Sanne, Lisanne, Emma, Jennifer, Janneke, Anne Marie, Caroline, Marlou en Joelle. We zijn allemaal heel verschillend, maar zijn toch al meer dan 10 jaar een mooie vriendinnengroep. Ik denk met veel plezier terug aan alle avonturen die we hebben beleefd en kijk uit naar de vele mooie momenten die ongetwijfeld nog zullen komen. Karlijn, met heel veel plezier denk ik terug aan de jaren dat we huisgenootjes waren op de Doornstraat. Al is het niet meer op een zitzak voor de deur van ons studentenhuis, ik ben blij dat we nog regelmatig samen wijntjes drinken in de zon. Julia, we zijn al vriendinnen sinds de middelbare school en ik vind het heel fijn dat we allebei nog steeds tijd maken om af en toe bij te kletsen. Dat voelt altijd meteen als vanouds! Emilie, Angela en Christie, sinds jaar 1 van onze studie geneeskunde zijn we vriendinnen en samen genieten we al jaren van gezellige etentjes, borrels, sportsessies en heerlijke vakanties. Bedankt voor jullie onvoorwaardelijke steun en vriendschap!

(Schoon)familie, Wat een mooie groep van (stief- en/of schoon-) ouders, broer(tjes) en zusjes (doorhalen waar nodig) heb ik om me heen! De trotse kring van 8 opa's en oma's toen Lize op haar eerste verjaardag het kaarsje op de taart probeerde uit te blazen is daar denk ik een heel mooi voorbeeld van. Dank jullie wel dat jullie er altijd voor mij zijn. 
Lieve (stief)zusjes en broertje: Ruth, Loek, Milou en Renee. We woonden de afgelopen jaren in alle hoeken van het land (of daarbuiten), maar vinden elkaar toch altijd weer ergens terug. Bedankt voor de gezellige etentjes, skivakanties, flitsbezoekjes, borrels, het oppassen en natuurlijk al jullie gezelligheid! Natuurlijk ook veel dank voor mijn ouders. Papa, regelmatig kom je "even een kopje koffie drinken". Dat je daarvoor twee uur in de auto zit maakt je niks uit en dit is denk ik kenmerkend voor jouw warme persoonlijkheid. Mama, bedankt voor al jouw onvoorwaardelijke steun en betrokkenheid. Het is fijn om te weten dat ik altijd bij je terecht kan.

Lize, lief kleintje. Wat ben ik blij dat jij erbij bent gekomen in deze periode. Dankjewel voor alle welkome afleiding, het doorslapen in de nacht en jouw intense vrolijkheid!

Lieve Rik, samen kunnen we alles, dat bewijst dit proefschrift ook maar weer. Dankjewel dat je mij tijdens deze hele promotie een fijne thuisbasis, veel steun en altijd vertrouwen hebt gegeven. Ik ben trots op ons als gezinnetje en heb veel zin in de tijd die komen gaat! 


\section{LIST OF PUBLICATIONS}

\section{This thesis}

Berkers $G$, van der Meer R, van Mourik P, Vonk AM, Kruisselbrink E, Suen SWF, Heijerman HGM, Majoor CJ, Koppelman GH, Roukema J, Janssens HM, de Rijke YB, Kemper EM, Beekman JM, van der Ent CK, de Jonge HR. Clinical effects of the three CFTR potentiator treatments curcumin, genistein and ivacaftor in patients with the CFTR-S1251N gating mutation. J. Cyst. Fibros. June 2020.

de Winter - de Groot KM, Berkers G, Marck - van der Wilt REP, et al. Forskolin-induced swelling of intestinal organoids correlates with disease severity in adults with cystic fibrosis and homozygous F508del mutations. J Cyst Fibros. November 2019.

Berkers G, van Mourik P, Vonk AM, et al. Rectal organoids enable personalized treatment of cystic fibrosis. Cell Rep. February 2019.

van de Peppel IP, Doktorova M, Berkers G, et al. IVACAFTOR restores FGF19 regulated bile acid homeostasis in cystic fibrosis patients with an S1251N or a G551D gating mutation. J Cyst Fibros. September 2018.

Vijftigschild LAW, Berkers G, Dekkers JF, et al. $\beta 2$-Adrenergic receptor agonists activate CFTR in intestinal organoids and subjects with cystic fibrosis. Eur Respir J. July 2016.

Dekkers JF, Berkers G, Kruisselbrink $E$, et al. Characterizing responses to CFTRmodulating drugs using rectal organoids derived from subjects with cystic fibrosis. Sci Transl Med. June 2016.

\section{Other publications}

Burghard M, Berkers G, Ghijsen S, Hollander-Kraaijeveld FM, de Winter-de Groot KM, van der Ent CK, Heijerman HGM, Takken T and Hulzebos HJ. Long-term Effects of Ivacaftor on Nonpulmonary Outcomes in Individuals With Cystic Fibrosis, Heterozygous for a S1251N Mutation. Pediatr Pulmonol. June 2020.

Geurts MH, de Poel E, Amatngalim GD, Oka R, Meijers FM, Kruisselbrink E, van Mourik P, Berkers G, de Winter-de Groot KM, Michel S, Muilwijk D, Aalbers BL, Mullenders J, Boj SF, Suen SWF, Brunsveld JE, Janssens HM, Mall MA, Graeber SY, van Boxtel R, van der Ent CK, Beekman JM, and Clevers H. CRISPR-Based Adenine Editors Correct Nonsense Mutations in a Cystic Fibrosis Organoid Biobank. Cell Stem Cell. February 2020.

de Winter-de Groot KM, Janssens HM, van Uum RT, Dekkers JF, Berkers G, Vonk AM, Kruisselbrink E, Oppelaar H, Vries R, Clevers H, Houwen RHJ, Escher JC, Elias SG, de Jonge 
HR, de Rijke YB, Tiddens HAWM, van der Ent CK, and Beekman JM. Stratifying infants with cystic fibrosis for disease severity using intestinal organoid swelling as a biomarker of CFTR function. Eur Respir J. September 2018.

Dekkers JF, Van Mourik P, Vonk AM, Kruisselbrink E, Berkers G, de Winter-de Groot KM, Janssens HM, Bronsveld I, van der Ent CK, de Jonge HR, and Beekman JM. Potentiator synergy in rectal organoids carrying S1251N, G551D, or F508del CFTR mutations. J Cyst Fibros. May 2016.

Berkers G, Biesaart MCIH, Leeuwenburgh-Pronk WG. Disciplinary verdicts in cases of child abuse; Lessons for paediatricians. Ned Tijdschr Geneeskd. March 2015. 


\section{CURRICULUM VITAE}

Gitte Berkers was born on December 12th 1988 in Venray, the Netherlands. She grew up in Nijmegen where se graduated from secondary school at the Stedelijk Gymnasium Nijmegen in 2006. In the same year she moved to Utrecht to start her medical training at the Faculty of Medicine of Utrecht University. During medical school she did an internship Obstetrics and Gynaecologie at the Muhimbili University Of Health and Allied Sciences in Tanzania. Her enthusiasm for research started in 2012 when she did an elective research internship at

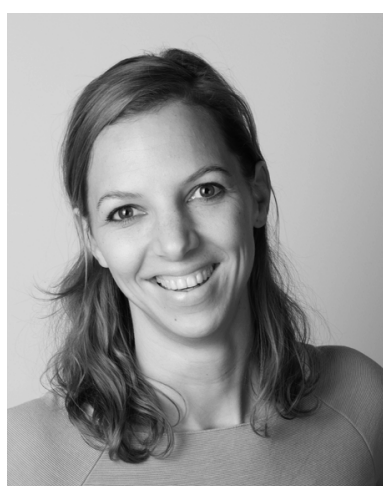
the "Regionaal Tuchtcollege Amsterdam". After that she performed a research internship at the department of pediatric pulmonology and allergology in the Wilhelmina Children's Hospital Utrecht.

In 2013 she graduated from Medical School and in 2014 she started her PhD project at the department of pediatric pulmonology and allergology in the Wilhelmina Children's Hospital Utrecht, focusing on personalized treatment of cystic fibrosis patients, under supervision of Prof. Dr. C.K. van der Ent and Prof. Dr. J.M. Beekman. In 2019 she obtained her Master of Science degree in Clinical Epidemiology at the University of Utrecht. In march 2020 she started working as a physician at Me-doc. Via this organization she works at the "St. Pieters en Bloklands Gasthuis"; a health care center in Amersfoort.

Gitte lives in Utrecht, together with Rik and their two year old daughter Lize. 



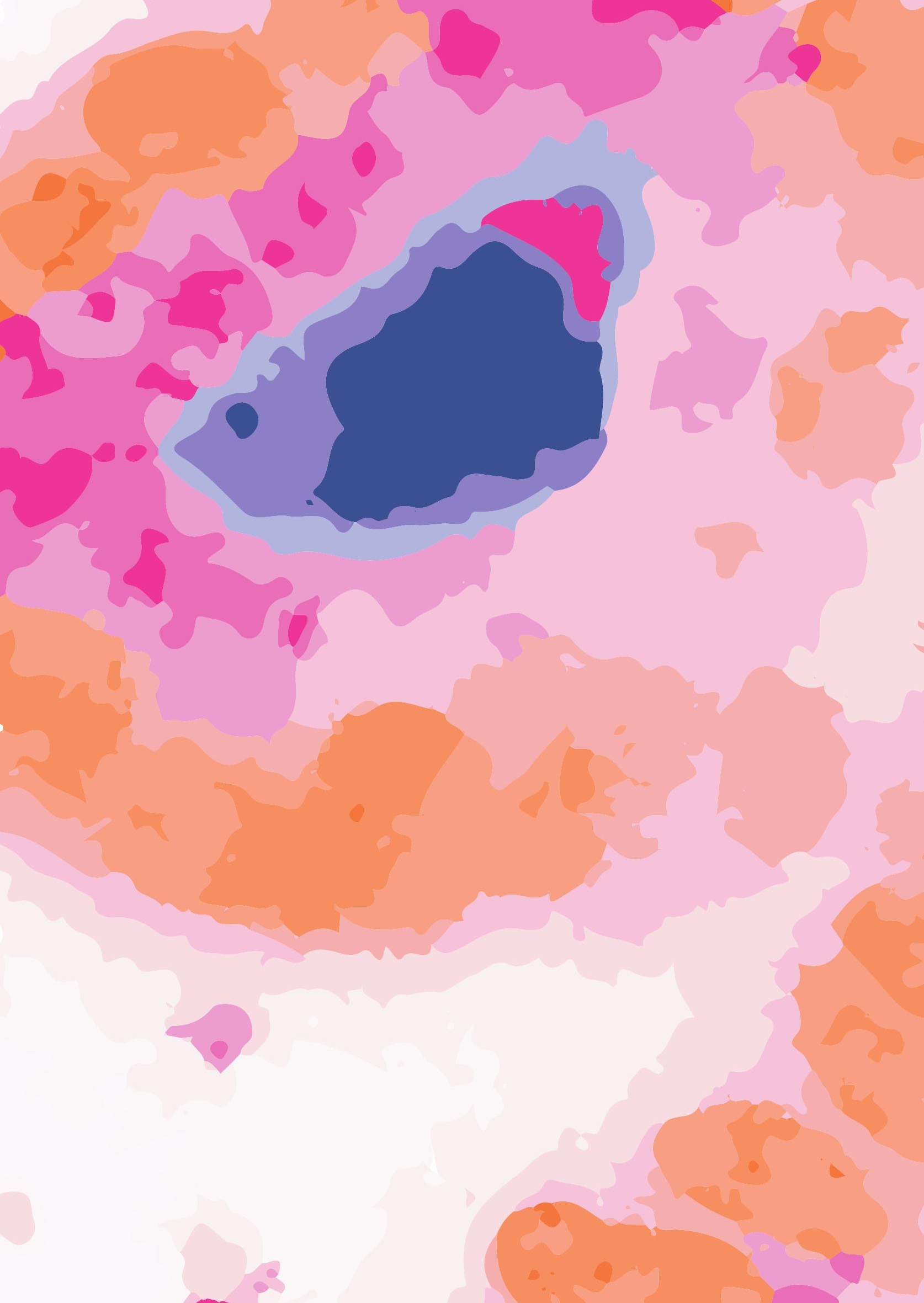

